\title{
Analysis of the RAB family of GTPases in $C$. elegans and their role in regulating neuronal membrane trafficking
}

\author{
PhD Thesis \\ in partial fulfilment of the requirements \\ for the degree "Doctor of Philosophy (PhD)" \\ in the Neuroscience Program \\ at the Georg August University Göttingen, \\ Faculty of Biology
}

Submitted by

Nikhil Sasidharan

Born in

Attingal, India 
I hereby declare that the PhD thesis entitled, "Analysis of the RAB family of GTPases in $C$. elegans and their role in regulating neuronal membrane trafficking", was written independently and with no other sources and aids than quoted.

Göttingen, $14^{\text {th }}$ March, 2011

Nikhil Sasidharan

Thesis Committee Members:

Dr. Stefan Eimer

European Neuroscience

Institute

Prof. Dr. Nils Brose

Max Planck Institute for

Experimental Medicine

Prof. Dr. Walter Stühmer

Max Planck Institute for Experimental Medicine 


\section{TABLE OF CONTENTS}

Table of Contents

List of Figures

i

List of Tables

iv

Abbreviations

vi

Acknowledgements

vii

ix

I. Abstract

Chapter 1: Systematic analysis of the RAB GTPase family in C. elegans 3

II. Introduction 4

2.1 Intracellular transport is regulated by GTPases 4

2.2 Monomeric GTPases $\quad 6$

2.3 Principles of Rab GTPase structure $\quad 8$

2.3.1 The Rab GTPase fold 8

2.3.2 Structural insights on Rab-GAP interactions 10

2.3.3 Structural insights on Rab-GEF interactions $\quad 10$

2.3.4 Structural insights on Rab-Effector interactions 11

2.3.5 Structural insights on Rab localization $\quad 12$

2.4 Principles of Rab GTPase function $\quad 13$

2.4.1 Rabs define sub-cellular compartments 13

2.4.2 Role of Rab GTPases in vesicle budding and uncoating $\quad 15$

2.4.3 Role of Rab GTPases in vesicle motility 16

2.4.4 Role of Rab GTPases in vesicle tethering 17

2.4.5 Role of Rab GTPases in vesicle fusion 19

2.5 Integration of Rab function $\quad 19$

2.5.1 Rabs form microdomains $\quad 19$

2.5.2 Rab conversion cascades $\quad 21$

2.5.3 Rab exclusion cascades 22

2.6 Cell-type specific functions of Rabs $\quad 22$

2.7 Redundancy in Rab function $\quad 23$

$\begin{array}{ll}\text { III. Aims } & 24\end{array}$

$\begin{array}{ll}\text { IV. Results } & 27\end{array}$

4.1 Expression pattern analysis of C. elegans RABs 27

4.2 Sub-cellular localization analysis of C. elegans RABs in neurons $\quad 30$

4.3 Functional analysis of $r a b$ mutants $\quad 32$

4.3.1 Movement analysis $\quad 34$

4.3.2 Defecation motor program analysis $\quad 36$

$\begin{array}{ll}\text { 4.3.3 Egg-laying analysis } & 37\end{array}$

4.4 Pharmacological analysis of synaptic transmission 38

$\begin{array}{ll}\text { 4.4.1 Aldicarb sensitivity } & 38\end{array}$

$\begin{array}{ll}\text { 4.4.2 Levamisole sensitivity } & 40\end{array}$ 
4.5 Analysis of ciliated sensory neurons in rab mutants 41

4.5.1 DiI staining of amphids and phasmids 41

4.5.2 Analysis of amphid neuron function 43

4.5.3 Synthetic RNAi analysis of $r a b$ mutants $\quad 44$

$\begin{array}{ll}\text { V. Discussion } & 46\end{array}$

5.1 The C. elegans genome encodes 28 differentially expressed RABs 46

5.2 Multiple RABs localize to the Golgi in neurons 48

5.3 RAB function in the C. elegans nervous system 48

5.3.1 Role of RABs in regulating simple behaviors 48

5.3.2 Role of RABs in regulating synaptic transmission $\quad 50$

5.3.3 Role of RABs in regulating chemosensation 51

5.4 C. elegans RABs function redundantly together $\quad 52$

Chapter 2: Regulation of dense core vesicle secretion by RAB GTPases $\quad 54$ in C. elegans

$\begin{array}{ll}\text { VI. Introduction } & 55\end{array}$

6.1 The SV exocytosis machinery $\quad 55$

6.2 The DCV exocytosis machinery $\quad 60$

6.3 DCV biogenesis and maturation $\quad 60$

6.4 DCVs function to regulate synaptic transmission $\quad 61$

6.5 Studying DCV function in C. elegans 62

$\begin{array}{ll}\text { VII. Aims } & 65\end{array}$

$\begin{array}{ll}\text { VIII. Results } & 67\end{array}$

8.1 RAB-5 and RAB-10 are novel RABs essential for DCV release $\quad 67$

8.2 DCV trafficking defects are autonomous to the nervous system 69

8.2.1 Development of a neuron-specific RNAi system 69

8.2.2 RAB-5 and RAB-10 cell-specifically regulate DCV release 70

8.3 Identification of $\mathrm{RAB}$ regulators involved in DCV release $\quad 71$

8.4 RAB-5, TBC-2, RAB-10 and TBC-4 also affect secretion of INS-22 73

8.5 Endocytosis in coelomocytes is not affected 74

8.6 Synaptic vesicle release is not affected 74

8.6.1 Analysis of SV markers: SNB-1 and RAB-3 74

8.6.2 EM analysis of SV distributions in synapses 76

8.6.3 EM analysis of neuron morphology 78

8.6.4 Electrophysiological analysis of synaptic transmission $\quad 78$

$\begin{array}{ll}8.7 \text { TBC-4 is a potential GAP for RAB-10 } & 78\end{array}$

8.8 Rabaptin- 5 bridges RAB-5 and RAB-10 function 82

8.9 EHBP-1 also regulates DCV release and interacts with RAB-10 84

8.10 Colocalization analysis of novel factors involved in DCV release $\quad 84$

$\begin{array}{lr}\text { IX. Discussion } & 88\end{array}$

9.1 RAB-5 and RAB-10 are novel regulators of DCV secretion 88 
9.2 A RAB-5 / RAB-10 exclusion cascade 90

9.3 Localization of the RAB-5/RAB-10 exclusion cascade: cell body 93 or synapse?

$\begin{array}{ll}\text { X. Materials and Methods } & 96\end{array}$

10.1 Molecular Cloning $\quad 96$

10.2 C. elegans genetics 97

10.2.1 Strains 97

10.2.2 Crosses 97

10.2.3 Generation of transgenic lines 98

$\begin{array}{ll}10.3 \text { C. elegans behavioral assays } & 99\end{array}$

$\begin{array}{ll}\text { 10.3.1 Movement analysis } & 99\end{array}$

10.3.2 Defecation motor program analysis $\quad 99$

10.3.3 Egg-laying analysis $\quad 99$

10.3.4 Pharmacological assays $\quad 99$

$\begin{array}{ll}10.4 \text { DiI staining } & 100\end{array}$

$\begin{array}{ll}10.5 \text { Chemosensation assays } & 100\end{array}$

10.6 RNAi by feeding $\quad 100$

10.7 Confocal microscopy and image analysis $\quad 101$

$\begin{array}{ll}10.8 \text { TR-BSA endocytosis assay } & 101\end{array}$

10.9 High-pressure freeze electron microscopy 102

$\begin{array}{ll}10.10 \text { Electrophysiology } & 102\end{array}$

$\begin{array}{ll}10.11 \text { Yeast-2-hybrid } & 102\end{array}$

10.12 Protein expression and purification 103

$\begin{array}{lr}\text { XI. References } & 104\end{array}$

$\begin{array}{lr}\text { XII. Appendix } & 122\end{array}$

12.1 Constructs and bacteria/yeast strains $\quad 122$

12.2 C. elegans strains $\quad 132$

$\begin{array}{ll}12.3 \text { Injection mixes for extrachromosomal arrays } & 136\end{array}$

$\begin{array}{ll}12.4 \text { Deletion alleles } & 138\end{array}$

$\begin{array}{ll}12.5 \text { DNA sequences of primers } & 138\end{array}$

$\begin{array}{ll}12.6 \text { Media } & 144\end{array}$

$\begin{array}{ll}12.7 \text { Buffers and solutions } & 145\end{array}$

12.8 Gene structures of $t b c-4$ and rabn-5 deletion alleles $\quad 147$

12.9 Multiple sequence alignment of TBC-4 148

$\begin{array}{lr}\text { Curriculum Vitae } & 149\end{array}$

$\begin{array}{ll}\text { List of publications } & 151\end{array}$ 


\section{LIST OF FigURES}

Figure II.1

Figure II.2

Figure II.3

Figure II.4

Figure II.5

Figure II.6

Figure II.7

Figure II.8

Figure II.9

Figure II.10

Figure II.11

Figure II.12

Figure II.13

Figure III.1

Figure III.2

Figure IV.1

Figure IV.2

Figure IV.3

Figure IV.4

Figure IV.5

Figure IV.6

Figure IV.7

Figure IV.8

Figure IV.9

Figure IV.10

Figure IV.11

Figure IV.12

Figure IV.13
The major sub-cellular compartments of eukaryotic cells

Vesicular transport between organelles occurs in 4 four distinct steps

Regulation of vesicular transport by GTPases

7

Detailed structure of Rab GTPases 9

Structure of a Rab-GAP complex in its transition state $\quad 10$

Variations in Rab-Effector complexes $\quad 12$

Role of REP, RabGGTase and RabGDI on Rab localization 13

Rabs localize to a variety of compartments 14

$\begin{array}{ll}\text { Rabs interact with motor proteins } & 16\end{array}$

$\begin{array}{ll}\text { Model for tethering by Golgins } & 18\end{array}$

Rabs localize to microdomains 20

Model of a Rab conversion cascade $\quad 21$

Model of Rab conversion and exclusion cascade 22

Comparison of the H. sapiens and C. elegans Rab families 25

Schematic representation of project aims 26

Strategy for expression pattern analysis $\quad 27$

Expression patterns of C. elegans rabs 28

Strategy for sub-cellular localization analysis $\quad 30$

Sub-cellular localization of C. elegans RABs in 31 nervous system

Gene structures of all rabs 33

The neuro-muscular system of C. elegans 34

Movement analysis of rab mutants $\quad 35$

The defecation motor program $\quad 36$

Defecation analysis of rab mutants 37

Egg-laying analysis of $r a b$ mutants $\quad 38$

Analysis of synaptic transmission through 39 responses to aldicarb

Analysis of synaptic transmission through $\quad 40$ responses to levamisole

Anatomic depiction of C. elegans chemosensory neurons 41 
$\begin{array}{lll}\text { Figure IV.14 DiI staining of } r a b \text { mutants } & 42\end{array}$

Figure IV.15 Responses of $r a b$ mutants to copper and isoamyl alcohol 43

Figure IV.16 Synthetic RNAi screen with rab mutants 45

Figure VI.1 The synaptic vesicle cycle and its machinery 56

Figure VI.2 Domain structure of Munc13-1 57

$\begin{array}{lll}\text { Figure VI.3 Domain structure of CAPS-1 } & 59\end{array}$

$\begin{array}{lll}\text { Figure VI.4 } & \text { DCV maturation } & 61\end{array}$

$\begin{array}{lll}\text { Figure VI.5 Regulation of SV release by GPCRs } & 62\end{array}$

Figure VI.6 Studying DCV function in C. elegans 63

Figure VII.1 A rab-5 mutant suppresses DCV defects in rab-2 mutants 66

$\begin{array}{lll}\text { Figure VIII.1 NLP-21-YFP analysis of all rabs } & 68\end{array}$

$\begin{array}{lll}\text { Figure VIII.2 Validation of neuron-specific RNAi } & 69\end{array}$

Figure VIII.3 Neuron-specific knockdown of $r a b-5$ and $r a b-10 \quad 71$

Figure VIII.4 TBC-2 and TBC-4 are novel regulators of DCV secretion 72

Figure VIII.5 $\quad r a b-5(Q 78 L), t b c-2, r a b-10$ and $t b c-4$ mutants also have 73 defects in INS-22 secretion

Figure VIII.6 Assessment of coelomocyte function through 75

$\begin{array}{lll}\text { Figure VIII.7 Analysis of SV markers } & 76\end{array}$

$\begin{array}{lll}\text { Figure VIII.8 } & \text { HPF-EM analysis of synapses } & 77\end{array}$

$\begin{array}{lll}\text { Figure VIII.9 } & \text { HPF-EM analysis of neuron morphology }\end{array}$

$\begin{array}{lll}\text { Figure VIII.10 Electrophysiological analysis of synaptic function } & 80\end{array}$

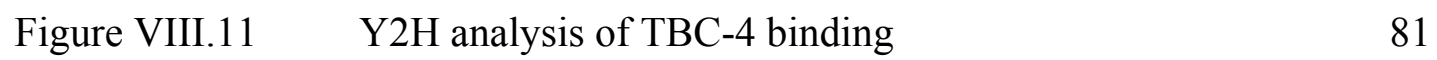

Figure VIII.12 Y2H analysis of TBC-4 interaction with RABN-5 82

Figure VIII.13 NLP-21-YFP analysis of RABN-5 and EEA-1 83

Figure VIII.14 Y2H and NLP-21-YFP analysis of a RAB-10 effector $\quad 85$

Figure VIII.15 Fine mapping of the sub-cellular localization of 86

Figure VIII.16 Sub-cellular localization analysis of RAB-5 (Q78L), $\quad 87$

Figure IX.1 Model of a RAB-5 / RAB-10 exclusion cascade 92

$\begin{array}{lll}\text { Figure XII.1 Gene structures of } t b c-4 \text { and } r a b n-5 & 147\end{array}$

$\begin{array}{lll}\text { Figure XII.2 } & \text { Multiple sequence alignment of TBC-4 }\end{array}$ 


\section{LIST OF TABLES}

Table IV.1 Tabulated representation of expression pattern data

Table IV.2

Tabulated representation of sub-cellular localization data

32

Table XII.1

List of all bacterial/yeast strains and constructs

Table XII.2 used in study

Table XII.3

List of all strains used in this study

Table XII. 4

List of all injection mixes used to generate transgenic lines

136

List of all deletion alleles with respective worm

138

Table XII.5 PCR primers used in this study

138 


\section{AbBreviations}

aBoc

$\mathrm{ACh}$

AChE

Arl

Anterior body contraction

CaM

CAPS

$\mathrm{CC}$

$\mathrm{CCV}$

$\mathrm{CH}$

COG

COPI

COPII

DAG

Acetylcholine

Acetylcholinesterase

Arf-like

DBD

DCV

DENN

DGK

DNC

ECM

EEA-1

EGF

Emc

EPSC

ER

ERGIC

FGFR

FIP2

GABA

GAP

GARP

GDI

GDP

GEF

GFP

GGTase

GLUT4

GPCR

GST

GSV

GTP

HOPS

Calmodulin

$\mathrm{Ca}^{2+}$-dependent activator protein for secretion

Coiled coil

Clathrin coated vesicle

Calponin homology

Conserved oligomeric complex

Coat protein complex I

Coat protein complex II

Diacylglycerol

Dynactin binding domain

Dense core vesicle

Differentially expressed normal versus neoplastic

Diacylglycerol kinase

Dorsal nerve cord

Extra-cellular milieu

Early endosomal antigen-1

Epidermal growth factor

Enteric motor contraction

HPF-EM

HSN

iDCV

$\mathrm{IP}_{3}$

IPTG

M6P

Excitatory postsynaptic current

Endoplasmic reticulum

ER-Golgi intermediate compartments

Fibroblast growth factor receptor

Rab11 family interacting protein-2

$\gamma$-Aminobutyric acid

GTPase activating protein

Golgi associated retrograde protein complex

GDP dissociation inhibitor

Guanosine diphosphate

Guanine nucleotide exchange factors

Green fluorescent protein

Geranylgeranyl transferase

Glucose transporter-4

G-protein coupled receptor

Glutathione-S-transferase

Glucose transporter-4 containing secretory vesicle

Guanosine triphosphate

M6PR

Homotypic vacuole protein sorting complex

MANNS

High pressure freeze-electron microscopy

Hermaphrodite specific neuron

Immature dense core vesicle

Inositol $(1,4,5)$ trisphosphate

isopropyl $\beta$-D-thiogalactopyranoside

Mannose-6-phosphate

Mannose-6-phosphate receptors

Mannosidase 


$\begin{array}{ll}\text { MDCK } & \text { Madin-Darby Canine Kidney } \\ \text { mDCV } & \text { Mature dense core vesicle } \\ \text { nAChR } & \text { Nicotinic ACh receptor } \\ \text { NGM } & \text { Nematode growth medium } \\ \text { NMJ } & \text { Neuro-muscular junction } \\ \text { NSF } & \text { N-ethylmaleimide-sensitive factor } \\ \text { OCRL } & \text { Oculocerebrorenal syndrome of Lowe } \\ \text { ORP1L } & \text { Oxysterol-binding protein related protein 1L } \\ \text { pBoc } & \text { Posterior body contraction } \\ \text { PH } & \text { Pleckstrin homology } \\ \text { PIP } & \text { Phosphoinositol (4,5) bisphosphate } \\ \text { PLC } & \text { Phospholipase C } \\ \text { PM } & \text { Plasma membrane } \\ \text { Rab } & \text { Ras genes from rat brain } \\ \text { RABN-5 } & \text { Rabaptin-5 } \\ \text { REP } & \text { Rab escort protein } \\ \text { RFP } & \text { Red fluorescent protein } \\ \text { RILP } & \text { Rab interacting lysosomal protein } \\ \text { SNAREs } & \text { Soluble N-ethylmaleimide-sensitive factor attachment protein receptors } \\ \text { SV } & \text { Synaptic vesicle } \\ \text { Syt-1 } & \text { synaptotagmin-1 } \\ \text { TBC } & \text { Tre-2/Cdc16/Bub2 } \\ \text { TGN } & \text { Trans-Golgi network } \\ \text { TIP47 } & \text { Tail-interacting protein of 47 kDa } \\ \text { TR-BSA } & \text { Texas-red conjugated to bovine serum albumin } \\ \text { TRAPP } & \text { Transport protein particle complex } \\ \text { VNC } & \text { Ventral nerve cord } \\ \text { VSV-G } & \text { Vesicular stomatitis virus glycoprotein } \\ \text { WT } & \text { Wild type } \\ \text { Y2H } & \text { Yeast-2-hybrid } \\ \text { YFP } & \text { Yellow fluorescent protein } \\ \end{array}$




\section{ACKNOWLEDGEMENTS}

The last three and a half years have been an intensive, wonderful experience and there have been a number of individuals whom I must acknowledge for the part they played.

First I would like to thank Dr. Stefan Eimer for giving me the opportunity to work in his lab, for sharing his knowledge and his never-ending enthusiasm for science. Moreover, I am thankful for his supervision and introducing me to the field of C. elegans neurobiology.

I extend my gratitude to Prof. Dr. Nils Brose and Prof. Dr. Walter Stühmer for their interest, advices and suggestions during the thesis committee meetings. Furthermore, I am also grateful to Prof. Dr. Mikael Simons for agreeing to be an additional member of my thesis committee, for his excitement and advices during the course of the project

The Göttingen Graduate School for Neurosciences and Molecular Biosciences (GGNB) and Neurosciences coordination offices have helped me immensely through administrative work and also through assisting in the overall organization of the $\mathrm{PhD}$. For this I am thankful to Prof. Dr. Michael Hörner, Dr. Steffen Burkhardt, Sandra Drube and Kirsten Poehlker. Being a recipient of the GGNB excellence fellowship, I further acknowledge the GGNB graduate school for financial support during the course of my PhD.

I am thankful to Dr. Jan Hegermann for electron microscopy experiments. Special thanks to Prof. Dr. Alexander Gottschalk for electrophysiology experiments and Prof. Dr. Barth Grant for discussions and sharing of reagents. I am also grateful to Dr. Aymelt Itzen for a lot of advice and suggestions in handling proteins. I must acknowledge Jonas Barth, Alejandro Mendoza and Aniket Ghosh for help during their internships and lab rotation projects. I am especially thankful to all the members of my lab for assisting me with experiments and pushing the Rab project forward. 
Additionally I cannot forget to recognize the excellent technical assistance provided by Dr. Sabine Koenig and Katrin Schwarze.

I have never regretted any of my decisions because they have always led me into the company of good people. To Dr. Sumakovic, I am simply lucky to have had shared an office and a lab with you. Cloning, imaging, injections, coffee, cloning, imaging, injections, coffee, coffee and a little bit of SDS gel on the side. I loved it all. To Mandy, thank you for being exactly the person that was missing in our office. I am thankful also for all the bowling, and the table tennis games (I will win 1 day!!!) and more importantly for teaching an Indian what it means to be German. I am also thankful to the other members of our lab: Christian Olendrowitz, Nora Wender, Ling Luo and Maike Kittelmann for creating a friendly atmosphere and for their support.

I am thankful to the other members of my batch: Ramya Nair, Raunak Sinha and Dr. Chou-hua Huang for their friendship. I also acknowledge, Dr. Shahaf Peleg for his friendship, making me laugh and for the many schnitzel sessions.

I am particularly thankful to my flat mate Dr. Peer Hoopmann for agreeing to live with me, which is not an easy decision to make. I enjoyed it all. There is always going to be some food waiting for him on Saturdays.

I am also thankful to my buddies Sagar Bora, Eugen Nacu, Tadas Jucikas and Andrew Jack Knott for their friendship, and for their constant willingness to help wash clean my brain.

And last I would like to thank my bebek, for making every day more beautiful. 
Dedicated to my parents and my brother Rinosh, for their constant love and support 


\section{AbStract}

Rab GTPases are master regulators of intracellular trafficking. They are involved in every aspect of membrane transport from vesicle budding to vesicle fusion. Their functions are regulated by guanine nucleotide exchange factors (GEFs) and GTPase activating proteins (GAPs). The mammalian genome encodes over 60 different Rab proteins. Each of these Rabs localizes to specific compartments. The actions of multiple Rabs are coordinated through Rab cascades where a single Rab recruits the machinery to activate or inactivate a secondary Rab. Considering the large Rab family size, very little is known about the majority of its members and how they work together.

As opposed to the human system, the C. elegans RAB family encodes 28 members with normally just one isoform per Rab member. The smaller Rab family size, together with the genetic tractability of $C$. elegans allowed for a broad-scale analysis of each RAB protein. For this analysis it was decided to initially determine the expression pattern and sub-cellular localization of all C. elegans RABs. Expression pattern analyses revealed that $C$. elegans rabs are differentially expressed with a pattern of preference for the nervous system. Additionally, the sub-cellular localization analysis of the Rabs showed that they localize to specific sub-cellular compartments with many displaying partial staining to the Golgi apparatus. The availability of mutants for $90 \%$ of the rabs in $C$. elegans further provided the opportunity to elucidate novel RAB functions. Since most RABs were neuronally expressed, all rab mutants were tested for changes in a set of nervous system mediated behaviors: movement, defecation motor program, and egg laying. Analysis revealed that several RABs are important regulators of these behaviors. Additionally all $r a b$ mutants were also tested for defects in synaptic transmission through sensitivity to aldicarb. Several new RABs were identified to modulate aldicarb sensitivity. Mutant analysis showed that although several rab mutants displayed 
phenotypes of nervous system dysfunction, the majority of animals were healthy. This suggested that many RABs function redundantly together in C. elegans. To elucidate which RABs cooperate together, a synthetic RNAi screen was conducted where each rab mutant was co-depleted with the remaining 27 Rabs. Co-depletion of multiple rabs has provided novel insights into the higher order RAB network.

In the second chapter of this study, we aimed to identify novel RABs involved in dense core vesicle (DCV) signaling. Interestingly $r a b-5$ and $r a b-10$ mutants showed defects in DCV secretion. Additionally, two TBC (Tre-2/Cdc16/Bub2) domain-containing molecules, TBC-2 and TBC-4, were identified to function as potential GAPs for RAB-5 and RAB-10 respectively. Lastly we have identified an interaction between a RAB-5 effector, Rabaptin-5 (RABN-5), and TBC-4, which provides a link between RAB-5 and RAB-10 function. Taken together, these results suggest the existence of a novel Rab exclusion cascade in the regulation of DCV release, where active RAB-5 recruits TBC-4 for the local inactivation of RAB-10. 


\section{CHAPTER 1:}

\section{SYSTEMATIC ANALYSIS OF THE RAB GTPASE FAMILY IN C. ELEGANS}




\section{INTRODUCTION}

\subsection{Intracellular transport is regulated by GTPases}

Eukaryotic cells are defined by their compartmentalized endo-membrane system. As opposed to prokaryotes, these cells evolved the ability to organize biomolecules to form sub-cellular organelles, such as the endoplasmic reticulum (ER), the Golgi apparatus, endosomes and lysosomes (Fig. II.1). Each organelle is membrane-bound, spatially segregated and specialized to carry out a particular function. The ER serves as a center to synthesize membrane proteins, lysosomal hydrolases and proteins destined for secretion (Alberts et al., 2008). The Golgi acts as a sub-cellular hub by receiving proteins from one compartment, processing them and sorting them to other compartments.

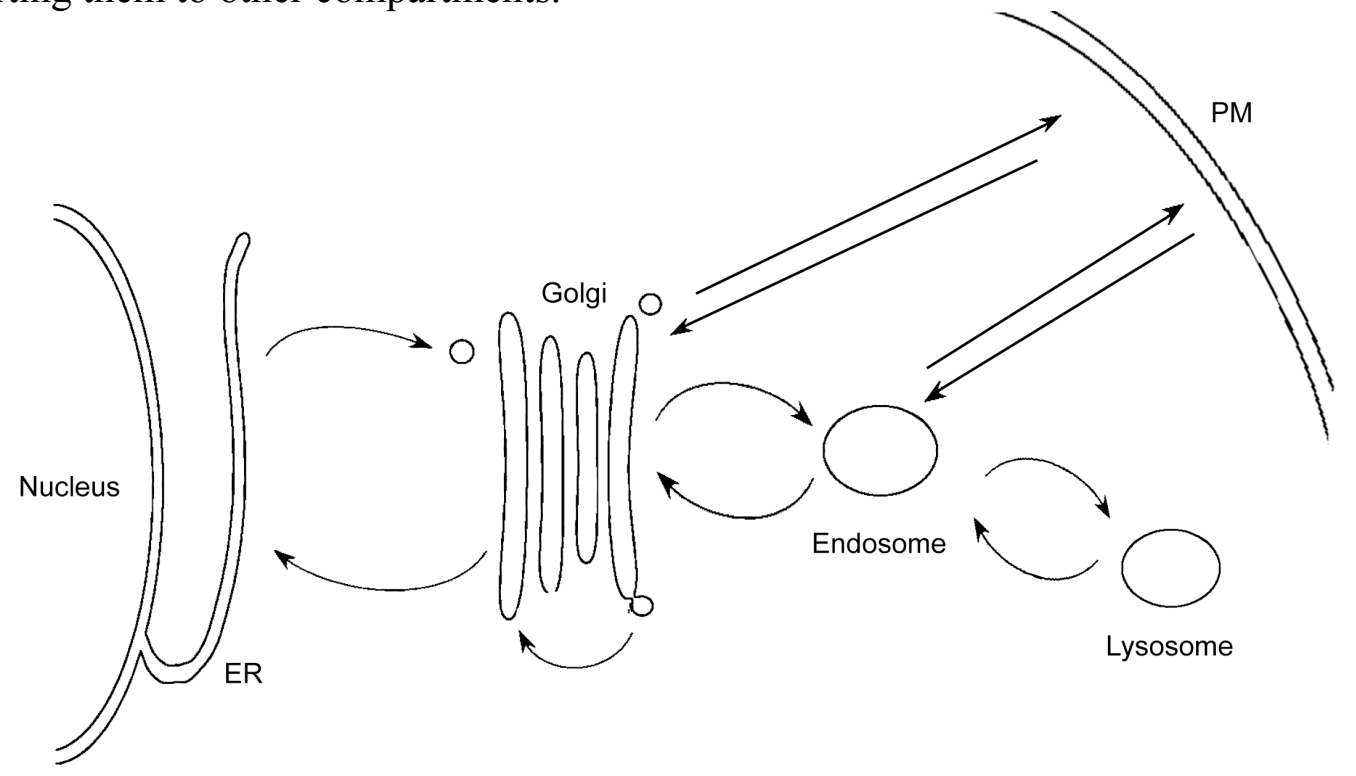

Figure II.1. The major sub-cellular compartments of eukaryotic cells. Eukaryotes have membrane bound compartments, which carry out specific functions.: ER, Golgi, Endosomes, Lysosomes. Proteins and membrane are continuously transported between these compartments and to the plasma membrane (PM). Source: Picture taken and adapted from Oka and Krieger, 2005. 
It is a polarized structure composed of multiple cisternae with the early cis-faced cisternae oriented towards the ER and the late trans-faced cisternae oriented away from the ER (Lowe, 2010; Glick and Nakano, 2009). Endosomes and lysosomes are cellular stations for recycling and degradation of biomolecules (Seaman, 2008; Alberts et al., 2008). Each compartment also maintains a highly specialized microenvironment to facilitate their functions. For example the ER has a highly oxidizing environment, which allows disulfide formation during protein folding and endosomes / lysosomes are acidic allowing for efficient function of degradative enzymes called hydrolases (Alberts et al., 2008).

The transport of proteins between these organelles is organized by membranebound vesicles. There are four essential steps in vesicle transport: vesicle budding from a donor compartment, vesicle motility, vesicle tethering and vesicle fusion to a target compartment (Fig II.2.). These processes demand the coordinated effort of a cohort of proteins: coat proteins, molecular tethering factors, monomeric guanosine nucleotide triphosphatases (GTPases) and soluble N-ethylmaleimide-sensitive factor attachment protein receptors (SNAREs) (Cai et al., 2007). Among these factors, monomeric GTPases have emerged as the major regulators due to their involvement in all steps of vesicular transport (Segev, 2011).

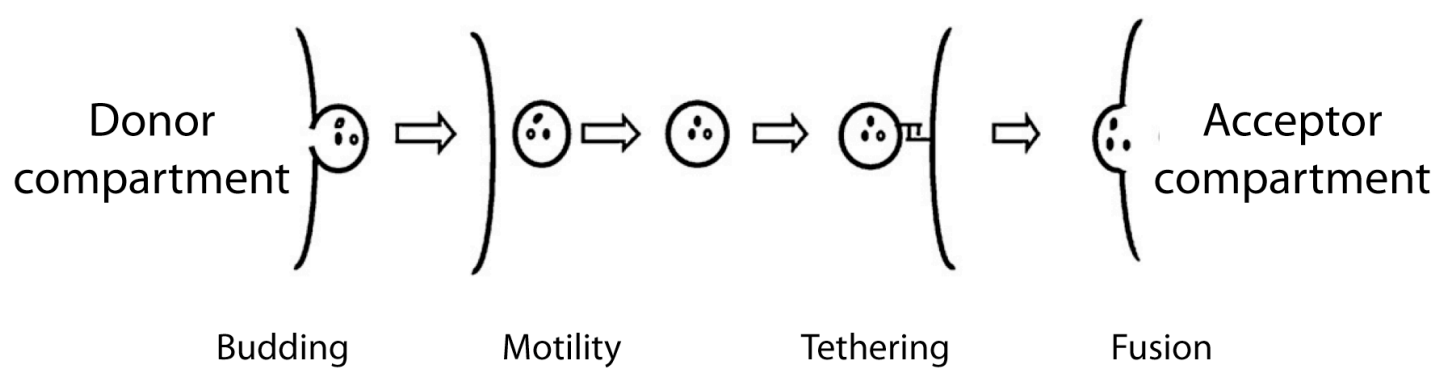

Figure II.2. Vesicular transport between organelles occurs in four distinct steps. Vesicles bud from the donor compartment through the recruitment of coat proteins (COPI, COPII and Clathrin). Cargo proteins are then loaded into newly formed vesicles and transported to the acceptor compartment via motor proteins. Upon arrival, vesicles are tethered to the acceptor compartment and subsequently undergo fusion. Monomeric GTPases of the Arf/Sar, Rho, Dynamin and Rab families control and regulate each of these steps. (Cai et al., 2007; Donaldson and Segev, 2009). Source: picture taken and adapted from Segev, 2011. 


\subsection{Monomeric GTPases}

Four major families of GTPases function to regulate intracellular transport: the Arf/Sar family, the Rho family, the Dynamin family and the Rab family (Donaldson and Segev, 2009; Itzen and Goody, 2011). The hallmark of these GTPases is that they act as molecular switches cycling between an active guanosine nucleotide triphosphate (GTP)-bound form and an inactive guanosine nucleotide diphosphate (GDP)-bound form (Fig. II.3). With the exception of dynamin, these GTPases require accessory enzymes named guanine nucleotide exchange factors (GEFs) to catalyze the exchange of GDP to GTP and GTPase activating proteins (GAPs) to catalyze the hydrolysis of GTP to GDP (Hinshaw, 2000; Itzen and Goody, 2011). In the active GTP-bound form, GTPases bind to a multitude of effector proteins (Takai et al., 2001). The complex array of GTPase-effector interactions allows them to participate at multiple steps in vesicular transport.

The Arf/Sar family of small GTPases $(\sim 20 \mathrm{kDa})$ is composed of 7 members: Arf1-Arf6 and Sar1. Members of this family are structurally distinguished by the presence of an N-terminal amphipathic helix. Normally buried, this helix is exposed upon binding to GTP allowing for localization to membranes (Gillingham and Munro, 2007). Most members of the Arf family are additionally myristoylated at the Nterminus (Wennerberg et al., 2005). These proteins are best characterized for their role in vesicle budding at the ER-Golgi interface. Arf1 localization to the Golgi leads to the recruitment of coat protein complex I (COPI) and facilitates membrane deformation during vesicle budding (Pucadyil and Schmid, 2009). In contrast to Arf1, Sar1 localizes to the ER and recruits components of the coat protein complex II (COPII) also facilitating membrane deformation during budding at ER exit sites. Furthermore, members of the Arf family have been proposed to act as timers promoting disassembly of COP coats post-vesicle budding (Barlowe et al., 1994; Gillingham and Munro, 2007).

The Rho family of small GTPases are composed of 3 members: RhoA, Rac and CDC42. Rho GTPases all share a conserved -CAAX motif at the C-terminus, which serves as a site for isoprenylation (Hakoshima, 2003). Once activated, this lipid modification allows Rho proteins to localize to membranes. Rho GTPases are involved in regulating many processes, but their best-documented role is in 
A

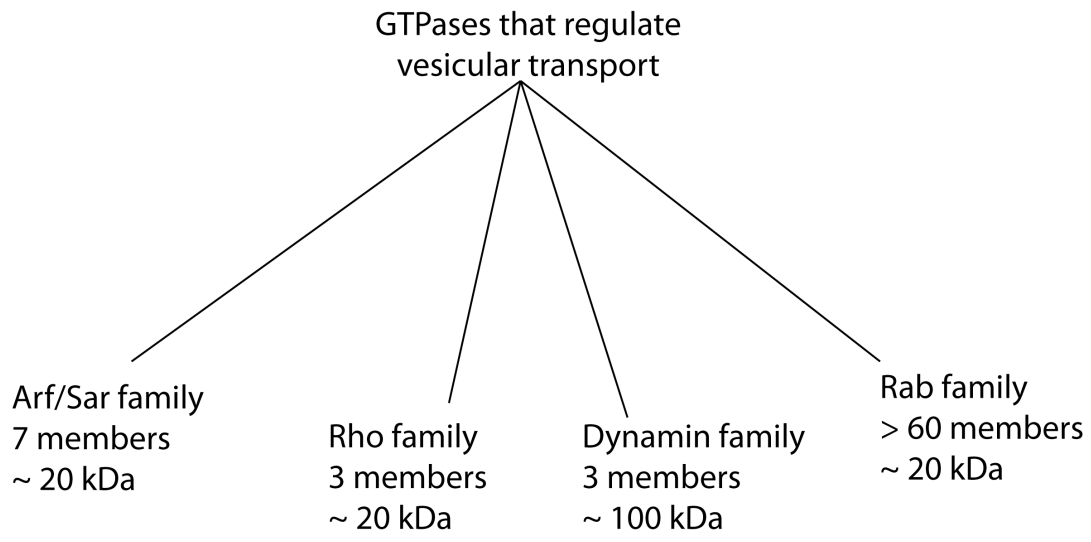

B

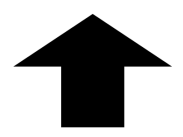

ON
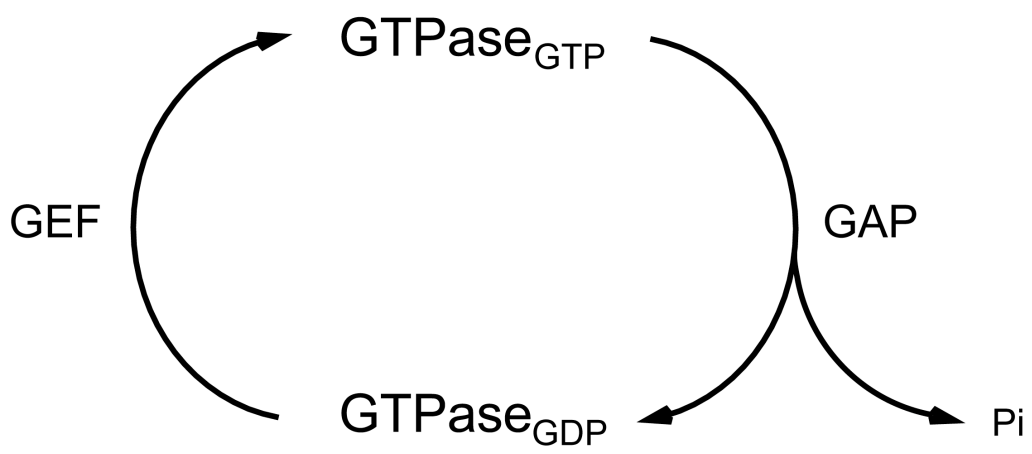

OFF

Figure II.3. Regulation of vesicular transport by GTPases. A. Four major GTPase families regulate vesicular transport: The Arf/Sar, Rho, Dynamin and Rab families. B. All GTPases cycle between an active GTP-bound form and an inactive GDP-bound form. Since most monomeric GTPases have slow intrinsic rates of GDP release and GTP hydrolysis they require the assistance of GEFs and GAPs to catalyze these reactions. (Goody and Hoffman-Goody, 2002). Pi: Inorganic phosphate

regulating actin dynamics within the cell. Rac GTPases stimulate the formation of plasma membrane extensions called lamellipodia, $\mathrm{Cdc} 42$ regulates the formation of finger-like protrusions called filipodia and Rho controls the formation of actin bundles such as stress fibers (Nobes and Hall, 1995). Constitutively active and inactive mutants of Rho GTPases also show defects in clathrin-mediated endocytosis of transferrin and epidermal growth factor (EGF) suggesting its role in vesicular transport (Lamaze et al., 1996). It has been proposed that this effect of Rho GTPases 
on endocytic trafficking is mediated via its modulation of the actin network. However, the precise mechanisms of such a function remains unknown (Symons and Rusk, 2003).

The dynamin family of GTPases is composed of 3 members: Dynamin 1-3. As a GTPase family, they are relatively large proteins $(\sim 100 \mathrm{kDa})$ that are composed of several domains: a GTP hydrolysis domain, a middle domain, a pleckstrin homology domain for membrane localization, a GTPase effector domain and a Cterminal proline-rich domain (Low and Löwe, 2010). Unlike the small GTPases, dynamins have high rates of intrinsic GTPase activity. This relieves them from the need for GAPs to regulate their function (Hinshaw, 2000). Once activated, they polymerize around the necks of newly budded vesicles and assist mainly in the pinching reaction that separates newly formed vesicles from the donor compartment (Hinshaw, 2000).

With over 60 members encoded in the human genome, the Rab family is by far the largest family of GTPases (Pereira-Leal and Seabra, 2001). Most Rabs have also been shown to localize to specific compartments creating the idea that they give identity to these compartments. Together with the fact that each Rab binds a vast number of effector proteins, this has raised much interest in unraveling their potential role as the master regulators of intracellular transport (Zerial and McBride, 2001).

\subsection{Principles of Rab GTPase structure}

\subsubsection{The Rab GTPase fold}

Being a sub-group of the Ras superfamily of GTPases, Rab proteins share the common GTPase fold consisting of six $\beta$-sheets ( $\beta 1-6)$ and five $\alpha$-helices $(\alpha 1-5)$.

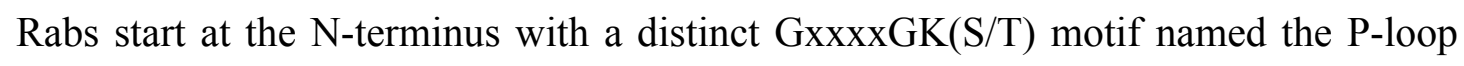
(loop between $\beta 1 \alpha 1$ ), which is important for binding phosphate and coordinating $\mathrm{Mg}^{2+}$. The GTPase fold additionally contains two switch regions (switch I and switch II), which stabilize the $\gamma$-phosphate of GTP (Hutagalung and Novick, 2011). In the GDP bound state, the switch regions are disordered, but upon GTP binding they undergo a conformation change to an ordered state (Fig II.4). This active conformation of the switch regions varies largely between Rabs and is thought to contribute towards functional specificity, along with the interswitch region and the 
A N-terminus:

Interswitch Switch 2

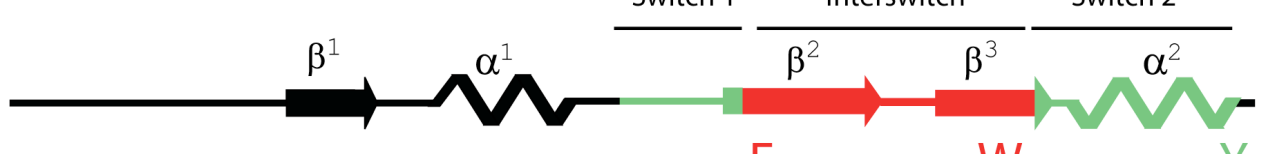

Rab5A:

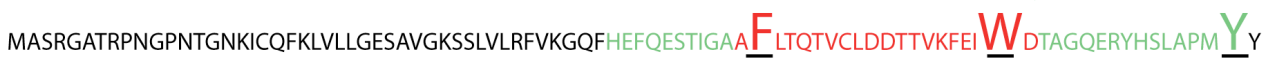
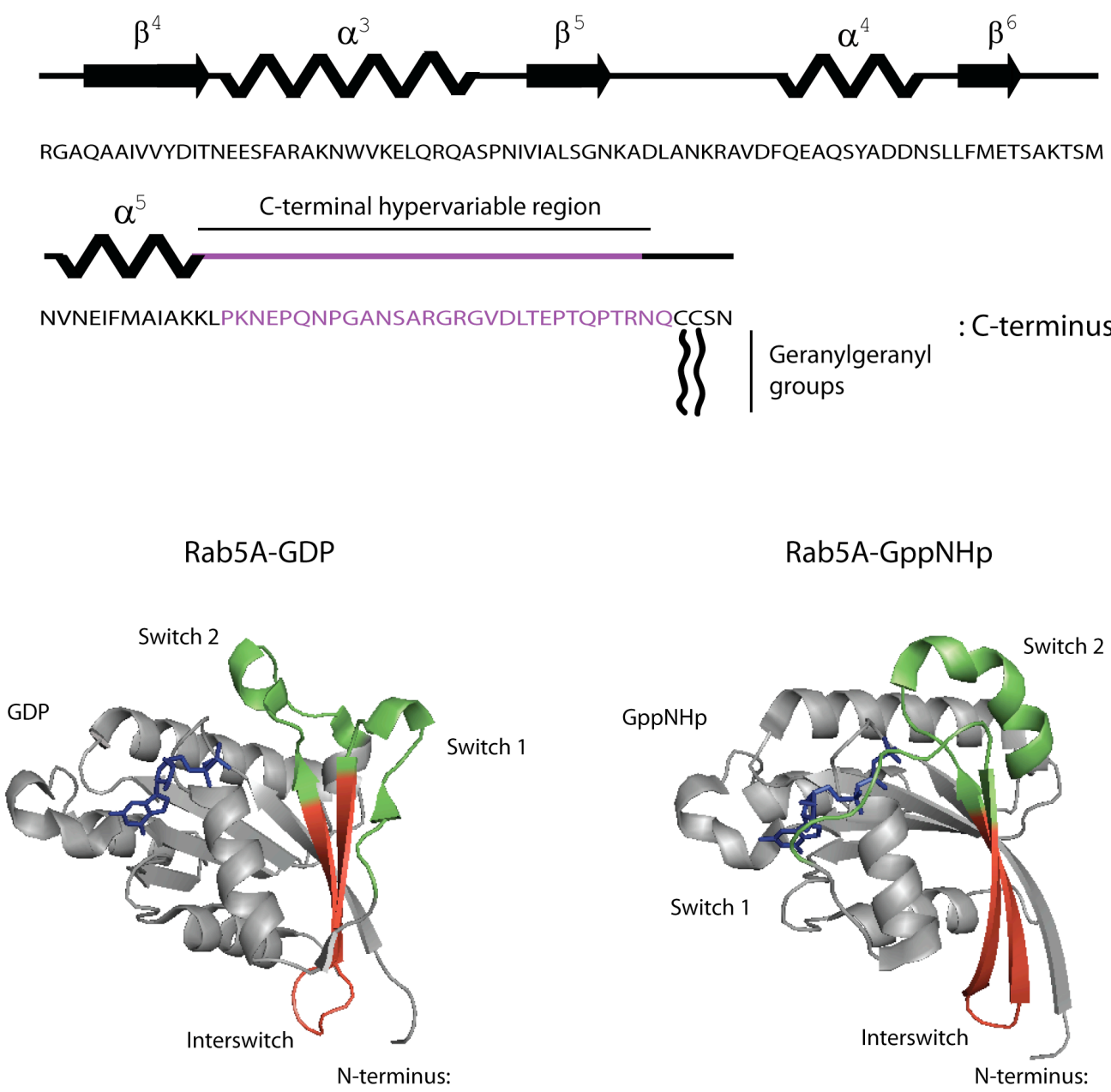

C

Figure II.4. Detailed structure of Rab GTPases.

A. Schematic representation of the secondary structure of the Rab GTPase: Rab5A. The switch (green) / interswitch (red) regions and $\alpha 3 \beta 5$ loop are important for Rab-Effector interactions. The hydrophobic triad (F57, W74 and Y89) are enlarged and underlined. The hypervariable region (purple) and the C-terminal cysteines are needed for localization (Hutagalung and Novick, 2011). B. Tertiary structure of the Rab5A bound to GDP and the non-hydrolyzable GTP analogue (GppNHp). Upon GTP binding, the switch I and switch II regions enter an ordered state (Lee et al., 2009). C. Scheme of a Rab protein attached to a membrane via its geranylgeranyl group. Source: Crystal structure of Rab5A-GDP and GppNHp (B) were taken and adapted from Zhu et al., 2004; Terzyan et al., 2004. Scheme of Rab bound to membrane (C) was taken and adapted from Gillingham and Munro., 2007. 
$\alpha 3 \beta 5$ loop (Lee et al., 2009). An individual Rab protein binds its cognate GAP, GEF and effector proteins via these distinct motifs (Itzen and Goody, 2011).

\subsubsection{Structural insights on Rab-GAP interactions}

RabGAPs stimulate the rate of GTP hydrolysis of Rabs. They are defined by a characteristic TBC (Tre-2/Cdc16/Bub2) domain. Over 40 members have been identified in the human genome (Fukuda, 2011). The crystal structure of the yeast GAP, Gyp1p, in complex with the mammalian GDP-Rab33-AlF 3 revealed that IxxDxxR and YxQ motifs of GAPs contribute to GTP hydrolysis through a dualfinger mechanism. Mutation of either the arginine or glutamine finger to alanine abolishes GAP activity (Pan et al., 2006). Unlike other small GTPases, the DxxGQ motif of switch II is not thought to participate directly in the catalysis by RabGAPs, but instead binds at another site on the GAP backbone (Fig II.5) (Pan et al., 2006). Although, the catalytic mechanism of GAP action has been elucidated, it is not fully clear how Rabs recognize their cognate GAPs.

A

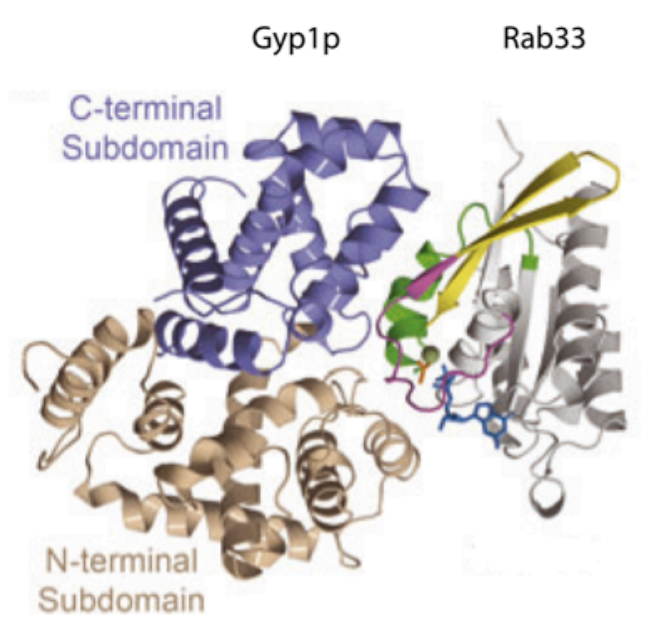

B

Active site of Gyp1p-GDP-Rab33-AIF 3

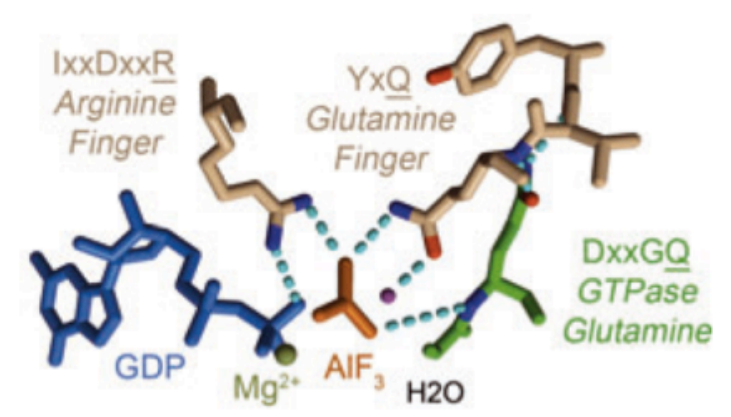

Figure II.5. Structure of a Rab-GAP complex in its transition state. A. Overview of the GyplpGDP-Rab33 complex in the presence of the transition state mimetic $\mathrm{AlF}_{3}$. B. Selected view of the active site of the complex displaying the catalytic IxxDxxR and YxQ motifs of Gyplp. The arginine and the glutamine residues make contact with $\mathrm{AlF}_{3}$, whereas the glutamine of the DxxGQ motif from Rab33 does not. Source: Picture is taken from Pan et al., 2006.

\subsubsection{Structural insights on Rab-GEF interactions}

RabGEFs catalyze the exchange of GDP to GTP by increasing the rate of GDP dissociation of Rabs. In addition, it has also been shown that they contribute to Rab 
localization by locally activating and concentrating them (Schoebel et al., 2009). To date, approximately 25 RabGEFs have been identified to be encoded by the human genome (Barr and Lambright, 2010). However, in contrast to RabGAPs, there is no major consensus domain for RabGEFs making it difficult to identify them. They exist in many shapes and sizes: Vps9-domain containing GEFs such as Rabex5, the GEF for Rab5, operate by binding to the switch/interswitch regions, opening switch I and inserting an aspartate finger to destabilize the $\mathrm{Mg}^{2+} /$ phosphate binding sites (Itzen and Goody, 2011). Coiled coil domain containing GEFs such as Sec2, found in yeast, act on the Rab GTPase Sec4, through a similar mechanism of binding to the switch/interswitch region and distorting switch I (Barr and Lambright, 2010). Recently a third group of DENN (differentially expressed normal versus neoplastic) domain containing GEFs have been discovered, however their mechanism of action remains unclear. It is likely that they also function via switch I displacement (Barr and Lambright, 2010; Sato et al., 2008; Yoshimura et al., 2010).

\subsubsection{Structural insights on Rab-Effector interactions}

Rab proteins bind a plethora of structurally unrelated effector proteins to carry out downstream functions. Much of the structural data available on Rab-Effector interactions suggest that the interaction interface occurs between one or two alphahelices / coiled coils of an effector and the switch/interswitch/ $\alpha 3 \beta 5$ loop of the Rab as seen with Rab3A-Rabphilin-3A, Rab4-Rabenosyn-5, Rab5A-Rabaptin-5C, Rab6GCC185, Rab7-RILP and Rab11-FIP2 (Ostermeier and Brunger, 1999; Eathiraj et al., 2005; Zhu et al., 2004; Burguete et al., 2008; Jagoe et al., 2006; Wu et al., 2005). Some of these structures are depicted in Figure II.6. Interestingly, all Rab-Effector interactions demand the requirement of a conserved hydrophobic triad of residues: a phenylalanine at the beginning of the interswitch region, a tryptophan in the second half of the interswitch region and a tyrosine at the end of switch II (shown for Rab5A in Fig.II.4). Among these residues, the phenylalanine helps coordinate $\mathrm{Mg}^{2+}$, while the tryptophan and tyrosine interact directly with the effector protein (Itzen and Goody, 2011).

Currently only a fraction of Rab-effector complexes have been crystallized. From genetic and biochemical studies, it is clear that rabs also bind to cargo molecules, kinases, phosphatases, motor proteins and large multi-subunit tethering 
A

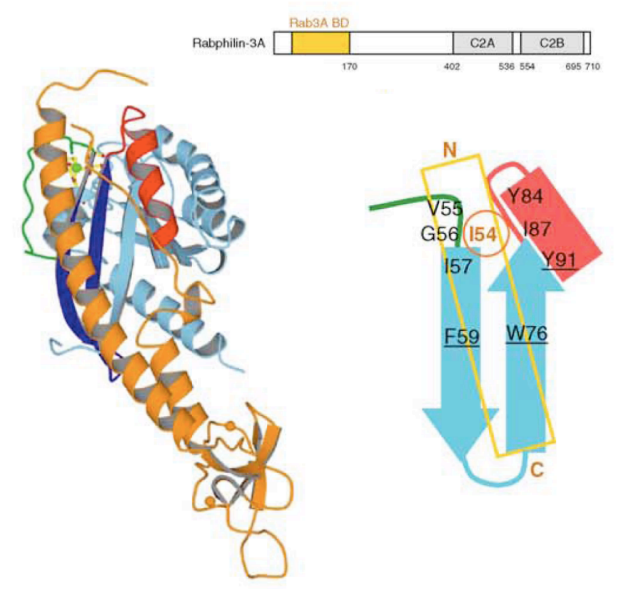

Rab3A-Rabphilin-3A

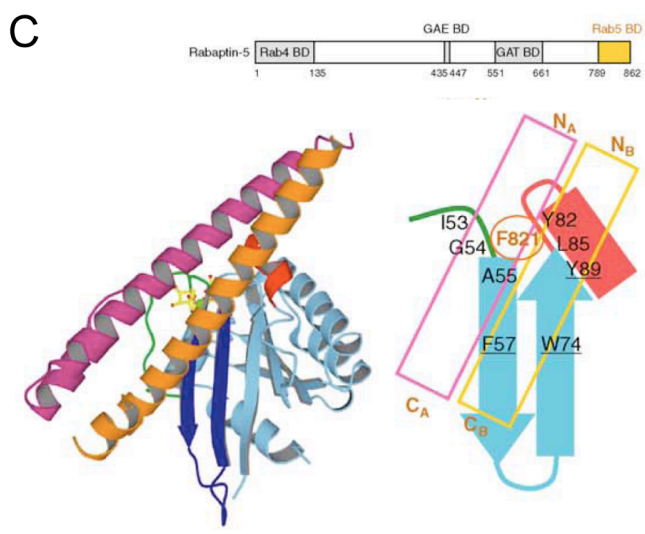

Rab5A-Rabaptin-5C
B

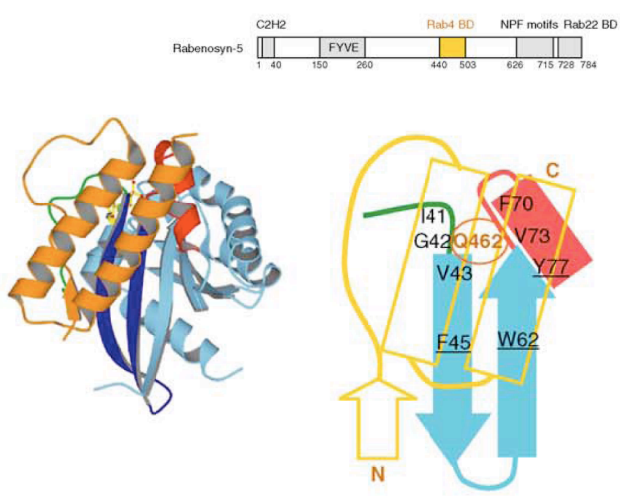

Rab4-Rabenosyn-5
Figure II.6. Variations in Rab-Effector complexes. Crystal structures of Rab3ARabphilin-3A, Rab4-Rabenosyn-5 and Rab5ARabenosyn-5C are depicted along with magnification of the interaction interface between effector (orange/pink) and switch/interswitch (blue) regions. The effector residue for Rab binding is circled. The hydrophobic triad residues of the Rab are underlined. A. Rabphilin-3A interacts with Rab3 through a single alpha helix motif. B. Rabenosyn-5 interacts with Rab4 through a helix-turn-helix motif and C. Rabaptin$5 \mathrm{C}$ interacts with Rab5A through a homodimeric coiled coil with the stoichiometric ratio of 2 coils : 2 Rab5 molecules (only one Rab5A molecule shown). Source: Pictures taken and adapted from Kawasaki et al., 2005.

complexes (Grosshans and Novick, 2006; Guo et al., 1999; Siniossoglou and Pelham, 2002). However, the nature of these interactions remains to be understood at atomic resolution.

\subsubsection{Structural insights on Rab localization}

Downstream of the switch regions necessary for GAP, GEF and effector interactions, is a C-terminal hypervariable region (Fig II.4). The hypervariable region of a Rab is the most divergent part of its structure and has hence been proposed to act as a targeting signal (Chavrier et al., 1991). Recent evidence has suggested that the localization of Rabs is more complex and dependent on other elements of its structure as well as on its interaction with effectors (Ali et al., 2004; Schoebel et al, 2009). Lastly, the C-terminus of rab proteins contains a characteristic cysteine motif (CC, CXC, CCX, CCXX or CCXXX), a target for geranylgeranylation. De novo 


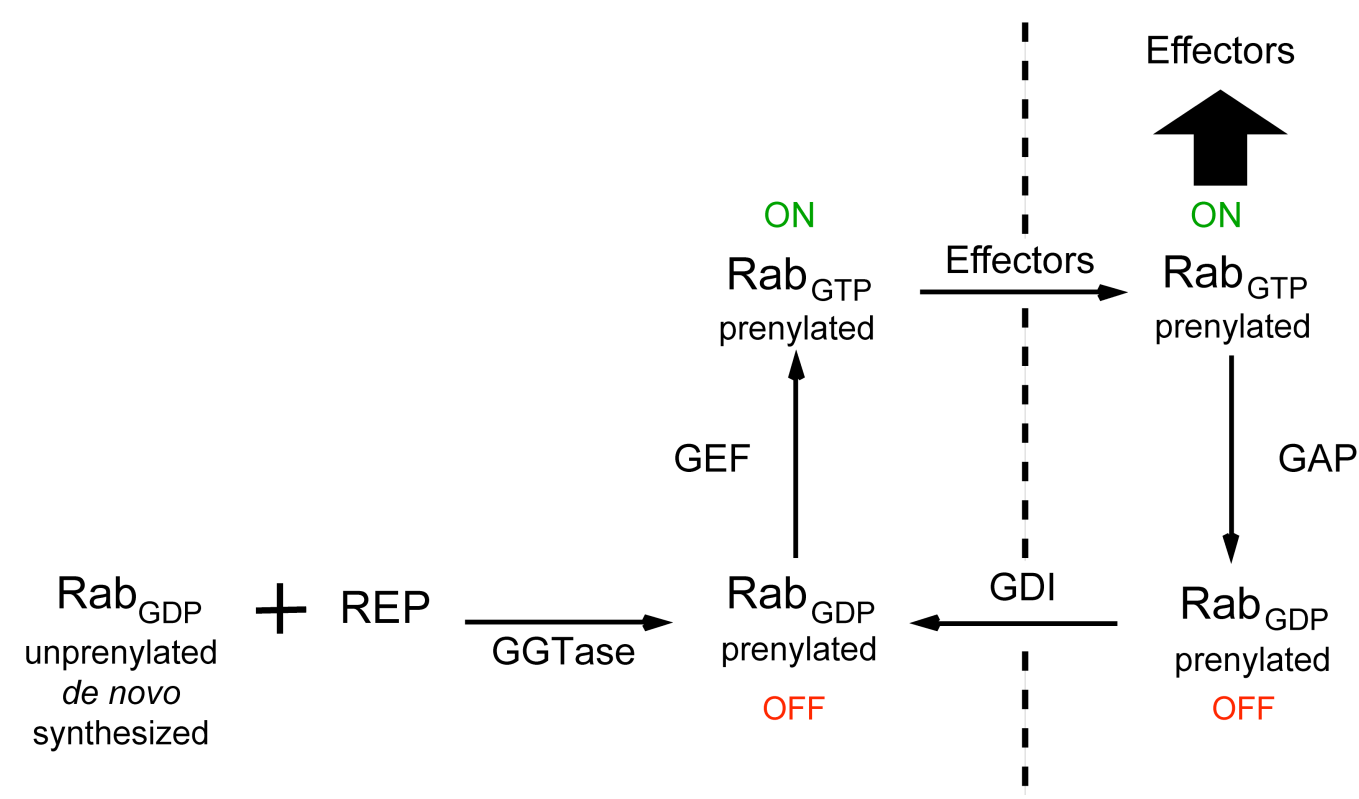

Figure II.7. Role of REP, RabGGTase and RabGDI on Rab localization. REP, RabGGTase and RabGDI are additional factors, which contribute to the localization and function of Rabs. Newly synthesized Rabs are recognized by REP and delivered to the RabGGTase for prenylation. After prenylation, they are inserted into the donor compartments. A simplistic model suggests that upon activation by RabGEFs and interaction with effectors they mediate vesicular transport to an acceptor compartment. At the acceptor compartment Rabs are inactivated by RabGAPs. Upon inactivation, RabGDIs scoops GDP bound Rabs out of the acceptor compartment and deliver them back to the donor compartment (Hutagalung and Novick, 2011).

synthesized Rabs are recognized by Rab Escort Protein (REP) and taken to the rab geranylgeranyl transferase (RabGGTase) where these cysteine residues are lipid modified. After modification REP escorts the prenylated Rab to its appropriate compartment (Wennerberg et al., 2005). Once inactivated, a molecule structurally similar to REP named Rab GDP dissociation inhibitor (GDI) is necessary to recycle GDP bound Rabs to their original compartments (Fig. II.7) (Hutagalung and Novick., 2006).

\subsection{Principles of Rab GTPase function}

\subsubsection{Rabs define sub-cellular compartments}

One of the distinguishing features of Rab GTPases is that they provide a blueprint of all major sub-cellular compartments. The following are several examples: Rab1 localizes to ER-Golgi intermediate compartments (ERGIC), Rab2/Rab6/Rab33 


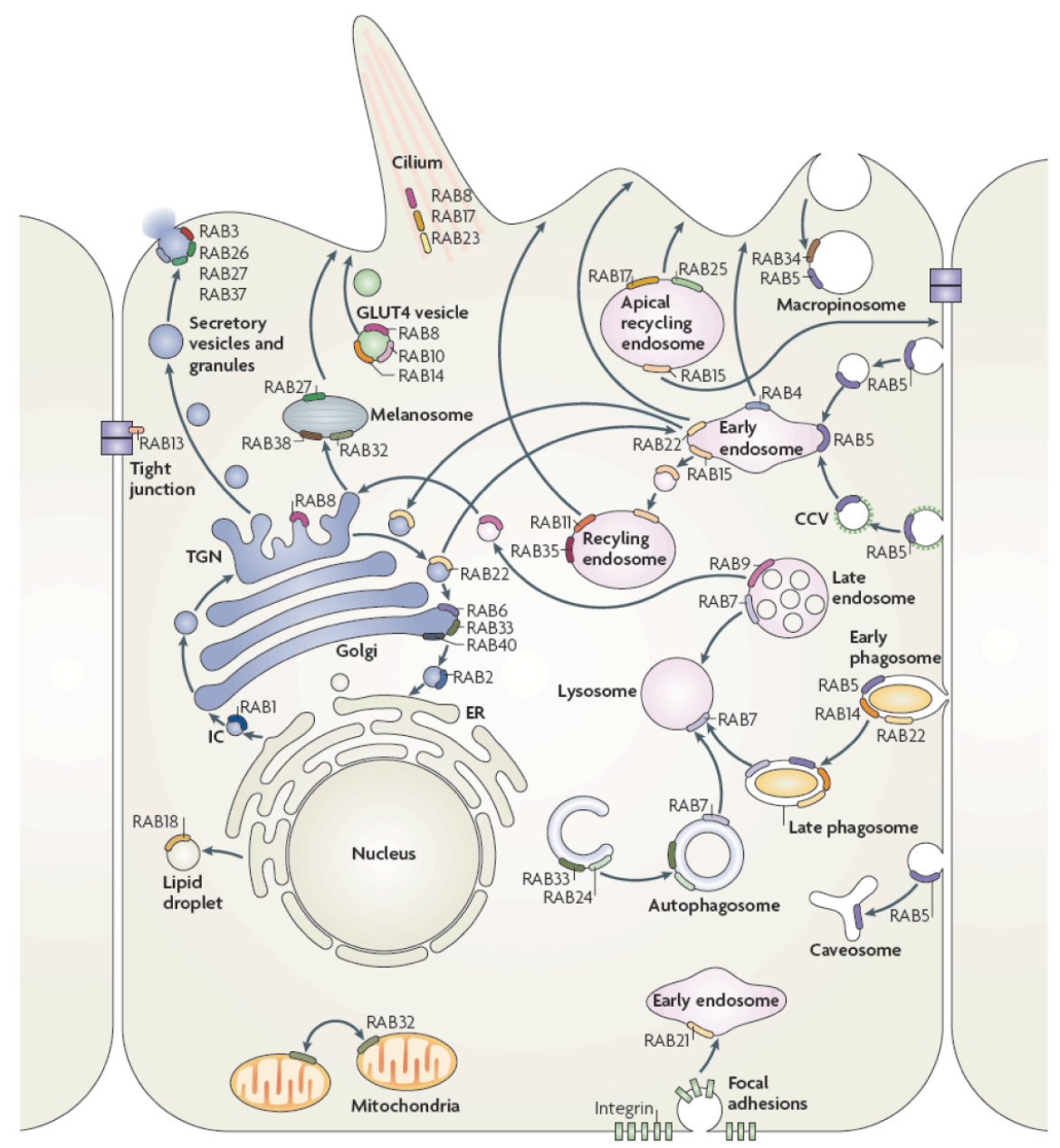

Figure II.8. Rabs localize to a variety of compartments. There are over 60 rabs encoded in the human genome. Most of these Rabs localize to specific compartments and regulate the transport of membrane and proteins from a donor compartment to an acceptor compartment. Often a single Rab has multiple localizations depending on the tissue that it is expressed. Source: Picture taken from Stenmark, 2009.

to the Golgi, Rab9 to the TGN (trans-Golgi network), Rab3/Rab27 to synaptic vesicles, Rab8 to the basal bodies, Rab10, Rab11 and Rab35 to recycling endosomes, Rab5 to early endosomes, Rab7 to late endosomes, and Rab18 to lipid droplets etc. (Figure II.8) (Babbey et al., 2006; Fischer von Mollard et al., 1990; Kouranti et al., 2006; Meresse et al., 1995; Nachury et al., 2007; Ozeki et al., 2005; Stenmark et al., 1994; Ullrich et al., 1994; Stenmark, 2009). Furthermore, the localizations of Rabs can differ between cell-types adding an additional level of complexity. For example, Rab10 localizes to Golgi/ endosomes in Madin-Darby Canine Kidney (MDCK) cells, to cilia in renal epithelial cells, to glucose transporter 4 storage vesicles (GSVs) in adipocytes and to synaptic vesicles (SVs) in hippocampal neurons (Schuck et al., 
2007; Babbey et al., 2006; Babbey et al., 2010; Larance et al., 2005; Sano et al., 2008; Pavlos et al., 2010). These diverse and intricate patterns of sub-cellular localizations allow Rabs to regulate all steps of intracellular trafficking ranging from budding to fusion.

\subsubsection{Role of Rab GTPases in vesicle budding and uncoating}

As described previously, vesicle budding is primarily controlled by the Arf GTPase family. However, there are certain cases where Rabs take on specific roles. Lysosomal hydrolases are synthesized in the ER and transported via the secretory pathway to lysosomes. In order for these enzymes to reach the destination, they must undergo an N-linked mannose-6-phosphate (M6P) attachment at the Golgi. Once modified, the hydrolases are recognized by M6P receptors, which transport the hydrolases to endo-lysosomes, release them and recycle back (Alberts et al., 2008). The recycling of M6P receptors back to the Golgi was found to be mediated by a cytosolic protein named tail-interacting protein of $47 \mathrm{kDa}$ (TIP47), which binds the receptors on their cytosolic domain (Diaz and Pfeffer, 1998). Interestingly, Rab9 was found to bind TIP47 leading to a model where Rab9 acts as an initiator of vesicle formation for M6P receptor recycling (Carroll et al., 2001).

Another example of Rabs participating in vesicle formation is seen through the interaction of Rab7 with the retromer complex. The retromer is involved in the transport of cargo from endosomes to the Golgi. It consists of sorting nexins and the Vps26-Vps29-Vps35 trimer, which binds directly to cargo (Seaman et al., 1998). The trimer is an effector of Rab7. Depletion of Rab7 leads to the disassembly of retromer and defects in endosome to Golgi trafficking (Rojas et al., 2008).

Newly budded vesicles must shed their coats (COP I, COPII or clathrin) upon budding to allow for competent fusion to an acceptor compartment (Hutagalung and Novick, 2011). Recently, a new role of Rab5 in clathrin uncoating was established. During clathrin coat formation, the clathrin adaptor protein, AP2, is recruited to membranes containing phosphatidylinositol-4,5-bisphosphate $\left(\mathrm{PIP}_{2}\right)$ (Owen, et al., 2004). Upon recruitment, the $\mu 2$ subunit of AP2 is phosphorylated by a $\mu 2$ kinase (AAK1). Kinase activity is required for cargo recruitment (Jackson et al., 2003). After clathrin coat formation, a Rab5 GEF named hRme-6 displaces AAK1 leading to 
decreased levels of phospho- $\mu 2$. This is likely to occur through the activation of Rab5 and recruitment of an effector with phosphatase activity (Semerdjieva et al., 2008).

\subsubsection{Role of Rab GTPases in vesicle motility}

Vesicles are transported from the donor compartment to the acceptor compartment through the cellular cytoskeleton. In yeast, the Rab, Ypt31/Ypt32, localizes to the Golgi and to Golgi derived secretory vesicles. It has been shown that Ypt31/Ypt32 interacts directly with the type V myosin motor, Myo2. Since Myo2 is an actin-based motor, this interaction allows Golgi derived vesicles to move, via the actin cytoskeleton, towards the plasma membrane tip of budding yeast cells (Lipatova et al., 2008). Although, initially the transport of these vesicles was thought to be mediated by the Rab, Sec4, a direct interaction between Sec4 and Myo2 remains to be elucidated (Wagner et al., 2002). Mammalian Rabs have also been shown to regulate vesicle motility, albeit through indirect interactions with motors. Rab11 binds to the C-terminus of an effector called FIP2 (Rab11 family interacting protein-2). FIP2 consequently binds myosin $\mathrm{Vb}$ at a site closer to its $\mathrm{N}$-terminus. This ternary complex is suggested to be important for vesicular transport from recycling endosomes to the plasma membrane (Hales et al., 2002). Similarly, Rab27 also interacts with myosin

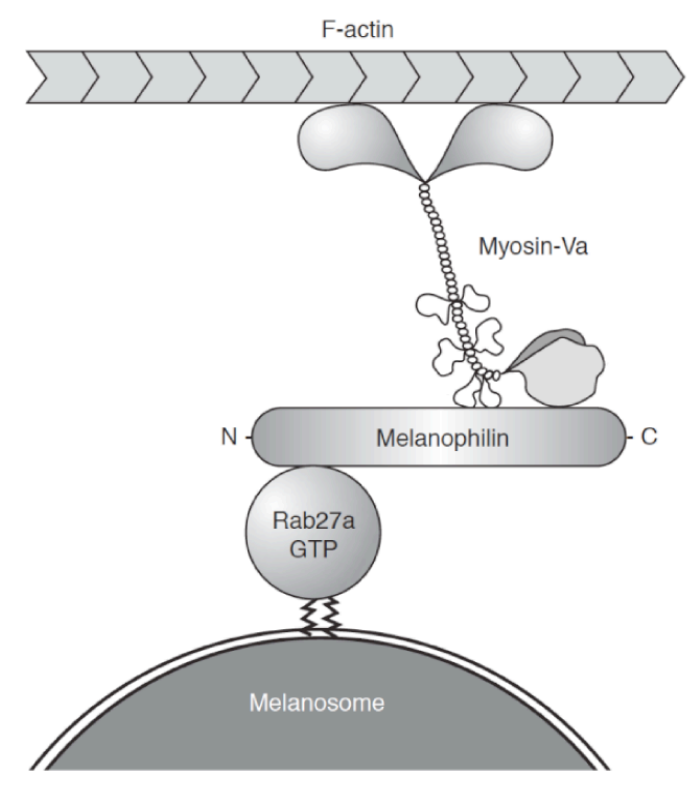

Figure II.9. Rabs interact with motor proteins. In melanocytes, Rab27 localizes to lysosome related organelles named melanosomes. Melanosomes contain the pigment melanin, which protects skin cells against UV radiation. Melanosomes are trafficked to the cell periphery via an indirect interaction of Rab27a with the myosin Va motor protein. This interaction is mediated through the synaptotagminlike protein, melanophilin (Fukuda et al., 2002). Source: picture taken and modified from Wu et al., 2002. 
$\mathrm{Va}$, through an adaptor protein named melanophilin to regulate the transport of melanosomes to the cell periphery (Fig II.9) (Wu et al., 2002). Mutations in Rab27, myosin $\mathrm{Va}$ and melophilin are associated with Griscelli syndrome a pigmentary disorder of the skin resulting from perturbed melanin trafficking (Menasche et al,. 2000; Van Gele et al., 2009).

Rab mediated motility is not restricted to the actin cytoskeleton. Several Rabs have also been shown to interact with the microtubule motor proteins: kinesin and dynein / dynactin complex. Rab6 binds to the kinesin-like motor Rabkinesin-6 to regulate intra-Golgi transport (Echard et al., 1998). Rab7 interacts indirectly to dynein / dynactin via forming a complex with Rab-interacting lysosomal protein (RILP) and oxysterol-binding protein-related protein 1L (ORP1L) complex (Johansson et al., 2007). Interestingly, certain pathogens such as Salmonella enterica prevent RILP recruitment by Rab7 to modulate endo-lysosomal trafficking while entering host cells (Harrison et al., 2004).

\subsubsection{Role of Rab GTPases in vesicle tethering}

Specificity and directionality in vesicular transport is mediated by highly regulated tethering of vesicles to the acceptor compartment. Among all roles of Rab GTPases in vesicular transport, its role in vesicle tethering is by far the most pronounced. Rabs bind to two major classes of tethering factors: long coiled-coil proteins and large multisubunit protein complexes.

Golgins are the most prominent member of the long coiled-coil class of proteins. Golgins localize to distinct Golgi cisternae and are thought to form a proteinacous meshwork surrounding it ( $\mathrm{Yu}$ and Hughson, 2010). They extend long homodimeric-coiled coils away from the Golgi membrane, which serve as sites for Rab GTPase binding. Additionally they typically have C-terminal GRIP/GRAB domains, which are recognized by Arf and Arl (Arf-like) GTPases (Ramirez and Lowe, 2009). A model for the interaction of the TGN-localized Golgin, GCC185 with both GTPases was recently published (Fig. II.10) (Burguete et al., 2008). The precise sequence of events, which takes place, still remains elusive. The most recent hypothesis of Golgin function, suggests that they are targeted to the Golgi through the interaction of their GRIP domains with Arfs and that they use their coiled coil domains as tentacles to tether incoming vesicles by contacting Rab GTPases (Sinka et al., 2008). 
A
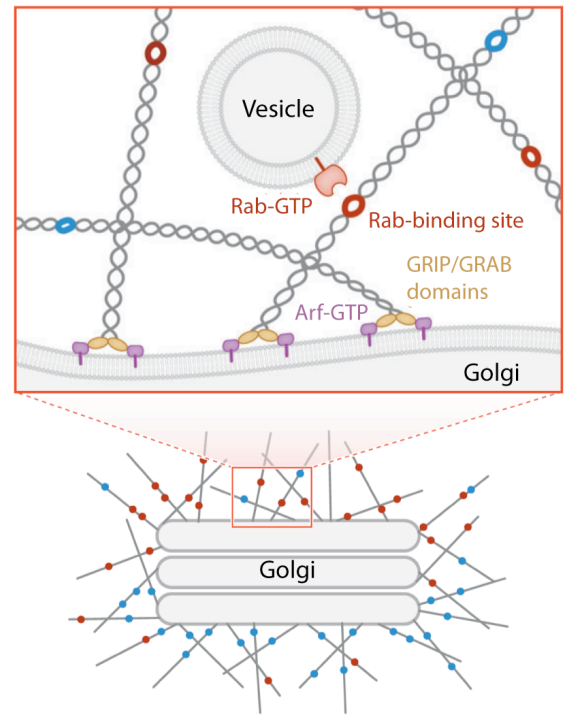

B

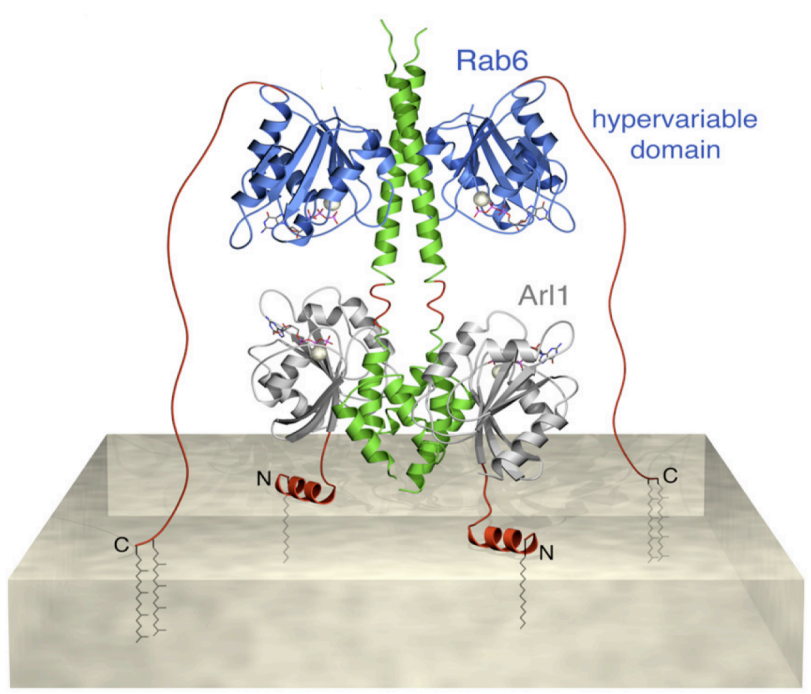

Figure II.10. Model for tethering by Golgins. A. Since Golgins form a meshwork around the Golgi and because they harbor multiple binding sites for Rabs, it is currently believed that they behave like tentacles attracting incoming vesicles from donor compartments. Golgins contain N-terminal GRIP domains, which bind Arfs and C-terminal coiled coils, which bind Rabs. (Yu and Hughson, 2010) B. A model of the entire Rab6-GCC185-Arl1 complex based on the crystal structures of Rab6-GCC185 and Arl1-GCC185. Source: Pictures taken from Yu and Hughson, 2010; Burguete et al., 2008.

There are seven conserved multisubunit tethering complexes identified: COG (conserved oligomeric complex), CORVET, Ds11, exocyst, GARP (Golgi associated retrograde protein complex), HOPS (homotypic vacuole protein sorting) and TRAPP (Transport protein particle complex) (Cai et al., 2007). Among these complexes, several have been shown to interact with Rabs. One of the first large multisubunit complex - Rab interaction was identified in yeast between Sec4 and the Sec15 subunit of the exocyst. This interaction is important for vesicle tethering and polarized exocytosis at the bud tip (Guo et al., 1999). Furthermore, in yeast, a subunit of COG interacts with the GTPase Yptlp as well as the COPI coat to regulate recycling of Golgi resident enzymes to their rightful cisternae (Suvorova et al., 2002; Hutagalung and Novick, 2011). The Vps52 subunit of the GARP also interacts with Rab6 to regulate retrograde transport from the endosomes to Golgi (Liewen et al., 2005; Siniossoglou and Pelham, 2001). Unlike other complexes, the TRAPP and HOPS complexes act as GEFs for Rab1 and Ypt7p (yeast orthologue of Rab7) respectively (Yamasaki et al., 2009; Wurmser et al., 2000). However, it must be mentioned that the claim of HOPS mediated GEF activity on Ypt7p is a subject of recent controversy (Nordmann et al., 2010). It has also been suggested that CORVET may act as a GEF on Vps21 (yeast orthologue of Rab5) (Peplowska et al., 2007). 


\subsubsection{Role of Rab GTPases in vesicle fusion}

SNAREs were believed to form the minimal machinery necessary for vesicle fusion. In vitro, the neuronal SNAREs; Synaptobrevin-2, SNAP-25 and syntaxin-1, were shown to autonomously fuse membranes. However, these reactions displayed slow kinetics (half-life of 10 minutes) and required relatively high concentrations of SNAREs (mg range) (Weber et al., 1998). Since, in vivo, these reactions occur in the sub-millisecond time scale and given that as few as 3 SNARE complexes are needed for fast vesicle fusion, it is conceivable that additional factors are necessary for efficient neuronal membrane fusion (Katz et al. 1969; Mohrmann et al., 2010). Interestingly, in line with this argument, two recent studies have already provided evidence for the integral role of Rabs in endo-lysosomal homotypic fusion. Rab5 and its effectors, Rabenosyn-5 and Rabaptin-5, have been shown to be essential for synthetic endosomes fusion bearing endosomal SNAREs (Ohya et al., 2009). The yeast orthologue of Rab7, Ypt7p, and its GEF, the HOPS complex are necessary to drive fusion of proteoliposomes bearing yeast vacuolar SNAREs (Stroupe et al., 2009). It is important to note that even though Rabs are essential for fusion, it is likely through their interactions with tethering factors that they increase the efficiency and specificity of SNARE driven vesicle fusion.

\subsection{Integration of Rab function}

Although many Rabs have been shown to localize to distinct compartments for their functions, it is clear that the number of Rabs far exceed the number of cellular compartments. This leads to multiple Rabs localizing to the same compartment. This phenomenon has brought forth the concept of the fine localization of Rabs to microdomains and the maturation of these domains via Rab cascades (for a recent review see Stenmark, 2009).

\subsubsection{Rabs form microdomains}

Pioneering studies from Zerial and colleagues, showed, for the first time, that Rab4, Rab5 and Rab11 localize to distinct domains on endosomes (Sönnichson et al., 2000). Early endosomes predominantly consist of Rab5 and Rab4 domains, with few Rab11 domains, whereas recycling endosomes contain mostly Rab4 and Rab11 


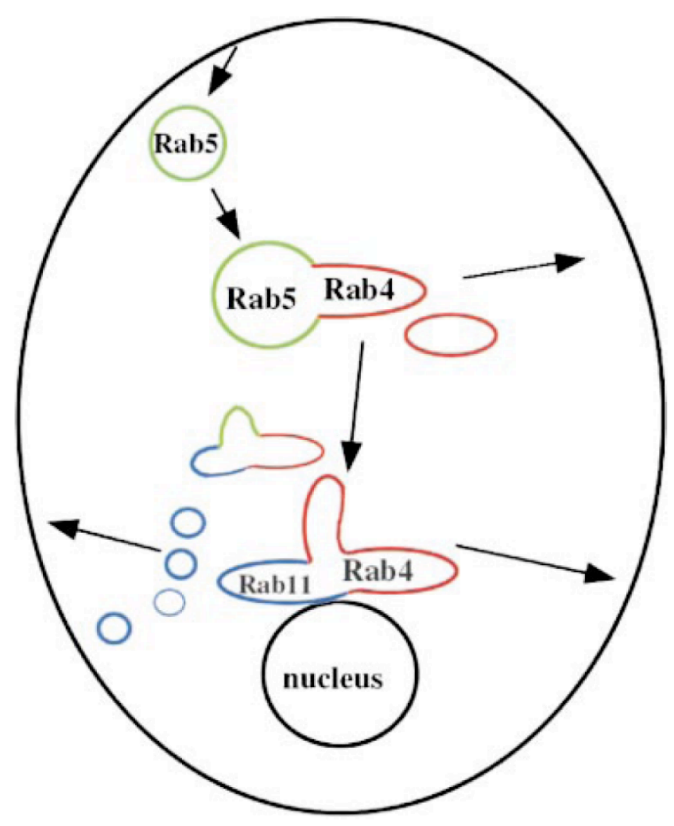

Figure II.11. Rabs localize to microdomains. It has been shown that Rab proteins, which localize to the same compartment, often exist in microdomains. This is seen with Rab4 and Rab5, which localize to microdomains on early endosomes. It is also evident with Rab11 and Rab4 on recycling endosomes. Source: Picture taken from Sönnichson et al., 2000.

domains (Fig. II.11) (Sönnichson et al., 2000). Through results from transferrin recycling assays, it was shown that newly endocytosed transferrin accumulates in Rab5-Rab4 positive compartments and then upon saturation enters the Rab11-Rab4 positive compartments (Sönnichson et al., 2000).

In an independent study, it was also revealed that Rab9 and Rab7 localize to distinct microdomains on late endosomes. It is believed that Rab9 positive microdomains function in M6P receptor recycling to the Golgi, whereas the Rab7 microdomains continue on to undergo homotypic fusion (Barbero et al., 2002). Although it must be mentioned that a recent study established a role for Rab7 also in M6P receptor recycling to the Golgi via an interaction with retromer (Rojas et al., 2008). However, it is believed that there are two pools of Rab7, one pool of Rab7 localized at the early endosomes in the proximity of Rab5 and another pool of Rab7 localized at the late endosomes in the proximity of Rab9, which would explain these conflicting data. 


\subsubsection{Rab conversion cascades}

The existence of microdomains allows for the spatial segregation of Rabs on a single compartment. This, in turn, permits efficient sorting of cargos. Recently, it has come to light that an individual Rab-microdomain can temporally mature into another Rab-microdomain. This gives rise to the idea of compartmental maturation by Rab cascades. Rab conversion is a type of cascade where an individual Rab recruits the GEF of a secondary Rab thereby activating it and initiating the next trafficking step (Figure II.12) (Stenmark, 2009). Such cascades are thought to provide directionality in trafficking.

The first Rab conversion was described, in yeast, between the GTPases; Ypt31/32 and Sec4. Ypt32/31 localizes to the late Golgi. However, as secretory vesicles form at the TGN, Ypt32/31 recruits Sec2. Sec2 is a GEF, which subsequently recruits and activates its substrate Sec4. Sec4 labeled secretory vesicles then move towards their acceptor membrane (Ortiz et al., 2002). A second Rab conversion between Rab5 and Rab7 has also been elucidated. Early Rab5 positive endosomes recruit the HOPS complex through an interaction between Rab5 and Vps41 (a subunit of HOPS). Vps39, another subunit of HOPS harboring GEF activity, is thought to recruit and activate $\mathrm{Rab} 7$ allowing for maturation of early endosomes into late endosomes (Rink et al., 2005). Recently a new complex named, Mon1-Ccz1 has been implicated in this conversion. Interestingly Mon1-Ccz1 binds and displaces Rabx5 (GEF for Rab5) and in parallel binds to the Vps39 subunit of HOPS, perhaps contributing to the fidelity of this conversion (Poteryaev et al., 2010; Nordmann et al., 2010).

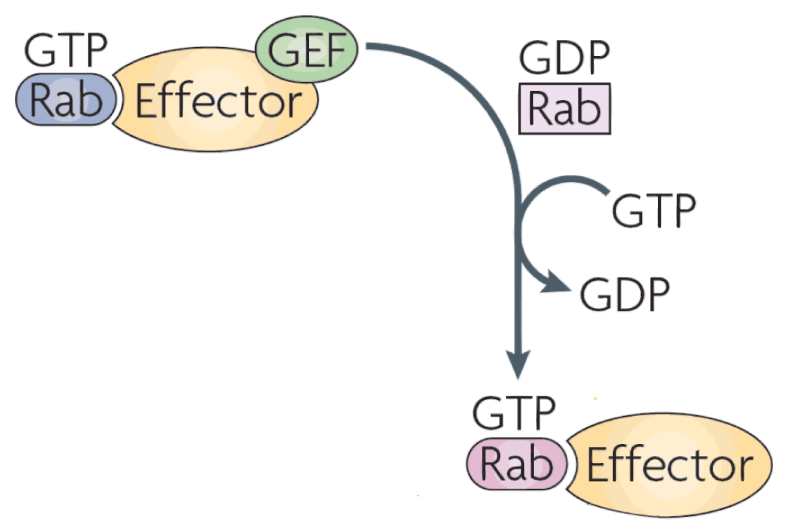

Figure II.12. Model of a Rab conversion cascade. Rabs label compartments and as one compartment matures into another compartment, a specific label is exchanged for another through Rab cascades. Rab conversion occurs when an upstream Rab recruits the GEF of a downstream Rab. Source: Picture taken and adapted from Stenmark, 2009. 


\subsubsection{Rab exclusion cascades}

During the process of compartmental maturation, not only is it important to recruit a new Rab, but also to displace the previous upstream Rab. This is achieved by a process whereby the downstream Rab recruits a GAP to inactivate the upstream Rab. An example of such a cascade is seen also in yeast between Ypt1 and Ypt31. Early Golgi compartments labeled with Ypt1 eventually mature into Ypt31 positive compartments. To accomplish this, Ypt31 recruits Gyp1, the GAP for Ypt1p, to exclude Ypt1 from Ypt31 positive membranes (Rivera-Molina and Novick, 2009). Figure II.13 illustrates how such a Rab exclusion cascade would look like.

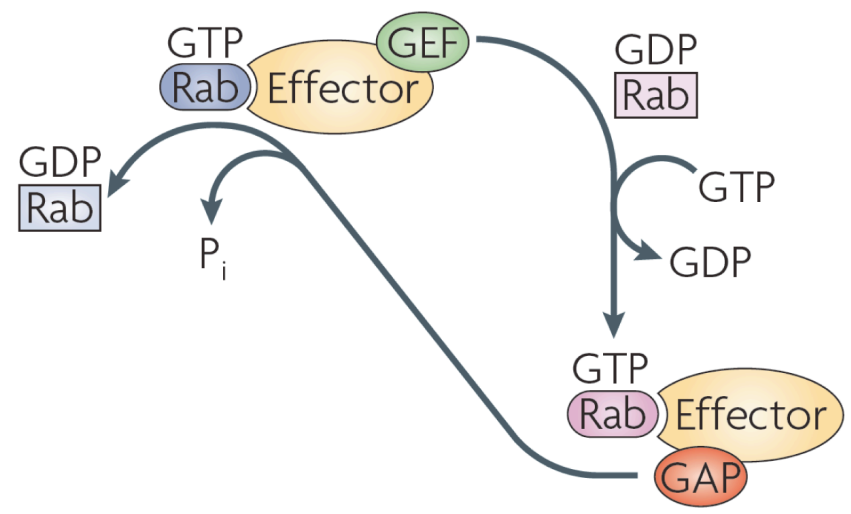

Figure II.13. Model of Rab conversion and exclusion cascade. In a counter Rab exclusion cascade, a downstream Rab recruits the GAP for an upstream Rab to displace it and complete the maturation. The Rab conversion cascade is also depicted, as it is thought that both cascades occur in synchrony. Source: Picture taken from Stenmark, 2009.

\subsection{Cell-type specific functions of Rabs}

Given the large number of Rabs, it was thought that they may be important for cell-type specific functions (Elferink et al., 1992). The first mammalian Rab genes were identified from rat brain and hence termed ras genes from rat brain (Rab) (Touchot et al., 1987). Since then, many more studies have elucidated the role of Rab proteins in the brain. A proteomic analysis of synaptic vesicles revealed a large number of Rabs on SVs (Takamori et al., 2006). Furthermore, Rab3 and Rab27 have been shown to be important for SV exocytosis (Mahoney et al., 2006a; Yu et al., 2008; Pavlos et al., 2010). It appears that the evolution of a nervous system has placed greater demands for regulated membrane trafficking. Since neurons are highly polarized, these cells perhaps require a large number of Rabs to regulate their 
function. Interestingly, there still remain many neuronally expressed Rabs whose functions remain uncharacterized (Elferink et al., 1992; Olkkonen et al., 1994).

\subsection{Redundancy in Rab function}

An important underlying theme in Rab function is redundancy, a strategy employed by the cell allowing multiple proteins to functionally replace each other and to efficiently modify a working mechanism. This was first observed for Rab3. There are four different genes encoding isoforms of Rab3: Rab3A, Rab3B, Rab3C and Rab3D. All four isoforms are highly expressed in the brain (Schlüter et al., 2002). Knockout studies in mice revealed that deletions in a single Rab3 gene resulted in no visible defect in SV exocytosis. Only when all four Rab3 genes are deleted, can a mild decrease in exocytosis be observed (Schlüter et al., 2004). Rab27A and Rab27B, in mice, were also shown to regulate dense granule secretion from blood platelets in a partially redundant manner (Tolmachova et al., 2007). Lastly Rab8 and Rab10 were also shown to cooperate in basolateral sorting of VSV-G (Vesicular stomatitis virus glycoprotein) in MDCK cells. Co-depletion of both Rabs leads to missorting of VSV$\mathrm{G}$ to the apical membrane (Schuck et al., 2007). 


\section{AIMS}

Rab GTPases are the major regulators of membrane trafficking in the cell. Considering that there are over 60 members encoded in the mammalian genome, very little is known about the function of the majority of Rab GTPases. Where are they expressed? How do they all localize with respect to each other? Which effector proteins do they bind to? And how are their functions integrated?

Conducting a family-wide analysis of the Rab family in the mammalian system is a challenge. This arises, not only due to the expanded family size, but also due to the inherent redundancy present in the system. Additionally the generation of genetic tools for such a study would require significant time, before functional analyses could be performed. Therefore, we turned to the model organism Caenorhabditis elegans. C. elegans nematodes have a relatively much smaller Rab family encoding 28 Rab members (Fig. III.1) (Pereira-Leal and Seabra, 2001). Additionally, a major advantage is that deletion mutants for $90 \%$ of the Rab genes are available for analyses. Considering also that $C$. elegans retains major mammalian tissue-types, is transparent for imaging and equipped with versatile genetic tools it was decided to conduct the family-wide characterization of RAB GTPases in $C$. elegans.

The project is divided into 7 parts aimed at analyzing different properties of each C. elegans RAB. (I) Firstly an expression pattern analysis was conducted to understand if RABs are expressed in a cell-type specific manner. (II) Then, a detailed sub-cellular localization analysis was conducted in their respective tissues. (III) As a third step, the Rab deletion mutants were analyzed for insights into function. Since it 


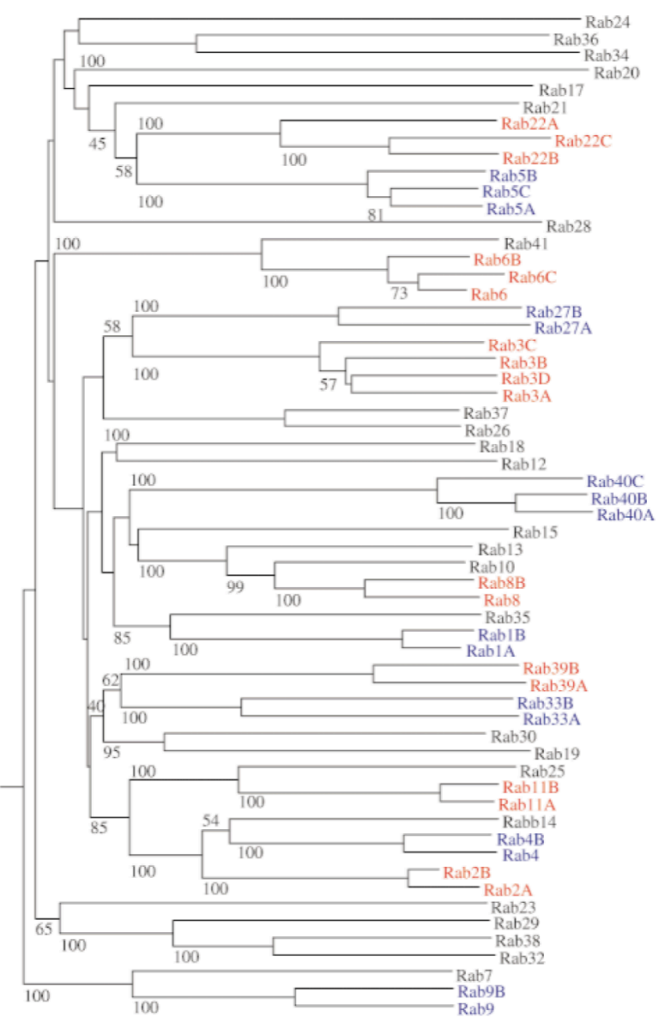

Homo sapiens

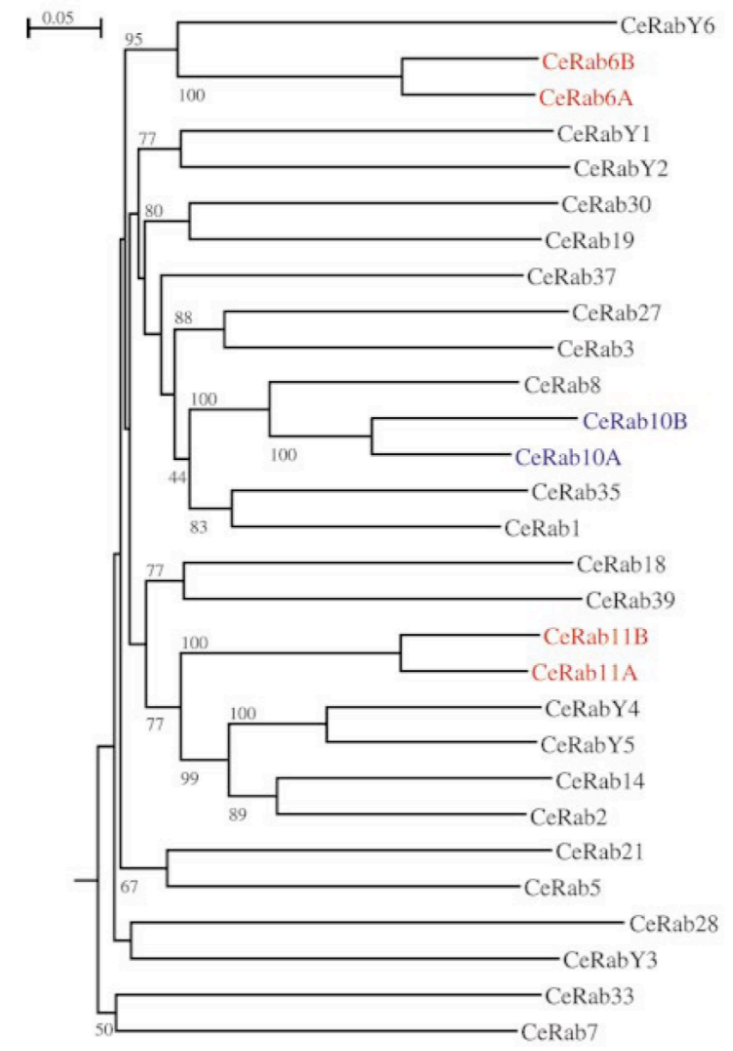

Caenorhabditis elegans

Figure III.1. Comparison of the $\boldsymbol{H}$. sapiens and $\boldsymbol{C}$. elegans Rab families. The human family encodes over 60 members, often with multiple isoforms for a single Rab. This is observed with Rab3, for which there exists 4 isoforms: Rab3A, Rab3B, Rab3C and Rab3D. The nematode Rab family is significantly smaller encoding only 28 members. Often there is only one isoform per Rab and never more than two. Source: Picture taken from Pereira-Leal and Seabra, 2001.

has already been shown that Rabs function redundantly together, we anticipated that this would still hold true in C. elegans despite the smaller Rab family. (IV) Therefore we conducted synthetic RNAi screens to elucidate, which RABs cooperate together. (V) We are also analyzing the biochemical properties of individual RABs by studying their intrinsic rates of GTP hydrolysis and affinities for GTP/GDP (conducted in collaboration with Roger Goody, Max Planck Institute for Molecular Physiology in Dortmund). (VI) As a sixth step we aimed to identify novel RAB effectors using a large scale yeast-2-hybrid approach (conducted in collaboration with Ralf Baumeister, Albert-Ludwigs-University in Freiburg). (VII) As a final step, to understand how Rabs evolved in nematodes and other species, we are conducting a detailed phylogenetic analysis of Rabs over multiple organisms (conducted in collaboration 


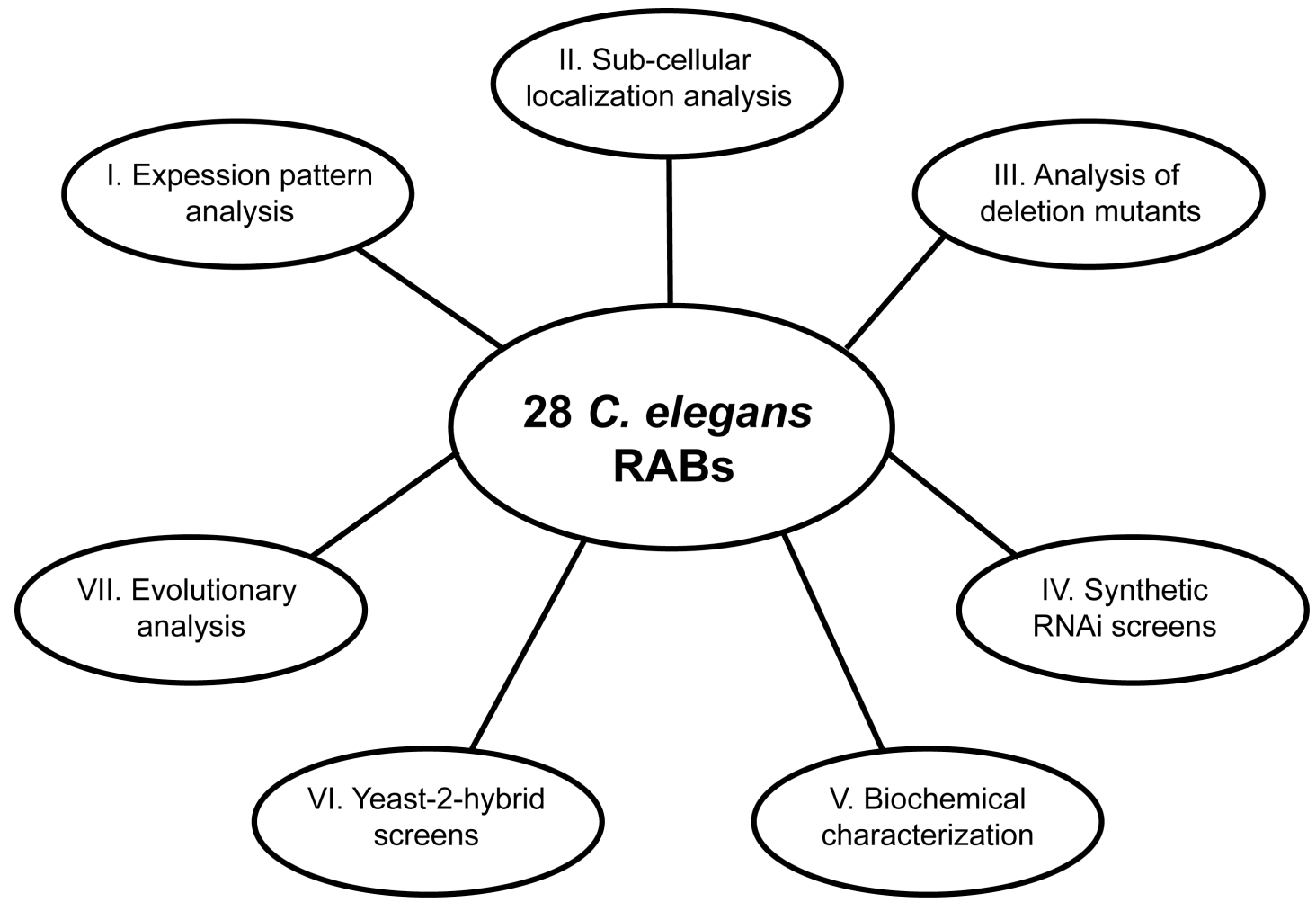

Figure III.2. Schematic representation of project aims. Information about the properties of each C. elegans RAB was collected by an analysis of its expression pattern and sub-cellular localization. This information was then used to analyze the $r a b$ mutants, followed by a synthetic RNAi analysis to determine which RABs function together. In collaboration with other laboratories, we are currently also analyzing the biochemical properties of each RAB, screening through a yeast-2-hybrid approach for novel effectors and investigating how the Rab family evolved in multiple organisms.

with Dirk Fasshauer, University of Lausanne). For the thesis, only data from the parts I - IV are included, as these were the parts that represented most of the author's PhD work. Parts V- VII were mostly initiated through collaborations and are currently ongoing. The aims of the project are summarized in Figure III.2.

Additionally it must be mentioned that experiments for parts I and II were completed solely by the author, part III was done by the author and with help from other members of the lab (Christian Olendrowitz and Marija Sumakovic) and part IV was conducted by the author with assistance from a lab technician (Sabine Koenig). Experiments where the author received help are indicated in the figure legends.

This study represents the first family-wide characterization of Rab GTPases within a model organism including an analysis of mutants. This work will serve as a reference and catalogue for scientists in the field of membrane trafficking. 


\section{RESULTS}

\subsection{Expression pattern analysis of $C$. elegans $\mathrm{RABs}$}

The broad-scale analysis of the $C$. elegans RAB family was initiated by analyzing the expression patterns of each individual rab. For this purpose, a transcriptional promoter::gfp fusion approach was taken. The upstream promoter region of each $r a b$ gene was PCR amplified and cloned by fusing it to green fluorescent protein (GFP). Promoter regions for C. elegans are typically defined as the genomic loci between the start codon of the gene of interest and the stop codon of the upstream gene (Boulin et al., 2006). For this study, most promoter regions cloned were approximately $3 \mathrm{~kb}$ in size, and also incorporated 15-20 base pairs from the start of the $r a b$ gene. A 3'UTR is also necessary for expression. The classical 3'UTR from the unc-54 gene was used. All cloned promoters were injected into wild type animals to analyze GFP expression in all major C. elegans tissues (Fig IV.1, Fig. IV.2).

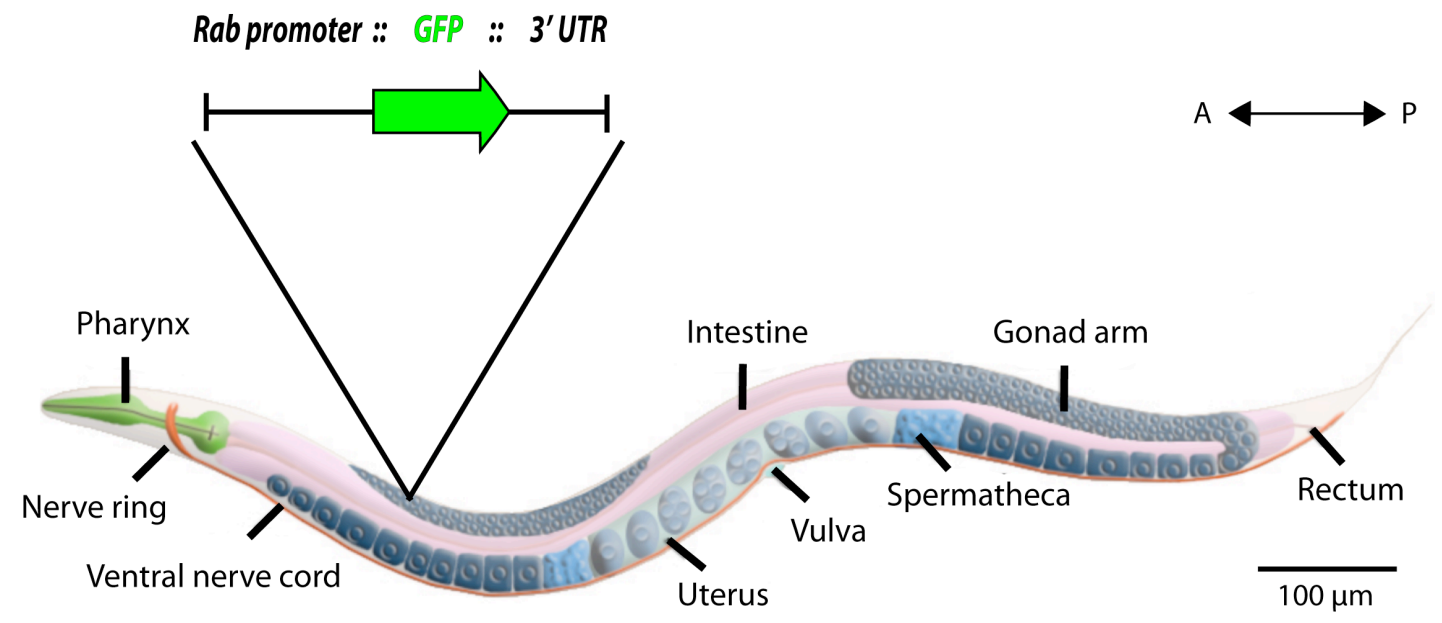

Figure IV.1. Strategy for expression pattern analysis. $r a b$ promoters were cloned and fused to GFP. The classical 3'UTR from the unc-54 gene was used (vector: pPD115.62). C. elegans retains many major tissue types found in vertebrates. The expression of GFP in these tissues was analyzed for each Rab. Source: Picture adapted from Altun and Hall, 2008) 

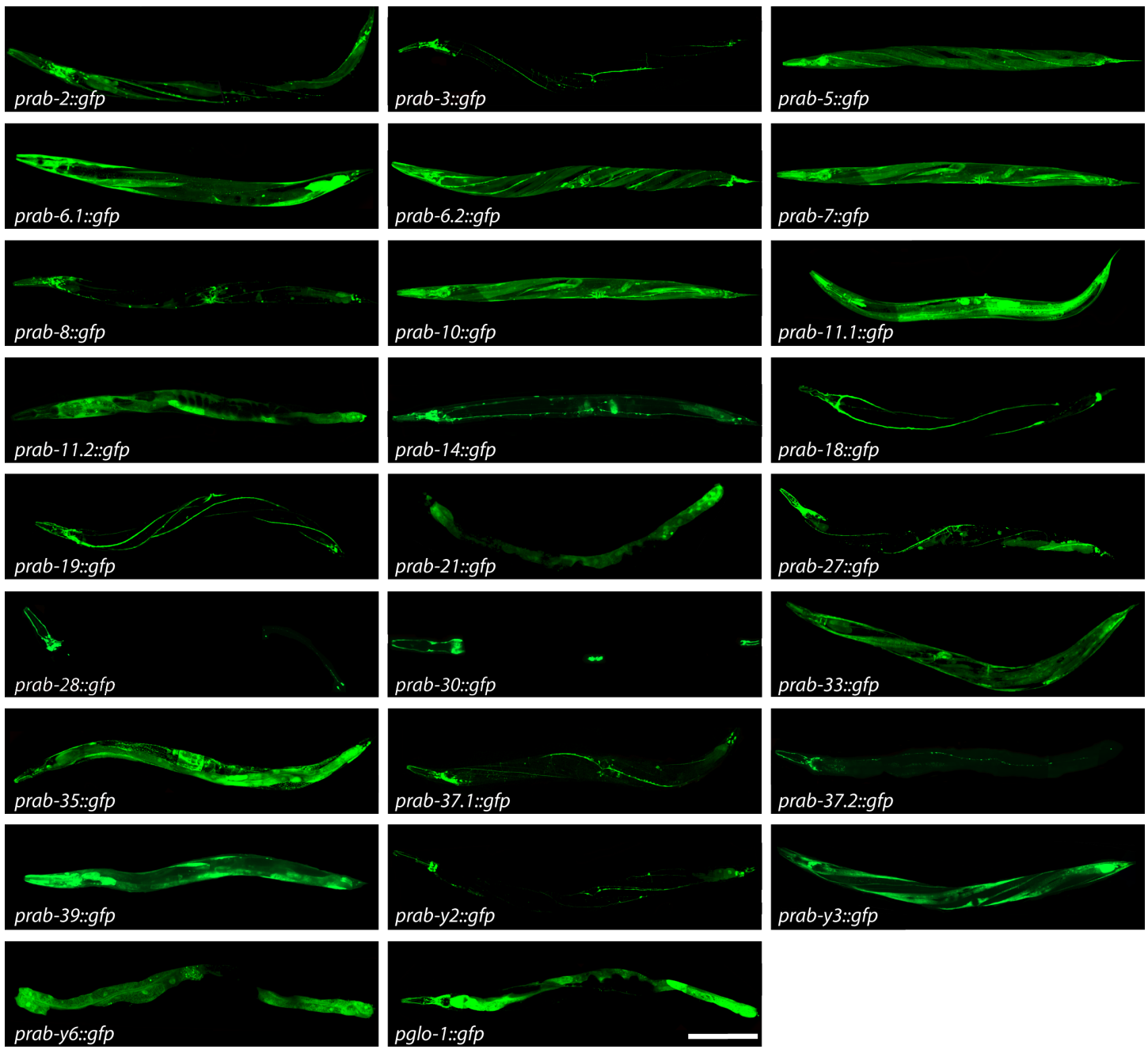

Figure IV.2. Expression patterns of $\boldsymbol{C}$. elegans rabs. Transcriptional fusions of all rab promoters revealed that $C$. elegans rabs are differentially expressed in a tissue-specific manner. All animals are presented on an anterior to posterior axis. The majority of rabs show preference for the nervous system and the intestine in adult animals. A few predicted $r a b$ genes displayed no promoter activity $(r a b-18.1, r a b-y 4$ and $r a b-y 5)$. Additionally, attempts to clone $r a b-1$ and $r a b-y 1$ promoters were unsuccessful. Scale bar represents $200 \mu \mathrm{m}$.

Primary expression pattern analysis revealed that rabs are differentially expressed in C. elegans. Some rabs, such as rab-5, rab-6.1, rab-6.2, rab-8, rab-10 and $r a b-11.1$ were ubiquitously expressed in all tissues. Almost all rabs were expressed in neurons. The second major RAB containing tissue was the intestine. Additionally, some rabs displayed very specific expression patterns. For example, rab-28 expression was restricted to a sub-type of neurons; the ciliated sensory neurons. The expression of $r a b-30$ was observed only in the glial sheath cells of the worm. $r a b-21$ and $r a b-y 6$ were specifically expressed in the intestine. Interestingly $r a b-18, r a b-y 4, r a b-y 5$ promoters did not display any activity. Therefore, from this point onwards $r a b-18.2$ was denoted as $r a b-18, r a b-y 4$ and $r a b-y 5$ were regarded as non-functional pseudo-genes. Attempts to amplify these from a cDNA library also 
failed, further suggesting that they were not expressed. The original number of predicted $r a b$ genes was annotated at 31 . After this expression pattern analysis, it was confirmed that there are a total of $28 \mathrm{rab}$ genes expressed in C. elegans.

All the primary expression pattern data was tabulated to create a list of each $r a b$ matched with the respective tissues in which it is expressed (Table IV.1). It is important to note that the promoters of $r a b-1$ and $r a b-y 1$ could not be amplified by PCR. Information on their expression patterns was available on www.wormbase.org from an ongoing genome wide promoterome project and incorporated into Table IV.1.

Table IV.1. Tabulated representation of expression pattern data

\begin{tabular}{|c|c|c|c|c|c|c|c|c|c|}
\hline & Neurons & Intestine & Glia & Epidermis & Pharynx & Muscle & Vulva & $\mathrm{CC}$ & H-cell \\
\hline$r a b-1^{*}$ & $\bar{X}$ & $\bar{X}$ & $\mathrm{X}$ & $\bar{X}$ & $\bar{X}$ & $X$ & $\bar{X}$ & $\mathrm{X}$ & $\mathrm{X}$ \\
\hline$r a b-2$ & $\mathrm{X}$ & $\mathrm{X}$ & $X$ & $\mathrm{X}$ & $\mathrm{X}$ & $\mathrm{X}$ & $\mathrm{X}$ & $X$ & $\mathrm{X}$ \\
\hline$r a b-3$ & $\mathrm{X}$ & & & & & & & & \\
\hline$r a b-5$ & $X$ & $X$ & $X$ & $X$ & $X$ & $X$ & $X$ & $X$ & $X$ \\
\hline rab-6.1 & $\mathrm{X}$ & $\mathrm{X}$ & $X$ & $\mathrm{X}$ & $\mathrm{X}$ & $X$ & $\mathrm{X}$ & $X$ & $\mathrm{X}$ \\
\hline$r a b-6.2$ & $\mathrm{X}$ & $\mathrm{X}$ & $X$ & $\mathrm{X}$ & $X$ & $X$ & $X$ & X & $X$ \\
\hline$r a b-7$ & $X$ & $X$ & $X$ & $X$ & $X$ & $\mathrm{X}$ & $X$ & $X$ & $X$ \\
\hline rab-8 & $\mathrm{X}$ & $\mathrm{X}$ & $X$ & $X$ & $\mathrm{X}$ & $\mathrm{X}$ & $\mathrm{X}$ & $X$ & $\mathrm{X}$ \\
\hline$r a b-10$ & $X$ & $X$ & $X$ & $X$ & $X$ & $\mathrm{X}$ & $\mathrm{X}$ & $X$ & $X$ \\
\hline$r a b-11.1$ & $X$ & $X$ & $X$ & $X$ & $X$ & $X$ & $X$ & $X$ & $X$ \\
\hline$r a b-11.2$ & & $\mathrm{X}$ & & & & $\mathrm{X}$ & & & \\
\hline$r a b-14$ & $X$ & $X$ & & & & & & $X$ & \\
\hline$r a b-18$ & $X$ & & & & & & & & $X$ \\
\hline$r a b-19$ & $\mathrm{X}$ & & & & & & & & $\mathrm{X}$ \\
\hline$r a b-21$ & & $\mathrm{X}$ & & & & & & & \\
\hline$r a b-27$ & $X$ & $X$ & & & & & & $X$ & \\
\hline$r a b-28$ & $\mathrm{X}$ & & & & & & & & \\
\hline$r a b-30$ & & & $X$ & & & & $\mathrm{X}$ & & \\
\hline$r a b-33$ & $X$ & $X$ & $X$ & $X$ & $X$ & $X$ & $X$ & $X$ & $X$ \\
\hline$r a b-35$ & $\mathrm{X}$ & $\mathrm{X}$ & $\mathrm{X}$ & $\mathrm{X}$ & $\mathrm{X}$ & $\mathrm{X}$ & $\mathrm{X}$ & $X$ & $\mathrm{X}$ \\
\hline$r a b-37.1$ & $X$ & & & & & & & & \\
\hline$r a b-37.2$ & $\mathrm{X}$ & & & & & & & & \\
\hline$r a b-39$ & $\mathrm{X}$ & $\mathrm{X}$ & $\mathrm{X}$ & $X$ & $\mathrm{X}$ & $\mathrm{X}$ & $\mathrm{X}$ & $X$ & $\mathrm{X}$ \\
\hline$|r a b-y|^{*}$ & $X$ & & & & $X$ & & & & \\
\hline$r a b-y 2$ & $\mathrm{X}$ & $\mathrm{X}$ & & & $X$ & & & & \\
\hline$r a b-y 3$ & $X$ & $X$ & & & $X$ & $X$ & & & \\
\hline$r a b-y 6$ & & $X$ & & & & & & & \\
\hline glo-1 & $X$ & $X$ & & & & & & & \\
\hline
\end{tabular}

*Indicates that the promoters for these genes could not be cloned. The expression pattern data was consequently extracted from wormbase (www.wormbase.org)

CC: Coelomocytes and H-cell is the same as the excretory cell. 


\subsection{Sub-cellular localization analysis of $C$. elegans RABs in neurons}

Since the expression pattern analysis revealed that the majority of RABs are expressed in the nervous system, it was decided to analyze the sub-cellular localization of these RABs in neurons. For this purpose, all RABs were cloned with an N-terminal fusion to red fluorescent protein (mCherry/tagRFP) and expressed under a neuron specific promoter ( $r a b-3$ promoter). These constructs were then coinjected with different compartmental markers (Figure IV.3). MANNS II-YFP (Mannosidase II) was used as a marker for the Golgi. GFP-2xFYVE was used as a marker for early endosomes. CTNS-1-YFP was used as a marker for lysosomes. GFP-RAB-6.1 was used as a marker for TGN and endosomes (Eimer et al., 2007; Roggo et al., 2002).

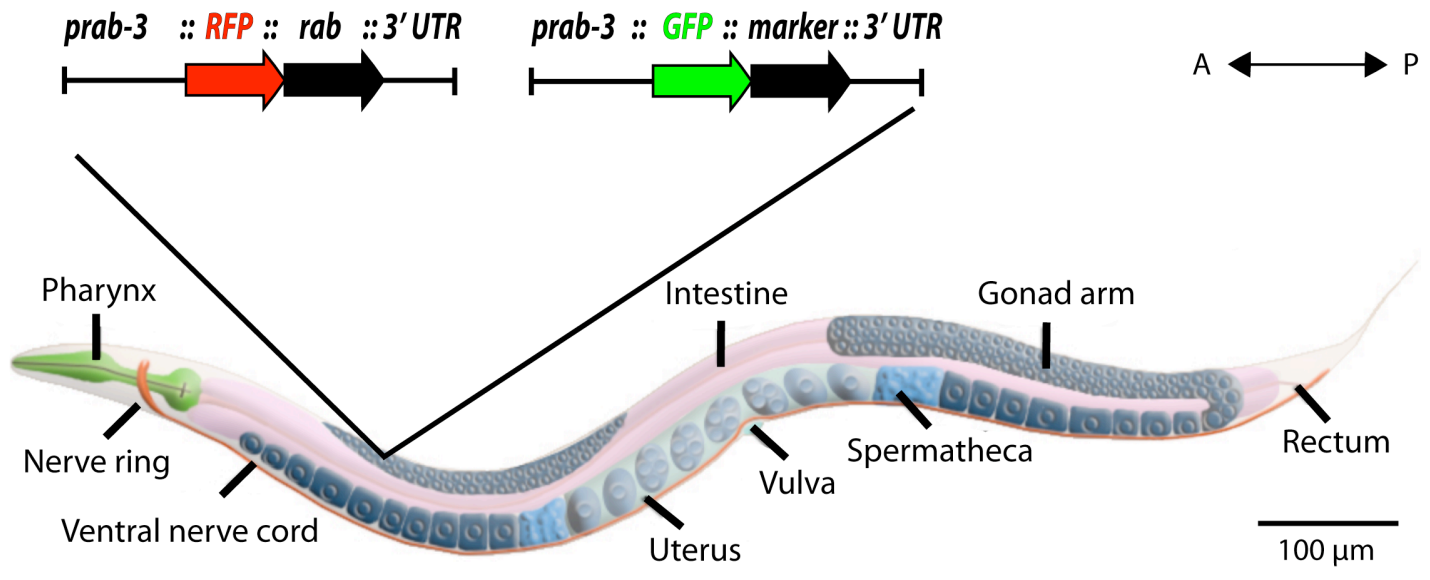

Figure IV.3. Strategy for sub-cellular localization analysis. All rab genes were cloned and Nterminally fused with mCherry/tagRFP. The fusions were expressed in neurons under the $r a b-3$ promoter. Compartmental markers for Golgi, endosomes, and lysosomes were fused to GFP/YFP and co-injected to assess co-localization. Source: Picture adapted from Altun and Hall, 2008

Most neuronally expressed RABs were well localized to the Golgi (Fig. IV.4). RAB-6.1 and RAB-6.2 colocalized with the TGN and endosomes. RAB-8 and RAB10 displayed partial overlap with Golgi and endosomal markers. RAB-14 showed a strong co-localization with RAB-6.1. RAB-35 appeared to localize specifically to neurites and neuronal processes. We are currently testing its localization relative to cytoskeletal markers. RAB-3, RAB-10, RAB-27 and RAB-37 showed strong overlap with synaptic marker proteins (data not shown). RAB-7 and RAB-28 were specific to late endosomes and lysosomes. There were a few RABs, which showed only partial 


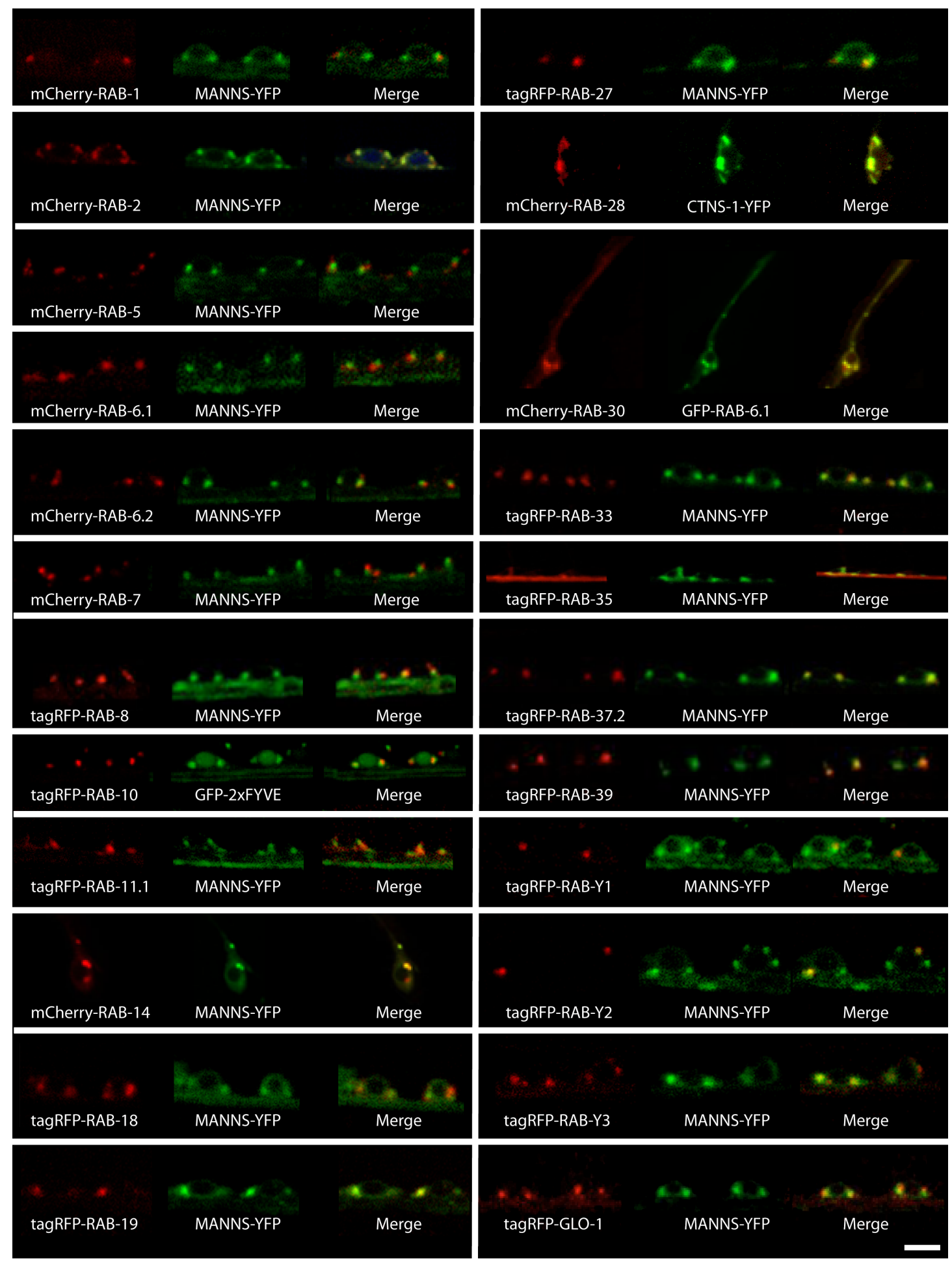

Figure IV.4. Sub-cellular localization of $C$. elegans RABs in nervous system. Each row represents 1-3 neuronal cell bodies, generally imaged from the VNC. Only RAB-30 was imaged in glial cells due to its restricted expression pattern. The $C$. elegans RABs localize to distinct compartments. Many show a pattern of preference for the Golgi. Images for RAB-2 were kindly provided by Marija Sumakovic. Scale bar represents $5 \mu \mathrm{m}$.

colocalization to the Golgi: RAB-1, RAB-18, RAB-Y2 and GLO-1. Additionally the RAB-Y1 and RAB-37.1 showed no colocalization with the Golgi. More markers need to be tested to fine map the localizations of these RABs. 
All primary data from the sub-cellular localization analysis of RABs in the $C$. elegans nervous system was tabulated and is represented in Table IV.2. Please note that additional markers were tested that are not depicted in Fig IV.4, but are described in Table IV.2.

Table IV.2. Tabulated representation of sub-cellular localization data

\begin{tabular}{|c|c|c|}
\hline & Tissue analyzed & Localization \\
\hline RAB-1 & Neurons & Partial Golgi \\
\hline RAB-2 & Neurons & Golgi \\
\hline RAB-3 & Neurons & Golgi/Synapse \\
\hline RAB-5 & Neurons & Endosome/Synapse \\
\hline RAB-6.1 & Neurons & TGN/Endosome \\
\hline RAB-6.2 & Neurons & TGN/Endosome \\
\hline RAB-7 & Neurons & Late Endosome/Synapse \\
\hline RAB-8 & Neurons & Partial Golgi \\
\hline RAB-10 & Neurons & Partial Golgi/Synapse \\
\hline RAB-11.1 & Neurons & Endosome \\
\hline RAB-14 & Neurons & TGN/Endosome \\
\hline RAB-18 & Neurons & Partial Golgi \\
\hline RAB-19 & Neurons & Golgi \\
\hline RAB-27 & Neurons & Golgi/Synapse \\
\hline RAB-28 & Neurons & Lysosome \\
\hline RAB-30 & Glia & Golgi \\
\hline RAB-33 & Neurons & Golgi \\
\hline RAB-35 & Neurons & Partial Golgi/Neurites \\
\hline RAB-37.1 & Neurons & Unknown \\
\hline RAB-37.2 & Neurons & Golgi/Synapse \\
\hline RAB-39 & Neurons & Golgi \\
\hline RAB-Y1 & Neurons & Unknown \\
\hline RAB-Y2 & Neurons & Partial Golgi \\
\hline RAB-Y3 & Neurons & Golgi \\
\hline GLO-1 & Neurons & Partial Golgi \\
\hline
\end{tabular}

To investigate the localizations of $C$. elegans RABs in non-neuronal tissues, we are collaborating with Barth Grant at Rutgers University, New Jersey, USA. The Grant lab is currently analyzing the localization of several RABs in the intestine.

\subsection{Functional analysis of $r a b$ mutants}

The expression pattern data also suggested that most $C$. elegans Rab proteins must function in modulating nervous system function. Since, nematodes display several robust nervous system mediated behaviors it was decided to systematically assay these behaviors with the rab mutants that were available. Figure IV.5 shows 


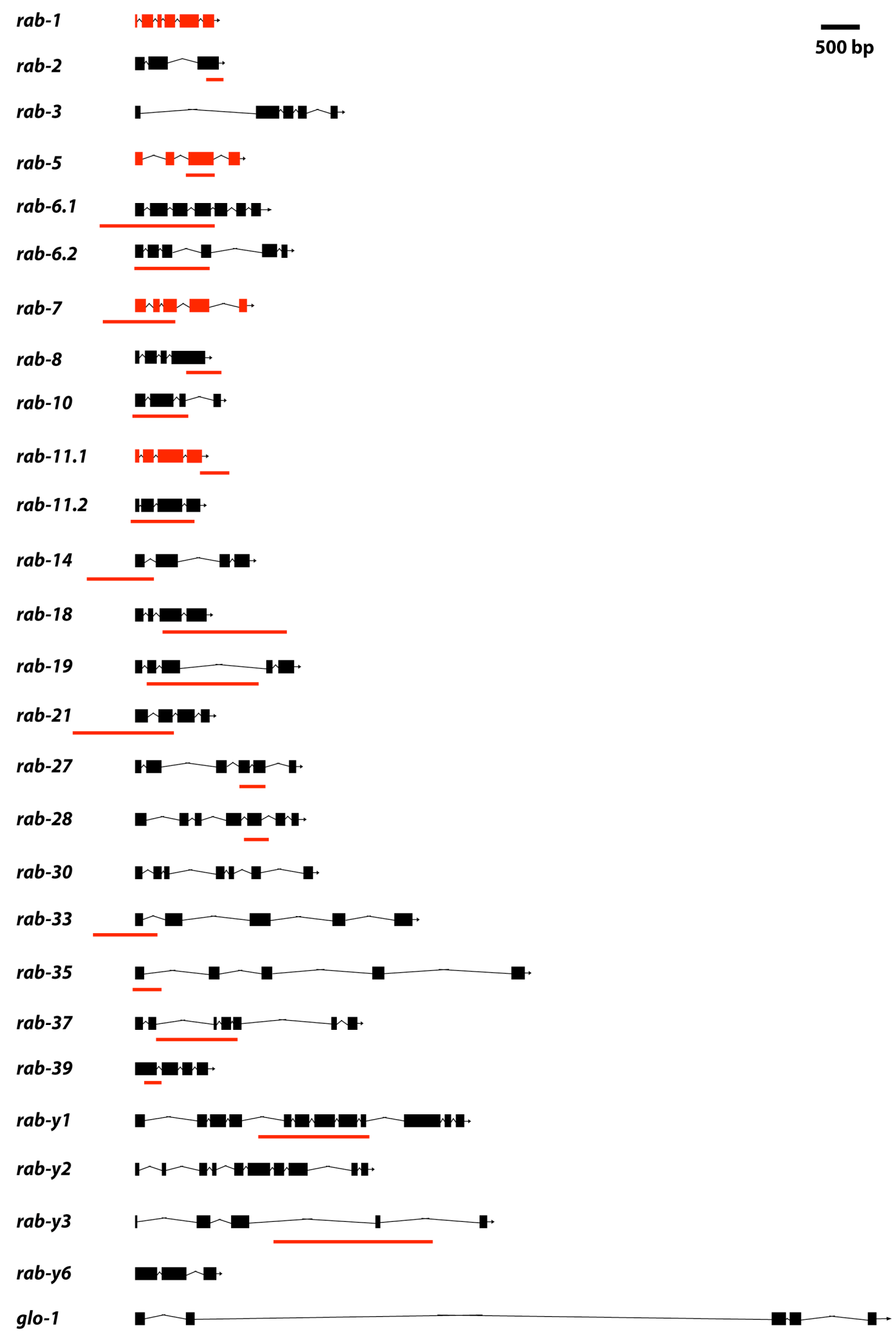

Figure IV.5. Gene structures of all rabs. The gene structures of all $r a b$ genes are depicted. Exons are shown as blocs and introns as connecting lines. Lethal genes are highlighted with red exons. The deletion alleles used in this study are underlined in red below the respective gene. Only mutants with nonsense mutations were available for $r a b-3$ and glo-1. No Mutants for $r a b-30, r a b-y 2$ and $r a b-y 6$ were available. Although $r a b-7$ is lethal, it is a maternal effect lethality, which allows one generation to be assayed. Sizes of the deletions and official allele names are described in the appendix. 
the gene structures of all the $C$. elegans rab genes, along with a depiction of which mutants were available for analysis and which mutants were lethal.

\subsubsection{Movement analysis}

C. elegans movement is a complex behavior, which is coordinated by the neuro-muscular system. The body wall muscles of $C$. elegans run in eight quadrants along the nerve cords of the animal. Four quadrants flank the dorsal nerve cord and four quadrants flank the ventral nerve cord (Fig. IV.6). There are 6 major classes of motor neurons, which are responsible for locomotion: VA, VB, VD, DA, DB and DD

\section{A}

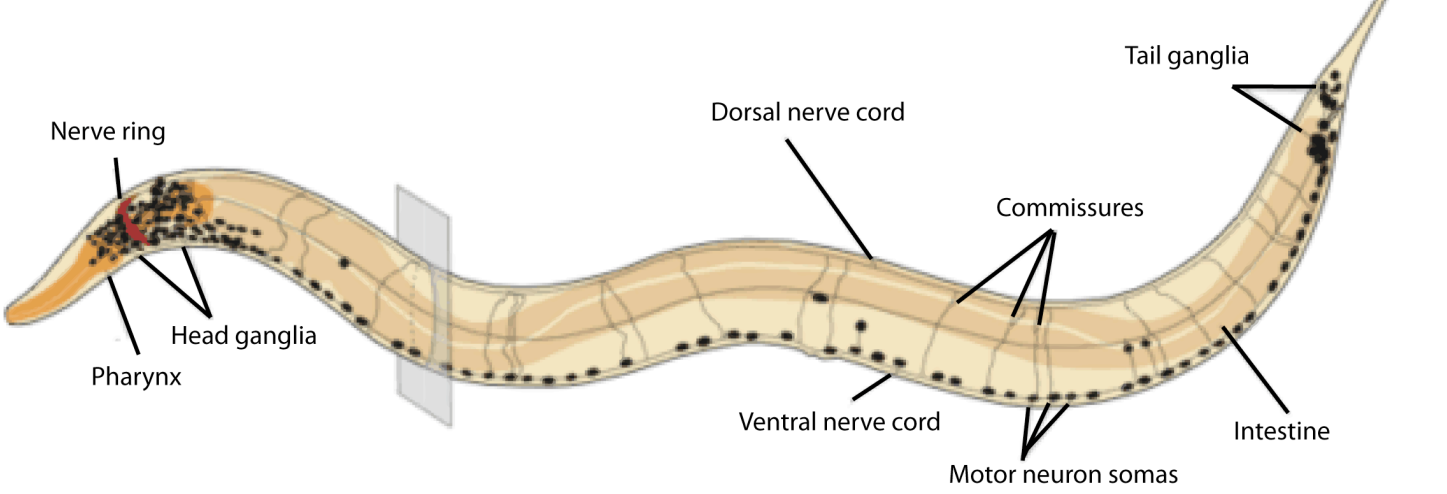

B

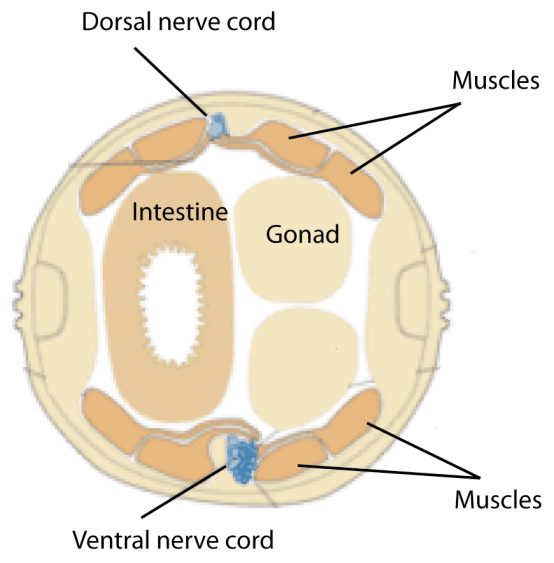

C

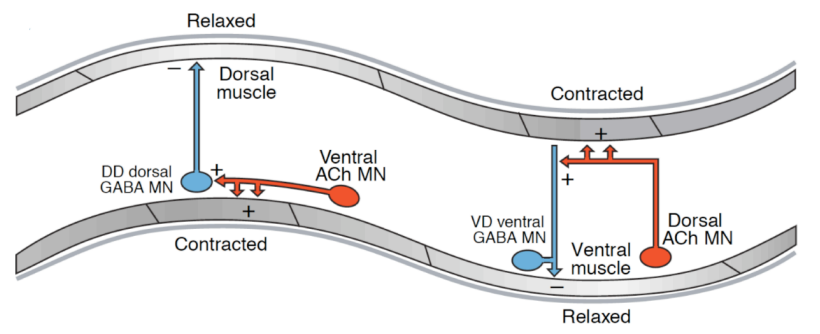

Figure IV.6. The neuro-muscular system of C. elegans. A. C. elegans has a ventral and dorsal nerve cord, which run through the length of the animal's body. B. A cross-section of the animal reveals that the body wall muscles important for movement run as four quadrants flanking each nerve cord C. All motor neurons are located in the VNC. VA, VB and VD neurons form neuro-muscular junctions (NMJs) with the ventral muscles and DA, DB and DD neurons form NMJs with the dorsal muscles. Worms move in a sinusoidal fashion. This is achieved by stimulation of muscles on one side of the animal, along with simultaneous inhibition of the muscles on the contralateral side (Schuske et al., 2004). Source: Pictures for A. and B. were provided by Stefan Eimer. Image for C was adapted from Schuske et al., 2004. 
(von Stetina et al., 2006). The cell bodies of all these neurons are located in the ventral nerve cord (VNC). VA, VB, and VD form synapses with adjacent muscles in the $\mathrm{VNC}$, whereas DA, DB and DD sent contralateral commissures to form synapses at the dorsal nerve cord (DNC) (Riddle et al., 1997).

The VA, VB, DA and DB motor neurons are cholinergic, whereas the VD and DD motor neurons are $\gamma$-Aminobutyric acid (GABA)ergic. Nematodes move in a sinusoidal fashion. This movement is mediated by the simultaneous contraction and relaxation of the ventral and dorsal muscles. The VB motor neurons activate muscles on the ventral side, and in parallel activate the DD motor neurons, which relax the muscles on the dorsal side. On the other hand, the DB motor neurons activate muscles on the dorsal side, and in parallel activate the VD motor neurons to relax the muscles on the ventral side (Figure IV.6) (von Stetina et al., 2006).

To assess if the rab mutants were capable of coordinated movement, we measured their movement rates. This was done by counting the number of sinusoidal body bends per minute (Figure IV.7). Wild type animals displayed a rate of $23.47 \pm$ 0.72 body bends per minute. $r a b-2$ mutants were severely uncoordinated and had an average rate of $11.98 \pm 0.46$ body bends per minute. $r a b-2$ was previously identified in a screen for uncoordinated mutants and is hence also called unc-108 (Brenner, 1974). All other rab mutants displayed normal rates of locomotion.

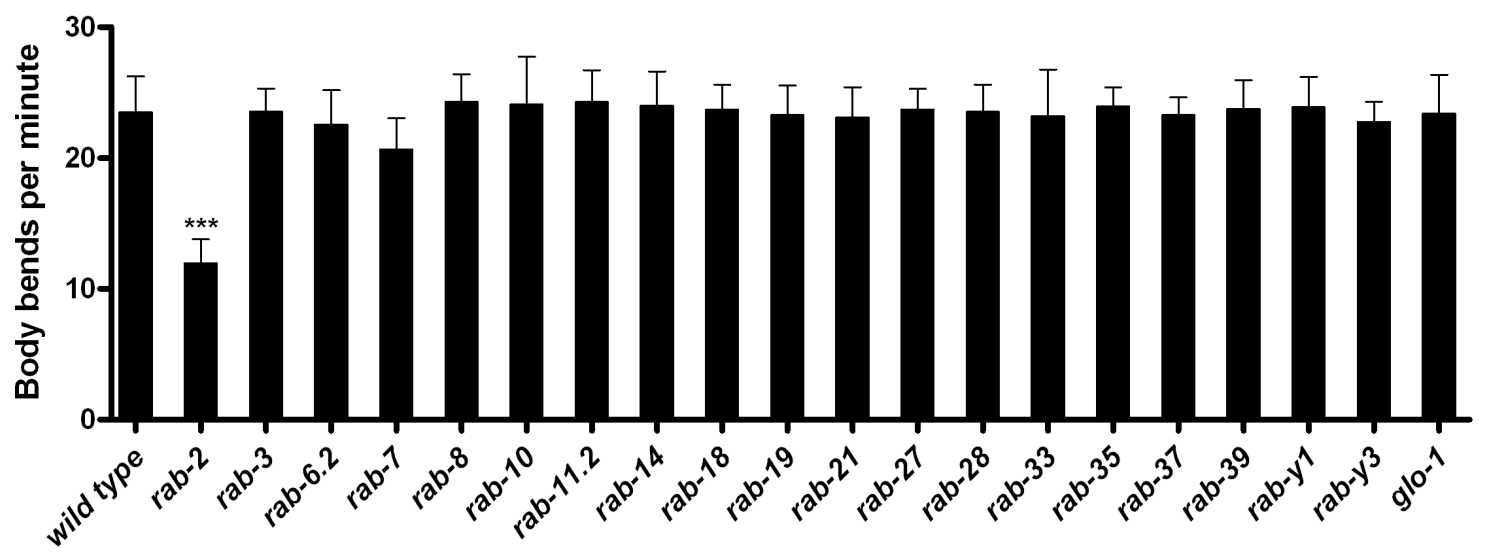

Figure IV.7. Movement analysis of $r \boldsymbol{r a b}$ mutants. The movement of all $\mathrm{rab}$ mutants was analyzed by measuring their body bends per minute. $r a b-2$ mutants are strongly uncoordinated. All other $r a b$ mutants display normal locomotion. At least $\mathrm{N}=10$ animals were tested per strain. Error bars $=\mathrm{SEM}$ (***, P $<0.005$; ANOVA with Bonferroni post test). Source: This experiment was conducted by the author, Marija Sumakovic and Christian Olendrowitz. 


\subsubsection{Defecation motor program analysis}

Another stereotypic behavior of $C$. elegans regulated by the nervous system is defecation. The defecation motor program of $C$. elegans occurs through a series of defined stages: The first stage involves the contraction of the posterior body muscles (pBoc), where the intestinal contents are pushed to the head. This stage is then followed by an anterior body contraction (aBoc), where the intestinal contents are sent back to the tail. The last step is the enteric muscle contraction (Emc), where the anus is opened to allow contents to be released (Fig. IV.8). This cycle occurs approximately every 45-50 seconds, when animals are kept on food. Cycle length is thought to be controlled by the intestine. Two neurons are important for Emc: DVB and AVL (Schuske et al., 2004). These neurons form atypical excitatory GABAergic synapses onto the anal depressor muscles. Ablation of AVL and/or DVB neurons abolishes the Emc and leads to accumulation of feces in the intestine (McIntire et al., 1993).

To investigate if RABs are involved in regulating the defecation motor program, we tested the rab mutants for defects in defecation (Fig. IV.9). Two different parameters were measured: the length of the defecation cycle (time between subsequent pBocs) and the expulsion efficiency (ratio of expulsions to pBocs). Wild type animals had a cycle length of $46.13 \pm 0.80 \mathrm{sec}$ and a $100 \%$ expulsion efficiency. $r a b-6.2, r a b-7, r a b-8, r a b-18$ and $r a b-35$ mutants displayed over $10 \%$ increases in

A

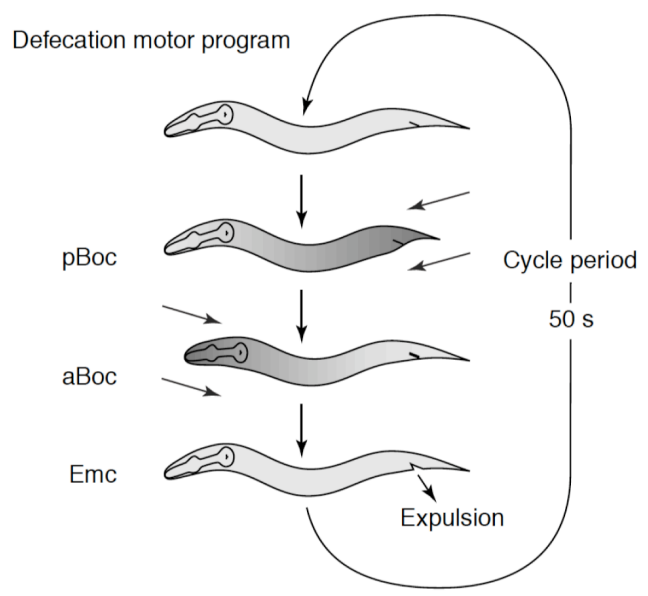

B

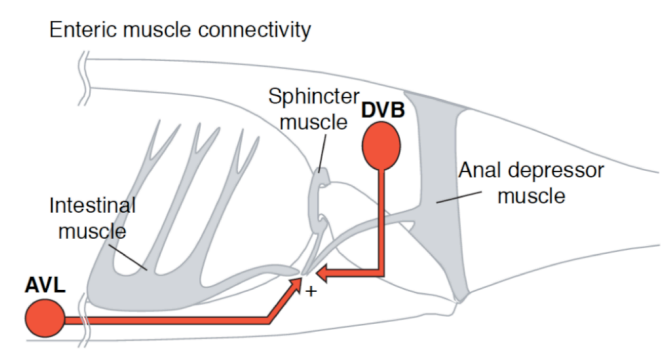

Figure IV.8. The defecation motor program. A. C. elegans defecation occurs in three steps: pBoc, aBoc and Emc. The cycle repeats itself approximately every 50 seconds when animals are on food $\mathbf{B}$. Two neurons, AVL and DVB control the Emc by synapsing onto the anal depressor muscles. Source: Pictures taken from Schuske et al. 2004. 


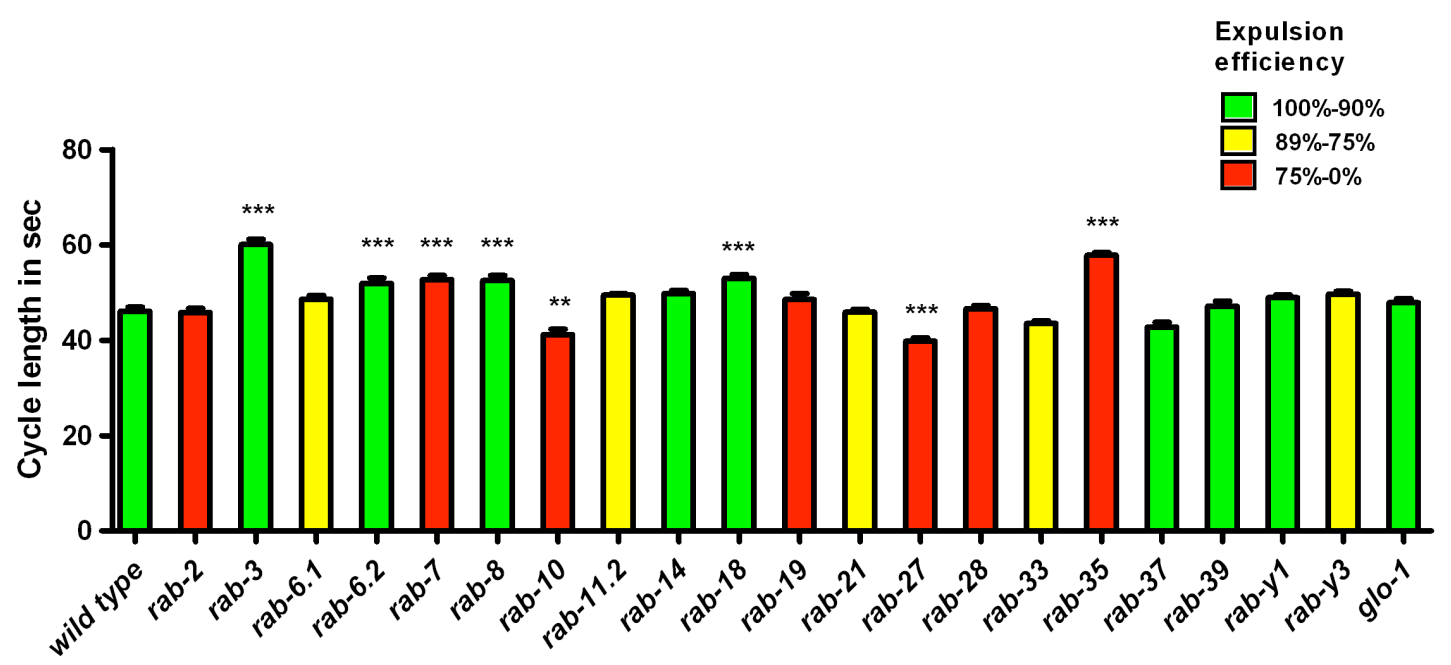

Figure IV.9. Defecation analysis of $\boldsymbol{r} \boldsymbol{a} \boldsymbol{b}$ mutants. Defecation was analyzed by measuring the average cycle length (the time taken between pBocs). Additionally the expulsion efficiency for each mutant was measured. Green indicates no defect in expulsion efficiency, yellow indicates a mild defect and red indicates a severe defect. At least $\mathrm{N}=10$ animals were per strain were tested. Error bars $=\mathrm{SEM}$ (***, $\mathrm{P}<0.005 ; * *, \mathrm{P}<0.01$; ANOVA with Bonferroni post test). Source: This experiment was conducted by the author, Marija Sumakovic and Christian Olendrowitz.

cycle length compared to wild type animals. $r a b-10$ and $r a b-27$ mutants displayed over $10 \%$ decreases in cycle length. Many rab mutants displayed defects in expulsion efficiency. rab-27 mutants displayed the strongest defect in expulsion with an efficiency of $0.02 \pm 0.01 \%$. Other severely affected mutants were rab-2 $(63.76 \pm 0.07$ \%), rab-7 (58.89 $\pm 0.11 \%), r a b-10(54.35 \pm 0.09 \%), r a b-19(55.72 \pm 0.07 \%), r a b-$ $28(51.38 \pm 0.05 \%)$ and $r a b-35(69.82 \pm 0.03 \%)$.

\subsubsection{Egg-laying analysis}

Another nervous system mediated behavior is egg-laying. Newly fertilized eggs remain in the uterus for 2-3 hours before being released from the vulva. The egglaying behavior is mediated by a pair of motor neurons called the hermaphrodite specific neurons (HSNs). HSNs form serotonergic synapses with the vulval muscles. Ablation of these cells leads to defects in the frequency of egg-laying. Since these animals can still lay eggs, it is thought that they also co-release a secondary neurotransmitter (Schafer, 2005). In addition, VC motor neurons of the VNC are also important for the regulation of egg-laying (Waggoner et al., 1998).

Eggs can be categorized into three developmental stages: 0-8 cell stage, 8 cell to comma stage and postcomma stage. In wild type animals, the majority of laid eggs are in the 8-cell to comma stage. Egg-laying defective animals retain their eggs longer in the uterus and therefore lay more eggs in the postcomma stage. To investigate if 


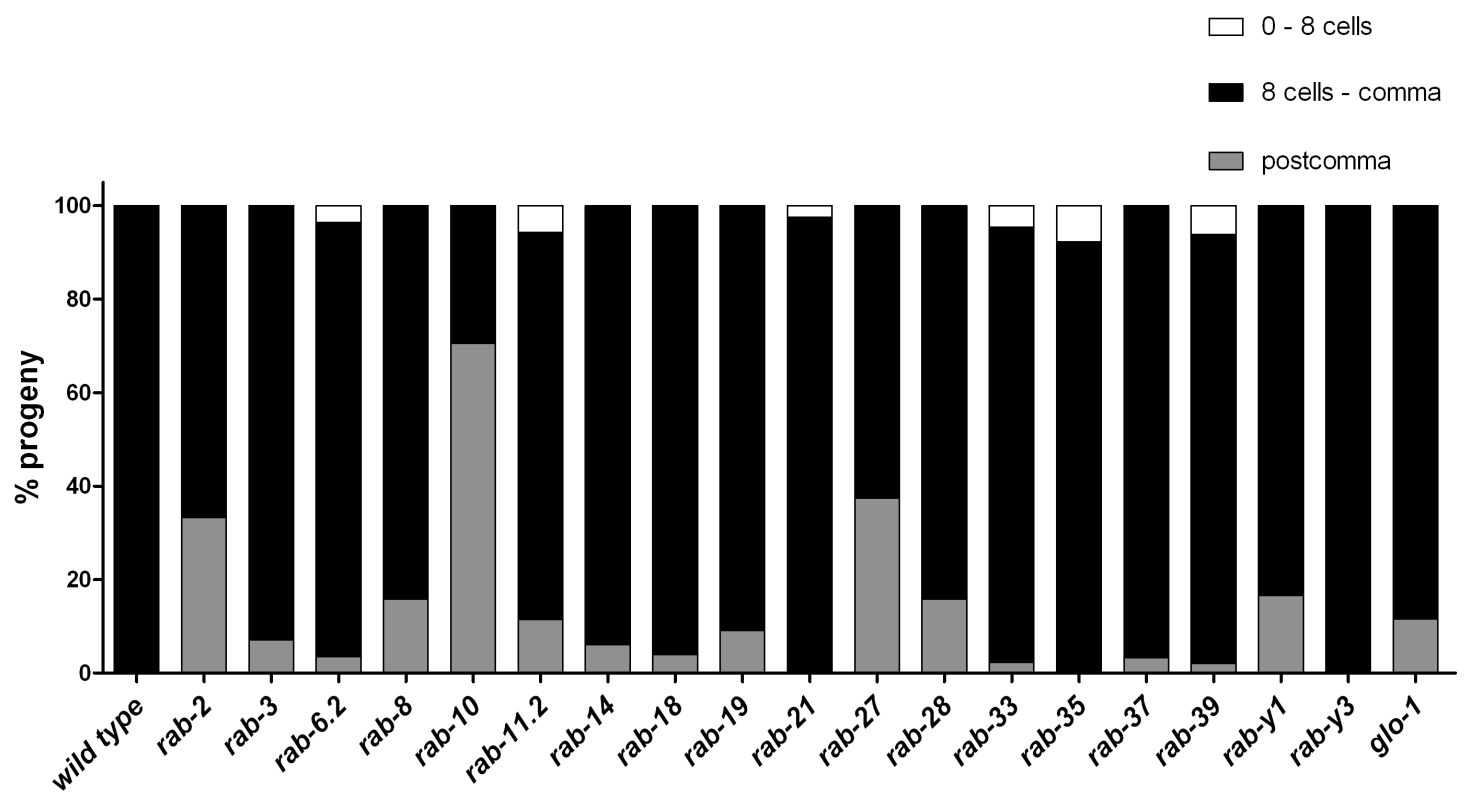

Figure IV.10. Egg-laying analysis of $\boldsymbol{r a b}$ mutants. To assess egg-laying behavior, the eggs were divided into three developmental stages: $0-8$ cell stage, 8 cell-comma stage and postcomma stage. In wild type animals the majority of laid eggs are in the 8 cell-comma stage. Some $r a b$ mutants displayed severe defects in egg-laying by laying over $30 \%$ of their eggs in the postcomma stage. $\mathrm{N}=20$ animals per strain were tested. Source: This experiment was conducted by the author and Marija Sumakovic.

RABs are important in serotonergic neurons to modulate egg-laying, the distribution of eggs in these three stages was analyzed (Fig IV.10). rab-2, rab-10 and rab-27 animals displayed the strongest egg-laying defects. rab-2 mutants laid $33.33 \%$, rab10 mutants laid $70.59 \%$ and rab-27 mutants laid $37.50 \%$ of their eggs in the postcomma stage. $r a b-8, r a b-28, r a b-y 1$ and glo-1 displayed milder egg-laying defects by retaining $10-20 \%$ of their eggs in the postcomma stage. All other rab mutants appeared to display normal egg-laying behavior.

\subsection{Pharmacological analysis of synaptic transmission}

It has been previously shown that $r a b-3$ mutants are important for tethering of SVs at neuronal synapses (Nonet et al., 1997). In order to identify if other neuronally expressed RABs are involved in regulating synaptic transmission, we made use of an aldicarb-based pharmacological assay.

\subsubsection{Aldicarb sensitivity}

Aldicarb is an inhibitor of acetylcholinesterase (AChE), which breaks down acetylcholine (ACh) in the synaptic cleft. Aldicarb exposure creates a build-up of $\mathrm{ACh}$ leading to hyper-stimulation of postsynaptic ACh receptors at the neuro- 
muscular junction (NMJ). This ultimately leads to hyper-contraction of muscles and death of animals (Fig IV.11) (Mahoney et al., 2006b).

The $r a b$ mutants were analyzed for their responses to aldicarb. Animals were exposed to $2 \mathrm{mM}$ aldicarb and then observed after 90 minutes. The percentage of paralyzed worms in the population was measured. After exposure, $39.81 \pm 3.34 \%$ of wild type animals were paralyzed. $r a b-3, r a b-14$ and $r a b-27$ animals were significantly resistant to aldicarb with only $13.00 \pm 3.51 \%, 10.00 \pm 5.77 \%$ and 6.53 $\pm 3.85 \%$ animals paralyzed respectively. $r a b-2, r a b-6.2, r a b-8, r a b-35$ and $r a b-y 1$

A

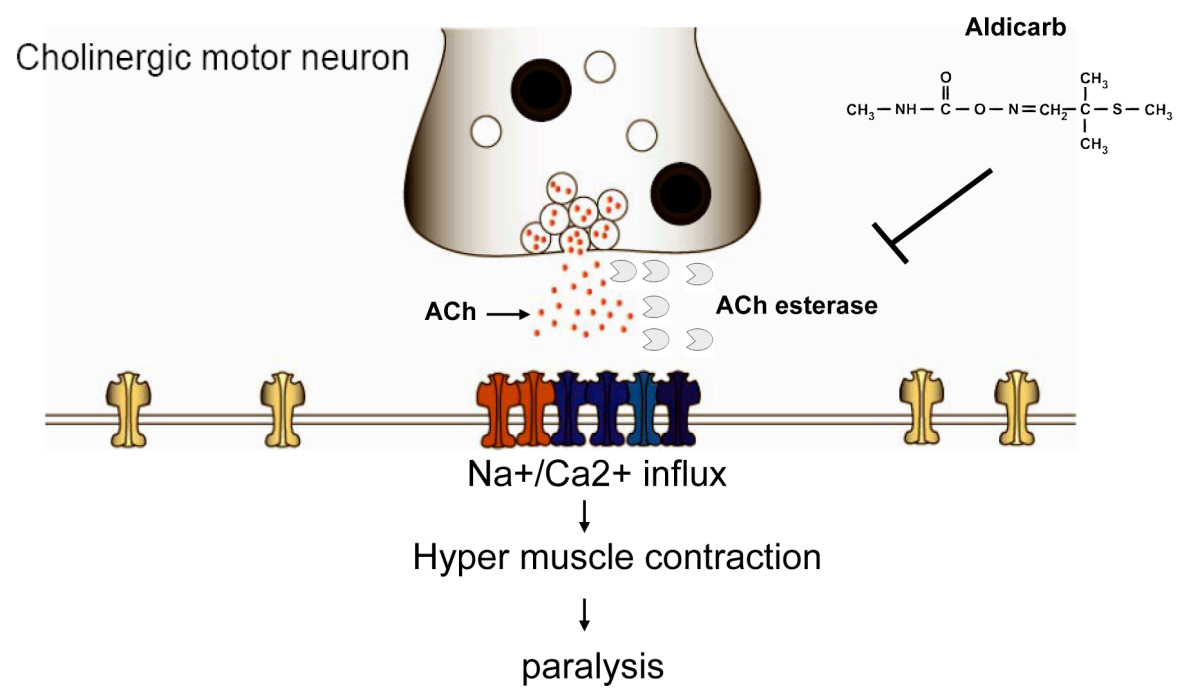

\section{B}

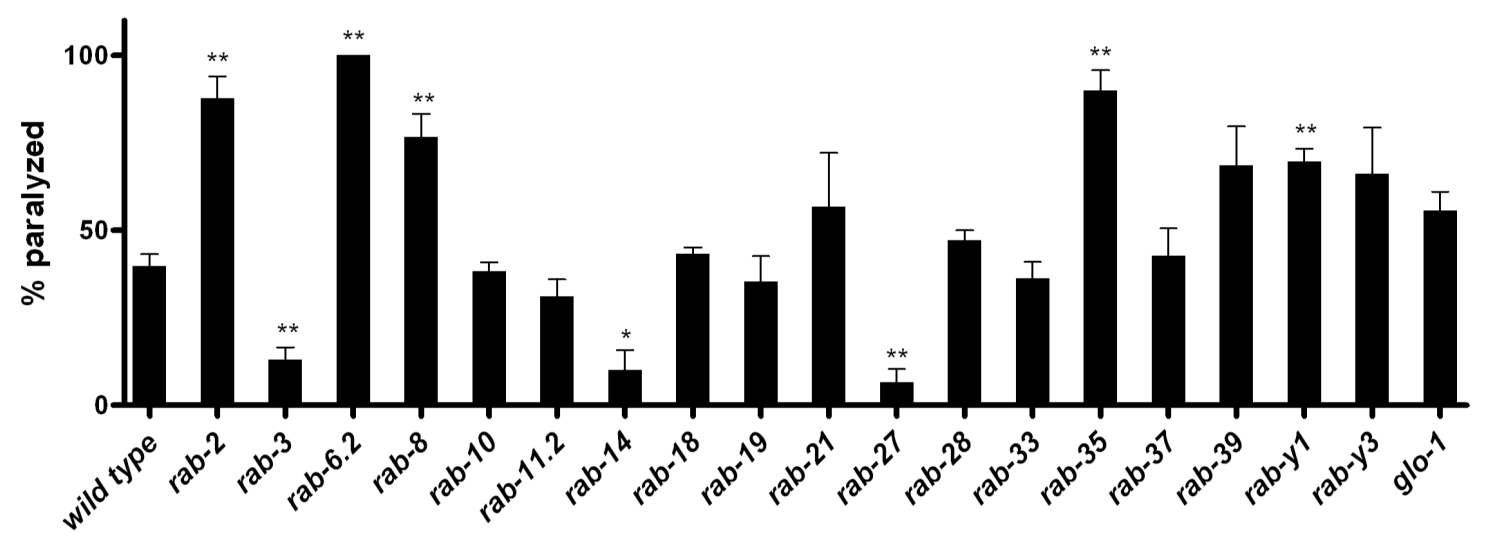

Figure IV.11. Analysis of synaptic transmission through responses to aldicarb. A. Aldicarb is an inhibitor of AChesterase. C. elegans animals, which are exposed to aldicarb, accumulate acetylcholine at the synaptic cleft of NMJs eventually leading to hyperstimulation of ACh receptors and subsequent death by hyper-contraction (Mahoney et al., 2006b). B. Percent paralysis of the rab mutants to $2 \mathrm{mM}$ aldicarb after 90 minutes of exposure was measured. Several $r a b$ mutants were resistant to aldicarb, while others were hypersensitive. $\mathrm{N}=30$ animals per strain were tested. Error bars $=\mathrm{SEM}(* *, \mathrm{P}<$ $0.01 ; *, \mathrm{P}<0.05$ Student's t test; all strains were compared with wild type). Source: This experiment was done by the author and Marija Sumakovic. Picture A. was kindly provided by Marija Sumakovic. 
were hypersensitive to aldicarb with $87.78 \pm 6.12 \%, 100.00 \pm 0.00 \%, 76.67 \pm 6.67$ $\%, 90.00 \pm 5.77 \%$ and $69.64 \pm 3.71 \%$ animals paralyzed respectively (Fig. IV.11)

\subsubsection{Levamisole sensitivity}

The results of aldicarb sensitivity assays indicated that there were several $r a b$ mutants with defects in synaptic transmission. In order to elucidate whether these defects were a consequence of a perturbed postsynapse, the rab mutants were tested for sensitivity to levamisole. Levamisole is an agonist of a subgroup of nicotinic ACh receptors (nAChRs) at NMJs. Upon exposure to levamisole, animals hyper-contract and die (Fig.IV.12) (Lewis et al., 1980). An animal's response to levamisole is largely

A
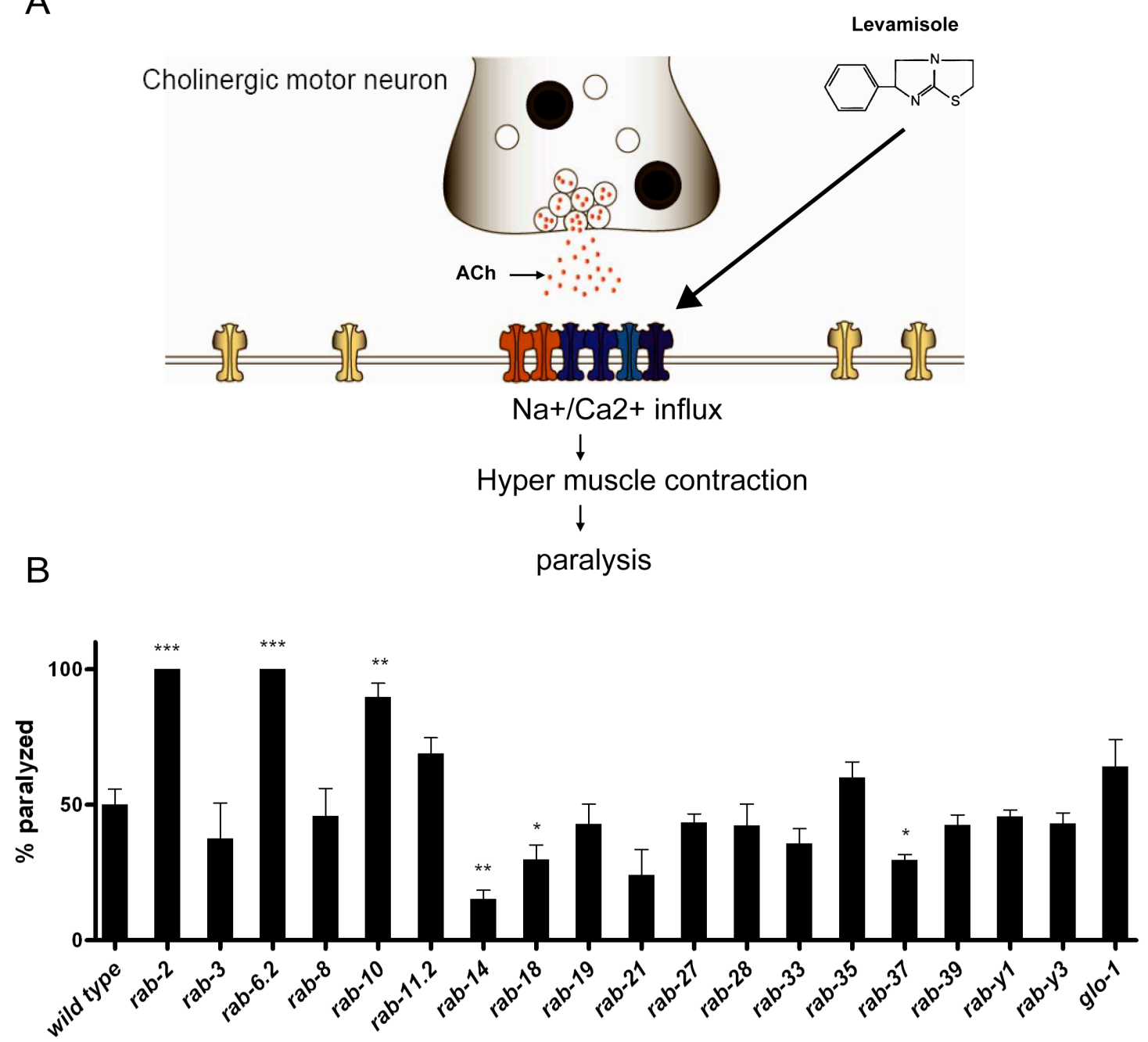

Figure IV.12. Analysis of synaptic transmission through responses to levamisole. A. Levamisole is an agonist of ACh receptors. C. elegans animals, which are exposed to levamisole, undergo hyperactivation of ACh receptors and subsequent death by hyper-contraction (Lewis et al., 1980). B. Percent paralysis of the $r a b$ mutants to $1 \mathrm{mM}$ levamisole after 80 minutes of exposure was measured. Several $r a b$ mutants were resistant to levamisole, while others were hypersensitive. $\mathrm{N}=30$ animals per strain were tested. Error bars $=$ SEM (***, P $<0.005$; **, P $<0.05$; *, P $<0.01$; Student's t test; all strains were compared with wild type). Source: This experiment was done by the author and Marija Sumakovic. Picture A. was kindly provided by Marija Sumakovic. 
dependent on the number, integrity and clustering of postsynaptic ACh receptors (Eimer et al., 2007; Gally et al., 2004). The effect of levamisole on the $r a b$ mutants was analyzed. Animals were exposed to $1 \mathrm{mM}$ levamisole and then observed after 80 minutes. The percentage of paralyzed worms in the population was measured. Levamisole causes $50 \pm 5.77 \%$ of wild type animals to paralyze. $r a b-14, r a b-18$ and rab-37 mutants were resistant to levamisole with only $15.25 \pm 3.23 \%, 29.83 \pm 5.23$ $\%$ and $29.73 \pm 1.84 \%$ of animals paralyzed respectively. rab-2, rab-6.2 and rab-10 were hypersensitive to levamisole with $100.00 \pm 0.00,100.00 \pm 0.00$ and $89.68 \pm 5.20$ $\%$ animals paralyzed respectively (Fig. IV.12).

\subsection{Analysis of ciliated sensory neurons in $r a b$ mutants}

Among the 302 neurons present in C. elegans, a significant number (60) are ciliated sensory neurons dedicated to sensing the external environment (Heiman and Shaham, 2007). Since the RABs are expressed in the nervous system and are important regulators of membrane trafficking, we reasoned that they may regulate either the formation of these ciliated sensory neurons or the function of these neurons.

\subsubsection{DiI staining of amphids and phasmids}

To assess the integrity of the chemosensory neurons, we took advantage of DiI. DiI is a lipophilic dye that fills specifically the amphid sensory neurons in the head and also the phasmid sensory neurons in the tail. The mechanism of dye uptake is not clearly understood, but it is thought to enter via the endings of the ciliated sensory neurons, which are open to the environment. Figure IV.13 shows the anatomical location of the amphids and phasmids (Tong and Bürglin, 2010).

All the rab mutants were stained with DiI and their amphids and phasmids neurons were imaged (Fig. IV.14). $d y f-11$ was used as a control because these

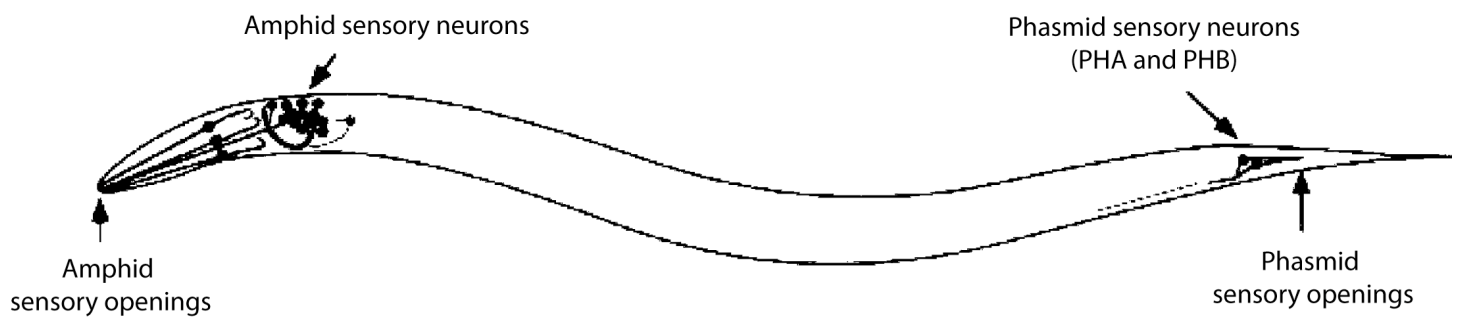

Figure IV.13. Anatomic depiction of $\boldsymbol{C}$. elegans chemosensory neurons. The amphid neurons are located in the head and the phasmid neurons are located in the tail of the worm. Both sets of neurons are readily stained by DiI, which migrates up the sensory openings. Source: Picture taken and adapted from Bargmann, 2006. 
A
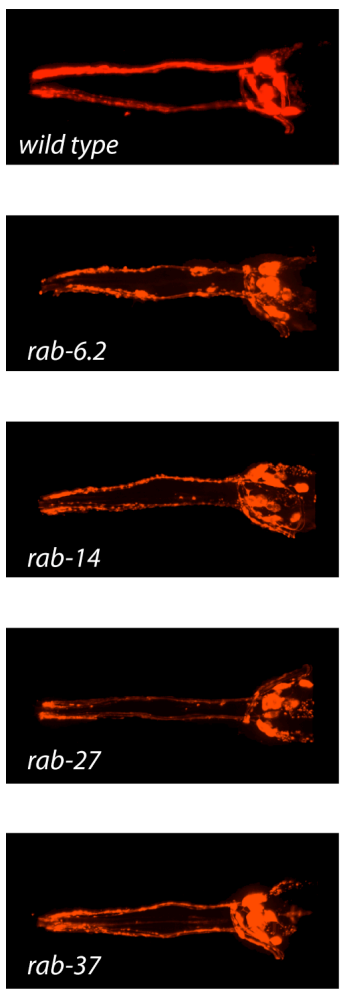

B
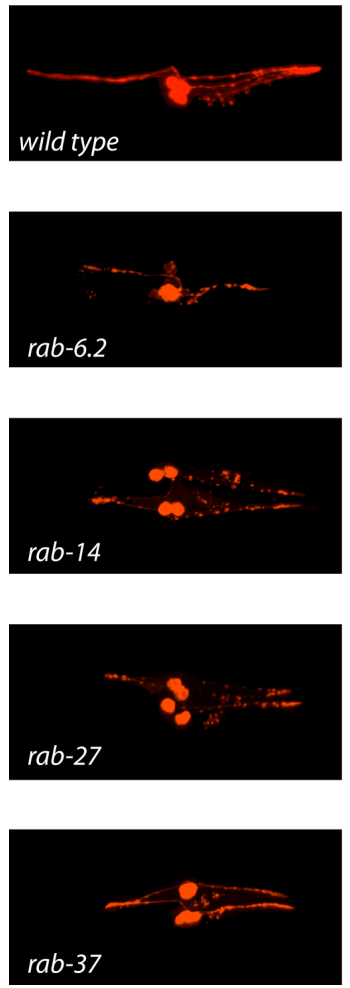

Amphid Neurons
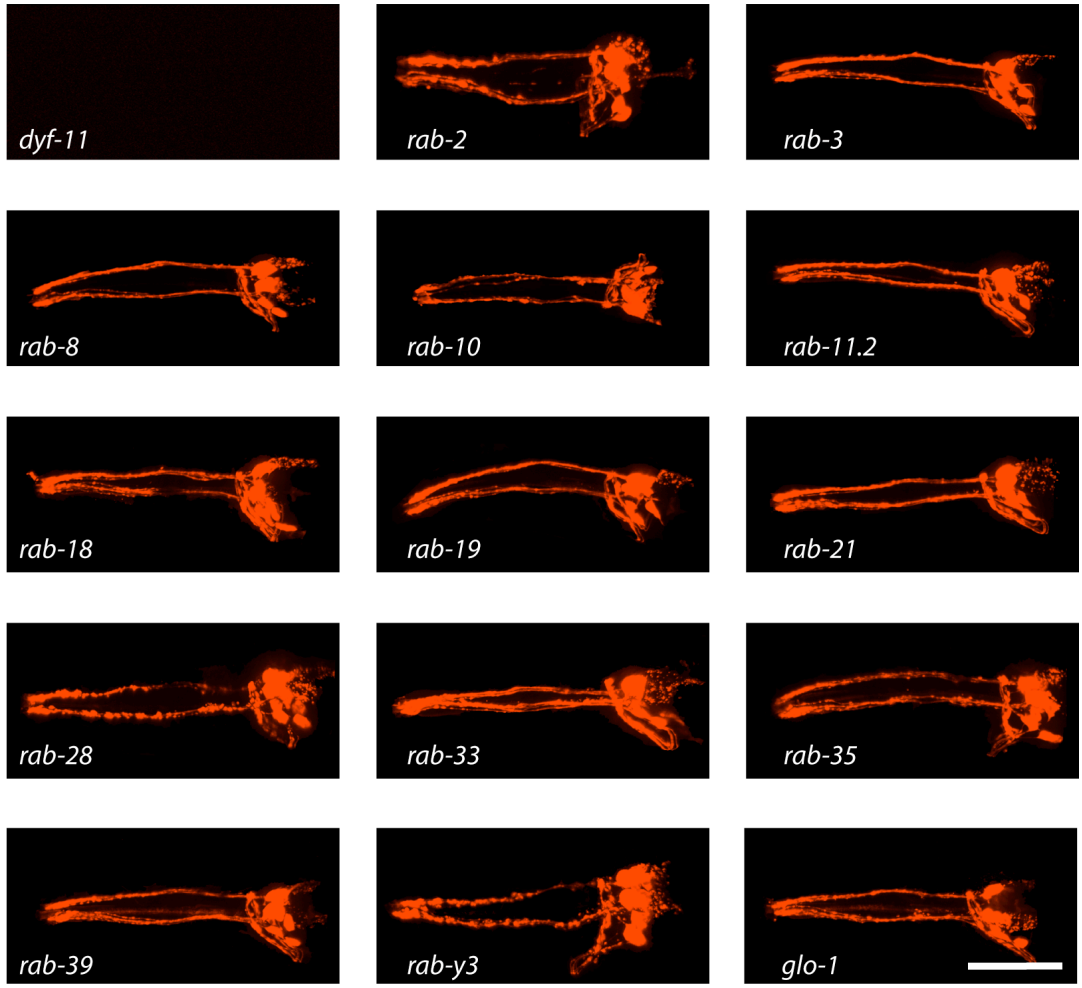

Phasmid Neurons
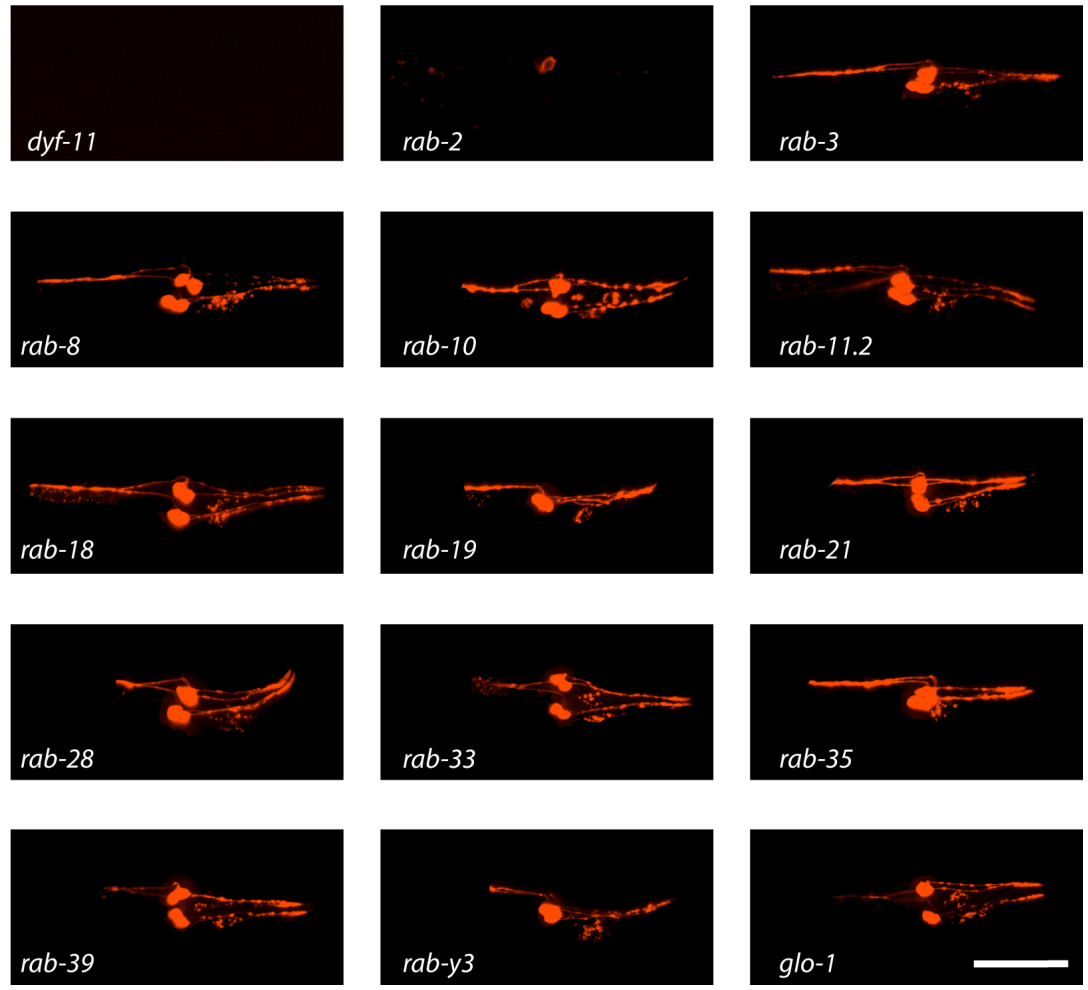

Figure IV.14. DiI staining of $\boldsymbol{r a b}$ mutants. A. DiI staining of the amphid sensory neurons revealed that the morphology of these neurons and their ability to uptake external dyes was intact in all $r a b$ mutants. Bar represents $60 \mu \mathrm{m}$. B. DiI staining of the phasmid sensory neurons revealed that the morphology of these tail neurons was largely unaffected in $r a b$ mutants. Interestingly, only $r a b-2$ mutants appeared to have a strong defect in phasmid dye uptake. Bar represents $60 \mu \mathrm{m}$. 
mutants have a severe failure to uptake DiI (Bacaj et al., 2007). Interestingly, the rab2 mutants had a defect in the uptake of DiI in the phasmid sensory neurons. Aside from $r a b-2$ all other $r a b$ mutants did not display any obvious abnormalities in dye filling.

\subsubsection{Analysis of amphid neuron function}

Despite normal morphology, it is still possible that these neurons are impaired in their function. Therefore, we tested the responses of the amphid neurons to chemical substances. C. elegans displays attraction and avoidance responses to a variety of substances. Among these, copper creates a robust avoidance response through the ASH sensory neuron and isoamyl alcohol creates a robust attraction response through the AWC sensory neuron (Bargmann, 2006). A qualitative assay was set up to test these two responses. Unseeded plates with a line of $150 \mathrm{mM} \mathrm{CuSO}_{4}$ drawn through the middle were prepared. Strains of interest were then spotted to one half of the plate and a drop of isoamylalcohol (1:10) was spotted onto the other half of the plate (Fig. IV.15) (Wicks, 2000).

A

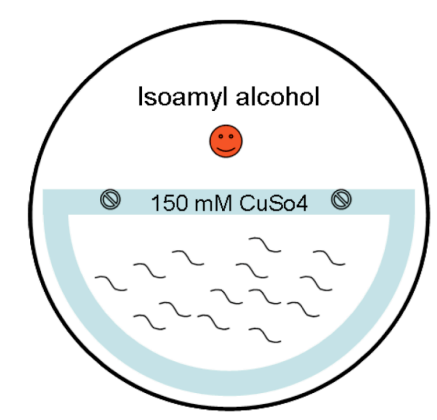

$\mathrm{B}$

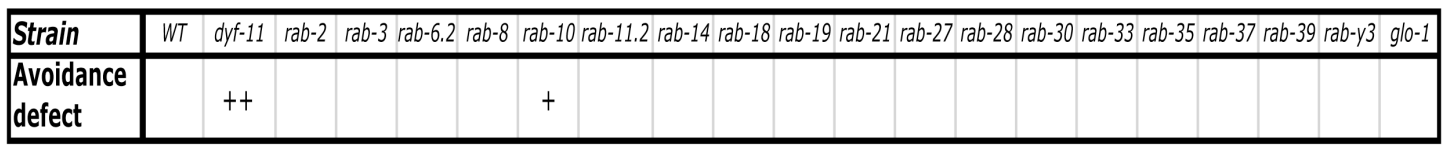

Figure IV.15. Responses of $r a b$ mutants to copper and isoamyl alcohol. A. An attraction/avoidance assay was set up to chemosensory responses of the rab mutants. Plates lined with $150 \mathrm{mM} \mathrm{CuSO}_{4}$ were prepared. Animals were placed on one half of the plate and a drop of isoamyl alcohol (1:10) was placed on the opposite side. After 45 minutes, the animals that crossed the copper line were qualitatively assessed. B. All Rab mutants were tested with this assay. They displayed mostly normal sensory responses to copper and isoamyl alcohol. rab-10 mutants displayed a mild defect in avoiding copper. ++ Indicates at least $40 \%$ of the population crossed the copper line and + indicates between 10 $20 \%$ of population crossed the copper line. Source: Picture was originally prepared by Alejandro Mendoza (lab rotation student). 
The number of animals, which crossed the line of copper, was then assessed qualitatively. All rab mutants were normally attracted to isoamyl alcohol and avoided copper. rab-10 mutants displayed a mild defect in sensing copper.

\subsection{Synthetic RNAi analysis of rab mutants}

Strikingly, most functional assays revealed that $r a b$ mutants are healthy and have fairly mild phenotypes. We reasoned that this is most likely due to redundancy in RAB function. To understand, which RABs overlap in their function a synthetic RNAi screen was conducted. For this purpose, all rab mutants were crossed into an RNAi hypersensitive strain (either eri-1 or rrf-3: to increase RNAi efficiency) and then co-depleted by RNAi with the remaining 27 Rabs. Initially the $r r f-3$ strain was used. However, due to the sickness in this background, the healthier eri-1 strain was used. Furthermore, RNAi potency appeared stronger with eri-l as judged by the recapitulation of known phenotypes. Synthetic phenotypes were then scored independently by two observers. All synthetic interactions are displayed in Fig IV.16. A large variety of interactions were observed including synthetic lethality, uncoordinated behavior, egg-laying defects, sterility etc. For example $r a b-8$ and $r a b$ 10 displayed reciprocal synthetic lethality. $r a b-6.1$ and $r a b-6.2$ were also observed to be synthetically lethal. $r a b-3$ and $r a b-21$ were also synthetically lethal, as were $r a b$ 19 and $r a b-21$. Furthermore, $r a b-35$ was uncoordinated when co-depleted with $r a b-7$. This was further verified by analysis of the double mutants (data not shown). The $r a b-3$; $r a b-27$ double mutant displayed synthetic uncoordinated behavior with $r a b-37$. Interestingly, among all the mutants that were screened, rab-2 mutants showed the largest number of genetic interactions with other rabs. 


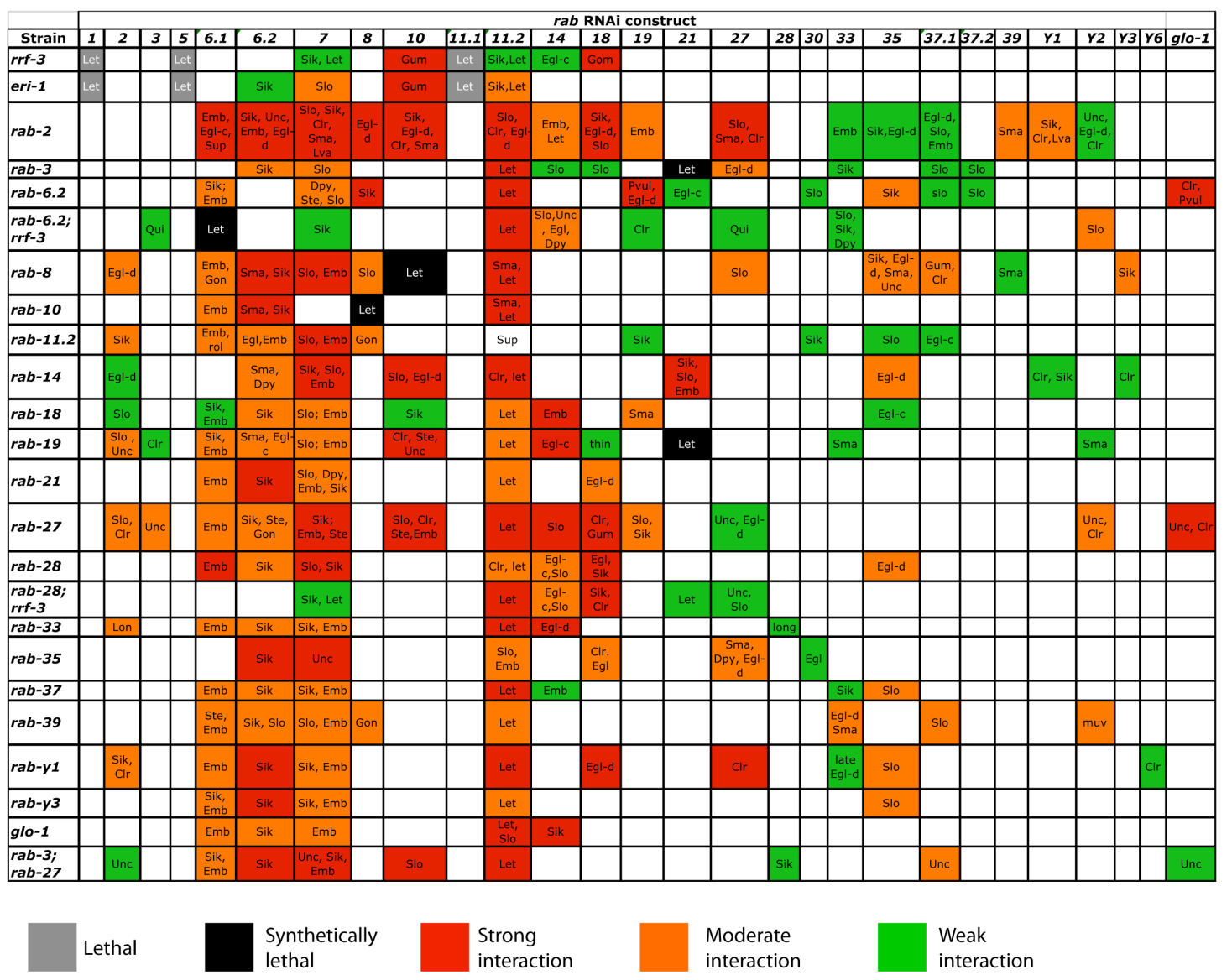

Clr : clear body

Egl-d : egg-laying defective

Gon : gonad defect

Lon : long body size

Qui : quick growing

Sma : small body size

Unc : uncoordinated
Dpy : dumpy

Emb : embryonic lethal

Gum : gut morphology abnormal

Muv : Multi vulva

Sik : sick

Ste : sterile
Egl-c : egg-laying constitutive Gom : gut out morphology

Let : lethal

Pvul : protruding vulva

Slo : slow growing

Thin : Thin

Figure IV.16. Synthetic RNAi screen with rab mutants. Each $r a b$ mutant was crossed into either the eri-1 or $r r f-3$ RNAi hypersensitive background. Initially the $r r f-3$ background was used, but because these animals were relatively unhealthy, we chose to use the healthier eri- 1 background. Furthermore the potency of RNAi, as judged by specific phenotypes, was stronger in the eri-1 background. The majority of $r a b$ mutants are in the eri-1 background, unless otherwise indicated. Each rab mutant was co-depleted with the remaining 28 rabs via feeding RNAi. Additionally a $r a b-3$; $r a b-27$ double mutant was also screened. Two independent observers scored phenotypes. All phenotypes are described by their three letter acronyms, which are explained in the legend. 


\section{DISCUSSION}

\subsection{The $C$. elegans genome encodes 28 differentially expressed rabs}

Rabs are the largest family of small GTPases. With over 60 members encoded in the human genome, they are involved in multiple steps of membrane trafficking. Despite, the large family size, relatively little is known about these proteins. In order to gain insights on $r a b$ function we exploited the model organism C. elegans. Being a genetically tractable organism, with a short generation time, it provided several advantages in contrast to other model organisms. Furthermore, mutants for $90 \%$ of the rabs were available for analysis.

In $C$. elegans, originally it was predicted that there are $31 \mathrm{rab}$ open reading frames expressed (www.wormbase.org). This study was initiated through a systematic analysis of the expression patterns of each of these 31 genes. By analyzing the promoter activity of these genes, it was determined that there are only 28 actively expressed $r a b$ genes in C. elegans. $r a b-18.1, r a b-y 4$ and $r a b-y 5$ did not reveal any expression and appear to be pseudogenes. The expression of these genes in other developmental stages also revealed no expression, nor could these genes be amplified out of a cDNA library. Interestingly, $r a b-y 4$ and $r a b-y 5$ have high similarity to $r a b-2$ suggesting that they are possibly recent duplications, which have not become active. Alternatively, it is also possible that these genes are expressed in very low levels, or that longer promoter constructs incorporating additional regulatory elements are necessary to observe expression. An RT-PCR experiment to measure mRNA levels of $r a b-y 4$ and $r a b-y 5$ would help to detect low-level expression.

An important finding from the expression pattern analysis was that although some rabs were ubiquitously expressed, the majority was expressed in a tissue specific manner. When comparing with the mutants available, it was observed that 
most of the ubiquitously expressed $r a b s$ ( $r a b-1, r a b-5, r a b-6.1, r a b-7$ and $r a b-11.1$ ) were lethal when depleted suggesting that these have a more general role in membrane trafficking. Furthermore, the orthologues of these rabs have been shown in the mammalian system to be essential regulators of trafficking (Nuoffer et al., 1994; Stenmark et al., 1994; Martinez et al., 1994; Feng et al., 1995; Ullrich et al., 1996). The nervous system expressed the highest number of RABs in C. elegans. Due to the highly polarized nature of neurons, it is likely that they have a greater demand for regulated membrane trafficking than other cell-types. In line with our results, an alternative approach to study neuronally expressed genes in C. elegans via microarray profiling also identified many RABs (von Stetina et al., 2007). Furthermore studies in H.sapiens and D. melanogaster, have also showed that many Rabs are tissue-specific and enriched in the nervous system (Takamori et al., 2006; Pavlos et al., 2010; Elferink et al., 1992; Olkkonen et al., 1994; Zhang et al., 2007).

Among the neuronally expressed $r a b s, r a b-3, r a b-27$ and $r a b-37$ were the most specific. Interestingly, when looking at the C. elegans rab phylogenetic tree, these RABs fall into the functional group III by virtue of their similar structures (Pereira-Leal and Seabra, 2001). This suggests that these three Rabs coordinate together in regulating neuronal membrane trafficking. Interestingly, it has already been shown that two of these Rabs: RAB-3 and RAB-27 function together in SV exocytosis in C. elegans (Mahoney et al., 2006a).

The expression patterns of $r a b-28$ and $r a b-30$ were particularly interesting because they were restricted to just a few cells. $r a b-28$ was identified to be expressed only in a subset of neurons, the ciliated sensory neurons (amphids and phasmids). rab-30 was found to be expressed only in the glial sheeth cells which ensheath the amphid and phasmid neurons. These expression patterns suggest that these RABs play a role in the formation of sensory organs or in the trafficking of specific cargos in these cells. Interestingly, $r a b-28$ mutants did not display any obvious defects in sensory neuron function. However, preliminary data suggests that, when RAB-28 is co-depleted with RAB-Y3 or RAB-Y6, a defect in avoiding copper was identified (data not shown). Copper avoidance is mediated by the alpha subunit of a G-protein coupled receptor (GPCR), GPA-3, which is specifically expressed by the ASH chemosensory neuron (Bargmann, 2006). It is possible that RAB-28, RAB-Y3 and RAB-Y6 may be involved in the transport of the GPCR involved in copper sensation. Unfortunately, a rab-30 mutant was not available for analysis. 


\subsection{Multiple RABs localize to the Golgi in neurons}

An important task to understanding RAB function is to identify which compartments they localize to. Since many of the C. elegans RABs were expressed in neurons, we decided to systematically analyze their sub-cellular localizations in these cells. Results revealed that RABs localize to distinct compartments in neurons. For example RAB-1 and RAB-2 localized to the Golgi, RAB-6.1, RAB-6.2 and RAB14 localized to the TGN-endosomal interface, RAB-5 localized to endosomes/synapses, RAB-3, RAB-27, RAB-37 localized to the Golgi/synapses and RAB-28 localized to lysosomes. Despite the varied localization patterns, surprisingly a lot of RABs also showed partial or full co-localization with the Golgi. Since the Golgi is a major hub of intracellular trafficking, where most cargos travel through, it would make sense that many RABs are required to ensure efficient sorting. The precise localization of some of the RABs (RAB-1, RAB-8, RAB-10, RAB-18, RABY1, RAB-Y2 and GLO-1) could not be identified, as they showed only partial or no overlap with the markers tested. A tempting idea would be that some of these label novel compartments.

When compared with their mammalian orthologues, C. elegans RABs display conserved localization patterns. In agreement with our study, a recent analysis of the localization of Rabs in primary hippocampal neurons showed that many Rabs are localized to the perinuclear region most likely at the Golgi or endosomes (Pavlos et al., 2010). Additionally, Rab3 and Rab27 have been found to localize to synapses, Rab5 has been reported to localize to endosomes, Rab6 and Rab14 were shown to localize to the TGN-endosomal system (Griffiths et al., 1994; Pavlos et al., 2010; Stenmark et al., 1994; Mallard et al., 2002; Junutula et al., 2004).

Second to the nervous system, the $C$. elegans intestine expressed the largest number of Rabs. We are currently analyzing the localization of these intestinally expressed Rabs with the help of Barth Grant at Rutgers University, New Jersey, USA.

\subsection{RAB function in the $C$. elegans nervous system}

\subsubsection{Role of RABs in regulating simple behaviors}

After having established that RABs are predominantly found in neurons, we attempted to analyze their functions in these cells. This was carried out first by assaying several nervous system controlled behaviors: movement, defecation and egg- 
laying. Movement is controlled by a set of cholinergic and GABAergic motor neurons that function in parallel together (Fig. IV.6). Analysis of $r a b$ mutants showed that only rab-2 mutants were defective in movement. It has recently been shown that the movement defect of rab-2 mutant animals arises from a defect in dense core vesicle signaling (Sumakovic et al., 2009; Edwards et al., 2009).

Analysis of defecation revealed that a number of $r a b$ mutants had a severe decrease in expulsion efficiency. Among these were $r a b-2, r a b-7, r a b-10, r a b-19$, $r a b-27, r a b-28$ and $r a b-35$ mutants. $r a b-27$ also called aex-6 (aboc expulsion defective) has been previously identified in a screen for Aex mutants, but the precise role of RABs in regulating expulsion is unclear (Thomas, 1990). Expulsion is controlled by two motor neurons DVB and AVL (Fig. IV.8). It has been suggested that RAB-27 functions in the intestine to regulate the secretion of neuropeptides, which consequently activate the excitatory GABAergic DVB and AVL motor neurons (Mahoney et al., 2008). It is possible that additional RABs could participate together with RAB-27 to fine-tune this process. Since $r a b-27$ is also expressed in the nervous system and since it has been shown to be involved in synaptic vesicle exocytosis, it is possible that RAB-27 regulates the secretion of GABA directly from the DVB and AVL neurons. In such a scenario, the expulsion defective RABs, which were identified in this study, may also work alongside RAB-27 in SV exocytosis.

The length of the defecation cycle was also measured for each rab mutant and several mutants displayed increased cycle length while others had shorter cycle lengths. It has been demonstrated that cycle length is controlled by a calcium dependent mechanism. In synchrony with the pBoc, albeit occurring slightly earlier in time are a series of calcium transients in the intestine (Branicky and Hekimi, 2006). Mutations in an ER localized Inositol(1,4,5)trisphosphate $\left(\mathrm{IP}_{3}\right)$ receptor (ITR-1), displayed a loss of intestinal calcium transients and a consequent increased cycle length. Overexpression of ITR-1 led to shorter cycles (Dal Santo et al., 1999). Additional genes involved in the generation of $\mathrm{IP}_{3}$ such as phospholipase $\mathrm{C}$ (PLC) have also been implicated in regulating cycle length (Espelt et al., 2005). Rab GTPases have been shown to directly interact with phosphoinositide kinases and phosphatases (Shin et al., 2005; Stenmark, 2009). As a result of these interactions, they modulate the phosphoinositol pools in the cell. It is possible that rab mutants may change the levels of Phosphoinositol $(4,5)$ bisphosphate $\left(\mathrm{PIP}_{2}\right)$. Since $\mathrm{PIP}_{2}$ is a precursor for the formation of $\mathrm{IP}_{3}$, changes in $\mathrm{PIP}_{2}$ levels may affect the length of the 
defecation cycle. Interestingly $r a b-3, r a b-6, r a b-8$, and $r a b-35$ mutants displayed an increase in cycle length. The mammalian orthologues of these Rabs were shown to bind the inositol-polyphosphate 5-phosphatase, OCRL (Oculocerebrorenal syndrome of Lowe) (Fukuda et al., 2008).

Movement and defecation are primarily controlled by cholinergic and GABAergic neurons. To verify if RABs were also important in regulating monoamine transmitter release, we tested the $r a b$ mutants for defects in egg-laying. Egg-laying is mediated by the hermaphrodite specific neurons (HSNs) as well as VC neurons, both of which secrete serotonin (Schafer, 2005). The egg-laying assay revealed that $r a b$ 10 mutants displayed the strongest egg-laying defect. It is possible that RAB-10 may either regulate the development of the HSN and VC cells or be directly involved in the secretion of serotonin containing vesicles.

\subsubsection{Role of RABs in regulating synaptic transmission}

RAB-3 and RAB-27 have been shown to function together in regulating SV exocytosis at the C. elegans NMJ (Mahoney et al., 2006a). To investigate if additional RABs were involved in SV exocytosis, we used a pharmacological aldicarb based assay that allows an assessment of secreted ACh levels. Generally, aldicarb hypersensitivity suggests increased secretion, whereas aldicarb resistance suggests decreased secretion. However, sensitivities to aldicarb may also change as a consequence of an altered postsynapse. To distinguish between the two possibilities, aldicarb response assays are done in parallel to levamisole response assays. $r a b-3$ and rab-27 mutants were, as previously shown, less sensitive to aldicarb, but responded normally to levamisole. This suggests a decrease in SV release and correspondingly less ACh release. Previous aldicarb assays on $r a b-3$ and $r a b-27$ mutants support this (Mahoney et al., 2006b). In addition to these two rabs, rab-14 mutants were also aldicarb resistant. Interestingly rab-14 mutants also showed a resistance to levamisole suggesting fewer nAChRs at the postsynaptic muscle cell surface. It is possible that RAB-14 functions in the transport of nAChRs or perhaps in the degradation/recycling of these receptors. In accordance with this, it was recently shown that the mammalian orthologue of RAB-14 is indirectly involved in fibroblast growth factor receptor (FGFR) trafficking to the plasma membrane (Ueno et al., 2011).

Other $r a b$ mutants displayed hypersensitivity to aldicarb: $r a b-2, r a b-6.2, r a b$ 8, $r a b-35$ and $r a b-y 1$. Interesting, $r a b-2$ mutants were also hypersensitive to 
levamisole suggesting that in these mutants there is in increase in nAChRs at the postsynaptic side. This could occur due to a failure in efficient receptor degradation or recycling. It has been shown that at glutamatergic synapses, AMPA receptors are endocytosed and sorted into late endosomes for degradation or into recycling endosomes for replacement at the cell surface (Ehlers, 2000). It is possible that a similar mechanism exists at cholinergic synapses for nAChRs. RAB-2 has already been suggested to be involved in the recycling of AMPA receptors at glutamatergic synapses in C. elegans (Chun et al., 2008). The hypersensitive response of rab-6.2 mutants to aldicarb and levamisole is not likely to be due to changes in synaptic transmission. Observation of these mutants showed that they are very sick and burst upon reaching adulthood. Further experiments demonstrated that these mutants have blisters on their cuticula (data not shown). It is possible that a weaker cuticle would allow drugs to penetrate more easily, causing the animals to be hypersensitive.

The phenotype of rab-10 mutants was especially interesting because they displayed no change in aldicarb sensitivity, but were hypersensitive to levamisole. The hypersensitivity to levamisole is indicative of a postsynaptic defect. One possible explanation could be that the channel properties of the nAChRs are affected in $r a b-10$ mutants rendering them more sensitive to levamisole. This could potentially occur due to erroneous post-translational modifications as the receptors are transported to the cell surface or due to perturbed receptor recycling. In agreement with this, a previous study has implicated RAB-10 in AMPA receptor recycling in C. elegans neurons (Glodowski et al., 2007). It could also be that $r a b-10$ mutants have both, a presynaptic defect and a postsynaptic defect. In such a case, $r a b-10$ mutants could be secreting less ACh, but the results of the aldicarb assay would be masked by a hypersensitive postsynapse. Repeating these experiments with $r a b-10$ mutants rescued with rab-10 expression only in the muscles would help to differentiate these possibilities. $r a b-8, r a b-35$ and $r a b-y 1$ mutants showed hypersensitivity to aldicarb and normal sensitivity to levamisole. These results are indicative of increased levels of steady state SV exocytosis. It could be that the basal secretion levels are increased in these mutants.

\subsubsection{Role of RABs in regulating chemosensation}

Since a large portion of the C. elegans nervous system is dedicated to sensing the environment, we verified if $r a b$ mutants had obvious defects in the chemosensory 
system. DiI staining of the amphids and phasmids showed that all mutants were similar to wild type except $r a b-2$ mutants. $r a b-2$ mutants displayed normal dye filling in the amphids, but failed to efficiently fill in the phasmids. Interestingly, mutants with similar defects have been identified in dye filling screens (Herman and Horvitz, 1994). Interestingly, one such mutant, eor-1, has also been shown to have defects in movement, similar to rab-2 mutants. EOR-1 is a transcription factor important for cell differentiation (Hoeppner et al., 2004). Rab GTPases involved in membrane trafficking have previously been shown to bind effectors, which translocate to the nucleus to alter gene expression (Miaczynska et al., 2004). Additional experiments are necessary to understand if there is a link with RAB-2 and the regulation of gene expression.

It would also be interesting to test the functionality of the phasmid neurons in rab-2 mutants. It has been shown that these neurons, PHA and PHB, are negative modulators of sensory avoidance behaviors (Hilliard et al., 2002). Since we only tested for copper avoidance, which is mediated by ASH neurons, it would explain why no phenotype was observed in this assay. Interestingly, rab-10 mutants displayed a mild defect in copper avoidance. This may be due to a missorting of the GPCR necessary for copper sensation in these mutants (Bargmann, 2006).

\subsection{C. elegans RABs function redundantly together}

Knockout and knockdown analysis of mammalian Rabs showed a high degree of functional redundancy. Rab3A, Rab3B, Rab3C and Rab3D work together in SV exocytosis (Schlüter et al., 2004). Furthermore, Rab27A and Rab27B were shown to work together in dense granule secretion from platelets (Tolmachova et al., 2007). Based on these data, we thought initially that the C. elegans RABs may be less redundant due to its smaller family size. However, from the results of this study, it was striking to see that despite the smaller C. elegans RAB family, many of the $r a b$ mutants were healthy and few displayed strong defects. To understand which RABs were working together, a synthetic RNAi screen was conducted with the rab mutants. Each $r a b$ mutant was co-depleted with the remaining 27 rabs. Overall, the screen revealed that simultaneous removal of more than one RAB leads to more obvious phenotypic alterations. The strongest genetic interactions observed were synthetic lethalities. It was found that $r a b-8$ and $r a b-10$ mutants are synthetically lethal in a reciprocal manner. Additionally it was observed that $r a b-3$ and $r a b-19$ mutants were 
synthetically lethal with $r a b-21 \quad R N A i$. However, these interactions were not reciprocal. One possible explanation for this is that the RNAi of rab-3 and rab-19 was not efficient enough to cause a synthetic lethality. Therefore, in this case it would be a good idea to try other RNAi methods; RNAi by injection or soaking have been shown for certain genes to cause a better knockdown (Kamath et al., 2001; Tabara et al., 1998).

Other nervous system mediated phenotypes were also observed such as Unc and Egl. Unc was observed when $r a b-35$ mutants were knocked down with $r a b-7$. A double mutant of these two rabs recapitulated the RNAi phenotype (data not shown). Interestingly, $r a b-27$ mutants were Unc when co-depleted with $r a b-3$, in accordance with a previous analysis of these mutants (Mahoney et al., 2006a). Among all the rab mutants, $r a b-2$ animals displayed the largest number of genetic interactions with other rabs. Considering that RAB-2 is also the most highly conserved RAB GTPase across all species, it is likely that it is a central regulator of Golgi trafficking.

Out of interest, we also screened the $r a b-3 ; r a b-27$ double mutant to look for additional genetic interactions. We found that $r a b-3$; $r a b-27$ double mutants, which were co-depleted with $r a b-37$, were more severely Unc. This suggests that RAB-37 may have a previously unidentified role in regulating SV release. Interestingly, rab37 displayed very specific expression in neurons similar to $r a b-3$ and $r a b-27$. Additionally all three of these genes are classified in one functional group by virtue of structural similarities (Pereira-Leal and Seabra., 2001).

Through these screens we have identified novel combinations of RABs that may function together. In a next step it is necessary to validate and characterize these interactions using the mutants available to gain a more mechanistic understanding of how different RABs work together. It is also conceivable that double mutants can additionally be screened to identify triply redundant RABs. 


\section{CHAPTER 2:}

\section{REGULATION OF DENSE CORE VESICLE SECRETION BY RAB GTPASES IN C. ELEGANS}




\section{INTRODUCTION}

As the second chapter of the thesis will deal with the role of RAB GTPases in regulating dense core vesicle (DCV) secretion C. elegans, a brief introduction into the protein machinery behind SV and DCV exocytosis is provided.

\subsection{The SV exocytosis machinery}

Neurotransmitter release through SV exocytosis is the principle mode of intercellular communication in the brain. During this process, SVs filled with neurotransmitters move towards the presynaptic active zone, where they dock with the plasma membrane. After docking, SVs prepare for release through a process called priming, which renders them more competent to fuse. Upon an influx of calcium, vesicles fuse with the plasma membrane. After a fusion event, SVs are recycled most likely by endocytosis (Fig. VI.1) (Richmond, 2005). A number of critical proteins, in addition to Rabs, have been identified to make up the SV release machinery: Synaptobrevin-2, syntaxin-1, SNAP-25, Munc18, synaptotagmin-1, Munc13, complexin, tomosyn, and CAPS $\left(\mathrm{Ca}^{2+}\right.$-dependent activator protein for secretion) (Richmond, 2005; Soerensen, 2009). These components are shown in figure VI.1.

SNAREs were the first basic components identified shown to be necessary for fusion (Weber et al., 1998). Synaptobrevin-2 is an R-SNARE localized on the membrane of synaptic vesicles. Syntaxin-1 (Qa SNARE) and SNAP-25b (Qbc SNARE) are localized at the plasma membrane of presynaptic terminals (Sutton et al., 1998; Fasshauer et al., 1998). These three SNAREs are capable of assembling rapidly into a trans-SNARE complex via an $\mathrm{N}$ to $\mathrm{C}$ terminal zippering mechanism (Pobbati et al., 2006). The assembly of $R$ and Q SNAREs into a four helical bundle 
A

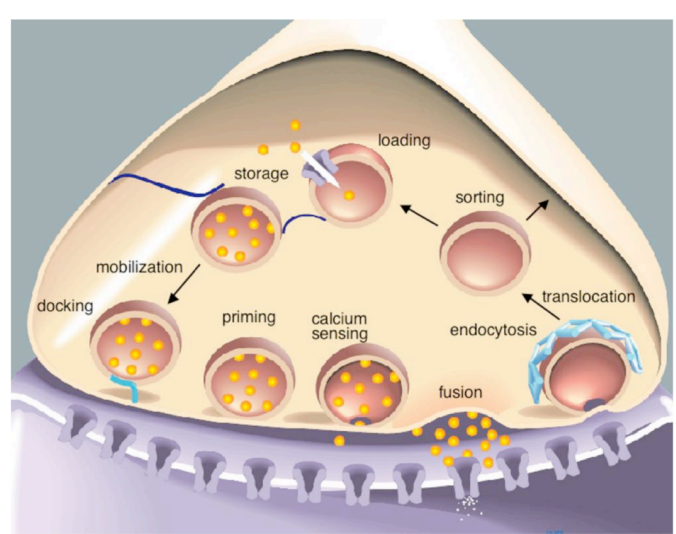

$\mathrm{B}$

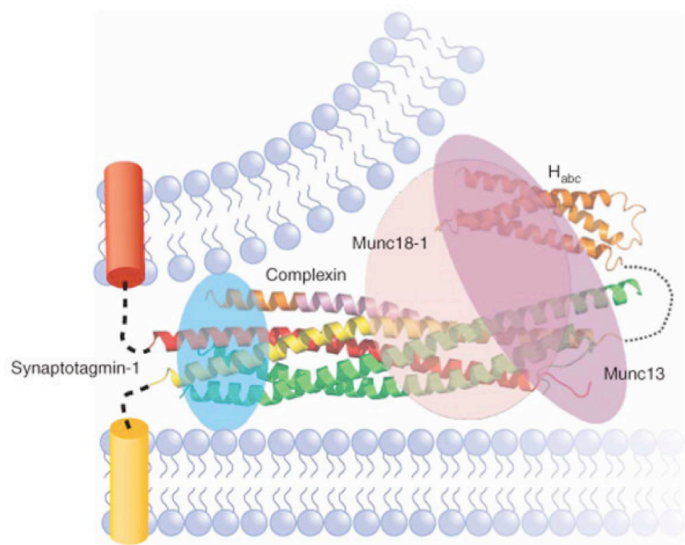

Figure VI.1. The synaptic vesicle cycle and its machinery. A. Synaptic vesicles are loaded with neurotransmitter at the synapses and subsequently undergo fusion at the presynaptic terminal. Fusion has been described to occur through docking, priming, calcium sensing and subsequent fusion. The discreteness of these steps is becoming more clear as better understandings of the protein machinery are emerging (Richmond, 2005) . B. A number of proteins are implicated in SV docking, priming and fusion: SNAREs, Munc18-1, Synaptotagmin-1, Munc-13-1 and complexin. CAPS and tomosyn have also shown to be involved, but are not depicted above (Rizo and Rosenmund, 2008). Source: Pictures taken from Richmond, 2005; Rizo and Rosenmund, 2008.

is thought to provide the energy necessary for membrane fusion (Jahn and Scheller, 2006).

Munc18 is a $60 \mathrm{kDa}$ cyotosolic protein, which has been shown to bind to the closed conformation of Syntaxin-1 (Habc domain closed onto the SNARE domain) (Dulubova et al., 1999). However, more recently it has been shown that Munc18 can also bind to the assembled SNARE complex through a previously unidentified Nterminal peptide of Syntaxin-1a (Shen et al., 2007; Burkhardt et al., 2008). The mechanism of action on SNARE assembly is a subject of controversy as results from in vitro studies yield conflicting data. Shen et al. suggested that N-peptide binding accelerates SNARE complex assembly, whereas Burkhardt et al. suggest that abolishing the N-peptide interaction allows SNARE complex formation (Shen et al., 2007; Burkhardt et al., 2008). In vivo studies have showed that deletion of Munc18 in mice leads to a sharp decline in SV release suggesting that Munc18 has a stimulatory function for SNARE driven exocytosis (Verhage et al., 2000). The precise sequence of events in which Munc18 interacts with Syntaxin-1 and the SNARE complex remains to be elucidated.

Early studies by Katz showed that the release of SVs is triggered by the influx of $\mathrm{Ca}^{2+}$ (Katz, 1969). This process intuitively demanded the requirement of a calcium sensor. Since then, it has been determined that $\mathrm{C} 2$ domain containing proteins called 
synaptotagmins $(65 \mathrm{kDa})$ could take up this role. $\mathrm{C} 2$ domains possess dual functions: $\mathrm{Ca}^{2+}$ binding $(\sim \mathrm{Kd} 50 \mu \mathrm{M})$ as well as phospholipid binding (Nalefski et al., 2001). Considering also that synaptotagmin-1 (syt-1) localized to SVs, syt-1 was proposed to be the major calcium sensor. In accordance with this, mice knockouts of syt-1 revealed that it is essential for synchronous evoked SV release (Geppert et al., 1994; Chapman, 2008). Interestingly there are 16 mammalian synaptotagmins, the majority of which remain functionally uncharacterized (Pang and Südhof, 2010).

Munc13s are large $(\sim 200 \mathrm{kDa})$ multi domain containing proteins. Munc13-1 is involved in SV priming (Augustin et al., 1999). It contains a $\mathrm{C} 1$ domain, three $\mathrm{C} 2$ domains (C2A, C2B and $\mathrm{C} 2 \mathrm{C})$, a calmodulin ( $\mathrm{CaM})$ binding domain and a MUN domain (Fig. VI.2) (Brose et al., 1995; Shin et al., 2010). The C1 domain was shown to act as a receptor for binding phorbol esters such as diacylglycerol (DAG) (Ahmed et al., 1992; Betz et al., 1998). The three C2 domains are thought to differ in their functions (Brose et al., 1995). Recently it was shown that only the C2B is important for binding calcium $(<\mathrm{Kd} 100 \mu \mathrm{M})$ and phosphoinositolphosphate lipids (Shin et al., 2010). The CaM domain was also shown recently to bind to $\mathrm{Ca}^{2+}-\mathrm{CaM}$, which is thought to further facilitate SV priming (Rodriguez-Castaneda et al., 2010). Finally, the MUN domain is thought to directly or indirectly bind and open syntaxin-1 (Habc domain from SNARE domain) to allow for SNARE complex assembly (Madison et al., 2005; Richmond et al., 2001; Basu et al., 2005). However, the biochemical mechanism of how the MUN domain of Munc13 regulates SNARE assembly and also how it works in concert with Munc18 remains unclear. Interestingly the N-terminus of Munc-13 has also been shown to bind the Rab3 effector, RIM1 (Betz et al., 2001; Wang et al., 1997). In vivo deletions of Munc-13 or RIM1 lead to similar decreases in SV release (Varoqueaux et al., 2002; Schoch et al., 2006). Furthermore,

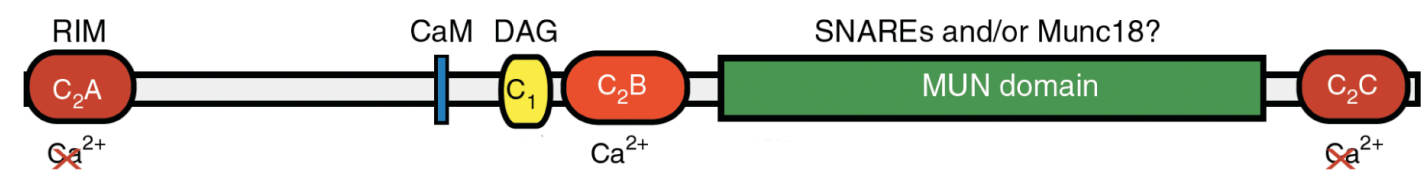

Figure VI.2. Domain structure of Munc13-1. Munc13-1 is a multidomain protein. It consists of a $\mathrm{C} 1$ domain important for $\mathrm{DAG}$ binding, three $\mathrm{C} 2$ domains of which $\mathrm{C} 2 \mathrm{~B}$ is important for calcium binding, a CaM domain important for binding calmodulin and a MUN domain which is suggested to regulate SNARE assembly. Source: Picture taken and adapted from Shin et al., 2010. 
RIM1, like Munc13, is also thought to be necessary for opening syntaxin-1 (Koushika et al., 2001).

Complexins are small $15 \mathrm{kDa}$ cyotosolic proteins (complexin 1 and 2) that were identified to bind with high affinity to the assembled SNARE complex in a 1:1 ratio (McMahon et al., 1995; Chen et al., 2002). The role of complexins on SV release has been studied in a wide variety of model systems, yielding conflicting data. However, in general it has been described that changes in cellular levels of complexin (overexpression or depletion) leads to a decrease in spontaneous and evoked exocytosis (Brose, 2008). Furthermore, from mouse knockout studies it is clear that the function of complexin is carried out through its binding to the assembled SNARE complex (Reim et al., 2001; Xue et al., 2007). Studies with complexin mutants in $D$. melanogaster and C. elegans have revealed similar results for complexins' role in evoked release, but a contradictory increase in spontaneous release (Huntwork and Littleton, 2007; Hobson et al., 2011). Interestingly knockdown, as opposed to knockout, of complexin in mice reveals similar phenotypes as in flies and worms (Yang et al., 2010). This suggests that complexin may have an additional clamp function to minimize calcium-independent secretion events. With respect to the other components of SV fusion, it is thought that complexin binds to the SNARE complex keeping it in a primed state and that calcium entry triggers complexin displacement by synaptotagmin (Brose, 2008).

Tomosyn is a $130 \mathrm{kDa}$ cyotosolic protein that is found to be an inhibitor of $\mathrm{SV}$ release. It consists of two major domains: an N-terminal domain with WD40 repeats as well as a C-terminal R-SNARE domain. The inhibitory action of tomosyn was identified to be a result of competition with the neuronal R-SNARE, synaptobrevin-2, for SNARE assembly leading to less fusion (Hatsuzawa, 2003). The TomosynSNARE complex and the Synaptobrevin-SNARE complex represent end products of the assembly reaction. The N-ethylmaleimide-sensitive factor (NSF) is necessary for disassembly (Pobbati et al., 2004). In accordance with this role of tomosyn, in vivo studies have also shown that deletion of tomosyn leads to more sustained SV release (Hatsuzawa, 2003; Gracheva et al., 2007).

CAPS is a $145 \mathrm{kDa}$ cyotosolic protein originally identified to be a component of the DCV secretion machinery (Walent et al., 1992). Since then it has also been shown to be control SV release (Jockusch et al., 2007). CAPS is a multidomain 


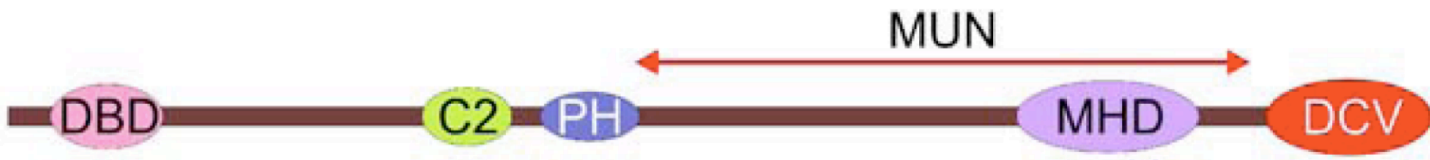

Figure VI.3. Domain structure of CAPS-1. CAPS is also a multidomain protein. At the N-terminus it has a dynactin binding domain (DBD). This is followed by a C2 domain, a PH domain a MUN domain and a C-terminal DCV binding domain. Source: picture taken from Stevens and Rettig, 2009.

protein containing an $\mathrm{N}$-terminal dynactin binding domain (DBD), followed by a $\mathrm{C} 2$ domain, a Pleckstrin homology $(\mathrm{PH})$ domain, a MUN domain and a C-terminal DCV binding domain (Fig. VI.3). Not much is known about the DBD except that it is likely to have a sorting function. The $\mathrm{C} 2$ domain is suggested to be important for $\mathrm{Ca}^{2+}$ mediated membrane binding, although it was showed to have only moderate affinity for $\mathrm{Ca}^{2+}(\sim \mathrm{Kd} 270 \mu \mathrm{M})$ (Ann et al., 1997). The PH domain was identified to be important for membrane binding, perhaps through cooperating with the $\mathrm{C} 2$ domain (Grishanin et al., 2002). The MUN domain of CAPS is similar to the MUN domain of Munc13 and is thought to be perhaps important for SV priming via interaction with SNAREs (Stevens and Rettig, 2009). The C-terminal DCV domain has been shown to be necessary for localization onto DCVs (Grishanin et al., 2002). In vivo analysis of CAPS knockout mice (double CAPS1 and CAPS2 knockout), have revealed that CAPS1 is essential for evoked SV release and is partially redundant with CAPS2 (Jockush et al., 2007). It has been suggested that it may also participate in opening syntaxin-1, together with Munc13, although electrophysiological evidence for this is not yet available for neurons (Hammarlund et al., 2008).

The exact sequence in which all of the SV exocytosis machinery work together remains still a mystery. Currrent working models with some of these factors suggest that as SVs approach the active zone, docking is likely mediated by a Rab GTPase (Rab3 / Rab27) in an interaction with a Rab effector (RIM-Munc13) in complex with SNAP-25, syntaxin-1, Munc18 (the acceptor complex). After docking synaptobrevin on SVs binds to the acceptor complex with its N-terminus to form an intermediate SNARE complex, where the C-terminus of synaptobrevin would be kept unstructured. At this point, it is also thought that synaptotagmin and complexin bind to this intermediate SNARE complex stabilizing it to facilitate priming. Upon an influx of calcium, complexin is displaced and synaptotagmin is thought to provide the force for the final fusion and full SNARE assembly (Sorensen, 2009; Brose, 2008). 


\subsection{The DCV exocytosis machinery}

In contrast to SVs, dense core vesicles are loaded with bioactive peptides and function to modulate fast synaptic transmission. Most of our current understanding of the DCV secretion machinery comes from experiments conducted on neuroendocrine chromaffin cells of the peripheral nervous system. These cells contain mostly large DCVs $(\sim 200 \mathrm{~nm})$ that fuse in a calcium dependent manner. These events are detectable through capacitance measurements and amperometry (Neher and Marty, 1982; Chow et al., 1994; Voets, 2000). Genetic analysis in chromaffin cells has revealed that many of the components necessary for SV release are also necessary for DCV exocytosis. First it has been shown that the same SV SNAREs were necessary (Xu et al., 1998). Subsequently, the need for Munc18-1, synaptotagmins, Munc13-1, complexin, tomosyn and CAPS have also been elucidated for DCV secretion (Voets et al., 2001; Schonn et al., 2008; Ashery et al., 2000; Cai et al., 2008; Yizhar et al., 2004; Liu et al., 2010). Interestingly, a new specific role of CAPS function at the TGN has been suggested (Sadakata et al., 2010). It was shown that CAPS also localizes to the cell body and regulates DCV release through an interaction with many Golgi localized proteins such as Arf GTPases (Sadakata et al., 2010).

\subsection{DCV biogenesis and maturation}

Despite similarities in their exocytic machinery, SV and DCV turnover occurs through entirely different mechanisms. After release SVs are recycled through local endocytosis (Südhof, 2004). DCVs, on the other hand, must be synthesized de novo at the TGN (Kim et al., 2006; Morvan and Tooze, 2008). At the TGN, DCVs are initially generated in an immature form (iDCVs). Prior to reaching the synapse, these iDCVs undergo a maturation process whereby neuropeptides are retained and lysosomal enzymes, M6P receptors and constitutive secretory cargo is removed (Fig. VI.4). The removal of components during maturation is thought to occur through clathrin coated vesicle (CCV) sorting (Tooze et al., 2001; Lui-Roberts et al., 2005). Before maturation into $\mathrm{mDCVs}$, iDCVs also undergo a process of homotypic fusion where vesicle size is increased or decreased (Tooze et al., 1991). Interestingly several proteins have been identified specifically to localize to iDCVs and not on mDCVs: Syntaxin 6, VAMP 4 and Synaptotagmin IV (Wendler et al., 2001; Eaton et al., 2000). Additionally, during the process of maturation, iDCVs are acidified and 


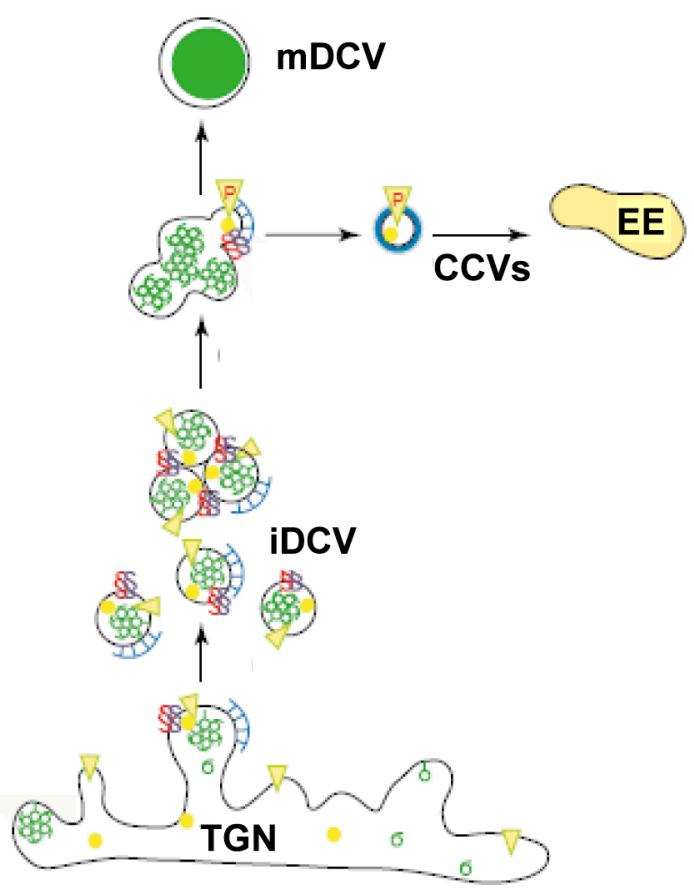

Figure VI.4. DCV maturation. Before reaching the synapse DCVs must undergo a maturation process. Maturation of iDCVs to $\mathrm{mDCVs}$ requires the retention of neuropeptides, and respective processing enzymes and parallel removal of cargo such as lysosomal hydrolases and M6P receptors. Removal of components is suggested to occur via a CCV pathway to the endosomes. During maturation the size of iDCVs becomes larger or smaller as a consequence of homotypic fusion events (Tooze et al., 2001). Source: Picture taken and adapted from Tooze et al., 2001.

propeptides are cleaved at mono- or di-basic residues $(-\mathrm{K} / \mathrm{-R})$ through the action of proprotein convertases to form active peptides (Rouille et al., 1995). This endoproteolysis produces short peptides with C-terminal basic resides, which are subsequently cleaved by carboxypeptidase E (Rouille et al., 1995).

\subsection{DCVs function to regulate synaptic transmission}

Although, it is clear that DCVs are modulators of synaptic transmission, the precise function of their peptide load remains to a large extent unknown. There are approximately 100 neuropeptide encoding genes identified in the human genome (Burbach, 2010). Recent work has suggested that certain neuropeptides can alter spontaneous and evoked release of SVs through a G-protein coupled receptor (GPCR) pathway. In this model, neuropeptides would activate a Gaq-PLC pathway (in a manner similar to monoamine neurotransmitters). This would consequently lead to the increased production of DAG and $\mathrm{IP}_{3}$ from $\mathrm{PIP}_{2}$ (Perez-Mansilla and Nurrish, 2009; Lackner et al., 1999). Since Munc13 contains a phorbol ester-binding domain, increased DAG levels lead to an increased level of SV release (Rhee et al., 2002; Lou et al., 2008). This stimulation of SV release is counter-balanced by a Goo pathway 


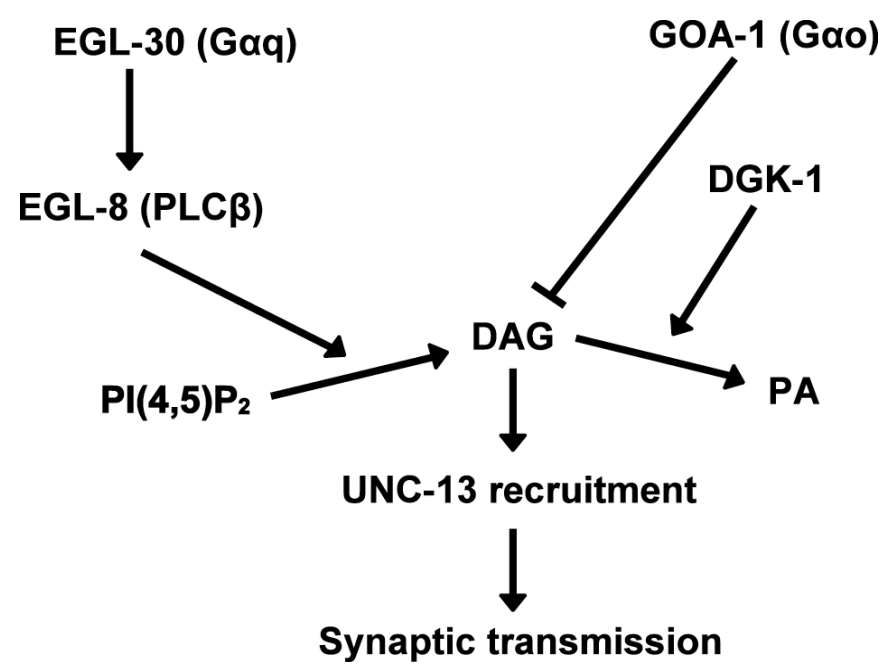

Figure VI.5. Regulation of SV release by GPCRs. Neuropeptides are thought to activate the GaqPLC pathway, which leads to an increased production of DAG. DAG consequently binds to the C1 domain of UNC-13 and potentiates SV release. This pathway is counterbalanced by a Goo pathway and DGK-1 (C.e orthologue of DAG kinase). DGK-1 phosphorylates DAG to generate phosphatidic acid (PA). Note that these pathways can also be activated by monoamine neurotransmitters (PerezMensilla and Nurrish, 2009). Source: Picture taken and adapted from Perez-Mansilla and Nurrish, 2009 .

and DAG kinase (DGK) which downregulates DAG levels. Figure VI.5 illustrates this pathway depicting the C. elegans orthologues of the molecules involved (many of the components were identified initially through screens in C. elegans) (Perez-Mansilla and Nurish, 2009). To date, the molecular identity of the neuropeptide GPCR of this pathway remains elusive and is a topic of much attention.

\subsection{Studying DCV function in C. elegans}

C. elegans has proven to be a useful system for the genetic dissection of DCV function. Like in the mammalian system, approximately 100 neuropeptide precursor genes have been identified in the nematode genome. Furthermore, much of the protein machinery involved in DCV maturation and release is conserved ( $\mathrm{Li}$ and $\mathrm{Kim}, 2008$ ). Recently a fluorescent-based assay to study DCV function in vivo has been developed in C. elegans (Fig.VI.6) (Sieburth et al., 2007). A neuropeptide (NLP-21) tagged to yellow fluorescent protein (YFP) is expressed in C. elegans DA and DB cholinergic motor neurons. The NLP-21-YFP is packaged into DCVs and transported to the NMJ. Prior to reaching the synapse, like other neuropeptides, NLP-21 is cleaved from the proform into the short bioactive form through the action of proprotein convertases 
A

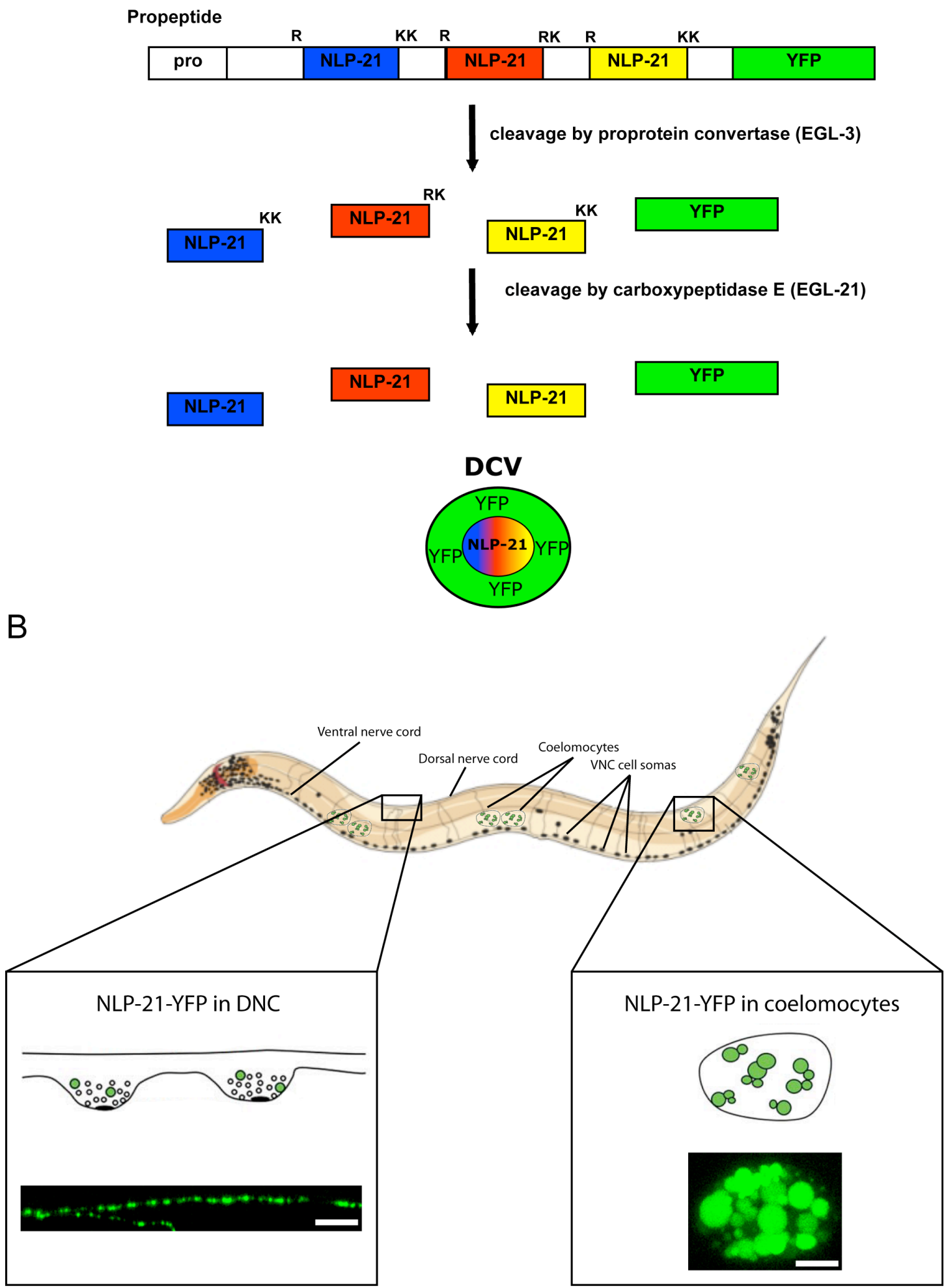

Figure VI.6. Studying DCV function in C. elegans. A. DCV trafficking can be studied using a fluorescence-based assay in C. elegans. A construct containing neuropeptide tagged to YFP (NLP-21YFP) is used. NLP-21 is processed like other peptides and packaged into DCVs. B. NLP-21 is expressed in the cholinergic motor neurons of the worm (using the unc-129 promoter). NLP-21-YFP is trafficked to the DNC NMJs and released into the body cavity. Scavenger cells called coelomocytes endocytose the YFP. Fluorescence intensity in the coelomocytes is used as an indirect readout of secretion. Fluorescence intensity in the DNC is also analyzed to measure the transport of DCVs to the NMJ. Scale bar in DNC represents $6 \mu \mathrm{m}$ and bar in coelomocytes is $3 \mu \mathrm{m}$ (Sieburth et al., 2007). Source: Pictures taken and adapted from Gracheva et al., 2007; Sumakovic et al., 2009. 
(EGL-3) and carboxypeptidase E (EGL-21). After DCV maturation the NLP-21-YFP peptides are released into the synaptic extracellular milieu (ECM). C. elegans possesses 6 macrophage-like cells called coelomocytes which continuously filtrate the body fluid of the pseuodocoelem through bulk endocytosis. The amount of YFP endocytosed by these coelomocytes can be measured as an indirect reporter of DCV secretion levels. Additionally YFP levels can, in parallel, be measured in the synapses of the motor neurons to analyze DCV trafficking (Sieburth et al., 2007). Strikingly, most mutants, which displayed defects in DCV release in the mammalian system, have also been detected through this assay: CAPS (C.e UNC-31), Munc-13 (C.e UNC-13), tomosyn (C.e TOM-1) (Sieburth et al., 2007; Gracheva et al., 2007).

In a previous project from our laboratory, this assay was used to detect a DCV maturation defect in rab-2 mutants in C. elegans (Sumakovic et al., 2009). To our surprise, $r a b-2$ mutants displayed a genetic interaction with $r a b-5$ in DCV function. This led us to believe that multiple RAB GTPases may coordinate together to regulate DCV trafficking. 


\section{AIMS}

A large cohort of proteins are necessary for the regulation of DCV function. Some of these molecules, such as Munc18 and Munc13 were first identified in $C$. elegans (Maruyama and Brenner, 1991; Hosono et al., 1992; Brenner, 1974). The recent development of novel in vivo imaging assays has permitted more rigorous analyses of DCV function in C. elegans (Fig. VI.6) (Sieburth et al., 2007). Although, the NLP-21-YFP assay developed by Sieburth et al. is an indirect means to measure DCV function, many previously identified factors involved in DCV secretion were shown defective also in NLP-21-YFP secretion: CAPS (C.e UNC-31), Munc-13 (C.e UNC-13), tomosyn (C.e TOM-1) (Sieburth et al., 2007; Gracheva et al., 2007).

This assay recently allowed for the elucidation of RAB-2 as a regulator of DCV function (Sumakovic et al., 2009; Edwards et al., 2009). rab-2 mutants displayed decreased NLP-21-YFP levels in the DNC compared to wild type animals (approximately $20 \%$ of wild type). Since RAB-2 localizes to the Golgi, it was established that it plays an early role in the maturation of DCVs by ensuring that necessary cargo is retained (Sumakovic et al., 2009; Edwards et al., 2009). In rab-2 mutants, it was thought that there is a loss of DCV cargo into the endo-lysosomal system. Therefore, to block this loss of cargo, a constitutively active mutant of $r a b-5$ $(Q 78 L)$ was overexpressed in a rab-2 mutant background. Interestingly, the $r a b-5$ (Q78L) suppressed the decreased levels of NLP-21-YFP in the DNC of rab-2 mutant animals (Fig. VII.1). These results showed, for the first time, that RAB GTPases cooperate in regulating DCV trafficking in C. elegans.

Considering that there are $28 \mathrm{RABs}$ encoded in the $C$. elegans genome and that mutants for these RABs were available, we aimed to determine novel Rabs involved in the regulation of DCV secretion. Since we had mutants for several 


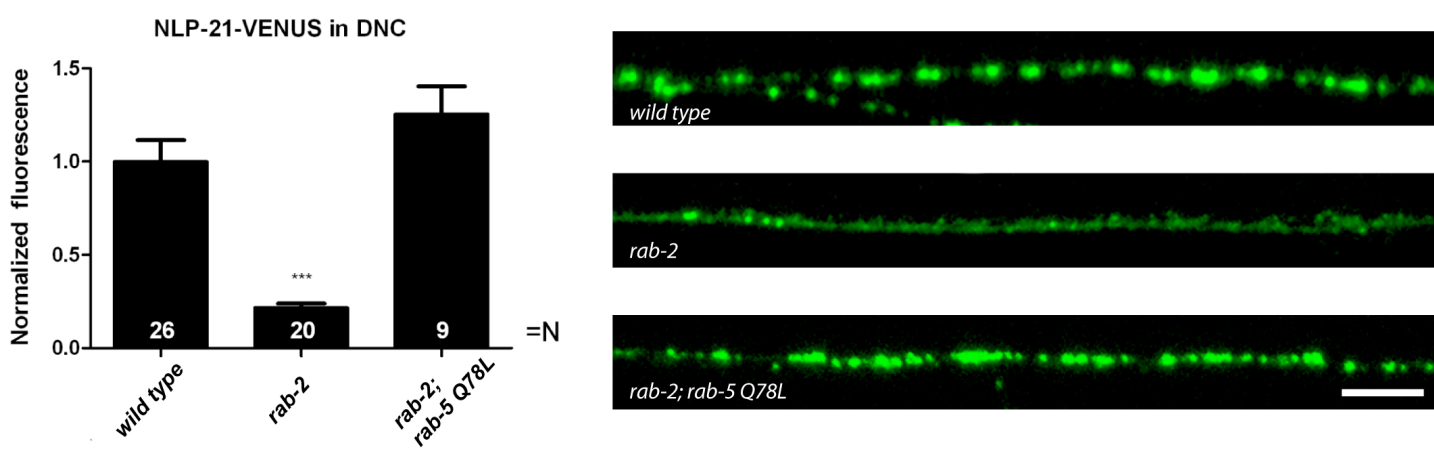

Figure VII.1. A rab-5 mutant suppresses DCV defects in $r a b-2$ mutants. $r a b-2$ mutants were found to display a defect in DCV maturation. The NLP-21-YFP levels in these strains were strongly decreased in the DNC. Strikingly when a constitutively active rab-5 (Q78L) mutant was expressed in a rab-2 mutant background, the NLP-21-YFP levels in the DNC returned to wild type levels. Scale bar in DNC represents $8 \mu \mathrm{m}$. Error bars $=\mathrm{SEM}(* * *, \mathrm{P}<0.005$ ANOVA with Bonferroni post test) (Sumakovic et al., 2009) Source: This experiment was not conducted in this study and is only used as a means to explain the rationale of the project. The original data for this figure was kindly provided by Marija Sumakovic.

regulators of RAB function (TBC domain containing RABGAPs), we also analyzed the role of these molecules in DCV secretion. After identification of new RABs and RABGAPs, we determined if they were DCV-specific regulators or if they were also involved in the regulation of SV secretion. As a last step we investigated how the function of all molecules may be integrated together to regulate secretion.

It must be mentioned that most experiments in this chapter were done together by the author and by Marija Sumakovic, as this was a shared project. The author was involved together with Marija Sumakovic in conducting experiments and producing the data for every figure, unless otherwise stated in the figure legend. 


\section{RESULTS}

\subsection{RAB-5 and RAB-10 are novel RABs essential for DCV release}

In order to identify if additional RABs were involved in DCV trafficking we tested all $r a b$ mutants through the NLP-21-YFP assay (Fig. VIII.1). The $r a b$ genes with no available mutant, were tested by RNAi knockdown and data from glo- 1 and rab-y1 to rab-y6 were excluded for simplicity. Please note that knockdown of lethal genes ( $r a b-1, r a b-7, r a b-11.1)$ were conducted using a tissue specific RNAi technique to bypass the lethality and analyze adult worms. This technique is described in section 8.2. A rab-5 (Q78L) was used since it was available from a previous study (Sumakovic et al., 2009). Analysis revealed that both, an overexpression of $r a b-5$ $(Q 78 L)$ and the rab-10 deletion mutants displayed severe defects in NLP-21-YFP release. In contrast to rab-2 mutants, the rab-5 (Q78L) and the rab-10 mutants had wild type levels of YFP in the DNC, but drastically decreased levels in the coelomocytes. Coelomocyte fluorescence levels were $16.76 \pm 0.04 \%$ and $0.00 \pm 0.00$ $\%$ in $r a b-5(Q 78 L)$ and $r a b-10$ mutants respectively when compared to wild type. unc-31 mutants were used as a control and displayed a significant increase in the DNC with $159.60 \pm 0.18 \%$ and a significant decrease in the coelomocytes to $30.36 \pm$ $0.08 \%$ of wild type. From this point on, the overexpressed rab-5 $(Q 78 L)$, will be referred to as rab-5 (Q78L) mutants. To ensure that the defects that we observed were cell autonomous, we generated transgenic lines of rab-10 mutants expressing mCherry-rab-10 in the cholinergic DA and DB motor neurons. Transgenic lines rescued the YFP intensity levels in the coelomocytes of rab-10 mutants, supporting the idea that RAB-10 is indeed a regulator of DCV secretion (data not shown). 
NLP-21-YFP

Dorsal Nerve Cord

$\square$ Coelomocytes
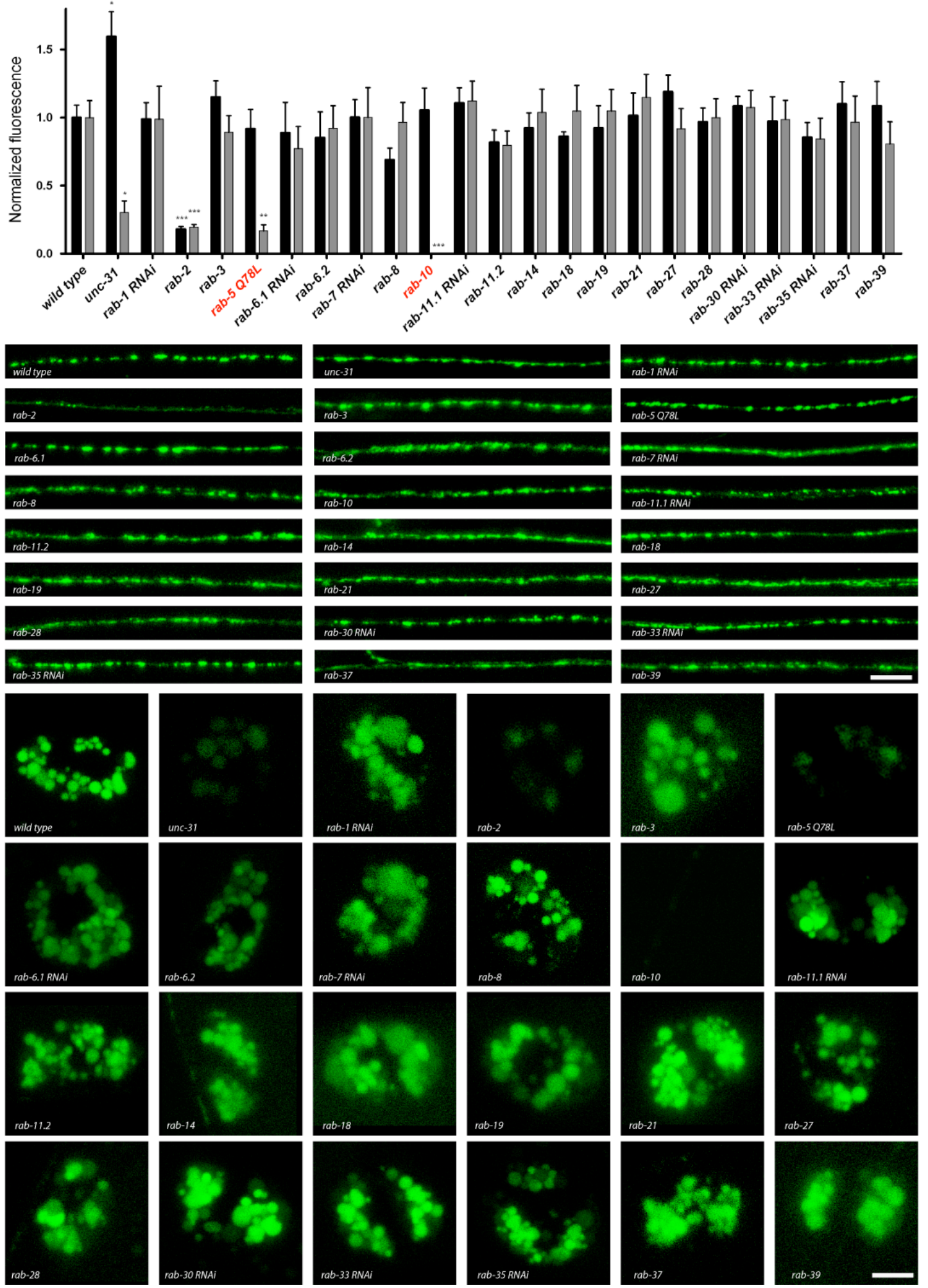

Figure VIII.1. NLP-21-YFP analysis of all rabs. The trafficking of NLP-21-YFP was analyzed for all $r a b$ mutants. The assay revealed that $r a b-5(Q 78 L)$ and $r a b-10$ mutants display a severe defect in DCV release. Scale bar in DNC represents $6 \mu \mathrm{m}$ and bar in coelomocytes is $3 \mu \mathrm{m}$. At least $\mathrm{N}=10$ animals were tested per strain. Error bars $=\operatorname{SEM}(* * *, \mathrm{P}<0.005 ; * *, \mathrm{P}<0.01, *, \mathrm{P}<0.05$ ANOVA with Bonferroni post test). Note: RNAi experiments were normalized to mock, L4440, knockdown. 


\subsection{DCV trafficking defects are autonomous to the nervous system}

\subsubsection{Development of a neuron-specific RNAi system}

NLP-21-YFP analysis revealed that $r a b-5$ (Q78L) and rab-10 mutants have defects in DCV release. Since rescue experiments were only feasible with rab-10 mutants and not rab-5 (Q78L) mutants, we developed a neuron-specific RNAi technique to determine cell-specificity of the observed DCV release defects.

A

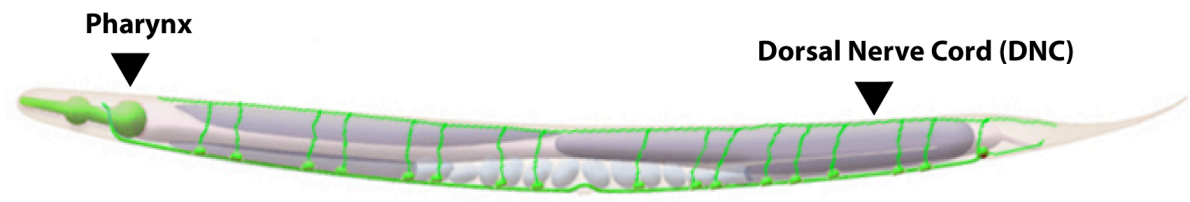

B

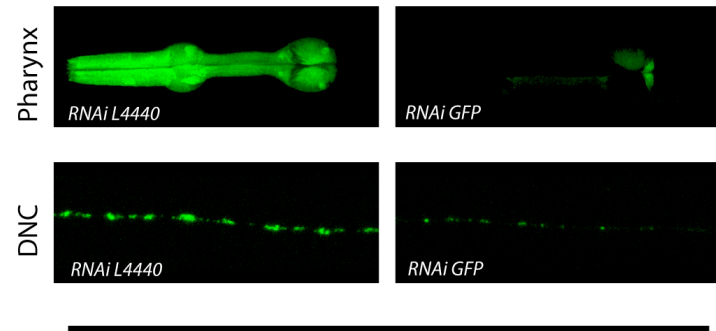

eri-1;nuls183
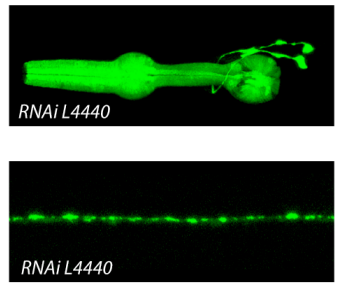
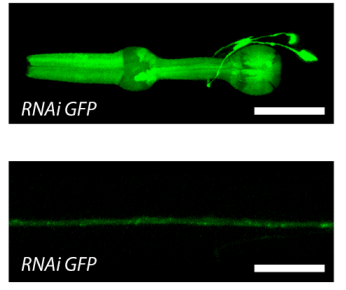

eri-1;rde-4;nuls 183

Ex [punc-129::rde-4]

Pharynx

Dorsal Nerve Cord
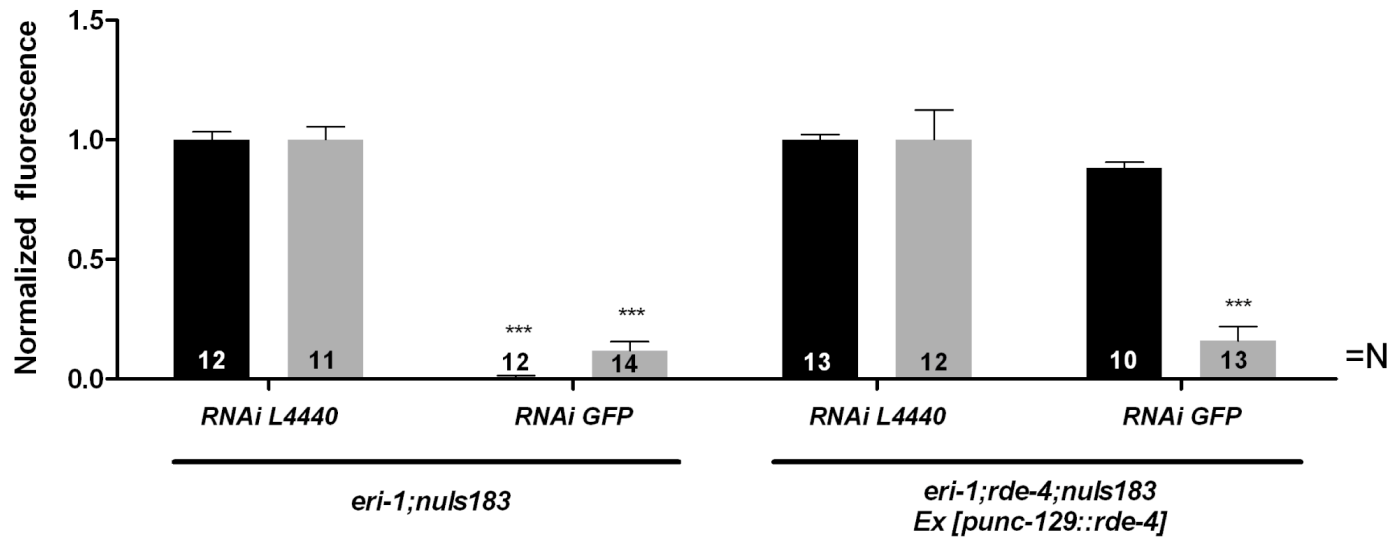

Figure VIII.2. Validation of neuron-specific RNAi. Neuron-specific knockdown was achieved using an $r d e-4$ mutant, which is deficient in RNAi. These mutants were rescued with $r d e-4$ expression only in the DA and DB motor neurons using the punc-129 promoter (Note: transgenic animals express pttx$3: \because g f p$ in head neurons. This was used as a co-injection marker). All RNAi experiments were done in the classical eri-1 background for enhanced sensitivity to RNAi and also in the nuIs 183 background, nuIs183 expresses GFP in the pharynx (selection marker) and NLP-21-YFP (assay marker). Knockdown of GFP revealed that RNAi was neuron-specific. Scale bar in pharynx represents $50 \mu \mathrm{m}$ and bar in DNC is $6 \mu \mathrm{m}$. Error bars $=\operatorname{SEM}(* * *, \mathrm{P}<0.005$; ANOVA with Bonferroni post test) 
RDE-4 encodes a double stranded RNA binding protein, which is important for stabilizing DNA for recognition by dicer. $r d e-4$ (RNAi deficient-4) mutant animals exhibit an inability to carry out RNAi (Duchaine et al., 2006). Therefore, using an $r d e-4$ mutant background, we transgenically rescued $r d e-4$ expression only in the DA and DB cholinergic motor neurons. This strain was then crossed into an eri-1 background (classical mutant background with increased RNAi sensitivity) and also the NLP-21-YFP assay strain (nuIs183) (Timmons, 2004; Sieburth et al., 2007). The nuIs 183 strain expresses GFP in the pharynx (myo-2 promoter) and the NLP-21-YFP in the DA and DB cholinergic motor neurons (unc-129 promoter). To validate if the tissue-specific RNAi was working, we conducted a control experiment knocking down GFP. Knockdown of GFP just in the eri-1; nuIs183 background led to significantly decreased XFP levels in the pharynx and the DNC. Fluorescence intensity was decreased to $0.83 \pm 0.58 \%$ and $11.76 \pm 3.75 \%$ in the pharynx and DNC respectively when compared to knockdown with a mock vector (L4440). Surprisingly, knockdown of GFP in the tissue specific RNAi strain, eri-1; rde-4; nuIs183 Ex[punc129::rde-4], lead to no significant decrease of XFP levels in the pharynx, but a reduction in the dorsal nerve cord. Fluorescence intensity values were $85.31 \pm 2.57 \%$ and $15.89 \pm 6.10 \%$ in the pharynx and DNC respectively when compared to L4440 knockdown (Fig.VIII.2).

\subsubsection{RAB-5 and RAB-10 cell-specifically regulate DCV release}

After having validated that the neuron-specific RNAi strain was working. We tested if we could phenocopy the rab-5 (Q78L) and rab-10 mutant phenotypes using RNAi. Interestingly, knockdown of $r a b-5$ and $r a b-10$ in the cholinergic motor neurons led to a similar significant decrease in fluorescence levels in the coelomocytes and normal levels in the DNC. Knockdown of $r a b-5$ and $r a b-10$ led to coelomocyte fluorescence intensities of $12.78 \pm 0.05 \%$ and $0.01 \pm 0.00 \%$ respectively compared to mock (Fig. VIII.3). This suggested that RAB-5 and RAB-10 play a cell autonomous function in regulating DCV secretion. Interestingly, further evidence which suggested that our tissue-specific RNAi was working was the ability of rab-5 RNAi animals to bypass lethality. Normal knockdown of rab-5 leads to late embryonic lethality. 


\section{NLP-21-YFP}
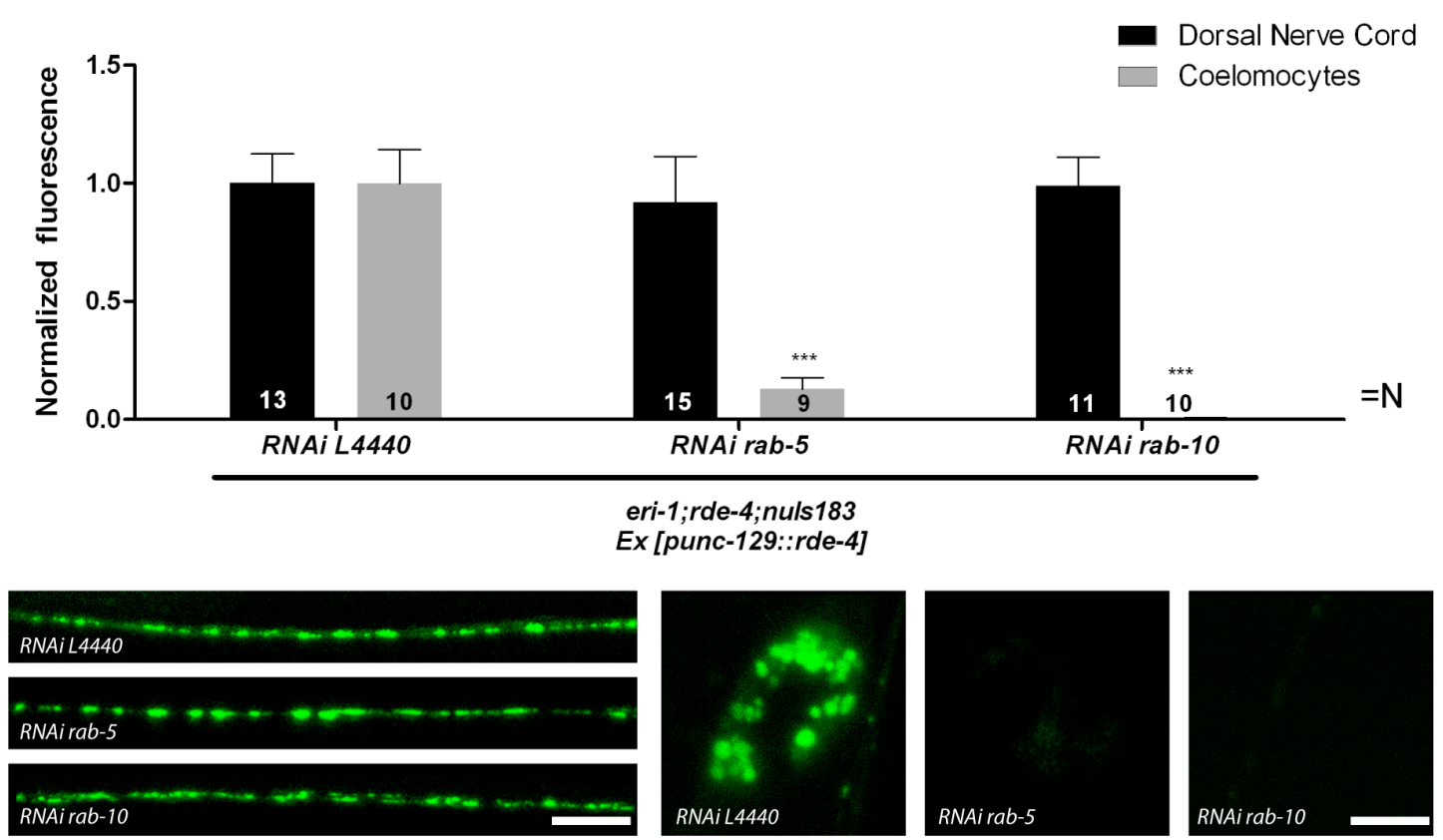

Figure VIII.3. Neuron-specific knockdown of $r \boldsymbol{a b}-5$ and $\boldsymbol{r a b - 1 0}$. Knockdown of $r a b-5$ and $r a b-10$ in the cholinergic motor neurons phenocopied the DCV secretion defect observed in the respective mutants. Scale bar in DNC represents $6 \mu \mathrm{m}$ and bar in coelomocytes is $3 \mu \mathrm{m}$. Error bars $=\mathrm{SEM}(* * *, \mathrm{P}$ $<0.005$; ANOVA with Bonferroni post test)

\subsection{Identification of $\mathrm{RAB}$ regulators involved in $\mathrm{DCV}$ release}

In order to identify novel molecules involved in the regulation of DCV secretion, it was decided to screen regulators of RAB GTPases. Since RAB function is regulated by GEFs and GAPs, we focused on these two families of proteins. At the time of this experiment, more $C$. elegans RAB GAP mutants were available than RAB GEF mutants. Therefore, all neuronally expressed TBC (tre-2/cdc16/Bub2) domain containing RABGAP mutants were screened through the NLP-21-YFP assay (all data not shown). Interestingly, analysis revealed that two Rab GAP mutants displayed similar defects to $r a b-5(Q 78 L)$ and $r a b-10$ mutants. Two mutant alleles of $t b c-2$ were tested: $t b c-2(q \times 20)$ and $t b c-2$ (tm2241) had coelomocyte fluorescent intensities of $28.64 \pm 14.10 \%$ and $18.99 \pm 4.00 \%$, respectively, of wild type. Two mutant alleles of $t b c-4$ were also tested: $t b c-4$ (ok2928) and $t b c-4$ (tm3255) had coelomocyte fluorescent intensities of $5.36 \pm 2.10 \%$ and $4.18 \pm 1.39 \%$ of wild type. DNC intensity levels of these mutants were unchanged (Fig.VIII.4). 


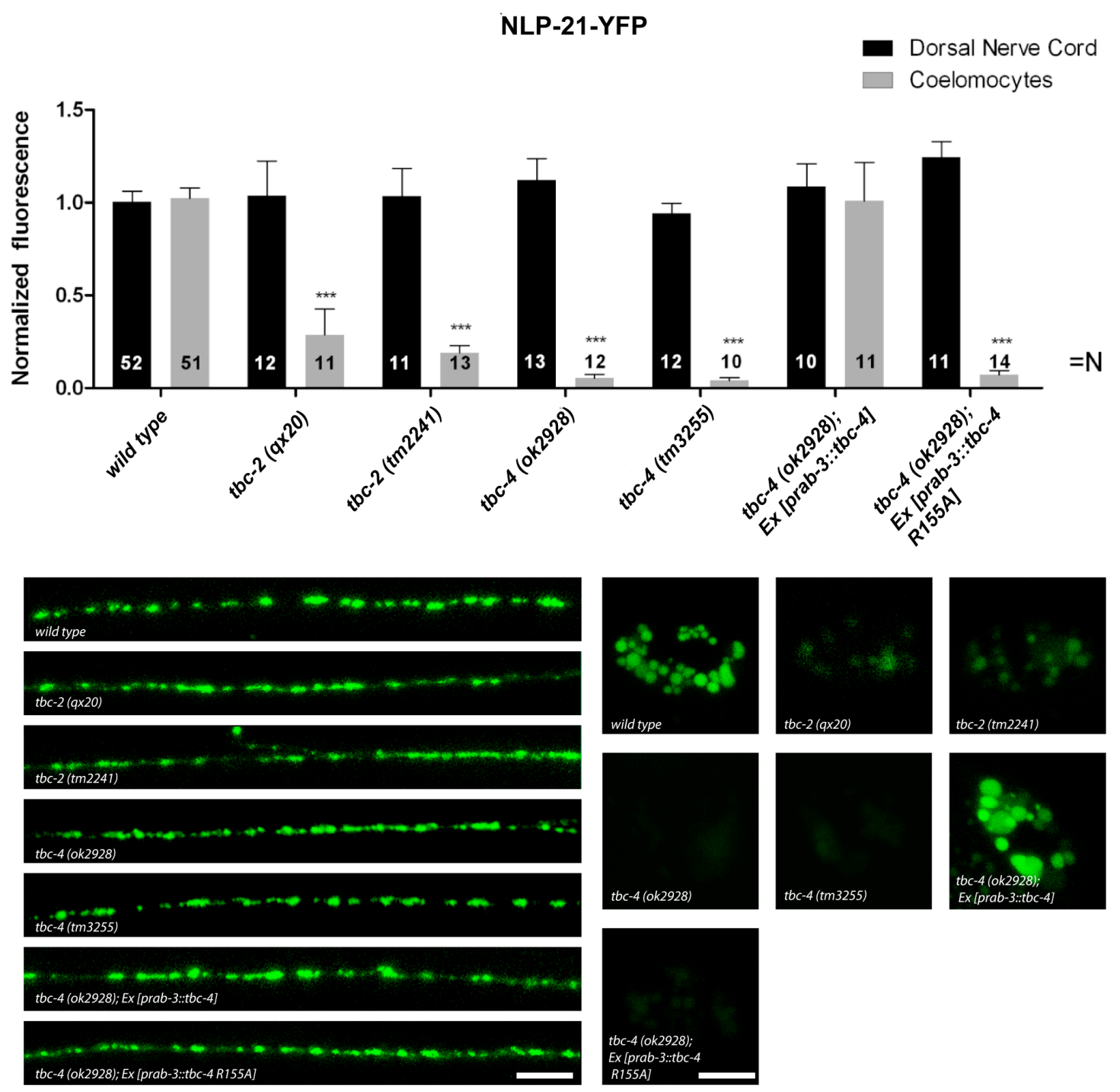

Figure VIII.4. TBC-2 and TBC-4 are novel regulators of DCV secretion. A screen of all mutants containing (Tre-2/Cdc16/Bub2) domains revealed that $t b c-2$ and $t b c-4$ mutants are defective in NLP$21-Y F P$ release. Two different alleles of $t b c-2$ and $t b c-4$ were tested. To demonstrate cell-specificity of the observed phenotype, $t b c-4$ mutants were rescued by expressing $t b c-4$ exclusively in neuron (using the rab-3 promoter). To show that the rescue was dependent on the TBC domain, a catalytically inactive R155A mutant of $t b c-4$ was also tested. Scale bar in DNC represents $6 \mu \mathrm{m}$ and bar in coelomocytes is $3 \mu \mathrm{m}$. Error bars $=\mathrm{SEM}(* * *, \mathrm{P}<0.005$; ANOVA with Bonferroni post test)

To demonstrate that the phenotype of $t b c-4$ mutants was cell-type specific, we conducted neuron-specific (with rab-3 promoter) rescue experiments. Neuron-specific rescue restored coelomocyte fluorescence intensities back to wild type levels. To determine if the regulatory function of TBC-4 was dependent on its catalytic domain, we tested a catalytically inactive mutant, $t b c-4 \mathrm{R} 155 \mathrm{~A}$, for rescue. Interestingly, the $t b c-4$ R155A, was unable to rescue $t b c-4$ mutants suggesting that the role of TBC-4 in DCV secretion is indeed dependent on its ability to regulate the catalytic activity of a RAB (Fig.VIII.4) 


\subsection{RAB-5, TBC-2, RAB-10 and TBC-4 also affect secretion of INS-22}

From the results of the NLP-21-YFP assay it was clear that RAB-5, TBC-2, RAB-10 and TBC-4 are important for the secretion of NLP-21-YFP. However, to rule out the possibility that the observed defect was limited only to NLP-21, we also tested a second DCV marker, INS-22-YFP. In accordance with the NLP-21-YFP results, all four mutants showed decreases in INS-22-YFP in coelomocytes (Fig. VIII.5). rab-5 (Q78L), tbc-2 (tm2241), rab-10 (ok1494) and tbc-4 (ok2928) had coelomocyte fluorescence intensities $48.53 \pm 5.86 \%, 30.10 \pm 8.71 \%, 7.98 \pm 3.16$ $\%$ and $2.82 \pm 0.31 \%$ respectively. It is interesting to note that a trend observed so far is that the $r a b-5$ and the $t b c-2$ mutants display slightly weaker phenotypes than the $r a b-10$ and $t b c-4$ mutants.

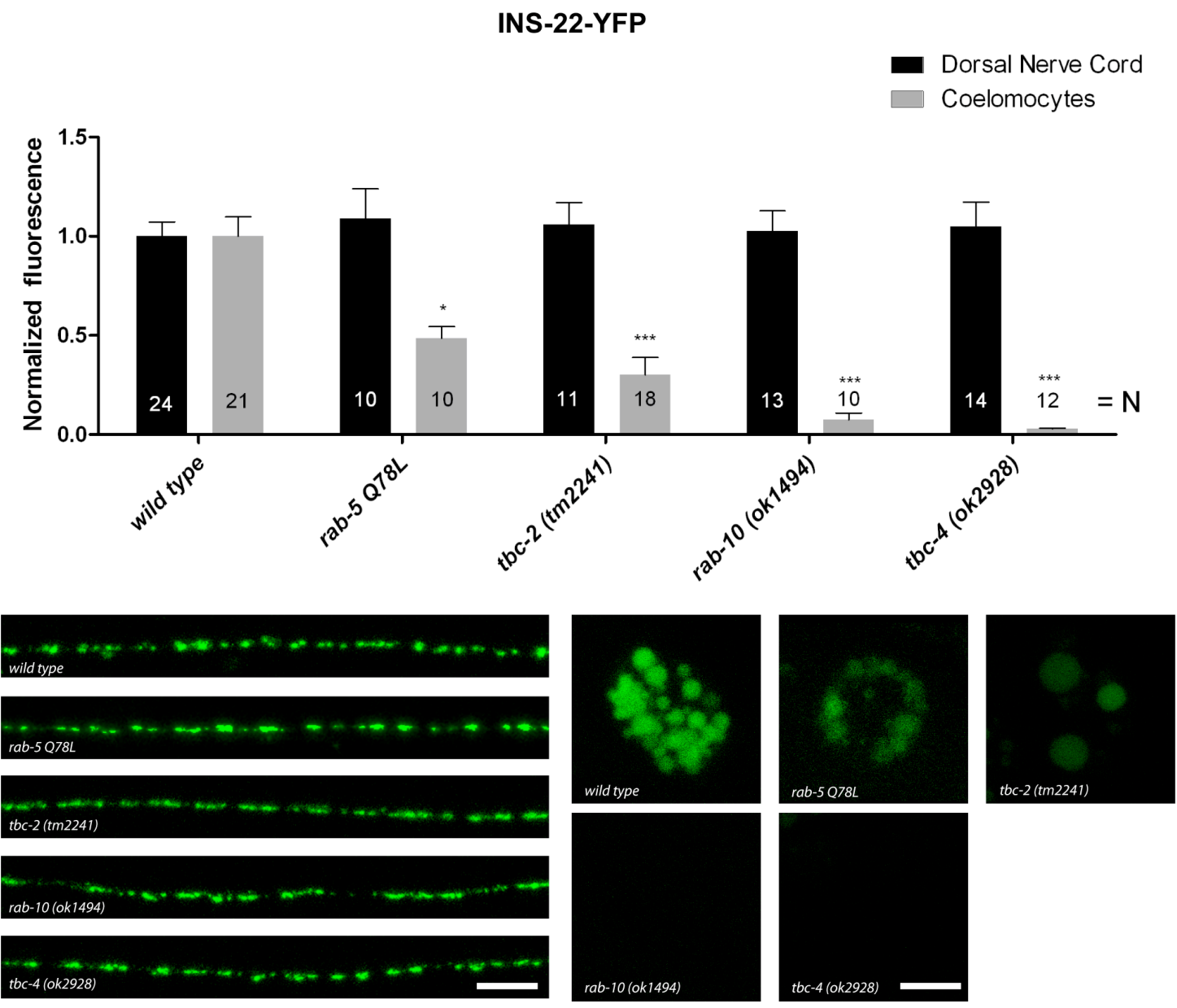

Figure VIII.5. $r a b-5(Q 78 L), t b c-2, r a b-10$ and $t b c-4$ mutants also have defects in INS-22 secretion. In order to verify if the observed defects in DCV secretion were limited to NLP-21-YFP, a second neuropeptide marker, INS-22-YFP was also analyzed. In accordance with the NLP-21-YFP data, mutants of all four genes displayed defects in INS-22-YFP release. Scale bar in DNC represents $6 \mu \mathrm{m}$ and bar in coelomocytes is $3 \mu \mathrm{m}$. Error bars $=$ SEM $(* * *, \mathrm{P}<0.005 ; *, \mathrm{P}<0.01$ ANOVA with Bonferroni post test) 


\subsection{Endocytosis in coelomocytes is not affected}

Both the NLP-21-YFP and INS-22-YFP assays rely on coelomocyte endocytosis of YFP as reporter. To ensure that the observed defects were indeed due to altered DCV release and not due to altered coelomocyte endocytosis, we examined the integrity of coelomocyte endocytosis. This was tested through the injection of soluble Texas-Red conjugated to bovine serum albumin (TR-BSA), into the worm body cavity. In wild type animals, the TR-BSA is readily endocytosed by the coelomocytes and enters early endosomes (labeled with RME-8-GFP marker) within the first 5 minutes post injection. The dye then starts to migrate out of the RME-8GFP positive endosomes and onto late endosomes after 30 minutes. Usually after 4550 minutes almost all of the TR-BSA is found in the late endosomes. rab-5 (Q78L), $t b c-2$ (tm2241), rab-10 (ok1494) and tbc-4 (ok2928) were all tested by TR-BSA injection and results showed that the ability of these mutants to endocytose and the kinetics of endocytosis were unaltered (Fig. VIII.6).

\subsection{Synaptic vesicle release is not affected}

\subsubsection{Analysis of SV markers: SNB-1 and RAB-3}

The TR-BSA endocytosis assay signified that RAB-5, TBC-2, RAB-10 and TBC-4 are factors required for DCV secretion. Since, it has been shown that much of the DCV secretion machinery is also necessary for SV release, we verified if our molecules of interest were also important for SV exocytosis. This was first studied by imaging two different SV markers: GFP-Synaptobrevin and YFP-RAB-3. These markers were expressed in the cholinergic motor neurons (DA and DB). In the neurons, both markers localize to SVs at the DNC synapse and can be viewed as discrete puncta. Each puncta is thought to correspond to the presynapse of a neuromuscular synapse (Sieburth et al., 2005). Fluorescence intensities of puncta, number of puncta and puncta size of GFP-SNB-1 and YFP-RAB-3 were measured for $r a b-5$ (Q78L), tbc-2, rab-10 and tbc-4 mutants. Interestingly, analysis of both SV markers revealed no significant changes suggesting that SVs were localized correctly and were unaffected (Fig VIII.7). 

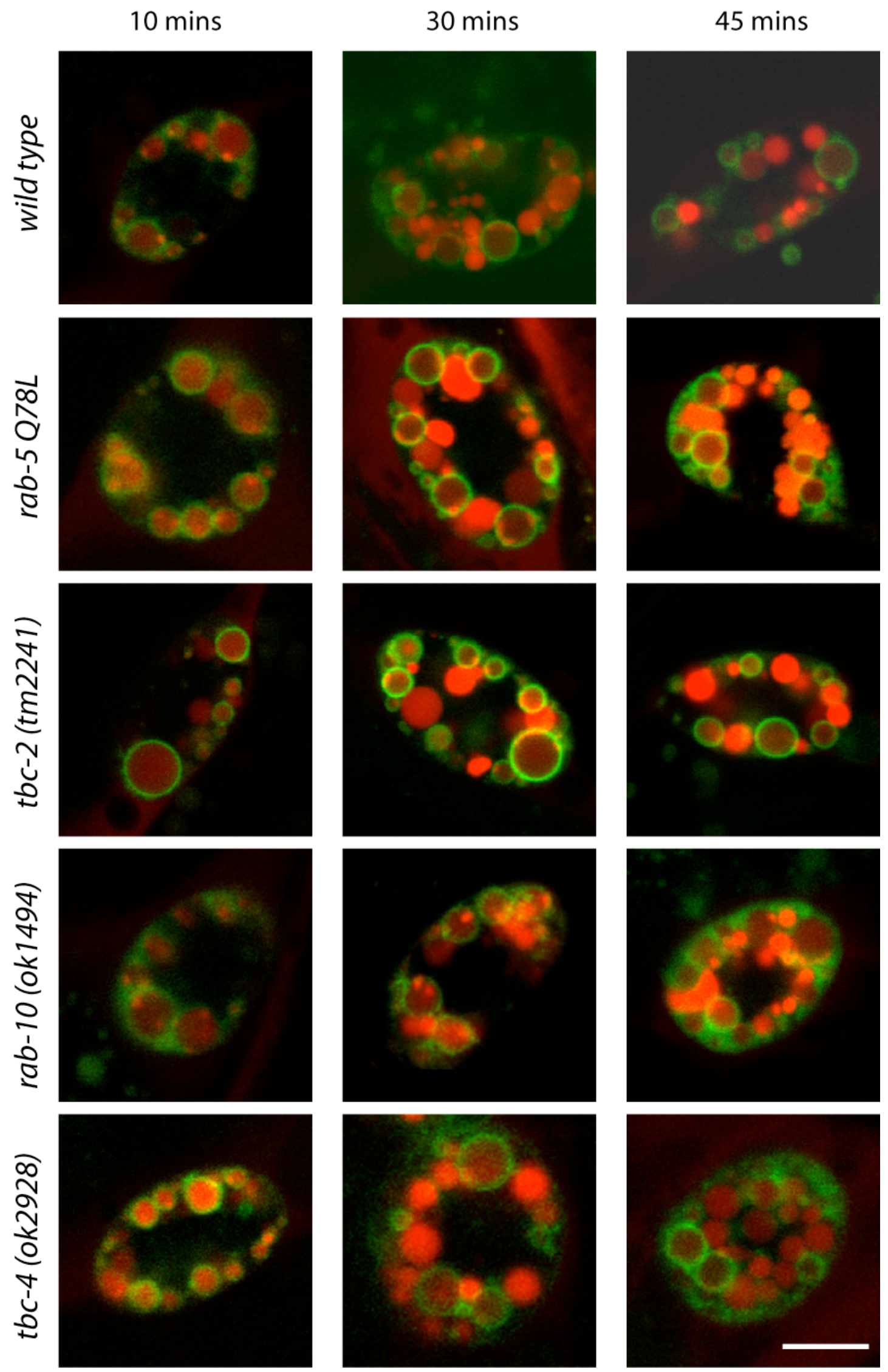

Figure VIII.6. Assessment of coelomocyte function through TR-BSA injection. Analysis of $r a b-5$ $(Q 78 L), t b c-2, r a b-10$ and $t b c-4$ mutants to endocytose TR-BSA showed that these mutants had no defects in the ability to endocytose or in the kinetics of endocytosis. RME-8-GFP was used as an early endosomal marker. TR-BSA (Red) and RME-8-GFP (Green). Scale bar represents $5 \mu \mathrm{m}$. 
A
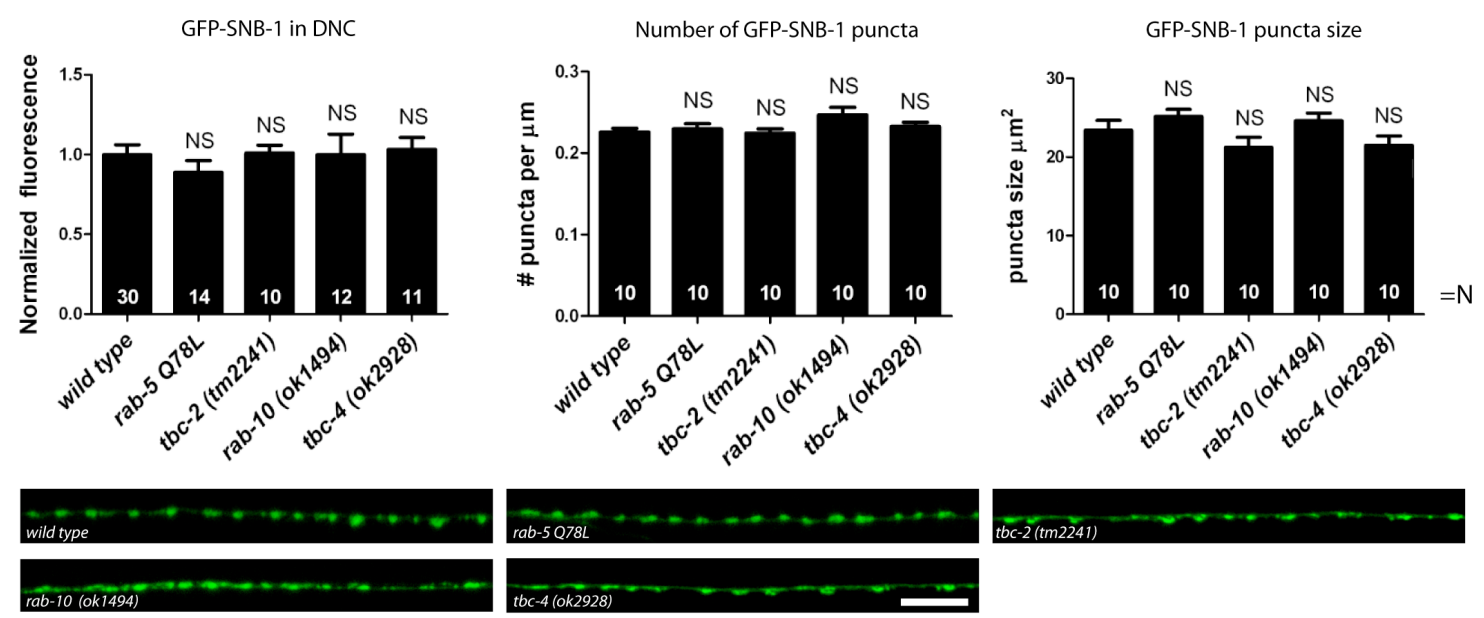

B
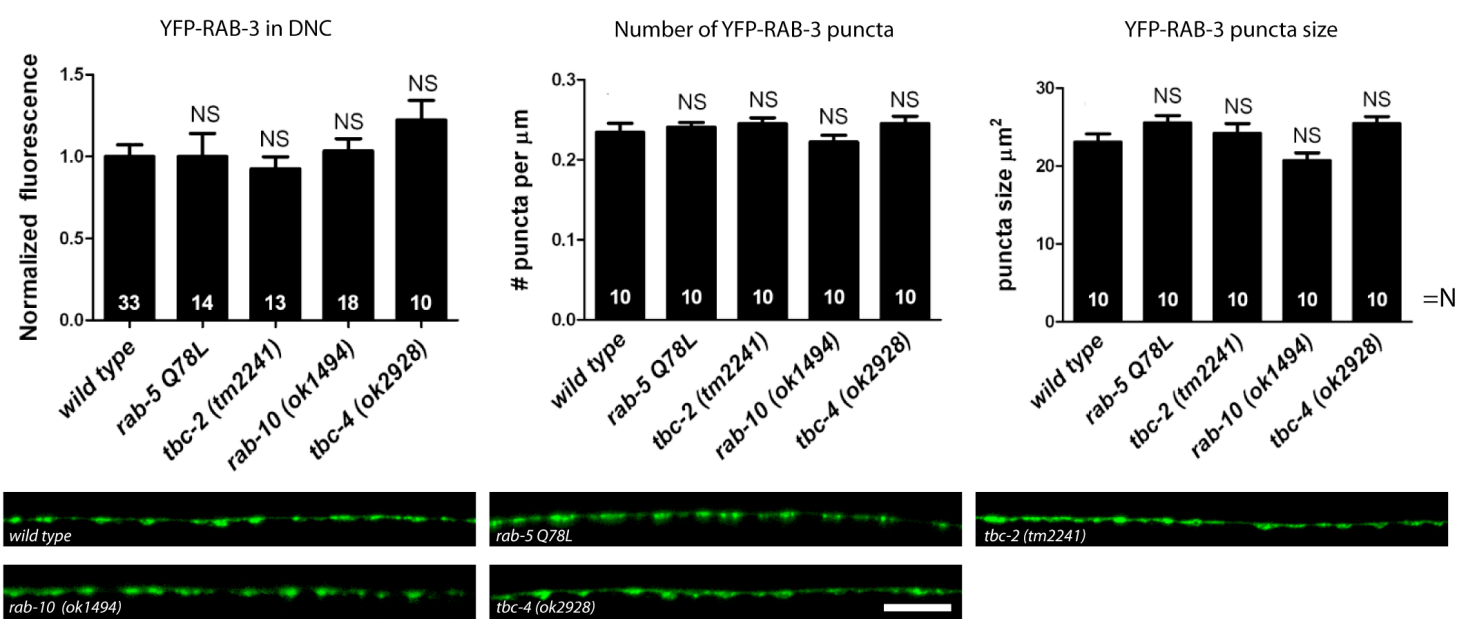

Figure VIII.7. Analysis of SV markers. A. GFP-SNB-1 fluorescence levels were unchanged in $r a b-5$ (Q78L), $t b c-2, r a b-10$ and $t b c-4$ mutants. Additionally, the number and size of GFP-SNB-1 puncta were also unaffected in these mutants. B. YFP-RAB-3 fluorescence levels were unchanged in $r a b-5$ $(Q 78 L), t b c-2, r a b-10$ and $t b c-4$ mutants. Additionally, the number and size of YFP-RAB-3 puncta were also unaffected in these mutants. Scale bar in DNC represents $6 \mu \mathrm{m}$. Error bars $=$ SEM (ANOVA with Bonferroni post test).

\subsubsection{EM analysis of $\mathrm{SV}$ distributions in synapses}

The analysis of GFP-SNB-1 and YFP-RAB-3 markers was limited to the resolution of the confocal microscope. In order to assess, at high resolution, the integrity of synapses, we analyzed $r a b-5$ (Q78L), tbc-2, $r a b-10$ and $t b c-4$ mutants using high-pressure freeze electron microscopy (HPF-EM). An overall morphological observation of mutant synapses revealed that mutant synapses were largely similar to wild type (Fig. VIII.8). Some rab mutants, such as $r a b-3$, have been shown to display altered SV distributions in synapses as a result of a tethering defect. In order to determine if $r a b-5, r a b-10, t b c-2$ and $t b c-4$ mutants had similar defects in tethering, 
A

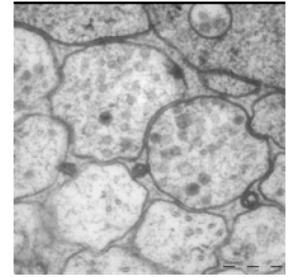

wild type

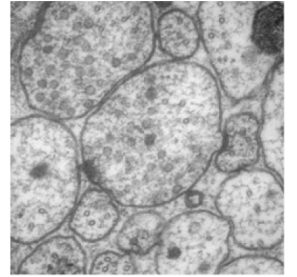

rab-5 Q78L

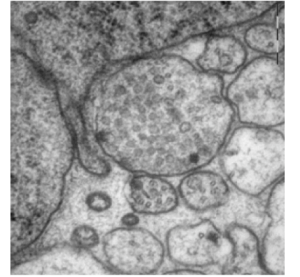

tbc-2 ( $q \times 20)$

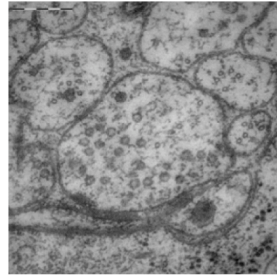

rab-10 (ok1494)

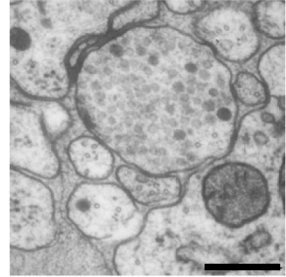

tbc-4 (ok 2928)

B

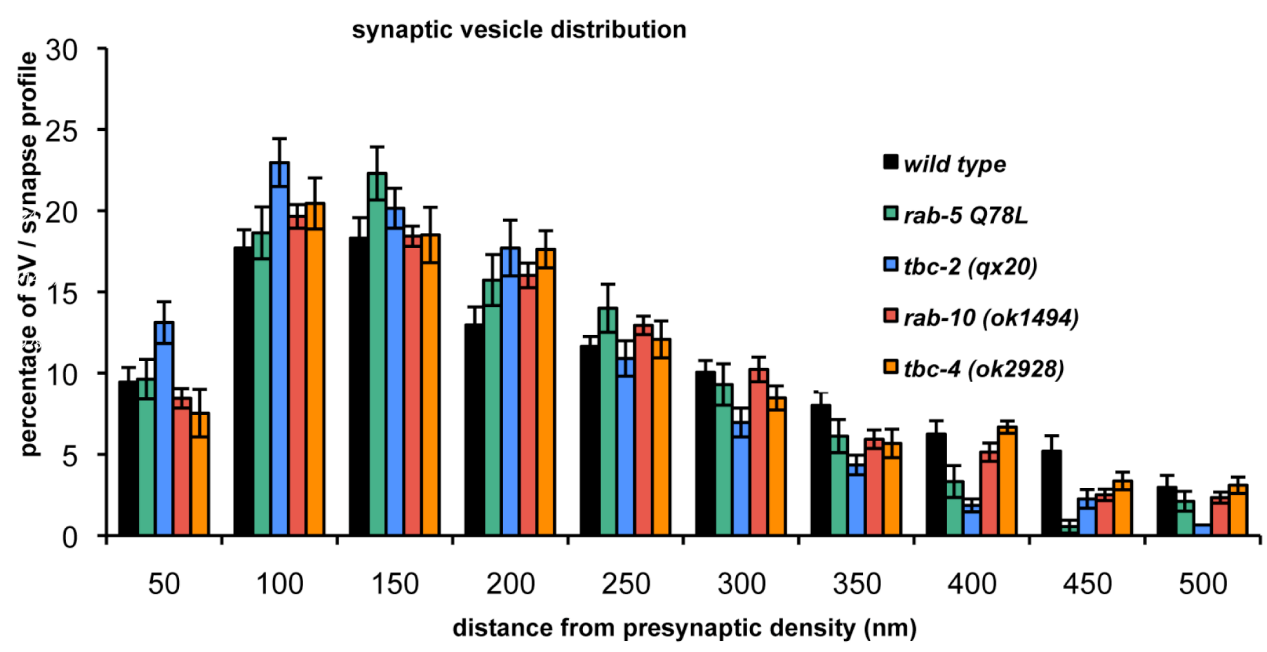

\begin{tabular}{lcccccc}
\hline Genotype & $\begin{array}{c}\text { Number of } \\
\text { profiles / animal } \\
\text { analyzed }\end{array}$ & $\begin{array}{c}\text { Mean synaptic } \\
\text { area }\end{array}$ & SV / profile & $\begin{array}{c}\text { Mean diameter } \\
\text { of SV }\end{array}$ & $\begin{array}{c}\text { DCV / profile } \\
\text { Mean diameter } \\
\text { of DCV }\end{array}$ \\
\hline wild type & $80 / 8$ & $0,21 \pm 0,01$ & $33,7 \pm 1,6$ & $31,2 \pm 0,4$ & $2,1 \pm 0,2$ & $44,3 \pm 0,7$ \\
rab-5 $Q 78 L$ & $47 / 3$ & $0,18 \pm 0,01$ & $30,1 \pm 1,5$ & $30,7 \pm 0,4$ & $2,3 \pm 0,5$ & $44,9 \pm 0,9$ \\
$t b c-2$ (qx20) & $45 / 3$ & $0,17 \pm 0,01$ & $39,7 \pm 2,1$ & $31,5 \pm 0,5$ & $2,4 \pm 0,3$ & $43,4 \pm 0,7$ \\
rab-10 (ok1494) & $51 / 7$ & $0,23 \pm 0,04$ & $31,2 \pm 1,9$ & $29,3 \pm 0,3$ & $1,9 \pm 0,2$ & $43,5 \pm 0,4$ \\
$t b c-4$ (ok2928) & $44 / 3$ & $0,19 \pm 0,01$ & $31,5 \pm 1,4$ & $31,7 \pm 0,4$ & $2,0 \pm 0,3$ & $44,0 \pm 0,7$ \\
\hline
\end{tabular}

Figure VIII.8. HPF-EM analysis of synapses. A. Morphological analysis of $r a b-5(Q 78 L), t b c-2$, $r a b-10$ and $t b c-4$ mutant synapses appeared similar to wild type. Scale bar represents $400 \mathrm{~nm}$. B. Analysis of SV distributions in mutants showed that they are unaffected. Error bars = SEM (ANOVA with Bonferroni post test). Source: This experiment was conducted by Christian Olendrowitz and Jan Hegermann. The original pictures and data were kindly provided by them.

we analyzed the distribution of SVs from the active zone in the mutant synapses. Statistical analysis showed that SV distributions were also unaffected (Fig. VIII.8). From the same synapses, the number and size of DCVs were also analyzed and appeared like wild type. 


\subsubsection{EM analysis of neuron morphology}

After confirming that the neuronal synapses and the SV distributions were normal, we analyzed the neuronal cell bodies of $r a b-5$ (Q78L), tbc-2, $r a b-10$ and $t b c-$ 4 mutants to verify if there were any upstream changes, which may affect nervous system function. Neuronal cell somas and neuronal Golgis were analyzed by HPFEM for each mutant. Results revealed that all four mutants had no obvious abnormalities in neuronal morphology (Fig. VIII.9).

\subsubsection{Electrophysiological analysis of synaptic transmission}

Both confocal and EM data showed that there were no defects in SV localizations, synapse morphology and neuron morphology. In order to determine if there was any changes in SV exocytosis, we measured excitatory postsynaptic currents (EPSCs) at the NMJs by whole cell patch clamp. This experiment was done in collaboration with Alexander Gottschalk's lab, Johann Wolfgang Goethe University in Frankfurt. All $r a b-5$ (Q78L), $t b c-2, r a b-10$ and $t b c-4$ mutants were crossed into a strain expressing channelrhodopsin-2 in the cholinergic motor neurons. Photo-evoked responses revealed that there were no changes in EPSCs (Fig. VIII.10). These results indicated that $r a b-5(Q 78 L), t b c-2, r a b-10$ and $t b c-4$ mutants are most likely factors involved in DCV release and not SV exocytosis.

\subsection{TBC-4 is a potential GAP for RAB-10}

Considering the strong similarity in phenotypes between $r a b-5(Q 78 L), t b c-2$, $r a b-10$ and $t b c-4$ mutants, an obvious interpretation is that TBC-2 and TBC-4 may be GAPS for RAB-5 and RAB-10. In line with this, a very recent study showed that TBC-2 is indeed the GAP for RAB-5 (Chotard et al., 2010). This was revealed through an in vitro GAP assay with recombinant RAB-5 and TBC-2 purified from E.coli. These results led us to believe that TBC-4 could potentially be the GAP for RAB-10. Although, it was possible to purify TBC-4, attempts to recombinantly purify RAB-10 in E.coli failed. Thus, in the meantime, we attempted a yeast-2-hybrid (Y2H) strategy to show the substrate specificity of TBC-4. Since RAB-GAP interactions are in the order of $200 \mu \mathrm{M}$ (personal communication with Aymelt Itzen, MPI for Molecular Physiology), it is difficult to detect such transient interactions. A previous study by Francis Barr's lab showed that RAB-GAP interactions could be 

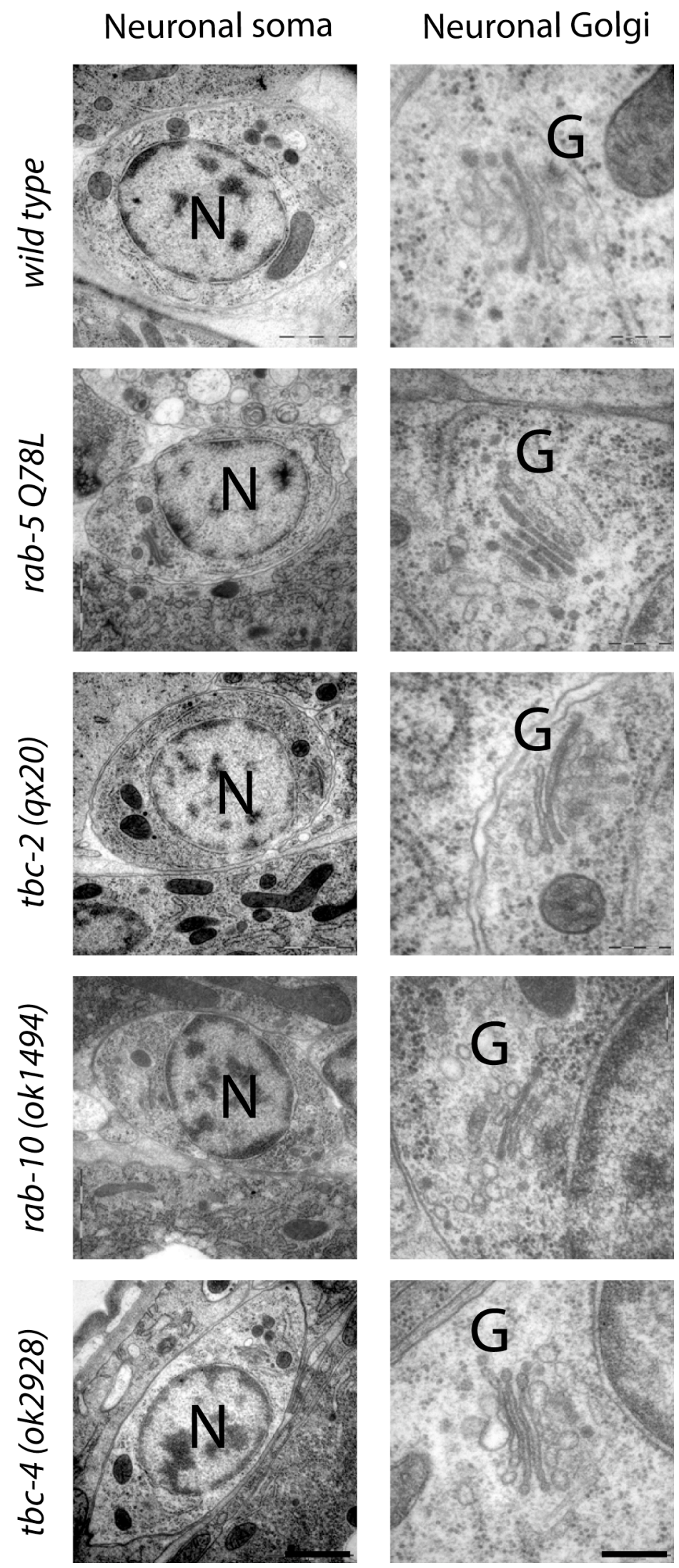

Figure VIII.9. HPF-EM analysis of neuron morphology. Morphological analysis of $r a b-5(Q 78 L)$, $t b c-2, r a b-10$ and $t b c-4$ mutant neurons and their Golgis revealed that were no obvious defects. N: Nucleus and G: Golgi. Scale bar represents $500 \mathrm{~nm}$ in somas and $200 \mathrm{~nm}$ in Golgis. Source: This experiment was conducted by Jan Hegermann and the images were kindly provided by him. 

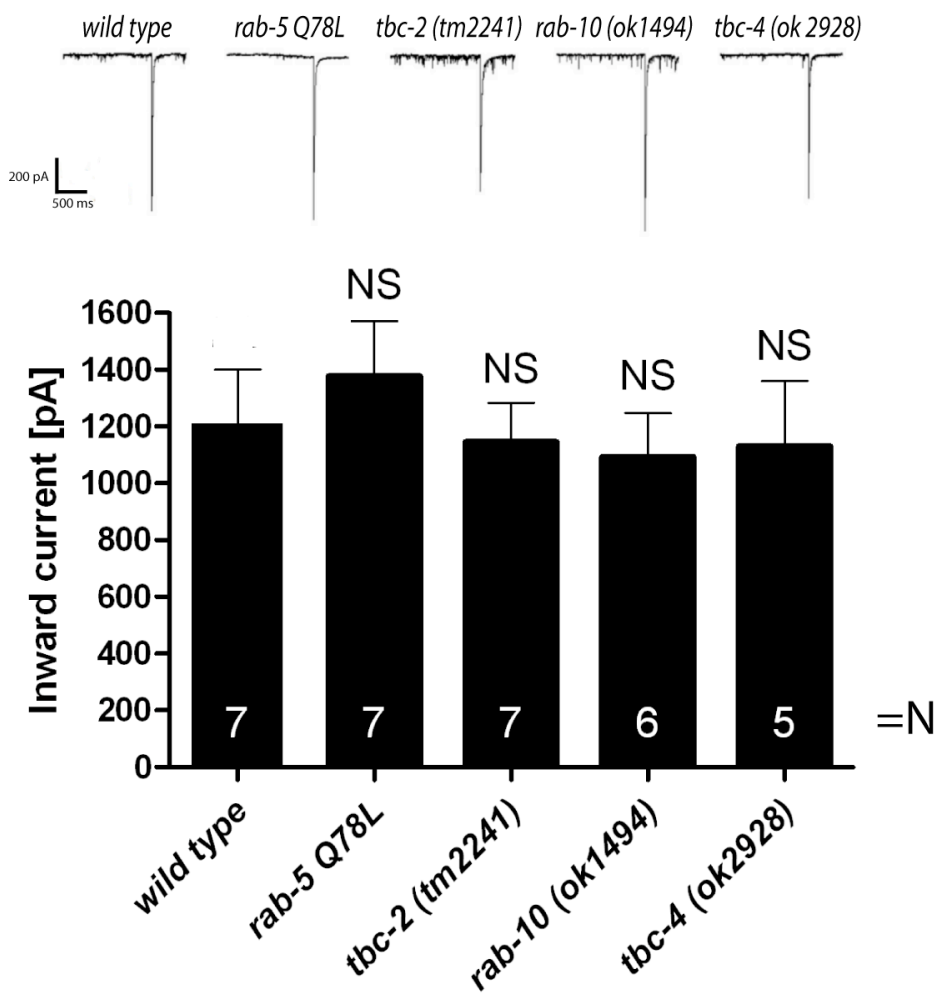

Figure VIII.10. Electrophysiological analysis of synaptic function. $r a b-5$ (Q78L), $t b c-2, r a b-10$ and $t b c-4$ mutants were crossed into a strain expressing channelrhodopsin-2 in the cholinergic motor neurons. Photo-evoked responses from whole cell patch clamp experiments of NMJs showed that evoked EPSCs were normal in all four mutants. Error bars = SEM (ANOVA with Bonferroni post test). Source: This experiment was conducted by a member of Alexander Gottschalk`s lab, Johann Wolfgang Goethe University in Frankfurt. The original data was kindly provided by them.

detected through $\mathrm{Y} 2 \mathrm{H}$ (Haas et al., 2005). However, for capturing such interactions the catalytic arginine residue of the GAP must be mutated to alanine. This enabled Haas et al., to show that Rabgap-5 is the GAP for Rab5 in mammals. We chose to use a similar approach to test TBC-4 and RAB-10. The TBC domain of TBC-4 is between residues 106 and 316 (SMART prediction). Additionally it contains four coiled coil (CC) domains (please see appendix for full protein sequence). The catalytic R155 was mutated to A and tested against a constitutively active RAB-10 (Q68L) as well as other C. elegans RABs. Surprisingly, results showed that RAB-10 binds to TBC-4 (R155A) in a GTP-dependent manner because the constitutively inactive RAB-10 (T23N) did not bind to TBC-4 (R155A) (Fig. VIII.11). This provided evidence that TBC-4 may indeed be a RAB-10 specific GAP. Through our interaction analysis we also found that TBC-4 binds two additional RABs: RAB-8 and RAB-11.1. Since these interactions were found also in the wild type TBC-4 and the TBC-4 R155A, it is likely that these RABs are binding to sites outside the TBC domain (Fig. VIII.11). 
A

TBC-4:

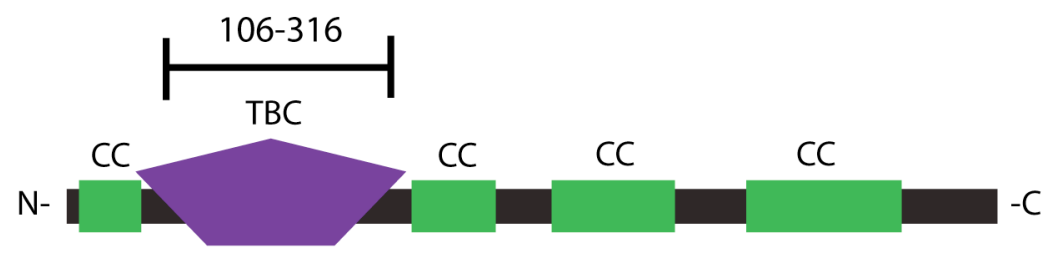

B

TBC-4
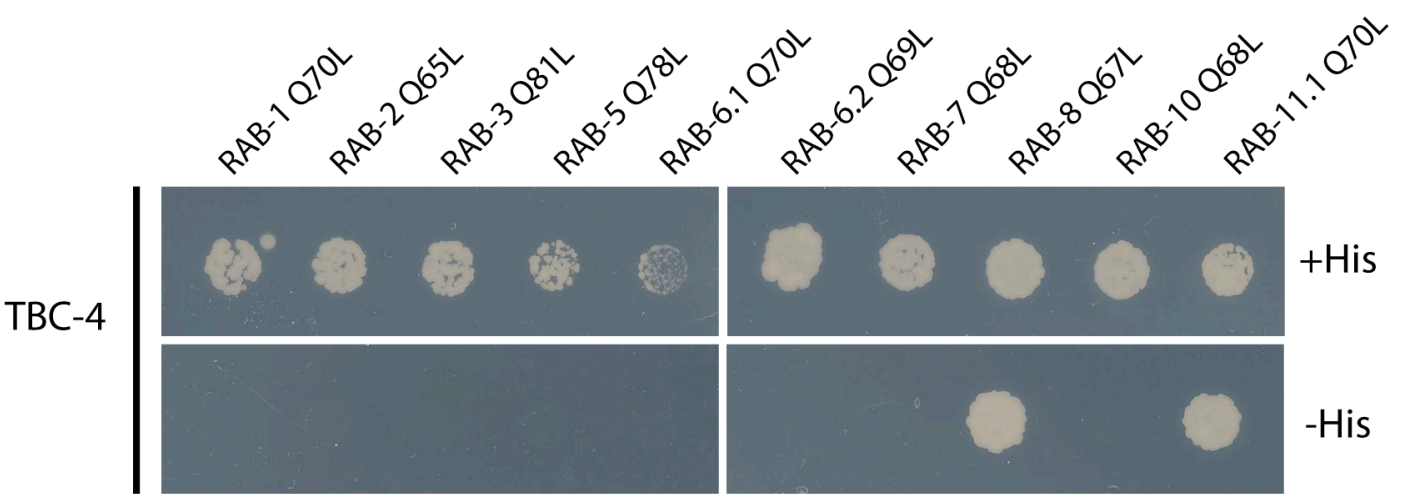

$+\mathrm{His}$

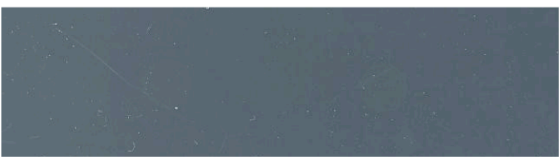

-His
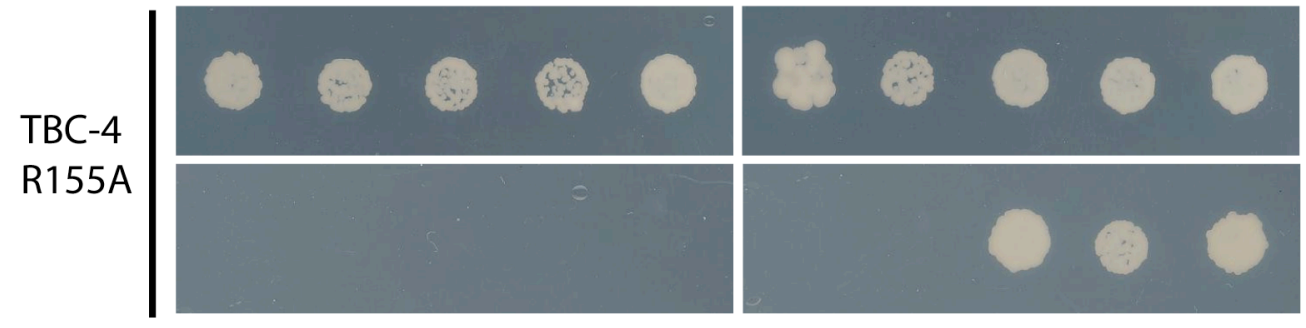

$+\mathrm{His}$

C

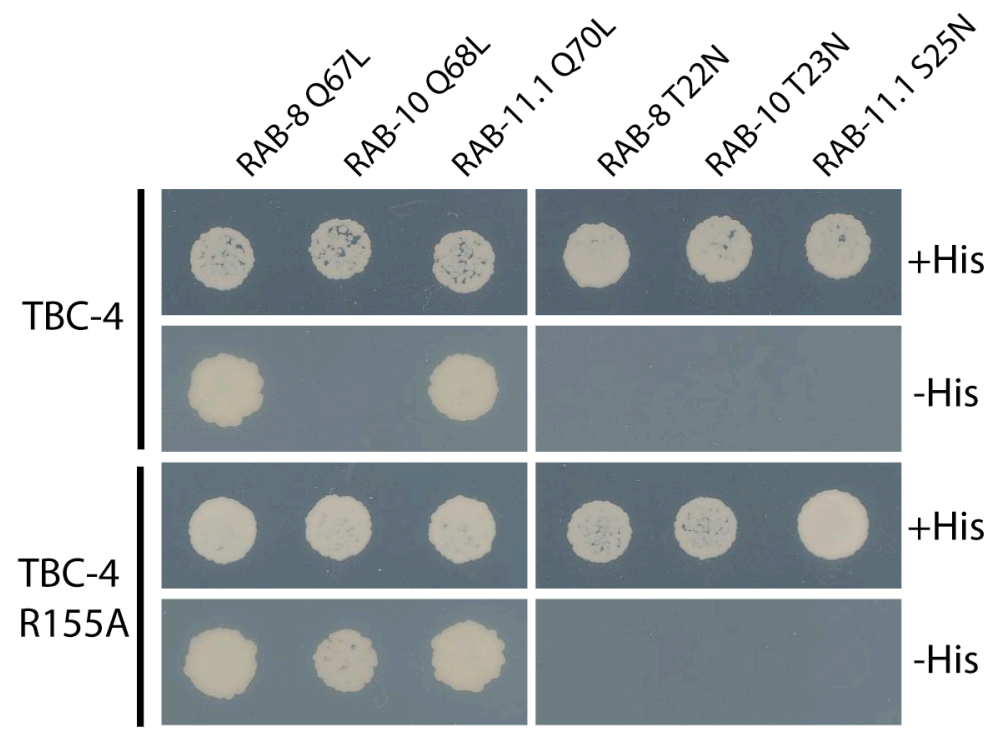

Figure VIII.11. Y2H analysis of TBC-4 binding. A. TBC-4 is a 825 aa protein, which contains multiple domains. The TBC domain (purple) is predicted to be between 106 to 316 aa. Additionally there are 4 coiled coil (CC) domains. Please see appendix for protein sequence. B. Y2H analysis showed that TBC-4 binds to RAB-8, RAB-10 and RAB-11.1. Strikingly the interaction with RAB-10 is dependent on the R155A mutation. C. The binding to TBC-4 was abolished in constitutively inactive RABs. Source: This experiment was conducted by the author and Mandy Hannemann. 
Preliminary data suggests that they are binding to the N-terminal $\mathrm{CC}$ domain, which lies upstream of the TBC domain (data not shown).

\subsection{Rabaptin-5 bridges RAB-5 and RAB-10 function}

Since the phenotypes of RAB-5 and TBC-2 were surprisingly similar to RAB5 and TBC-4, we hypothesized they belonged to the same pathway and function together to regulate DCV release. To answer this, we screened with $\mathrm{Y} 2 \mathrm{H}$ all known RAB-5 effectors against RAB-10 and TBC-4 to identify if there were any common molecules. To our surprise, we found that Rabaptin-5 (RABN-5), a RAB-5 effector shown to be important in endosomal fusion (Stenmark et al., 1995), interacted with TBC-4. After testing different domains of TBC-4, it was identified that RABN-5 interacted with the C-terminal $\mathrm{CC}$ domains downstream of the TBC-domain (Fig. VIII.12).

TBC-4:

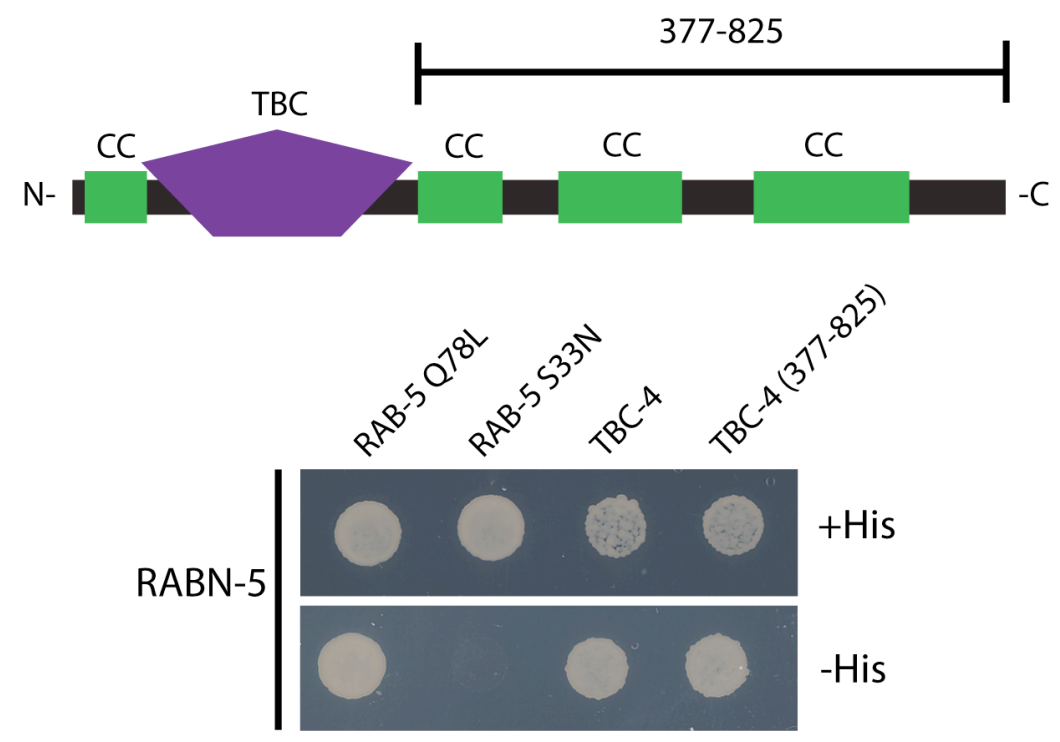

Figure VIII.12. Y2H analysis of TBC-4 interaction with RABN-5. TBC-4 interacts with RAB-10 in a GTP dependent manner. Additionally the interaction occurs at the $\mathrm{C}$-terminal coiled coil domains (377-825) of TBC-4. Source: This experiment was conducted by Mandy Hannemann and the data was kindly provided by her.

The Y2H interaction of RABN-5 with TBC-4 suggested that there may be a RAB cascade between RAB-5 and RAB-10 that is necessary for DCV function. Furthermore, RABN-5 would act as a bridging molecule between these two RABs. To verify if RABN-5 was also a regulator of DCV release, we tested the effect of RABN-5 knockdown on NLP-21-YFP secretion. Since the rabn-5 locus is on the 
same chromosome and in close proximity to the NLP-21-YFP insertion, we could not test the mutant. Therefore, we relied on neuron-specific RNAi. Interestingly, knockdown of rabn-5 led to a decrease in NLP-21 secretion. Fluorescence intensity values were $32.75 \pm 14.80 \%$ compared to knockdown of mock, L4440 secretion (Fig. VIII.13). Intensity levels in the DNC were unchanged. Since RABN-5 has also been shown to be involved in the endocytic pathway (Stenmark et al., 1995), we wanted to verify if the observed defect of RABN-5 on NLP-21-YFP release was due to perturbation of endocytosis. Therefore, another molecule involved in early endocytic transport, early endosomal antigen-1 (EEA-1) (Simonsen et al., 1998), was knocked down by RNAi and analyzed for NLP-21-YFP secretion (Fig. VIII.13). Interestingly depletion of EEA-1 showed no defect, suggesting that the observed role of RABN-5 in DCV secretion is a novel secondary role of this protein. To confirm this we also tested rabn-5 mutants in the INS-22-YFP secretion assay. In accordance with the NLP-21-YFP data, rabn-5 mutants had coelomocyte intensities of $43.13 \pm 3.71 \%$ of wild type and normal levels of INS-22-YFP in the DNC (data not shown).
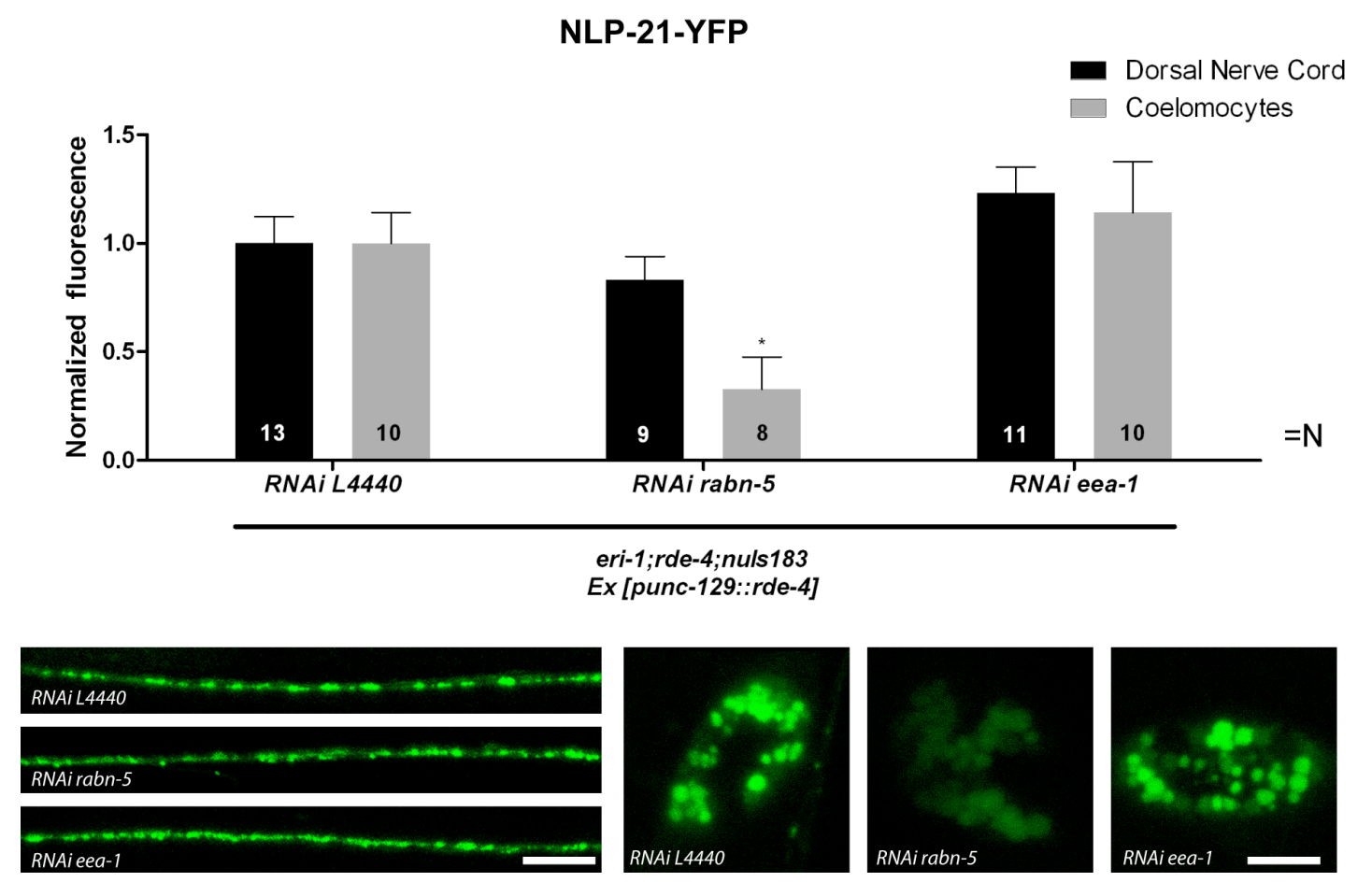

Figure VIII.13. NLP-21-YFP analysis of RABN-5 and EEA-1. Neuron specific RNAi of rabn-5 showed a decrease in coelomocyte intensity levels suggesting a role in DCV release. Knockdown of another RAB-5 effector, eea-1, displayed no defect in DCV secretion. Scale bar in DNC represents 6 $\mu \mathrm{m}$ and bar in coelomocytes is $3 \mu \mathrm{m}$. Error bars $=\mathrm{SEM}(*, \mathrm{P}<0.05$; ANOVA with Bonferroni post test) 


\subsection{EHBP-1 also regulates DCV release and interacts with RAB-10}

During the course of this study, one of our collaborators had identified a novel RAB-10 effector in C. elegans, named EHBP-1 (Personal communication with Barth Grant, Rutger's University, New Jersey). EHBP-1 contains a calponin homology $(\mathrm{CH})$ domain and a $\mathrm{CC}$ domain. Firstly we verified the specificity of the RAB-10EHBP-1 interaction, by Y2H screening of EHBP-1 against all C. elegans RABs. The full length EHBP-1 was tested as well as a shorter fragment containing only the CC domain. Interestingly, the full length EHBP-1 did not interact with any RABs and the shorter EHBP-1(662-901) interacted with RAB-8 and RAB-10 (Fig. VIII.14).

To determine if EHBP-1 is also involved together with RAB-10 in DCV secretion, we tested it through the NLP-21-YFP assay. The ehbp-1 mutant is lethal, so we chose to test it through the neuron-specific RNAi strain. Results revealed that knockdown of ehbp-1 leads to a severe defect in DCV release (Fig. VIII.14). The fluorescence intensity in coelomocytes was $1.00 \pm 0.20 \%$ of the mock knockdown. This suggested that EHBP-1 is also involved in this pathway.

\subsection{Colocalization analysis of novel factors involved in DCV release}

Six different molecules were identified in this study to participate specifically in DCV secretion: RAB-5, TBC-2, RAB-10, TBC-4, RABN-5 and EHBP-1. To determine where these molecules function in the cell, a sub-cellular localization analysis was done. Proteins of interest were tagged with fluorescent proteins and coinjected with compartmental markers. Since, the localization of RAB-5 and TBC-2 to the endo-lysosomal system has already been well characterized in C. elegans (Chotard et al., 2010), we started by focusing on RAB-10. The localization of RAB10 with respect to ER (CB5-GFP), COPI coat (GFP-عCOP), Golgi (MANNSII-YFP), Endosomes (GFP-2xFYVE), iDCVs/endosomes (tagRFP-SYX-6) and synapses (GFP-SNB-1 and YFP-RAB-3) was verified (Fig. VIII.15). Co-localization analysis revealed that RAB-10 puncta are detectable in the neuronal cell soma and at the synapse. In the soma, RAB-10 showed a partial overlap with Golgi and endosomal markers. At the synapses, RAB-10 showed almost full colocalization with SNB-1 and RAB-3. However, the SV markers appeared more concentrated and brighter whereas RAB-10 staining was weaker and diffuse. This suggests that RAB-10 could 
A EHBP-1:

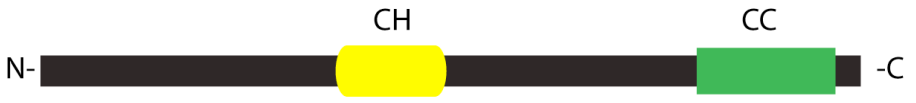

$+\mathrm{His}$

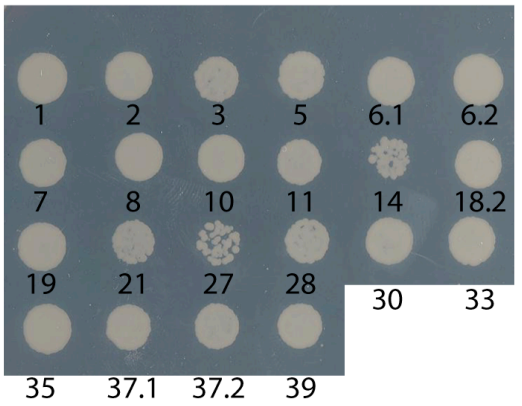

EHBP-1 (662-901):
-His

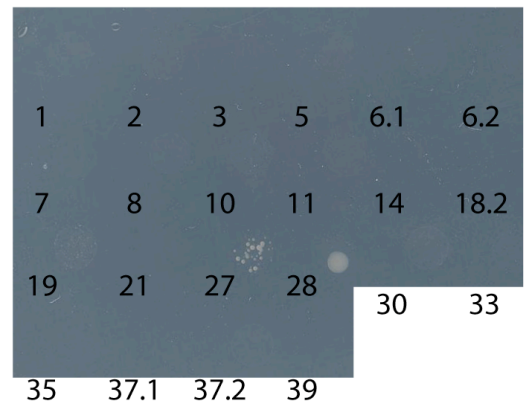

$\mathrm{N}-$

$+\mathrm{His}$

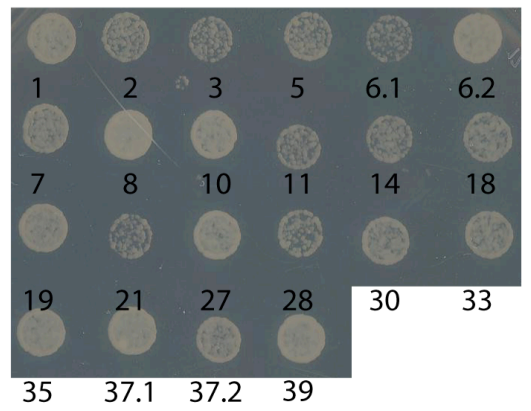

B

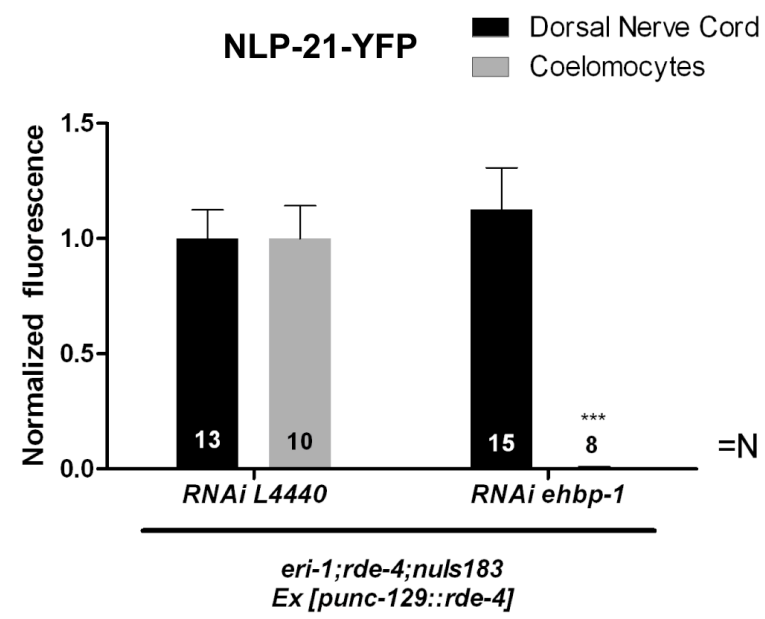

$-\mathrm{His}$

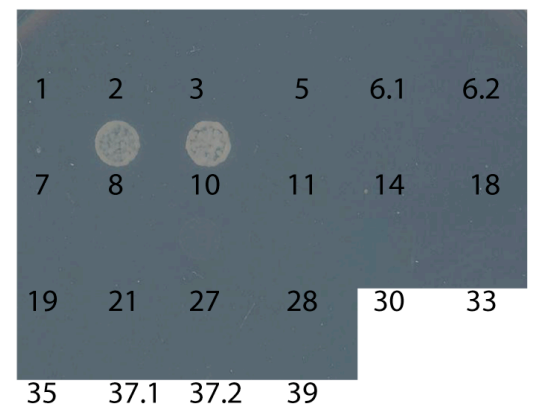

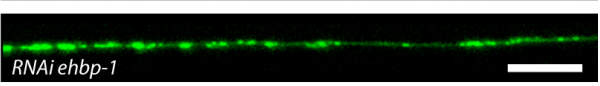
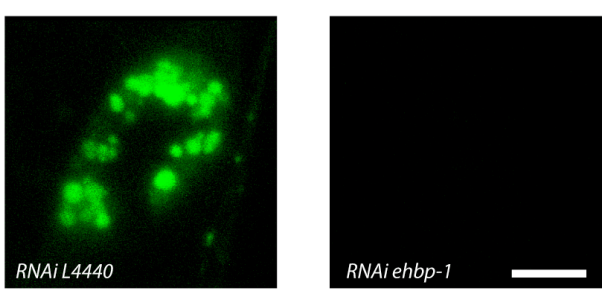

RNAiehbp-1

Figure VIII.14. Y2H and NLP-21-YFP analysis of a RAB-10 effector. A. The full length EHBP-1 did not interact with any Rabs. A shorter fragment, which included the CC domain (662-901), interacted with RAB-10 and RAB-8. B. Neuron specific RNAi of $e h b p-1$ showed a decrease in coelomocyte intensity levels. Scale bar in DNC represents $6 \mu \mathrm{m}$ and bar in coelomocytes is $2.5 \mu \mathrm{m}$. Error bars $=$ SEM $(* * *, \mathrm{P}<0.005$; ANOVA with Bonferroni post test) 

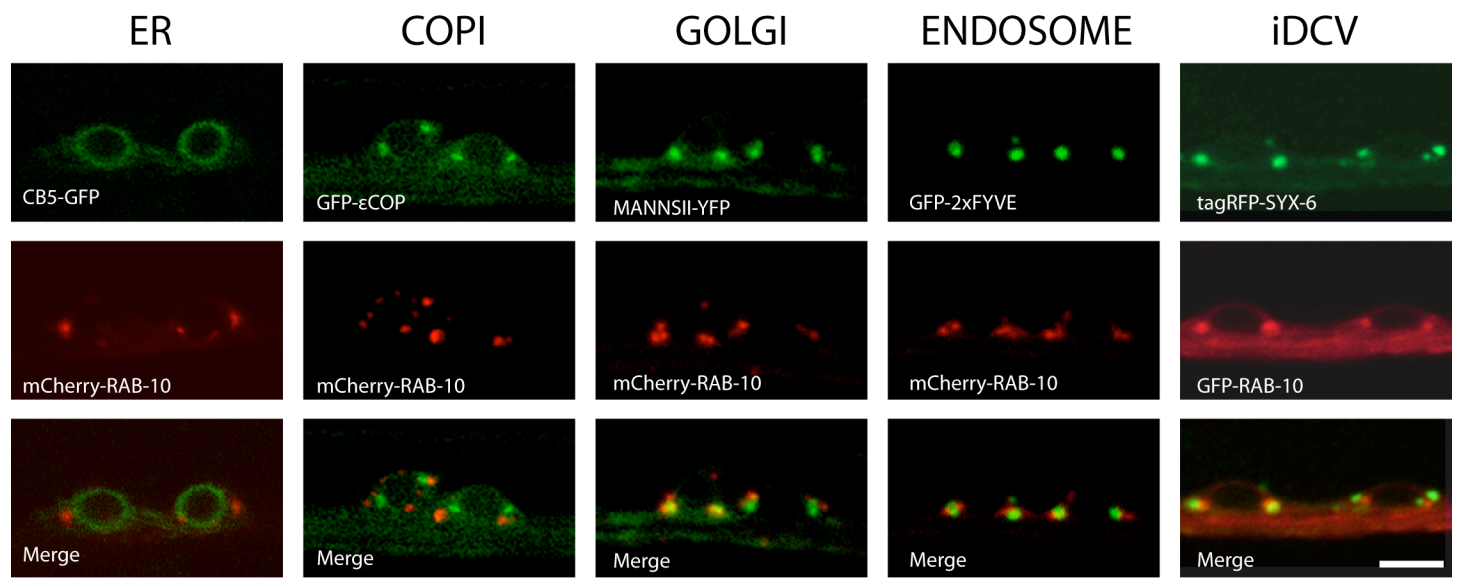

SYNAPSE I

SYNAPSE II
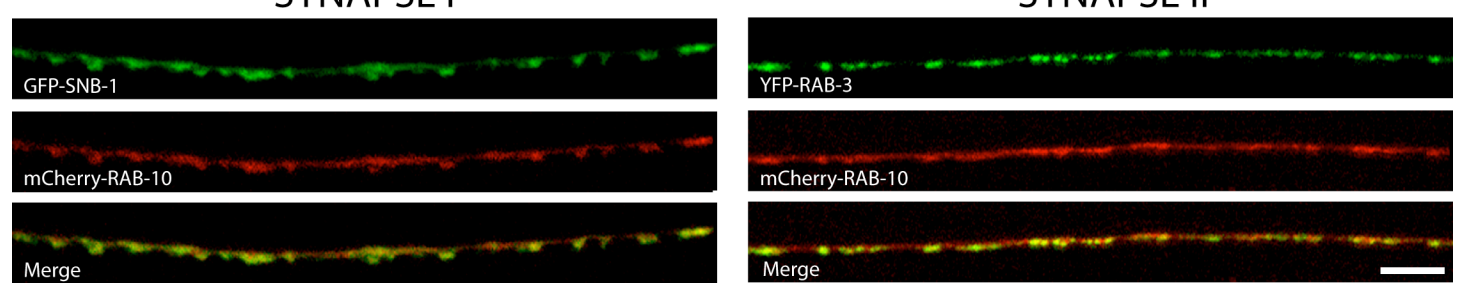

Figure VIII.15. Fine mapping of the sub-cellular localization of RAB-10 in neurons. A detailed analysis of the sub-cellular localization of RAB-10 showed that it was localized to discrete puncta in the neuronal cell soma and at the synapses of DNC. Neuronal cell somas from the VNC were chosen for imaging and each picture depicts two neuronal cell bodies. In the cell bodies, RAB-10 showed partial colocalization with Golgi and endosomal markers, but no full colocalization with any of the markers tested. At the synapses, RAB-10 showed a complete localization with SNB-1 and RAB-3. However, the RAB-10 staining was less concentrated than the SV markers. Note: GFP-RAB-10 and tagRFP-SYX-6 are false colored to enable simpler viewing. Scale bar in somas is $4 \mu \mathrm{m}$ and DNC is 5 $\mu \mathrm{m}$.

be localizing to DCVs, since there are fewer DCVs than SVs at C. elegans synapses (Fig. VIII.8).

After having analyzed the localization of RAB-10, the localization of the RAB-5 (Q78L), TBC-2, RABN-5, TBC-4 and EHBP-1 was elucidated. RAB-5 (Q78L) localized on domains adjacent to RAB-10. TBC-4 had a full overlap with RAB-10, which is in accordance with the previous data that it is a potential GAP for RAB-10. Additionally, TBC-4 showed a full colocalization with RABN-5. This is also in accordance with the previous $\mathrm{Y} 2 \mathrm{H}$ binding data. EHBP-1 had a rather diffuse and tubular staining, but it did show a partial overlap with RAB-10 (Fig. VIII.16). 

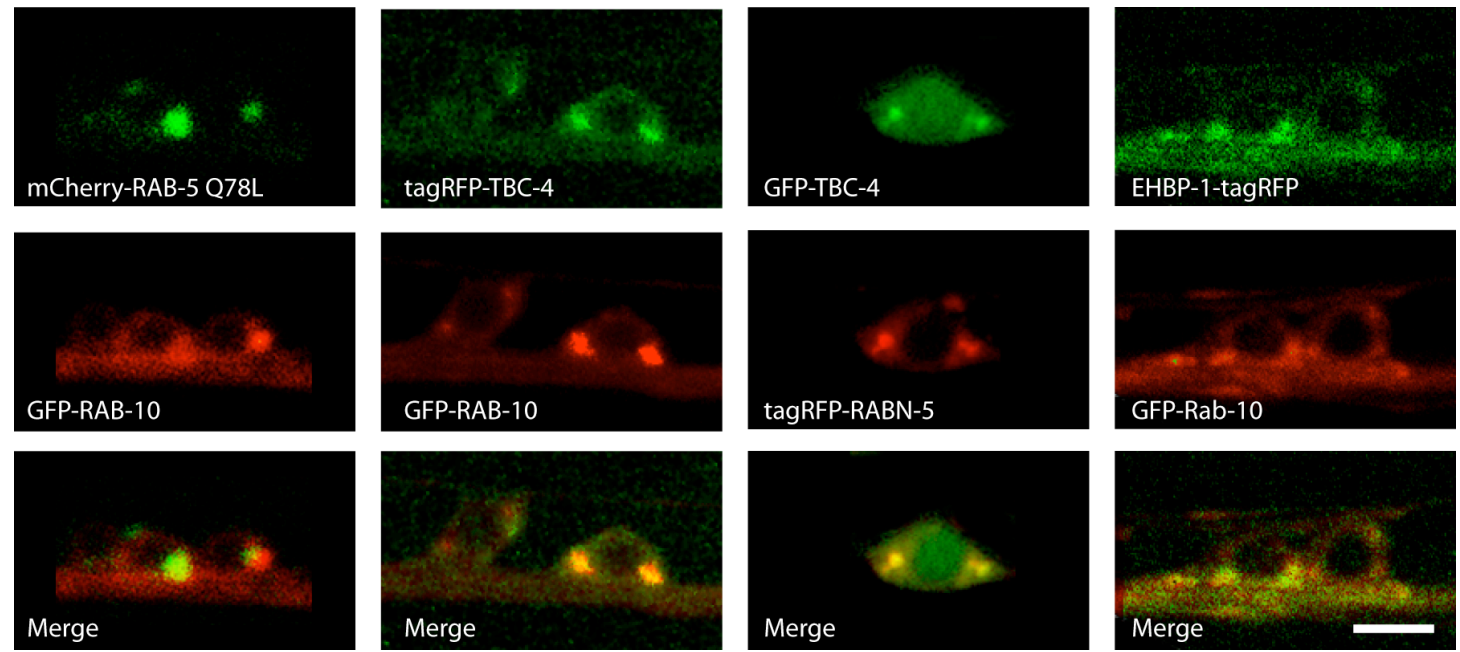

Figure VIII.16. Sub-cellular localization analysis of RAB-5 (Q78L), TBC-4, RABN-5 and EHBP1. All images were taken of neurons from the VNC. RAB-5 (Q78L) appeared to localize adjacent to RAB-10. TBC-4 displayed full colocalization with RAB-10. A significant amount of TBC-4 was also cyotosolic. RABN-5 and RABN-5 also showed a full overlap in localization. EHBP-1 and RAB-10 displayed a partial overlap. EHBP-1 staining was diffuse and tubular in the neuronal cell bodies. Note: GFP-RAB-10, mCherry-RAB-5 (Q78L), tagRFP-TBC-4 and EHBP-1-tagRFP were false colored to allow simpler viewing. Scale bar in somas is $4 \mu \mathrm{m}$.

This concludes the experiments conducted during the course of this study. The results point towards the existence of a RAB cascade necessary for DCV secretion whereby RAB-5 recruits TBC-4 via RABN-5. After recruitment, TBC-4 would then exclude RAB-10 from nearby membranes allowing for mDCVs to form. Details of this cascade can be found in the IX. Chapter 2: Discussion section. Further experiments are ongoing to elucidate the mechanism of this process. 


\section{DISCUSSION}

\subsection{RAB-5 and RAB-10 are novel regulators of DCV secretion}

RAB GTPases are master regulators of membrane trafficking. To understand the role of RABs in regulating DCV release, we took advantage of a novel in vivo fluorescence based assay to study DCVs in C. elegans (Sieburth et al., 2007). In wild type animals NLP-21-YFP is secreted from the motor neurons into the ECM. Consequently, macrophage-like cells called coelomocytes endocytose the YFP. Secretion of YFP can be indirectly quantified by means of fluorescence intensities in the coelomocytes. Although, this is an indirect measure of secretion, previously identified molecules involved in DCV release in mammalian cells have also been shown to be defective in NLP-21-YFP secretion: CAPS (C.e UNC-31), Munc-13 (C.e UNC-13), tomosyn (C.e TOM-1) (Sieburth et al., 2007; Gracheva et al., 2007). Furthermore, this assay is amenable for conducting larger scale analyses of multiple mutants. Therefore we tested all available rab mutants through this assay to study the role of RABs in regulating DCV function.

Analysis of all $r a b$ GTPase mutants showed that constitutively active $r a b-5$ Q78L and rab-10 mutants had severe defects in NLP-21-YFP secretion. These results were further confirmed by testing with a second DCV marker, INS-22-YFP, suggesting these mutants have a general defect in DCV secretion. To ensure that the observed defect was cell-autonomous in neurons we developed a neuron-specific RNAi technique. In accordance with the analysis of the mutants, neuron-specific knockdown of rab-5 and rab-10 also suggested that they are essential for DCV release. Since the RNAi results of rab-5 phenocopied $r a b-5 Q 78 L$, for simplicity the rab-5 $Q 78 L$ mutants will be referred to as $r a b-5$ mutants for the remainder of the discussion. 
Unlike the unc-31 (CAPS) mutants, which also have a defect in DCV secretion, the $r a b-5$ and $r a b-10$ mutants displayed no buildup of unsecreted YFP at the synapses. Rather, the fluorescence intensities at the DNCs of these mutants were like wild type. An explanation for this stems from the finding that de novo DCV biogenesis is genetically regulated by a DCV transmembrane protein, IA2/ICA512, through a feedback mechanism (Trajkovski et al., 2008). Upon fusion, the cytoplasmic tail of IA2/ICA512 is cleaved and translocates to the nucleus to positively regulate the expression of DCV proteins (Trajkovski et al., 2008). In the absence (or severe decrease) of fusion, like in $r a b-5$ and $r a b-10$ mutants, such a feedback loop could be inhibited. This would consequently arrest the production of new DCVs, preventing an accumulation at synapses. C. elegans has a single orthologue of IA2/ICA512 called IDA-1 and interestingly, it has been recently shown to localize to DCVs in neurons and to be involved in the regulation of DCV genes (Cai et al., 2009).

In order to identify novel molecules that may function together with RAB-5 and RAB-10 we analyzed the TBC-domain containing family of proteins, which act as regulators of RAB GTPase function. Screening of all available neuronally expressed $t b c$ mutants showed that $t b c-2$ and $t b c-4$ are also involved in the DCV secretion pathway. To distinguish whether RAB-5, TBC-2, RAB-10 and TBC-4 were specific regulators of DCV release and not SV release, we analyzed the SVs in these mutants. Examination of fluorescent synaptic markers (GFP-SNB-1 and YFP-RAB-3) showed that $r a b-5, t b c-2, r a b-10$ and $t b c-4$ mutants had no changes in the amount of $\mathrm{SVs}$, the size of synapses or the number of synapses. Ultrastructural analysis of these mutants further revealed that the numbers of SVs were normal and that the distribution of SVs from the active zone were unchanged. Measurement of EPSCs revealed that evoked SV release was also unaffected in $r a b-5, t b c-2, r a b-10$ and $t b c-4$ mutants. This suggested that RAB-5, TBC-2, RAB-10 and TBC-4 were indeed specific regulators of DCV exocytosis and not SV release.

Considering the results that were obtained, we had hypothesized that TBC-2 and TBC-4 may function as GAPs for RAB-5 and RAB-10. Interestingly, in line with this prediction, recent in vitro studies showed that TBC-2 displayed GAP activity specifically for RAB-5 (Chotard et al., 2010). This intuitively suggested to us that TBC-4 may be the GAP for RAB-10. Since, attempts to recombinantly express RAB10 were unsuccessful, we used a $\mathrm{Y} 2 \mathrm{H}$ approach to determine the substrate specificity 
of TBC-4. Interestingly, TBC-4 bound to RAB-10 at its TBC domain suggesting that RAB-10 is indeed the GAP for RAB-10. Interestingly, TBC-4 also interacted with RAB-8 and RAB-11.1 at sites away from its TBC domain. Since depletion of rab-8 and $r a b-11.1$ showed no DCV secretion defect, the meaning of these interactions is unclear. It could be that TBC-4 is recruited by RAB- 8 and $\mathrm{RAB}-11.1$ to regulate other trafficking pathways.

A major question in this study was to determine how RAB-5 and RAB-10 cooperate together in regulating DCV secretion? In order to answer this question and identify a link between RAB-5 and RAB-10, we screened known RAB-5 effectors for interaction with RAB-10 and its effectors. RABN-5, a RAB-5 effector, was found to bind to $\mathrm{TBC}-4$ at $\mathrm{CC}$ domains distinct from the $\mathrm{TBC}$ domain. Interestingly knockdown of RABN-5 phenocopied the DCV defect of rab-5 and rab-10 mutants confirming its involvement in this pathway. Mammalian rabaptin-5 has been shown to form a complex with Rab5 and Rabex-5, and acts as stimulator of the GEF activity of Rabex-5 on Rab5. This in turn leads to a polymerization of Rab5 in the early endocytic pathway (Stenmark et al., 1995; Lippe et al., 2001). From the phenotype of rabn-5 mutants it was not clear if the effect of RAB-5 and RABN-5 on DCV release was an indirect consequence of a perturbed endocytic pathway. To distinguish these two functions, we tested another molecule necessary for homotypic fusion during endocytosis, EEA-1 (Simonsen et al., 1998). Interestingly, knockdown of EEA-1 did not result in decreased DCV secretion. This suggests that RABN-5 has a specific secondary function in regulating $\mathrm{DCV}$ exocytosis by recruiting TBC-4 for the local inactivation of RAB-10.

\subsection{A RAB-5 / RAB-10 exclusion cascade}

It has been shown that, inside the cell, multiple Rab GTPases can work together in a cascade to provide directionality during compartment maturation. Two distinct types of cascades have been identified: Rab conversions and Rab exclusions. In a Rab conversion, a specific Rab recruits the GEF of a secondary Rab allowing for a compartment to sequentially acquire a new Rab label. The best example of such a cascade is seen between Rab5 and Rab7. Early endosome localized Rab5 molecules recruit the HOPS complex, which possesses GEF activity for Rab7, leading to maturation of Rab5 positive compartments to a Rab7 positive compartments (Rink et al., 2005). In contrast, during Rab exclusion, a specific Rab recruits the GAP of 
secondary Rab allowing for inactivation of the secondary Rab during compartmental maturation. This is seen between the yeast Rabs, Ypt1 and Ypt31. Ypt31 recruits Gyp1, which is a GAP for Ypt1. This allows for exclusion of Ypt1 from Ypt31 positive membranes during transport through the Golgi (Rivera-Molina and Novick, 2009).

The results of this study provide evidence for the existence of a potential Rab exclusion cascade between RAB-5 and RAB-10 to regulate DCV function. In this cascade, RAB-5 would indirectly recruit TBC-4 through its interaction with RABN-5. The presence of TBC-4 on RAB-5 positive membranes would then locally inactivate RAB-10 excluding it from a RAB-5 domain. Considering that the binding of RAB-10 to EHBP-1 is also essential for DCV release, it is likely that the inactivated RAB-10 would be recruited to an EHBP-1 positive compartment (Fig. IX.1). The finding that, EHBP-1, unlike classical Rab-Effectors, is autonomously targeted to membranes, would support this (Shi et al., 2010). An interesting result was that RAB-10 did not interact with the full length of EHBP-1, but only to the isolated CC domain. An explanation could be that the $\mathrm{CC}$ binding site for RAB-10 is kept inaccessible until the end of the RAB-5 / RAB-10 cascade. Additional studies need to be done to determine how the full length EHBP-1 interaction with RAB-10 is modulated. RAB8 also interacted with the CC domain of EHBP-1, but the function of this interaction is unclear since rab-8 mutants displayed no defect in DCV secretion. It is possible that RAB-8 still contributes a mild role, which can be compensated by RAB-10, but not vice versa. The fact that both genes are synthetically lethal and that $r a b-8$ mutants generally display milder phenotypes would support this (Chapter 1).

The necessity for DCVs to mature through a RAB-5 / RAB-10 cascade may explain why disruption of any one component leads to a defect in DCV secretion. Multiple studies have suggested that constitutively active Rabs display phenotypes, which are distinctly different from constitutively inactive Rabs (Stenmark et al., 1994; Ullrich et al., 1996; Junutula et al., 2004). This was not the case in our study, as we observed similar phenotypes between dominant mutants of RABs and depletion (by deletion or RNAi) of the same RABs. This suggests that certain complex processes such as DCV maturation and secretion require many molecules to ensure fidelity. In such a process, there is a demand for fully functional Rab cycles, which would be equally disrupted by a constitutively inactive or active Rab. Instances where a constitutively inactive or active Rab displays similar phenotypes have been previously 
A

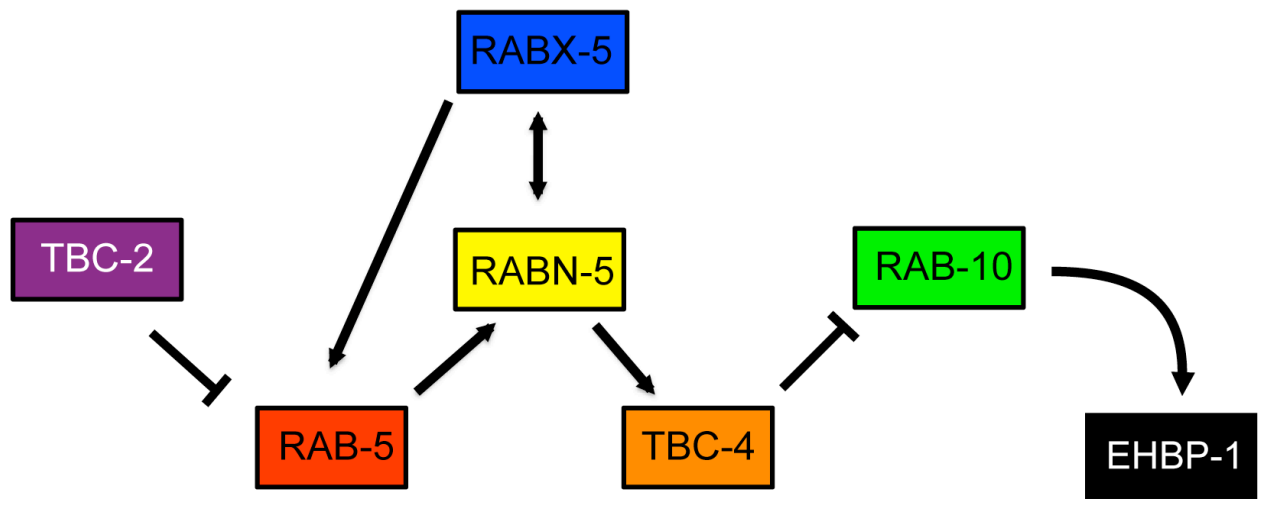

B

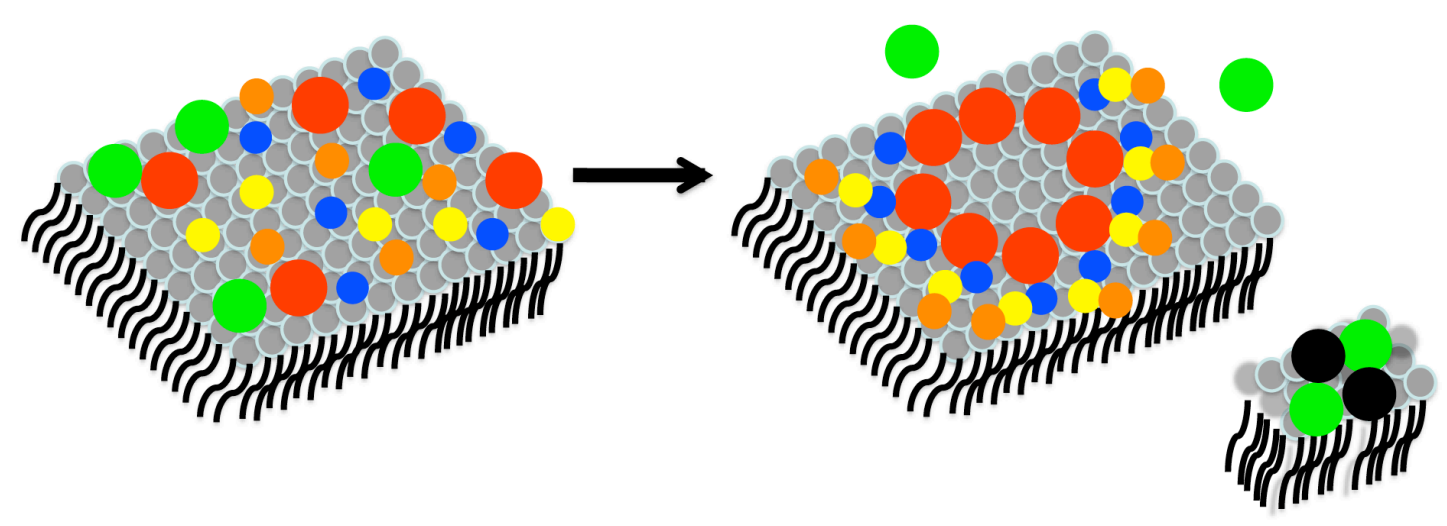

Figure IX.1. Model of a RAB-5 / RAB-10 exclusion cascade. A. The results of this study suggest the existence of a RAB exclusion cascade, which regulates DCV function. In this cascade RAB-5 recruitment to a compartment simultaneously leads to the recruitment of RABN-5. The presence of RABN-5 stimulates the GEF activity of RABX-5 towards RAB-5 (Lippe et al., 2001) and at the same time locally inactivates RAB-10. The inactivated RAB-10 would then re-localize to a neighboring a neighboring microdomain containing EHBP-1. All molecules would need to work in concert for the formation of fusion competent DCVs. B. A schematic representation of how all molecules would work in concert. TBC-2 is not depicted as it is a negative regulator of this pathway and it is likely that it is necessary to switch off this entire process.

reported. Expression of constitutively active or inactive mutants of Rab27 in neurons displayed defects in recycling of SVs (Pavlos et al., 2010). Expression of constitutively active or inactive Rab6 in HeLa cells displayed decreased hemaggluttinin transport to the cell surface (Martinez et al., 1994). 


\subsection{Localization of the RAB-5/RAB-10 cascade: cell body or synapse?}

Considering that RAB-5 and RAB-10 localize to discrete compartments in neuronal cell somas as well as to synapses (Brown et al., 2009; this study), a major question, which remains to be answered is, where does the RAB-5 / RAB-10 exclusion cascade occur? Given the current set of data, it is difficult to discriminate between the neuronal cell soma and the synapse. If it were to take place in the cell soma, then this cascade would likely occur in synchrony with the maturation process of iDCVs to mDCVs. In this scenario, disruption of the RAB-5 / RAB-10 cascade would lead to missorting of essential factors necessary for mDCV formation. Three such factors are: Syntaxin 6, VAMP4 and Synaptotagmin-IV. All three of these proteins are found on iDCVs and not on mDCVs in neuroendocrine PC12 cells (Tooze et al., 2001). Syntaxin 6 has been shown to be necessary for the homotypic fusion events preceding $\mathrm{mDCV}$ formation and is subsequently sorted to the endolysosomal system (Wendler et al., 2001). Synaptotagmin-IV has been shown to bind to syntaxin 6 to regulate homotypic fusion events (Ahras et al., 2006). Interestingly, recent studies in neurons has shown that certain amounts of synaptotagmin IV are also found on mDCVs and that it acts as an inhibitor of DCV release (Dean et al., 2009; Zhang et al., 2009). These results suggest the modulation of synaptotagmin-IV levels have consequences on DCV secretion. It will be interesting to determine the localization of syntaxin 6 and synaptotagmin IV in $r a b-5$ and $r a b-10$ mutants. It is possible that these factors are missorted onto mDCVs. It will also be interesting to conduct a proteomic analysis of DCV content in $r a b-5$ and $r a b-10$ mutants to identify additional factors that may be missorted in these mutants.

In the second scenario, it is possible that the RAB-5 / RAB-10 cascade occurs at the synapse, perhaps on DCVs. A recent finding that mammalian Rab5 and Rab10 are found on purified DCVs from rat spinal cord neurons, would support this (Zhao et al., 2011). It is unlikely that these RABs are involved in DCV transport since the DCVs are able to reach the synapse in these rab-5 and rab-10 mutants. One possibility is that, once at the synapse, DCVs must form specific microdomains prior to fusion. A functional RAB-5/ RAB-10 cascade would lead to the formation of specific RAB-5 and RAB-10 microdomains. In the absence of these microdomains the DCVs may be rendered fusion incompetent. This raises the question: what is the purpose of these microdomains. A simple suggestion would be that they are necessary for efficient tethering of DCVs, prior to fusion. Previous studies have implicated 
Rab10 in the insulin-triggered translocation of glucose transporter 4 (GLUT4) vesicles (GSVs) to the plasma membrane in adipocytes. Depletion of Rab10 leads to a drastic decrease in exocytosis of GSVs suggesting a role of this Rab in fusion (Sano et al., 2007; Sano et al., 2008). An appealing idea would be that the role of Rab10 in GSV fusion is analogous to its role in DCV fusion in C. elegans. Interestingly, in line with this, additional studies have implicated EHBP-1 and TBC1D4 (a mammalian Rab10 GAP) in GLUT4 translocation (Guilherme et al., 2004; Minea et al., 2005). To date, a role of Rab-5 has not been established, nor a formal RAB-5 / RAB-10 exclusion cascade described in GSV exocytosis. Additional work needs to be conducted to elucidate the potential role of these molecules to verify if the GLUT4 translocation pathway is similar to that of DCV release.

Alternatively, the requirement of the RAB-5 / RAB-10 exclusion cascade at the synapse may involve a role of the actin cytoskeleton. Presynaptic axon terminals have been shown to contain large amounts of filamentous actin (Fifkova and Dilay, 1982; Landis et al., 1988). SVs of the reserve pool were shown to be linked to the actin cytoskeleton through proteins called synapsins (Hilfiker et al., 1999). Synapsins were shown to act as negative regulators of high frequency induced SV exocytosis (Pieribone et al., 1995; Li et al., 1995). Interestingly, it has also been shown recently that synapsins act as negative regulators of DCV release in chromaffin cells (Villanueva et al., 2006). Parallel studies in chromaffin cells have also revealed that actin remodeling contributes significantly to the release of cargoes from DCVs (Felmy, 2007) and that the interaction between an actin motor protein, myosin-Va, and syntaxin-1 plays a role in DCV release (Watanabe et al., 2005). These studies suggest that DCVs are anchored on actin filaments and that exocytosis of DCVs is modulated by actin rearrangements at the synapse. Interestingly EHBP-1 contains a calponin homology $(\mathrm{CH})$ domain. Since, $\mathrm{CH}$ domains bind to actin filaments (Korenbaum and Rivero, 2002), it is possible that the interaction of RAB-10 with EHBP-1 is necessary for a remodeling of the synaptic actin cytoskeleton. Such remodeling could be necessary to enable DCV mobility prior to fusion. In this case, the disruption of the RAB-5 / RAB-10 exclusion cascade would trap the DCVs at the synapses preventing them from fusing. Additional studies on the effect of EHBP-1 on synaptic actin dynamics would help to elucidate its role in DCV release. 
In summary, we have identified through a systematic analysis of all RABs that RAB-5 and RAB-10 are novel regulators of DCV secretion in C. elegans. We have proposed that these molecules work together through a RAB exclusion cascade where active RAB-5 inactivates RAB-10 through recruiting TBC-4. This cascade is thought to occur in the neuronal cell soma or at the synapse. Furthermore, it was shown that disruption of the cascade perturbs DCV release. It is likely that this defect occurs either due to missorting of essential factors for fusion, failure to form the appropriate Rab microdomains necessary for tethering, or due to incorrect actin remodeling necessary for fusion. 


\section{Materials ANd Methods}

\subsection{Molecular Cloning}

Molecular cloning was conducted using classical recombinant molecular biology techniques (Sambrook and Russel, 2001). All bacterial strains and constructs used for cloning are listed in the Appendix. Each construct is described with the primers used for PCR and restriction sites used for cloning. DNA sequences of all primers (Invitrogen) can also be found in the Appendix. All genes of interest were PCR amplified out of a $C$. elegans cDNA library (Proquest). All genomic loci of interest were PCR amplified out of purified C. elegans genomic DNA (provided by Christina Pelzelt). PfuUltra II Polymerase (Stratagene) was used for all cloning PCR reactions. For genotyping PCR reactions Taq Polymerase (Invitrogen) was used. A standard $25 \mu \mathrm{l}$ PCR mix contained $1 \mu \mathrm{l}$ of each primer $[10 \mu \mathrm{M}], 2.5 \mu 1$ 10x PCR buffer (Stratagene/Invitrogen), $0.5 \mu \mathrm{dNTP}$ 's [10mM], 0.5 $\mu 1$ of Polymerase, 20-200 ng DNA template and the remaining volume was filled with double distilled $\mathrm{H}_{2} \mathrm{O}\left(\mathrm{ddH}_{2} \mathrm{O}\right)$.

Restriction enzymes, T4 DNA ligase and respective buffers were purchased from Fermentas and New England Biolabs. Restriction digest was typically conducted in a reaction volume of $20 \mu \mathrm{l}$, with $2 \mu \mathrm{l}$ of $10 \mathrm{x}$ digestion buffer, $0.5 \mu \mathrm{l}$ of each restriction enzyme, $1 \mu \mathrm{g}$ of DNA, and remaining volume filled with $\mathrm{ddH}_{2} \mathrm{O}$. Reactions were carried out for 2-3 hours at $37{ }^{\circ} \mathrm{C}$. Ligation reactions were conducted in a reaction volume of $20 \mu \mathrm{l}$, with $2 \mu \mathrm{l}$ of 10x ligation buffer, $1 \mu \mathrm{l}$ of ligase, $25 \mathrm{ng}$ DNA (total mass of vector and insert), and remaining volume filled with $\mathrm{ddH}_{2} \mathrm{O}$. Ligation reactions were carried out overnight at $15{ }^{\circ} \mathrm{C}$. A subset of constructs was cloned using the Invitrogen Gateway cloning system. LR reaction mixes consisted of a total volume of $20 \mu \mathrm{l}$ with $100 \mathrm{ng}$ of the entry clone, $100 \mathrm{ng}$ of destination vector, 2 $\mu \mathrm{l}$ of LR clonase, $8 \mu \mathrm{l}$ of TE buffer (Appendix) and the remaining volume filled with 
$\mathrm{ddH}_{2} \mathrm{O}$. LR reaction mix was incubated at $25^{\circ} \mathrm{C}$ for 1 hour. After reaction, $1 \mu \mathrm{l}$ of proteinase $\mathrm{K}$ was added and incubated at $37^{\circ} \mathrm{C}$ for 10 minutes to terminate the $\mathrm{LR}$ reaction. All PCR reactions and digestion products were analyzed on 1\% agarose gels (Invitrogen), run on 1x TBE buffer (Appendix).

All ligation mixes were transformed into $100 \mu \mathrm{l}$ chemi-competent DH5 $\alpha$ cells (Invitrogen) through the heat-shock transformation method. After mixing of ligation products with DH5 $\alpha$ cells, the mixture was allowed to stand for 25 minutes on ice. Then mixture was heat shocked for 2 minutes at $42^{\circ} \mathrm{C}$ and placed back on ice for 2 minutes. For recovery, $900 \mu \mathrm{l}$ of LB medium (Appendix) was added and then placed on a shaker at $37^{\circ} \mathrm{C}$ for 45 minutes. After recovery, cells were pelleted, resuspended and plated on to ampicillin or kanamycin containing LB-agar plates (Appendix). Following transformation, colonies were picked and inoculated with desired volume of LB media. Plasmid DNA was isolated using miniprep kits (Invitrogen) or midiprep kits (JetStar). For transformation of LR reaction products, DB3.1 cells were used.

\subsection{C. elegans genetics}

\subsubsection{Strains}

All strains were cultured at $20^{\circ} \mathrm{C}$ on OP50 E.coli-seeded Nematode Growth Medium (NGM) plates as previously (Brenner, 1974). All mutant C. elegans strains used in this study were ordered from the C. elegans Gene Knockout Consortium (CGC-Vancouver, Canada and Minnesota/Oklahoma, USA) and/or the National Bioresource Project for the Nematode C. elegans (Tokyo, Japan). Strains used in this study are listed in the Appendix.

\subsubsection{Crosses}

Males for crosses were induced via heatshock. 6 large NGM plates $(100 \mathrm{~mm})$, each containing $20 \mathrm{~L} 4 \mathrm{~N} 2$ worms was prepared. Plates were kept at $30^{\circ} \mathrm{C}$ for heatshock. 2 plates were removed at 5 hours, 2 plates were removed at 5.5 hours, and 2 plates were removed at 6 hours and kept at $20^{\circ} \mathrm{C}$. The F1 progeny were screened for males. Usually, $<5 \%$ of the population is positive. Males were then isolated and used to cross an allele of interest into a desired genetic background. After crossing, 
homozygous F2 progeny were selected through phenotypic selection or by worm PCR.

For worm PCR, animals needed to be first lysed. For worm lysis, few animals from a strain of interest were suspended in lysis buffer containing proteinase $\mathrm{K}$ (Appendix). Worms were first placed at $-80^{\circ} \mathrm{C}$ for $20 \mathrm{~min}$ to allow ice crystals to initially rupture tissue. Subsequently worms were transferred to $65^{\circ} \mathrm{C}$ for 1 hour to allow proteinase $\mathrm{K}$ to digest the worm extract. After lysis, proteinase $\mathrm{K}$ was inactivated with a $95^{\circ} \mathrm{C}$ step for $20 \mathrm{~min} .2 \mu \mathrm{l}$ of lysate was used as template for the worm PCR reaction. Note that worm PCR is carried for the detection of deletion mutant alleles. Primers are designed to bind outside a deletion locus and also inside the locus to confirm homozygosity. Lists of all deletion alleles that were used in this study along with the sizes of the deletions and the primers used to screen for the deletion can be found in the Appendix. The DNA sequences of the screening primers can also be found in the Appendix.

\subsubsection{Generation of transgenic lines}

Transgenic lines carrying extrachromosomal arrays were generated through microinjection as previously described (Mello and Fire, 1995). Injection mixes containing genes of interest, co-injection marker and pBluescript combined in a final concentration of $100 \mathrm{ng} / \mu \mathrm{l}$ were used for injections. All the injection mixes that were used describing the genes of interest, co-injection markers and respective concentrations used can be found in the Appendix. Usually two independent transgenic lines per injection mix were generated. The transgenic lines generated in this study are also listed in the Appendix, after the mutant strains.

A number of integrated transgenic lines were obtained from the CGC and from other C. elegans labs: nuIs 152[punc-129::gfp-snb-1], nuIs168[punc-129::venusrab-3], nuIs183[punc-129::nlp-21-venus] and nuis195[punc-129::ins-22-venus] were provided by Joshua Kaplan, (Massachusetts General Hospital, Boston, MA). ZxIs5[punc-17::chop-2(H134R)-yfp;lin-15(+)] $X \quad$ and $\quad$ zxIs6[punc-17::chop2(H134R)-yfp;lin-15(+)] IV were provided by Alexander Gottschalk (Johann Wolfgang Goethe-Universität, Frankfurt, Germany). BIs34[prme-8::rme-8-gfp] was provided by Barth Grant (Rutgers University, New Jersey, USA). 


\subsection{C. elegans behavioral assays}

\subsubsection{Movement analysis}

To assay worm movement, the number of body bends per minute was assayed. Individual animals were transferred from seeded NGM plates to non-seeded NGM plates. The number of sinusoidal body bends per minute was visually measured. Each animal was tested for three minutes and at least 10 animals were assayed per strain.

\subsubsection{Defecation motor program analysis}

For assaying defecation, two parameters were measured: Success of expulsion events and the cycle length between subsequent posterior body contractions (Thomas, 1990). A minimum of 10 animals per strain were analyzed and each animal was recorded for 5-10 minutes.

\subsubsection{Egg-laying analysis}

The egg-laying assay was performed as previously described (Patel et al., 2006.) 20 Young adult worms were transferred to fresh plates and after $30 \mathrm{~min}$, the developmental stage of each newly laid egg was assessed into three distinct stages: one-to-eight-cell stage, nine-cell-to-comma stage and postcomma stage.

\subsubsection{Pharmacological assays}

Pharmacological assays for synaptic transmission were conducted with aldicarb as described previously (Mahoney et al. 2006b). Seeded NGM plates containing $2 \mathrm{mM}$ aldicarb were prepared (Riedel de Haen). 30 animals per strain were transferred onto aldicarb plates and assessed after 90 minutes of exposure. The percentage of animals paralyzed was quantified. Paralysis was determined by failure to move after prodding with silver wire. Levamisole sensitivity assays were conducted as previously described (Lewis et al., 1980). NGM plates containing $1 \mathrm{mM}$ levamisole were prepared (Sigma Aldrich). 30 animals per strain were transferred onto levamisole plates and observed after 80 minutes of exposure. The percentage of animals paralyzed was quantified. 


\subsection{DiI staining}

A stock solution of $2 \mathrm{mg} / \mathrm{ml} \mathrm{1,1'-dioctadecyl-} \mathrm{3,} \mathrm{3,} \mathrm{3',} \mathrm{3'-}$ tetramethylindocarbocyanine perchlorate (DiI) (Molecular Probes) in N,Ndimethylformamide was stored at $-20^{\circ}$. For the working solution, the stock was diluted 1:200 in M9 buffer (Appendix). Strains were then soaked in the DiI working solution for 2-3 hours at room temperature. After staining, worms were washed 2-3x with M9 buffer and spotted onto NGM plates for destaining. 1 hour later worms were mounted onto $2 \%$ agarose pads for imaging (Bacaj et al., 2008).

\subsection{Chemosensation assay}

This assay was modified from a previously described protocol (Wicks, 2000). A solution of $150 \mathrm{mM} \mathrm{CuSO}_{4}$ with trace amounts of bromophenol blue was prepared. Bromophenol blue was used to color the solution to allow visibility under the light microscope. A line of copper sulfate solution was drawn across the midline of an unseeded NGM plate. The strain to be assayed was washed twice with S-Basal Buffer to remove bacteria and spotted to one side of the assay plate. $2 \mu \mathrm{l}$ of an attractant, isoamyl alcohol (diliuted 1:10 in ethanol) was placed on the opposite side of the plate across the line of copper sulfate. Wild type worms approach the attractant, but do not cross the line of copper sulfate. The ability of worms to be attracted to isoamyl alcohol and avoid copper was qualitatively assessed.

\subsection{RNAi by feeding}

Genes of interest were knocked down by feeding RNAi as previously described (Kamath et al., 2001). All genes for RNAi experiments were cloned into L4440, a vector with two opposing T7 polymerase binding sites flanking the multiple cloning site. The vectors were then transformed into E.coli HT115 (DE3) strain. The overnight culture grown in LB media, was seeded onto NGM plates containing 100 $\mu \mathrm{g} / \mathrm{ml}$ ampicillin and $1 \mathrm{mM}$ isopropyl $\beta$-D-thiogalactopyranoside (IPTG). 10 L4 animals of strains of interest in an eri-1(mg366) background, were transferred onto plates and allowed to lay eggs for 12 hours. The animals were then transferred to a second round of plates and allowed to lay eggs for another 12 hours. Parents were then removed and the progeny were analyzed/scored for phenotypes. All the RNAi constructs that were cloned can be found in the Appendix. Table III.2 lists all the 
strains of interest that were crossed into the eri-1(mg366) background. For neuronspecific RNAi, the eri-1(mg366);rde-4(ne301); gz133 [punc-129::rde-4] strain was used.

\subsection{Confocal microscopy and image analysis}

Animals were immobilized with $50 \mathrm{mM}$ Na-azide and placed on 2\% agarose pads. All imaging was performed on an inverted confocal microscope (SP2, Leica). Images were taken using a 63x NA 1.32 / 40x NA 1.25 oil immersion objectives at 20 ${ }^{\circ} \mathrm{C}$. Stacks of regions of interest were taken and then compiled to generate maximum intensity projections using Leica software.

For expression pattern analysis segments of worms were separately imaged and a composite image of a full-length worm was generated using Photoshop. For sub-cellular localizations, images of neuronal cell somas from the VNC were taken. Images for quantification from DNC and coelomocytes was carried out as described previously (Sieburth et al., 2005; Sieburth et al., 2007). Maximum stack projections taken from the confocal software were thresholded and quantified using ImageJ software (National Institutes of Health) as described previously (Sumakovic et al., 2009). All data was normalized to wild type, where indicated.

For imaging of the DyI stained worms an inverted Olympus IX81 microscope combined with a PerkinElmer Ultraview Vox spinning disk setup was used. A 40x NA 0.75 air immersion lens was used for imaging. Stacks of regions of interest were taken and then compiled to generate maximum intensity projections using PerkinElmer software.

\subsection{TR-BSA endocytosis assay}

The endocytosis of the fluid-marker (TR-BSA), was monitored as described previously (Zhang et al., 2001). Strains of interest were crossed into the bIs34 strain to label early endosomes. $1 \mathrm{mg} / \mathrm{ml}$ of TR-BSA was injected into the pseudocoelom near the pharynx of adult animals. After specific time points $10 \mathrm{mins}, 30 \mathrm{mins}$, and 45 mins, animals were transferred onto chilled NGM plates to retard trafficking and prepared subsequently for imaging. At least 4 different animals were injected and imaged for each time point. 


\subsection{High-pressure freeze electron microscopy}

A $100 \mu \mathrm{m}$ deep aluminium platelet (Microscopy Services, Flintbek) was filled with E.coli OP 50 suspension. About 20 young adult worms were transferred into the chamber and immediately frozen using a BalTec HPM 10. Freeze substitution was carried out in a Leica AFS2. Incubations were at $-90{ }^{\circ} \mathrm{C}$ for $100 \mathrm{~h}$ in $0,1 \%$ tannic acid, $7 \mathrm{~h}$ in $2 \% \mathrm{OsO} 4$, and at $-20{ }^{\circ} \mathrm{C}$ for $16 \mathrm{~h}$ in $2 \% \mathrm{OsO} 4$, followed by embedding in EPON at RT (Rostaing et al., 2004) (all solutions w/v in dry acetone). $50 \mathrm{~nm}$ sections were mounted on copper slot grids and placed for $10 \mathrm{~min}$ on drops of $4 \%(\mathrm{w} / \mathrm{v})$ uranyl acetate in $75 \%$ methanol and then washed in destilled water. After air drying the grids were placed on lead citrate (Reynolds, 1963) for $2 \mathrm{~min}$ in a CO2-free chamber, and rinsed in destilled water. Micrographs were taken with a $1024 \times 1024$ CCD detector (Proscan CCD HSS 512/1024; Proscan Electronic Systems, Scheuring, Germany) in a Zeiss EM 902A, operated in the bright field mode.

\subsection{Electrophysiology}

Recordings from dissected C. elegans body wall muscle cells were conducted as described previously (Liewald et al., 2008). After dissection, cells were treated for $8 \mathrm{~s}$ with $0.5 \mathrm{mg} / \mathrm{ml}$ collagenase (Sigma-Aldrich, Germany) in modified Ascaris Ringer's (AR; $150 \mathrm{mM} \mathrm{NaCl}, 5 \mathrm{mM} \mathrm{KCl}, 5 \mathrm{mM} \mathrm{CaCl}, 1 \mathrm{mM} \mathrm{MgCl} 2,10 \mathrm{mM}$ glucose, $15 \mathrm{mM}$ HEPES (pH 7.35), $340 \mathrm{mOsm}$ ) and washed with AR. Cells were clamped to a holding potential of $-60 \mathrm{mV}$ using an EPC10 amplifier and analyzed by Pulse software (HEKA Electronics, Germany). The bath solution was AR, the pipette solution was $120 \mathrm{mM} \mathrm{KCl,} 20 \mathrm{mM} \mathrm{KOH}, 4 \mathrm{mM} \mathrm{MgCl}_{2}, 5 \mathrm{mM}$ TRIS-HCl (pH 7.2), $0.25 \mathrm{mM} \mathrm{CaCl}_{2}, 4 \mathrm{mM}$ ATP, $36 \mathrm{mM}$ sucrose, and $5 \mathrm{mM}$ EGTA (315 mOsm). Light activation was performed using a LED lamp (KSL-70, Rapp OptoElectronic, Germany) at a wavelength of $470 \mathrm{~nm}\left(8 \mathrm{~mW} / \mathrm{mm}^{2}\right)$, and controlled by the HEKA amplifier software.

\subsection{Yeast-2-hybrid}

The Matchmaker yeast two-hybrid assay was performed according to manufacturers protocol (Clontech). All dominant active and dominant negative Rabs were cloned into the bait vector pGBKT7, whereas $t b c-4, t b c-4$ (377-825), rabn-5 and $t b c-4$ R155A were cloned into the prey vector pGADT7 (Clontech). All yeast-2hybrid vectors are listed in table III.1. The plasmid combinations of interest were 
transformed into the yeast strain AH109 (Clontech) and tested for interaction by spotting on selective plates lacking histidine. Composition of YAPD, transformation and spotting plates can be found in the Appendix.

\subsection{Protein expression and purification}

All $r a b$ genes were cloned into the pGST (Glutathione-S-transferse) parallel II vector (Sheffield et al., 1999). Vectors were transformed into the E. coli BL21 star (DE3) strain (Invitrogen). Protein expression was induced with $0.1-1 \mathrm{mM}$ IPTG at an OD range of 0.4-0.6. Expression was stopped after 4 hours. Cells were pelleted and resuspended in cleavage buffer (Appendix). The solution was sonicated on ice and centrifuged at $11500 \mathrm{~g}$ (SS-34 rotor) for 30 minutes to remove cellular debris. The supernatant was incubated with GST-Sepharose beads (GE healthcare) for one hour on room temperature. Then washed $3 \mathrm{x}$ with $3 \mathrm{~mL}$ PBS and eluted with GST elution buffer (Appendix). Purity of protein was then judged by boiling $10 \mu$ of protein sample in SDS and running samples through SDS-PAGE. SDS gels were prepared and run using a BioRad Mini-PROTEAN 3 cell electrophoresis system and 1x SDS running buffer (Appendix). $10 \%$ gels were used and run at $160 \mathrm{~V}$ for $60-90$ minutes. Gels were stained with Coomassie Brilliant Blue R250 and destained with Coomassie destaining buffer (Appendix). 


\section{REFERENCES}

Ahmed, S., Maruyama, I.N., Kozma, R., Lee, J., Brenner, S., and Lim, L. (1992). The Caenorhabditis elegans unc-13 gene product is a phospholipid-dependent highaffinity phorbol ester receptor. Biochem J 287 ( Pt 3), 995-999.

Ahras, M., Otto, G.P., and Tooze, S.A. (2006). Synaptotagmin IV is necessary for the maturation of secretory granules in PC12 cells. J Cell Biol 173, 241-251.

Alberts, B., Johnson, A., Lewis, J., Raff, M., Roberts, K., and Walter, P. (2008). Molecular Biology of the Cell, 5th Edition. Garland Science, 751.

Ali, B.R., Wasmeier, C., Lamoreux, L., Strom, M., and Seabra, M.C. (2004). Multiple regions contribute to membrane targeting of Rab GTPases. Journal of Cell Science $117,6401-6412$.

Altun, Z.F., and Hall, D.H. (2008). Handbook of C. elegans anatomy. WormAtlas, www.wormatlas.org/hermaphrodite/hermaphroditehomepage.htm.

Ann, K., Kowalchyk, J.A., Loyet, K.M., and Martin, T.F. (1997). Novel Ca2+binding protein (CAPS) related to UNC-31 required for Ca2+-activated exocytosis. J Biol Chem 272, 19637-19640.

Ashery, U., Varoqueaux, F., Voets, T., Betz, A., Thakur, P., Koch, H., Neher, E., Brose, N., and Rettig, J. (2000). Munc13-1 acts as a priming factor for large densecore vesicles in bovine chromaffin cells. The EMBO Journal. 19, 3586-3596.

Babbey, C.M., Ahktar, N., Wang, E., Chen, C.C.-H., Grant, B.D., and Dunn, K.W. (2006). Rab10 regulates membrane transport through early endosomes of polarized Madin-Darby canine kidney cells. Mol Biol Cell 17, 3156-3175.

Babbey, C.M., Bacallao, R.L., and Dunn, K.W. (2010). Rab10 associates with primary cilia and the exocyst complex in renal epithelial cells. Am J Physiol Renal Physiol 299, F495-506.

Bacaj, T., Lu, Y., and Shaham, S. (2008). The Conserved Proteins CHE-12 and DYF11 Are Required for Sensory Cilium Function in Caenorhabditis elegans. Genetics 178, 989-1002. 
Barbero, P., Bittova, L., and Pfeffer, S.R. (2002). Visualization of Rab9-mediated vesicle transport from endosomes to the trans-Golgi in living cells. J Cell Biol 156, 511-518.

Bargmann, C.I. (2006). Chemosensation in C. elegans. WormBook, 1-29.

Barlowe, C., Orci, L., Yeung, T., Hosobuchi, M., Hamamoto, S., Salama, N., Rexach, M.F., Ravazzola, M., Amherdt, M., and Schekman, R. (1994). COPII: a membrane coat formed by Sec proteins that drive vesicle budding from the endoplasmic reticulum. Cell 77, 895-907.

Barr, F., and Lambright, D.G. (2010). Rab GEFs and GAPs. Curr Opin Cell Biol 22, 461-470.

Boulin, T., Etchberger, J.F., and Hobert, O. (2006). Reporter gene fusions. WormBook, 1-23.

Branicky, R., and Hekimi, S. (2006). What keeps C. elegans regular: the genetics of defecation. Trends Genet 22, 571-579.

Brenner, S. (1974). The genetics of Caenorhabditis elegans. Genetics 77, 71-94.

Brose, N. (2008). For better or for worse: complexins regulate SNARE function and vesicle fusion. Traffic 9, 1403-1413.

Brose, N., Hofmann, K., Hata, Y., and Südhof, T.C. (1995). Mammalian homologues of Caenorhabditis elegans unc-13 gene define novel family of C2-domain proteins. J Biol Chem 270, 25273-25280.

Brown, H.M., Van Epps, H.A., Goncharov, A., Grant, B.D., and Jin, Y. (2009). The JIP3 scaffold protein UNC-16 regulates RAB-5 dependent membrane trafficking at C. elegans synapses. Dev Neurobiol 69, 174-190.

Burbach, J.P. (2010). Neuropeptides from concept to online database www.neuropeptides.nl. Eur J Pharmacol 626, 27-48.

Burguete, A.S., Fenn, T.D., Brunger, A.T., and Pfeffer, S.R. (2008). Rab and Arl GTPase family members cooperate in the localization of the golgin GCC185. Cell 132, 286-298.

Cai, T., Hirai, H., Fukushige, T., Yu, P., Zhang, G., Notkins, A.L., and Krause, M. (2009). Loss of the transcriptional repressor PAG-3/Gfi-1 results in enhanced neurosecretion that is dependent on the dense-core vesicle membrane protein IDA1/IA-2. PLoS Genet 5.

Cai, H., Reim, K., Varoqueaux, F., Tapechum, S., Hill, K., Sørensen, J.B., Brose, N., and Chow, R.H. (2008). Complexin II plays a positive role in Ca2+-triggered exocytosis by facilitating vesicle priming. Proc Natl Acad Sci USA 105, 1953819543. 
Cai, H., Reinisch, K., and Ferro-Novick, S. (2007). Coats, tethers, Rabs, and SNAREs work together to mediate the intracellular destination of a transport vesicle.

Developmental. Cell 12, 671-682.

Carroll, K.S., Hanna, J., Simon, I., Krise, J., Barbero, P., and Pfeffer, S.R. (2001). Role of Rab9 GTPase in facilitating receptor recruitment by TIP47. Science 292, 1373-1376.

Chapman, E.R. (2008). How does synaptotagmin trigger neurotransmitter release? Annu Rev Biochem 77, 615-641.

Chavrier, P., Gorvel, J.P., Stelzer, E., Simons, K., Gruenberg, J., and Zerial, M. (1991). Hypervariable C-terminal domain of rab proteins acts as a targeting signal. Nature 353, 769-772.

Chotard, L., Mishra, A.K., Sylvain, M.A., Tuck, S., Lambright, D.G., and Rocheleau, C.E. (2010). TBC-2 regulates RAB-5/RAB-7-mediated endosomal trafficking in Caenorhabditis elegans. Mol Biol Cell 21, 2285-2296.

Chow, R.H., Klingauf, J., and Neher, E. (1994). Time course of Ca2+ concentration triggering exocytosis in neuroendocrine cells. Proc Natl Acad Sci USA 91, 1276512769.

Chun, D.K., McEwen, J.M., Burbea, M., and Kaplan, J.M. (2008). UNC-108/Rab2 regulates postendocytic trafficking in Caenorhabditis elegans. Mol Biol Cell 19, 2682-2695.

Dal Santo, P., Logan, M.A., Chisholm, A.D., and Jorgensen, E.M. (1999). The inositol trisphosphate receptor regulates a 50 -second behavioral rhythm in $\mathrm{C}$. elegans. Cell 98, 757-767.

Dean, C., Liu, H., Dunning, F.M., Chang, P.Y., Jackson, M.B., and Chapman, E.R. (2009). Synaptotagmin-IV modulates synaptic function and long-term potentiation by regulating BDNF release. Nat Neurosci 12, 767-776.

Díaz, E., and Pfeffer, S.R. (1998). TIP47: a cargo selection device for mannose 6phosphate receptor trafficking. Cell 93, 433-443.

Donaldson, J., and Segev, N. (2009). Regulation and Coordinationof Intracellular Trafficking: An Overview. Madame Curie Bioscience, 1-13.

Duchaine, T.F., Wohlschlegel, J.A., Kennedy, S., Bei, Y., Conte, D., Pang, K., Brownell, D.R., Harding, S., Mitani, S., Ruvkun, G., et al. (2006). Functional proteomics reveals the biochemical niche of C. elegans DCR-1 in multiple smallRNA-mediated pathways. Cell 124, 343-354.

Eathiraj, S., Pan, X., Ritacco, C., and Lambright, D.G. (2005). Structural basis of family-wide Rab GTPase recognition by rabenosyn-5. Nature 436, 415-419.

Eaton, B.A., Haugwitz, M., Lau, D., and Moore, H.P. (2000). Biogenesis of regulated exocytotic carriers in neuroendocrine cells. J Neurosci 20, 7334-7344. 
Echard, A., Jollivet, F., Martinez, O., Lacapère, J.J., Rousselet, A., Janoueix-Lerosey, I., and Goud, B. (1998). Interaction of a Golgi-associated kinesin-like protein with Rab6. Science 279, 580-585.

Edwards, S.L., Charlie, N.K., Richmond, J.E., Hegermann, J., Eimer, S., and Miller, K.G. (2009). Impaired dense core vesicle maturation in Caenorhabditis elegans mutants lacking Rab2. J Cell Biol 186, 881-895.

Ehlers, M.D. (2000). Reinsertion or degradation of AMPA receptors determined by activity-dependent endocytic sorting. Neuron 28, 511-525.

Eimer, S., Gottschalk, A., Hengartner, M., Horvitz, H.R., Richmond, J., Schafer, W.R., and Bessereau, J.L. (2007). Regulation of nicotinic receptor trafficking by the transmembrane Golgi protein UNC-50. EMBO J 26, 4313-4323.

Elferink, L.A., Anzai, K., and Scheller, R.H. (1992). rab15, a novel low molecular weight GTP-binding protein specifically expressed in rat brain. J Biol Chem 267, 22693.

Espelt, M.V., Estevez, A.Y., Yin, X., and Strange, K. (2005). Oscillatory Ca2+ signaling in the isolated Caenorhabditis elegans intestine: role of the inositol-1,4,5trisphosphate receptor and phospholipases C beta and gamma. J Gen Physiol 126, 379-392.

Felmy, F. (2007). Modulation of cargo release from dense core granules by size and actin network. Traffic 8, 983-997.

Feng, Y., Press, B., and Wandinger-Ness, A. (1995). Rab7: an important regulator of late endocytic membrane traffic. J Cell Biol 131, 1435-1452.

Fifková, E., and Delay, R.J. (1982). Cytoplasmic actin in neuronal processes as a possible mediator of synaptic plasticity. J Cell Biol 95, 345-350.

Fischer von Mollard, G., Mignery, G.A., Baumert, M., Perin, M.S., Hanson, T.J., Burger, P.M., Jahn, R., and Südhof, T.C. (1990). rab3 is a small GTP-binding protein exclusively localized to synaptic vesicles. Proc Natl Acad Sci USA 87, 1988-1992.

Fukuda, M. (2011). TBC proteins: GAPs for mammalian small GTPase Rab? Biosci Rep 31, 159-168.

Fukuda, M., Kanno, E., Ishibashi, K., and Itoh, T. (2008). Large scale screening for novel rab effectors reveals unexpected broad Rab binding specificity. Molecular \& Cellular Proteomics 7, 1031-1042.

Fukuda, M., Kuroda, T.S., and Mikoshiba, K. (2002). Slac2-a/melanophilin, the missing link between Rab27 and myosin Va: implications of a tripartite protein complex for melanosome transport. J Biol Chem 277, 12432-12436.

Gally C, Eimer S, Richmond JE, Bessereau JL (2004) A trans-membrane protein required for acetylcholine receptor clustering in Caenorhabditis elegans. Nature 431: $578-582$ 
Gillingham, A.K., and Munro, S. (2007). The small G proteins of the Arf family and their regulators. Annu Rev Cell Dev Biol 23, 579-611.

Glick, B.S., and Nakano, A. (2009). Membrane traffic within the Golgi apparatus. Annu Rev Cell Dev Biol 25, 113-132.

Glodowski, D. R., Chen, C. C., Schaefer, H., Grant, B. D., and Rongo, C. (2007). RAB-10 regulates glutamate receptor recycling in a cholesterol-dependent endocytosis pathway. Mol. Biol. Cell 18, 4387-4396.

Goody, R.S., and Hofmann-Goody, W. (2002). Exchange factors, effectors, GAPs and motor proteins: common thermodynamic and kinetic principles for different functions. Eur Biophys J 31, 268-274.

Gracheva, E.O., Burdina, A.O., Touroutine, D., Berthelot-Grosjean, M., Parekh, H., and Richmond, J.E. (2007). Tomosyn Negatively Regulates CAPS-Dependent Peptide Release at Caenorhabditis elegans Synapses. Journal of Neuroscience 27, 1017610184.

Griffiths, G., Ericsson, M., Krijnse-Locker, J., Nilsson, T., Goud, B., Söling, H.D., Tang, B.L., Wong, S.H., and Hong, W. (1994). Localization of the Lys, Asp, Glu, Leu tetrapeptide receptor to the Golgi complex and the intermediate compartment in mammalian cells. J Cell Biol 127, 1557-1574.

Grishanin, R.N., Klenchin, V.A., Loyet, K.M., Kowalchyk, J.A., Ann, K., and Martin, T.F.J. (2002). Membrane association domains in Ca2+-dependent activator protein for secretion mediate plasma membrane and dense-core vesicle binding required for Ca2+-dependent exocytosis. J Biol Chem 277, 22025-22034.

Grosshans, B.L., Ortiz, D., and Novick, P. (2006). Rabs and their effectors: achieving specificity in membrane traffic. Proc Natl Acad Sci USA 103, 11821-11827.

Guilherme, A., Soriano, N.A., Furcinitti, P.S., and Czech, M.P. (2004). Role of EHD1 and EHBP1 in perinuclear sorting and insulin-regulated GLUT4 recycling in 3T3-L1 adipocytes. J Biol Chem 279, 40062-40075.

Guo, W., Roth, D., Walch-Solimena, C., and Novick, P. (1999). The exocyst is an effector for Sec $4 p$, targeting secretory vesicles to sites of exocytosis. The EMBO Journal 18, 1071-1080.

Haas, A.K., Fuchs, E., Kopajtich, R., and Barr, F.A. (2005). A GTPase-activating protein controls Rab5 function in endocytic trafficking. Nat Cell Biol 7, 887-893.

Hakoshima, T. (2003). Structural Basis of the Rho GTPase Signaling. Journal of Biochemistry 134, 327-331.

Hales, C.M., Vaerman, J.-P., and Goldenring, J.R. (2002). Rab11 family interacting protein 2 associates with Myosin $\mathrm{Vb}$ and regulates plasma membrane recycling. J Biol Chem 277, 50415-50421. 
Hammarlund, M., Watanabe, S., Schuske, K., and Jorgensen, E.M. (2008). CAPS and syntaxin dock dense core vesicles to the plasma membrane in neurons. J Cell Biol 180, 483-491.

Harrison, R.E., Brumell, J.H., Khandani, A., Bucci, C., Scott, C.C., Jiang, X., Finlay, B.B., and Grinstein, S. (2004). Salmonella impairs RILP recruitment to Rab7 during maturation of invasion vacuoles. Mol Biol Cell 15, 3146-3154.

Hatsuzawa, K. (2003). The R-SNARE Motif of Tomosyn Forms SNARE Core Complexes with Syntaxin 1 and SNAP-25 and Down-regulates Exocytosis. Journal of Biological Chemistry 278, 31159-31166.

Heiman, M.G., and Shaham, S. (2007). Ancestral roles of glia suggested by the nervous system of Caenorhabditis elegans. Neuron Glia Biol 3, 55-61.

Herman, M.A., and Horvitz, H.R. (1994). The Caenorhabditis elegans gene lin-44 controls the polarity of asymmetric cell divisions. Development 120, 1035-1047.

Hilfiker, S., Pieribone, V.A., Czernik, A.J., Kao, H.T., Augustine, G.J., and Greengard, P. (1999). Synapsins as regulators of neurotransmitter release. Philos Trans R Soc Lond B Biol Sci 354, 269-279.

Hilliard, M.A., Bargmann, C.I., and Bazzicalupo, P. (2002). C. elegans responds to chemical repellents by integrating sensory inputs from the head and the tail. Curr Biol $12,730-734$.

Hinshaw, J.E. (2000). Dynamin and its role in membrane fission. Annu Rev Cell Dev Biol 16, 483-519.

Hobson, R.J., Liu, Q., Watanabe, S., and Jorgensen, E.M. (2011). Complexin maintains vesicles in the primed state in C. elegans. Curr Biol 21, 106-113.

Hosono, R., Hekimi, S., Kamiya, Y., Sassa, T., Murakami, S., Nishiwaki, K., Miwa, J., Taketo, A., and Kodaira, K.I. (1992). The unc-18 gene encodes a novel protein affecting the kinetics of acetylcholine metabolism in the nematode Caenorhabditis elegans. J Neurochem 58, 1517-1525.

Huntwork, S., and Littleton, J.T. (2007). A complexin fusion clamp regulates spontaneous neurotransmitter release and synaptic growth. Nature Publishing Group $10,1235-1237$.

Hutagalung, A.H., and Novick, P.J. (2011). Role of rab GTPases in membrane traffic and cell physiology. Physiol Rev 91, 119-149.

Itzen, A., and Goody, R.S. (2011). GTPases involved in vesicular trafficking: Structures and mechanisms. Semin Cell Dev Biol 22, 48-56.

Jackson, A.P., Flett, A., Smythe, C., Hufton, L., Wettey, F.R., and Smythe, E. (2003). Clathrin promotes incorporation of cargo into coated pits by activation of the AP2 adaptor micro2 kinase. J Cell Biol 163, 231-236. 
Jagoe, W.N., Lindsay, A.J., Read, R.J., McCoy, A.J., McCaffrey, M.W., and Khan, A.R. (2006). Crystal structure of rab11 in complex with rab11 family interacting protein 2. Structure 14, 1273-1283.

Jahn, R., and Scheller, R.H. (2006). SNAREs--engines for membrane fusion. Nat Rev Mol Cell Biol 7, 631-643.

Jockusch, W.J., Speidel, D., Sigler, A., Sørensen, J.B., Varoqueaux, F., Rhee, J.-S., and Brose, N. (2007). CAPS-1 and CAPS-2 are essential synaptic vesicle priming proteins. Cell 131, 796-808.

Johansson, M., Rocha, N., Zwart, W., Jordens, I., Janssen, L., Kuijl, C., Olkkonen, V.M., and Neefjes, J. (2007). Activation of endosomal dynein motors by stepwise assembly of Rab7-RILP-p150Glued, ORP1L, and the receptor betalll spectrin. J Cell Biol 176, 459-471.

Junutula, J.R., De Maziére, A.M., Peden, A.A., Ervin, K.E., Advani, R.J., van Dijk, S.M., Klumperman, J., and Scheller, R.H. (2004). Rab14 is involved in membrane trafficking between the Golgi complex and endosomes. Mol Biol Cell 15, 2218-2229.

Kamath, R.S., Martinez-Campos, M., Zipperlen, P., Fraser, A.G., and Ahringer, J. (2001). Effectiveness of specific RNA-mediated interference through ingested double-stranded RNA in Caenorhabditis elegans. Genome Biol 2.

Katz, B. (1969). The release of neural transmitter substances. Liverpool University Press.

Kawasaki, M., Nakayama, K., and Wakatsuki, S. (2005). Membrane recruitment of effector proteins by Arf and Rab GTPases. Curr Opin Struct Biol 15, 681-689.

Kim, T., Gondre-Lewis, M.C., Arnaoutova, I., and Peng Loh, Y. (2006). Dense-core secretory granule biogenesis. Physiology Bethesda.

Korenbaum, E., and Rivero, F. (2002). Calponin homology domains at a glance. J Cell Sci 115, 3543-3545.

Kouranti, I., Sachse, M., Arouche, N., Goud, B., and Echard, A. (2006). Rab35 regulates an endocytic recycling pathway essential for the terminal steps of cytokinesis. Curr Biol 16, 1719-1725.

Lackner, M.R., Nurrish, S.J., and Kaplan, J.M. (1999). Facilitation of synaptic transmission by EGL-30 Gq and EGL-8 PLC: DAG binding to UNC-13 is required to stimulate acetylcholine release. Neuron 24, 335-346.

Lamaze, C., Chuang, T.H., Terlecky, L.J., Bokoch, G.M., and Schmid, S.L. (1996). Regulation of receptor-mediated endocytosis by Rho and Rac. Nature 382, 177-179.

Landis, D.M., Hall, A.K., Weinstein, L.A., and Reese, T.S. (1988). The organization of cytoplasm at the presynaptic active zone of a central nervous system synapse.

Neuron 1, 201-209. 
Larance, M., Ramm, G., Stöckli, J., van Dam, E.M., Winata, S., Wasinger, V., Simpson, F., Graham, M., Junutula, J.R., Guilhaus, M., et al. (2005). Characterization of the role of the Rab GTPase-activating protein AS160 in insulin-regulated GLUT4 trafficking. J Biol Chem 280, 37803-37813.

Lee, M.-T.G., Mishra, A., and Lambright, D.G. (2009). Structural mechanisms for regulation of membrane traffic by rab GTPases. Traffic 10, 1377-1389.

Lewis, J.A., Wu, C.H., Berg, H., and Levine, J.H. (1980). The genetics of levamisole resistance in the nematode Caenorhabditis elegans. Genetics 95, 905-928.

Li, C., and Kim, K. (2008). Neuropeptides. WormBook, 1-36.

Li, L., Chin, L.S., Shupliakov, O., Brodin, L., Sihra, T.S., Hvalby, O., Jensen, V., Zheng, D., McNamara, J.O., Greengard, P., et al. (1995). Impairment of synaptic vesicle clustering and of synaptic transmission, and increased seizure propensity, in synapsin I-deficient mice. Proc Natl Acad Sci U S A 92, 9235-9239.

Liewald, J.F., Brauner, M., Stephens, G.J., Bouhours, M., Schultheis, C., Zhen, M., and Gottschalk, A. (2008). Optogenetic analysis of synaptic function. Nature Methods $5,895-902$.

Liewen, H., Meinhold-Heerlein, I., Oliveira, V., Schwarzenbacher, R., Luo, G., Wadle, A., Jung, M., Pfreundschuh, M., and Stenner-Liewen, F. (2005).

Characterization of the human GARP (Golgi associated retrograde protein) complex. Exp Cell Res 306, 24-34.

Lipatova, Z., Tokarev, A.A., Jin, Y., Mulholland, J., Weisman, L.S., and Segev, N. (2008). Direct interaction between a myosin V motor and the Rab GTPases Ypt31/32 is required for polarized secretion. Mol Biol Cell 19, 4177-4187.

Lippé, R., Miaczynska, M., Rybin, V., Runge, A., and Zerial, M. (2001). Functional synergy between Rab5 effector Rabaptin-5 and exchange factor Rabex-5 when physically associated in a complex. Mol Biol Cell 12, 2219-2228.

Liu, Y., Schirra, C., Edelmann, L., Matti, U., Rhee, J., Hof, D., Bruns, D., Brose, N., Rieger, H., Stevens, D.R., et al. (2010). Two distinct secretory vesicle-priming steps in adrenal chromaffin cells. J Cell Biol 190, 1067-1077.

Lou, X., Korogod, N., Brose, N., and Schneggenburger, R. (2008). Phorbol esters modulate spontaneous and $\mathrm{Ca} 2+$-evoked transmitter release via acting on both Munc13 and protein kinase C. J Neurosci 28, 8257-8267.

Low, H.H., and Löwe, J. (2010). Dynamin architecture--from monomer to polymer. Curr Opin Struct Biol 20, 791-798.

Lowe, M. (2010). Structural organization of the Golgi apparatus. Curr Opin Cell Biol.

Lui-Roberts, W.W.Y., Collinson, L.M., Hewlett, L.J., Michaux, G., and Cutler, D.F. (2005). An AP-1/clathrin coat plays a novel and essential role in forming the WeibelPalade bodies of endothelial cells. J Cell Biol 170, 627-636. 
Madison, J.M., Nurrish, S., and Kaplan, J.M. (2005). UNC-13 interaction with syntaxin is required for synaptic transmission. Curr Biol 15, 2236-2242.

Mahoney, T.R., Liu, Q., Itoh, T., Luo, S., Hadwiger, G., Vincent, R., Wang, Z.-W., Fukuda, M., and Nonet, M.L. (2006a). Regulation of synaptic transmission by RAB-3 and RAB-27 in Caenorhabditis elegans. Mol Biol Cell 17, 2617-2625.

Mahoney, T.R., Luo, S., and Nonet, M.L. (2006b). Analysis of synaptic transmission in Caenorhabditis elegans using an aldicarb-sensitivity assay. Nat Protoc 1, 17721777.

Mahoney, T.R., Luo, S., Round, E.K., Brauner, M., Gottschalk, A., Thomas, J.H., and Nonet, M.L. (2008). Intestinal signaling to GABAergic neurons regulates a rhythmic behavior in Caenorhabditis elegans. Proc Natl Acad Sci USA 105, 16350-16355.

Mallard, F., Tang, B.L., Galli, T., Tenza, D., Saint-Pol, A., Yue, X., Antony, C., Hong, W., Goud, B., and Johannes, L. (2002). Early/recycling endosomes-to-TGN transport involves two SNARE complexes and a Rab6 isoform. J Cell Biol 156, 653664.

Mangahas, P.M., Yu, X., Miller, K.G., and Zhou, Z. (2008). The small GTPase Rab2 functions in the removal of apoptotic cells in Caenorhabditis elegans. J Cell Biol 180, 357-373.

Martinez, O., Schmidt, A., Salaméro, J., Hoflack, B., Roa, M., and Goud, B. (1994). The small GTP-binding protein rab6 functions in intra-Golgi transport. J Cell Biol $127,1575-1588$.

Maruyama, I.N., and Brenner, S. (1991). A phorbol ester/diacylglycerol-binding protein encoded by the unc-13 gene of Caenorhabditis elegans. Proc Natl Acad Sci USA $88,5729-5733$.

McIntire, S.L., Jorgensen, E., Kaplan, J., and Horvitz, H.R. (1993). The GABAergic nervous system of Caenorhabditis elegans. Nature 364, 337-341.

Mello, C., and Fire, A. (1995). DNA transformation. Methods Cell Biol 48, 451-482.

Ménasché, G., Pastural, E., Feldmann, J., Certain, S., Ersoy, F., Dupuis, S., Wulffraat, N., Bianchi, D., Fischer, A., Le Deist, F., et al. (2000). Mutations in RAB27A cause Griscelli syndrome associated with haemophagocytic syndrome. Nat Genet 25, 173 176.

Meresse, S., Gorvel, J.P., and Chavrier, P. (1995). The rab7 GTPase resides on a vesicular compartment connected to lysosomes. J Cell Sci 108 ( Pt 11), 3349-3358.

Miaczynska, M., Christoforidis, S., Giner, A., Shevchenko, A., Uttenweiler- Joseph, S., Habermann, B., Wilm, M., Parton, R.G., and Zerial, M. (2004). APPL proteins link Rab5 to nuclear signal transduction via an endosomal compartment. Cell 116, $445-456$. 
Mîinea, C.P., Sano, H., Kane, S., Sano, E., Fukuda, M., Peränen, J., Lane, W.S., and Lienhard, G.E. (2005). AS160, the Akt substrate regulating GLUT4 translocation, has a functional Rab GTPase-activating protein domain. Biochem J 391, 87-93.

Mohrmann, R., de Wit, H., Verhage, M., Neher, E., and Sørensen, J.B. (2010). Fast vesicle fusion in living cells requires at least three SNARE complexes. Science 330, 502-505.

Morvan, J., and Tooze, S.A. (2008). Discovery and progress in our understanding of the regulated secretory pathway in neuroendocrine cells. Histochem Cell Biol 129, 243-252.

Nachury, M.V., Loktev, A.V., Zhang, Q., Westlake, C.J., Peranen, J., Merdes, A., Slusarski, D.C., Scheller, R.H., Bazan, J.F., Sheffield, V.C., et al. (2007). A core complex of BBS proteins cooperates with the GTPase Rab8 to promote ciliary membrane biogenesis. Cell 129, 1201-1213.

Neher, E., and Marty, A. (1982). Discrete changes of cell membrane capacitance observed under conditions of enhanced secretion in bovine adrenal chromaffin cells. Proc Natl Acad Sci USA 79, 6712-6716.

Nobes, C.D., and Hall, A. (1995). Rho, rac, and cdc42 GTPases regulate the assembly of multimolecular focal complexes associated with actin stress fibers, lamellipodia, and filopodia. Cell 81, 53-62.

Nonet, M.L., Staunton, J.E., Kilgard, M.P., Fergestad, T., Hartwieg, E., Horvitz, H.R., Jorgensen, E.M., and Meyer, B.J. (1997). Caenorhabditis elegans rab-3 mutant synapses exhibit impaired function and are partially depleted of vesicles. J Neurosci 17, 8061-8073.

Nordmann, M., Cabrera, M., Perz, A., Bröcker, C., Ostrowicz, C., EngelbrechtVandré, S., and Ungermann, C. (2010). The Mon1-Ccz1 complex is the GEF of the late endosomal Rab7 homolog Ypt7. Curr Biol 20, 1654-1659.

Nuoffer, C., Davidson, H.W., Matteson, J., Meinkoth, J., and Balch, W.E. (1994). A GDP-bound of rab1 inhibits protein export from the endoplasmic reticulum and transport between Golgi compartments. J Cell Biol 125, 225-237.

Ohya, T., Miaczynska, M., Coskun, U., Lommer, B., Runge, A., Drechsel, D., Kalaidzidis, Y., and Zerial, M. (2009). Reconstitution of Rab- and SNARE-dependent membrane fusion by synthetic endosomes. Nature 459, 1091-1097.

Oka, T., and Krieger, M. (2005). Multi-component protein complexes and Golgi membrane trafficking. J Biochem 137, 109-114.

Olkkonen, V.M., Peterson, J.R., Dupree, P., Lutcke, A., Zerial, M., and Simons, K. (1994). Isolation of a mouse cDNA encoding Rab23, a small novel GTPase expressed predominantly in the brain. Gene 138, 207-211.

Ortiz, D., Medkova, M., Walch-Solimena, C., and Novick, P. (2002). Ypt32 recruits the $\operatorname{Sec} 4 p$ guanine nucleotide exchange factor, $\operatorname{Sec} 2 p$, to secretory vesicles; evidence for a Rab cascade in yeast. J Cell Biol 157, 1005-1015. 
Ostermeier, C., and Brunger, A.T. (1999). Structural basis of Rab effector specificity: crystal structure of the small $\mathrm{G}$ protein Rab3A complexed with the effector domain of rabphilin-3A. Cell 96, 363-374.

Owen, D.J., Collins, B.M., and Evans, P.R. (2004). Adaptors for clathrin coats: structure and function. Annu Rev Cell Dev Biol 20, 153-191.

Ozeki, S., Cheng, J., Tauchi-Sato, K., Hatano, N., Taniguchi, H., and Fujimoto, T. (2005). Rab18 localizes to lipid droplets and induces their close apposition to the endoplasmic reticulum-derived membrane. J Cell Sci 118, 2601-2611.

Pan, X., Eathiraj, S., Munson, M., and Lambright, D.G. (2006). TBC-domain GAPs for Rab GTPases accelerate GTP hydrolysis by a dual-finger mechanism. Nature 442, 303-306.

Pang, Z.P., and Südhof, T.C. (2010). Cell biology of Ca2+-triggered exocytosis. Curr Opin Cell Biol 22, 496-505.

Patel, M.R., Lehrman, E.K., Poon, V.Y., Crump, J.G., Zhen, M., Bargmann, C.I., and Shen, K. (2006). Hierarchical assembly of presynaptic components in defined C. elegans synapses. Nat Neurosci 9, 1488-1498.

Pavlos, N.J., Grønborg, M., Riedel, D., Chua, J.J.E., Boyken, J., Kloepper, T.H., Urlaub, H., Rizzoli, S.O., and Jahn, R. (2010). Quantitative analysis of synaptic vesicle Rabs uncovers distinct yet overlapping roles for Rab3a and Rab27b in Ca2+triggered exocytosis. J Neurosci 30, 13441-13453.

Peplowska, K., Markgraf, D.F., Ostrowicz, C.W., Bange, G., and Ungermann, C. (2007). The CORVET tethering complex interacts with the yeast Rab5 homolog Vps21 and is involved in endo-lysosomal biogenesis. Developmental Cell 12, 739750.

Pereira-Leal, J.B., and Seabra, M.C. (2001). Evolution of the rab family of small GTP-binding proteins. Journal of Molecular Biology 313, 889-901.

Perez-Mansilla, B., and Nurrish, S. (2009). Chapter 4 - A Network of G-Protein Signaling Pathways Control Neuronal Activity in C. elegans. Genetic Dissection of Neural Circuits and Behavior 65, 145-192.

Pieribone, V.A., Shupliakov, O., Brodin, L., Hilfiker-Rothenfluh, S., Czernik, A.J., and Greengard, P. (1995). Distinct pools of synaptic vesicles in neurotransmitter release. Nature 375, 493-497.

Pobbati, A.V., Razeto, A., Böddener, M., Becker, S., and Fasshauer, D. (2004). Structural basis for the inhibitory role of tomosyn in exocytosis. J Biol Chem 279, 47192-47200.

Poteryaev, D., Datta, S., Ackema, K., Zerial, M., and Spang, A. (2010). Identification of the Switch in Early-to-Late Endosome Transition. Cell 141, 497-508.

Pucadyil, T.J., and Schmid, S.L. (2009). Conserved functions of membrane active GTPases in coated vesicle formation. Science 325, 1217-1220. 
Ramirez, I.B.-R., and Lowe, M. (2009). Golgins and GRASPs: holding the Golgi together. Semin Cell Dev Biol 20, 770-779.

Reim, K., Mansour, M., Varoqueaux, F., McMahon, H.T., Südhof, T.C., Brose, N., and Rosenmund, C. (2001). Complexins regulate a late step in Ca2+-dependent neurotransmitter release. Cell 104, 71-81.

Reynolds, E.S. (1963). The use of lead citrate at high $\mathrm{pH}$ as an electron-opaque stain in electron microscopy. Journal of Cell Biology 17, 208-213.

Rhee, J.S., Betz, A., Pyott, S., Reim, K., Varoqueaux, F., Augustin, I., Hesse, D., Sudhof, T.C., Takahashi, M., Rosenmund, C., et al. (2002). Beta phorbol ester- and diacylglycerol-induced augmentation of transmitter release is mediated by Munc13s and not by PKCs. Cell 108, 121-133.

Richmond, J. (2005). Synaptic function. WormBook, 1-14.

Richmond, J.E., Weimer, R.M., and Jorgensen, E.M. (2001). An open form of syntaxin bypasses the requirement for UNC-13 in vesicle priming. Nature 412, 338341.

Riddle, D.L.R., Blumenthal, T., Meyer, B.J., and Priess, J.R. (1997). C. elegans II. Cold Spring Harbor Monograph Series 33.

Rink, J., Ghigo, E., Kalaidzidis, Y., and Zerial, M. (2005). Rab conversion as a mechanism of progression from early to late endosomes. Cell 122, 735-749.

Rivera-Molina, F.E., and Novick, P.J. (2009). A Rab GAP cascade defines the boundary between two Rab GTPases on the secretory pathway. Proc Natl Acad Sci USA 106, 14408-14413.

Rizo, J., and Rosenmund, C. (2008). Synaptic vesicle fusion. Nat Struct Mol Biol 15, 665-674.

Roggo, L., Bernard, V., Kovacs, A.L., Rose, A.M., Savoy, F., Zetka, M., Wymann, M.P., and Müller, F. (2002). Membrane transport in Caenorhabditis elegans: an essential role for VPS34 at the nuclear membrane. EMBO J 21, 1673-1683.

Rojas, R., van Vlijmen, T., Mardones, G.A., Prabhu, Y., Rojas, A.L., Mohammed, S., Heck, A.J.R., Raposo, G., van der Sluijs, P., and Bonifacino, J.S. (2008). Regulation of retromer recruitment to endosomes by sequential action of Rab5 and Rab7. J Cell Biol 183, 513-526.

Rostaing, P., Weimer, R.M., Jorgensen, E.M., Triller, A., and Bessereau, J.L. (2004). Preservation of immunoreactivity and fine structure of adult $\mathrm{C}$. elegans tissues using high-pressure freezing. J Histochem Cytochem 52, 1-12.

Rouillé, Y., Duguay, S.J., Lund, K., Furuta, M., Gong, Q., Lipkind, G., Oliva, A.A., Chan, S.J., and Steiner, D.F. (1995). Proteolytic processing mechanisms in the biosynthesis of neuroendocrine peptides: the subtilisin-like proprotein convertases. Front Neuroendocrinol 16, 322-361. 
Sadakata, T., Shinoda, Y., Sekine, Y., Saruta, C., Itakura, M., Takahashi, M., and Furuichi, T. (2010). Interaction of calcium-dependent activator protein for secretion 1 (CAPS1) with the class II ADP-ribosylation factor small GTPases is required for dense-core vesicle trafficking in the trans-Golgi network. J Biol Chem 285, 3871038719 .

Sambrook, J., and Russel, D.W. (2001). Molecular Cloning: A laboratory manual. Cold Spring Harbor Laboratory Press 1.

Sano, H., Eguez, L., Teruel, M.N., Fukuda, M., Chuang, T.D., Chavez, J.A., Lienhard, G.E., and McGraw, T.E. (2007). Rab10, a target of the AS160 Rab GAP, is required for insulin-stimulated translocation of GLUT4 to the adipocyte plasma membrane. Cell Metabolism 5, 293-303.

Sano, H., Roach, William G., Peck, Grantley R., Fukuda, M., and Lienhard, Gustav E. (2008). Rab10 in insulin-stimulated GLUT4 translocation. Biochem J 411, 89.

Sato, M., Sato, K., Liou, W., Pant, S., Harada, A., and Grant, B.D. (2008). Regulation of endocytic recycling by C. elegans Rab35 and its regulator RME-4, a coated-pit protein. EMBO J 27, 1183-1196.

Schafer, W.R. (2005). Egg-laying. WormBook, 1-7.

Schlüter, O.M., Khvotchev, M., Jahn, R., and Südhof, T.C. (2002). Localization versus function of Rab3 proteins. Evidence for a common regulatory role in controlling fusion. J Biol Chem 277, 40919-40929.

Schlüter, O.M., Schmitz, F., Jahn, R., Rosenmund, C., and Südhof, T.C. (2004). A complete genetic analysis of neuronal Rab3 function. J Neurosci 24, 6629-6637.

Schoebel, S., Oesterlin, L.K., Blankenfeldt, W., Goody, R.S., and Itzen, A. (2009). RabGDI displacement by DrrA from Legionella is a consequence of its guanine nucleotide exchange activity. Mol Cell 36, 1060-1072.

Schonn, J.-S., Maximov, A., Lao, Y., Südhof, T.C., and Sørensen, J.B. (2008). Synaptotagmin-1 and -7 are functionally overlapping $\mathrm{Ca} 2+$ sensors for exocytosis in adrenal chromaffin cells. Proc Natl Acad Sci USA 105, 3998-4003.

Schuck, S., Gerl, M.J., Ang, A., Manninen, A., Keller, P., Mellman, I., and Simons, K. (2007). Rab10 is involved in basolateral transport in polarized Madin-Darby canine kidney cells. Traffic 8, 47-60.

Schuske, K., Beg, A.A., and Jorgensen, E.M. (2004). The GABA nervous system in C. elegans. Trends Neurosci 27, 407-414.

Seaman, M.N. (2008). Endosome protein sorting: motifs and machinery. Cell Mol Life Sci 65, 2842-2858.

Seaman, M.N., McCaffery, J.M., and Emr, S.D. (1998). A membrane coat complex essential for endosome-to-Golgi retrograde transport in yeast. J Cell Biol 142, 665681. 
Segev, N. (2011). GTPases in intracellular trafficking: An overview. Semin Cell Dev Biol 22, 1-2.

Semerdjieva, S., Shortt, B., Maxwell, E., Singh, S., Fonarev, P., Hansen, J., Schiavo, G., Grant, B.D., and Smythe, E. (2008). Coordinated regulation of AP2 uncoating from clathrin-coated vesicles by rab5 and hRME-6. J Cell Biol 183, 499-511.

Sheffield, P., Garrard, S., and Derewenda, Z. (1999). Overcoming expression and purification problems of RhoGDI using a family of "parallel" expression vectors. Protein Expr Purif 15, 34-39.

Shi, A., Chen, C.C.-H., Banerjee, R., Glodowski, D., Audhya, A., Rongo, C., and Grant, B.D. (2010). EHBP-1 functions with RAB-10 during endocytic recycling in Caenorhabditis elegans. Mol Biol Cell 21, 2930-2943.

Shin, H.-W., Hayashi, M., Christoforidis, S., Lacas-Gervais, S., Hoepfner, S., Wenk, M.R., Modregger, J., Uttenweiler-Joseph, S., Wilm, M., Nystuen, A., et al. (2005). An enzymatic cascade of Rab5 effectors regulates phosphoinositide turnover in the endocytic pathway. J Cell Biol 170, 607-618.

Sieburth, D., Ch'ng, Q., Dybbs, M., Tavazoie, M., Kennedy, S., Wang, D., Dupuy, D., Rual, J.-F., Hill, D.E., Vidal, M., et al. (2005). Systematic analysis of genes required for synapse structure and function. Nature 436, 510-517.

Sieburth, D., Madison, J.M., and Kaplan, J.M. (2007). PKC-1 regulates secretion of neuropeptides. Nat Neurosci 10, 49-57.

Simonsen, A., Lippé, R., Christoforidis, S., Gaullier, J.M., Brech, A., Callaghan, J., Toh, B.H., Murphy, C., Zerial, M., and Stenmark, H. (1998). EEA1 links PI(3)K function to Rab5 regulation of endosome fusion. Nature 394, 494-498.

Siniossoglou, S., and Pelham, H.R.B. (2002). Vps51p links the VFT complex to the SNARE Tlg1p. J Biol Chem 277, 48318-48324.

Sinka, R., Gillingham, A.K., Kondylis, V., and Munro, S. (2008). Golgi coiled-coil proteins contain multiple binding sites for Rab family G proteins. J Cell Biol 183, 607-615.

Sönnichsen, B., De Renzis, S., Nielsen, E., Rietdorf, J., and Zerial, M. (2000). Distinct membrane domains on endosomes in the recycling pathway visualized by multicolor imaging of Rab4, Rab5, and Rab11. J Cell Biol 149, 901-914.

Stenmark, H. (2009). Rab GTPases as coordinators of vesicle traffic. Nat Rev Mol Cell Biol 10, 513-525.

Stenmark, H., Parton, R.G., Steele-Mortimer, O., Lütcke, A., Gruenberg, J., and Zerial, M. (1994). Inhibition of rab5 GTPase activity stimulates membrane fusion in endocytosis. The EMBO Journal 13, 1287-1296.

Stenmark, H., Vitale, G., Ullrich, O., and Zerial, M. (1995). Rabaptin-5 is a direct effector of the small GTPase Rab5 in endocytic membrane fusion. Cell 83, 423-432. 
Stevens, D.R., and Rettig, J. (2009). The $\mathrm{Ca}(2+)$-dependent activator protein for secretion CAPS: do I dock or do I prime? Mol Neurobiol 39, 62-72.

Stroupe, C., Hickey, C.M., Mima, J., Burfeind, A.S., and Wickner, W. (2009).

Minimal membrane docking requirements revealed by reconstitution of Rab GTPasedependent membrane fusion from purified components. Proc Natl Acad Sci USA 106, 17626-17633.

Sudhof, T.C. (2004). The synaptic vesicle cycle. Annu Rev Neurosci 27, 509-547.

Sumakovic, M., Hegermann, J., Luo, L., Husson, S.J., Schwarze, K., Olendrowitz, C., Schoofs, L., Richmond, J., and Eimer, S. (2009). UNC-108/RAB-2 and its effector RIC-19 are involved in dense core vesicle maturation in Caenorhabditis elegans. The Journal of Cell Biology 186, 897-914.

Suvorova, E.S., Duden, R., and Lupashin, V.V. (2002). The Sec34/Sec35p complex, a Yptlp effector required for retrograde intra-Golgi trafficking, interacts with Golgi SNAREs and COPI vesicle coat proteins. J Cell Biol 157, 631-643.

Symons, M., and Rusk, N. (2003). Control of vesicular trafficking by Rho GTPases. Curr Biol 13, R409-418.

Tabara, H., Grishok, A., and Mello, C.C. (1998). RNAi in C. elegans: soaking in the genome sequence. Science 282, 430-431.

Takai, Y., Sasaki, T., and Matozaki, T. (2001). Small GTP-binding proteins. Physiol Rev 81, 153-208.

Takamori, S., Holt, M., Stenius, K., Lemke, E.A., Grønborg, M., Riedel, D., Urlaub, H., Schenck, S., Brügger, B., Ringler, P., et al. (2006). Molecular anatomy of a trafficking organelle. Cell 127, 831-846.

Terzyan, S., Zhu, G., Li, G., and Zhang, X.C. (2004). Refinement of the structure of human Rab5a GTPase domain at 1.05 A resolution. Acta Crystallogr D Biol Crystallogr 60, 54-60.

Thomas, J.H. (1990). Genetic analysis of defecation in Caenorhabditis elegans. Genetics 124, 855-872.

Timmons, L. (2004). Endogenous inhibitors of RNA interference in Caenorhabditis elegans. Bioessays 26, 715-718.

Tolmachova, T., Abrink, M., Futter, C.E., Authi, K.S., and Seabra, M.C. (2007). Rab27b regulates number and secretion of platelet dense granules. Proc Natl Acad Sci USA $104,5872-5877$.

Tong, Y.-G., and Bürglin, T.R. (2010). Conditions for dye-filling of sensory neurons in Caenorhabditis elegans. J Neurosci Methods 188, 58-61.

Tooze, S.A., Flatmark, T., Tooze, J., and Huttner, W.B. (1991). Characterization of the immature secretory granule, an intermediate in granule biogenesis. J Cell Biol $115,1491-1503$. 
Tooze, S.A., Martens, G.J., and Huttner, W.B. (2001). Secretory granule biogenesis: rafting to the SNARE. Trends Cell Biol 11, 116-122.

Touchot, N., Chardin, P., and Tavitian, A. (1987). Four additional members of the ras gene superfamily isolated by an oligonucleotide strategy: molecular cloning of YPTrelated cDNAs from a rat brain library. Proc Natl Acad Sci USA 84, 8210-8214.

Trajkovski, M., Mziaut H., Schubert X., Kalaidzidis Y., Altkruger A., and Solimena M. (2008). Regulation of insulin granule turnover in pancreatic beta-cells by cleaved ICA512. J Biol Chem. 283:33719-29.

Ueno, H., Huang, X., Tanaka, Y., and Hirokawa, N. (2011). KIF16B/Rab14 molecular motor complex is critical for early embryonic development by transporting FGF receptor. Developmental Cell 20, 60-71.

Ullrich, O., Reinsch, S., Urbé, S., Zerial, M., and Parton, R.G. (1996). Rab11 regulates recycling through the pericentriolar recycling endosome. J Cell Biol 135, 913-924.

Van Gele, M., Dynoodt, P., and Lambert, J. (2009). Griscelli syndrome: a model system to study vesicular trafficking. Pigment Cell Melanoma Res 22, 268-282.

Villanueva, M., Thornley, K., Augustine, G.J., and Wightman, R.M. (2006). Synapsin II negatively regulates catecholamine release. Brain cell biology 35, 125-136.

Voets, T. (2000). Dissection of three Ca2+-dependent steps leading to secretion in chromaffin cells from mouse adrenal slices. Neuron 28, 537-545.

Voets, T., Toonen, R.F., Brian, E.C., de Wit, H., Moser, T., Rettig, J., Südhof, T.C., Neher, E., and Verhage, M. (2001). Munc18-1 promotes large dense-core vesicle docking. Neuron 31, 581-591.

von Stetina, S.E., Treinin, M., and Miller, D.M. (2006). The Motor Circuit. Intl Review of Neurobiology 69, 1-43.

von Stetina, S.E., Watson, J.D., Fox, R.M., Olszewski, K.L., Spencer, W.C., Roy, P.J., and Miller, D.M. (2007). Cell-specific microarray profiling experiments reveal a comprehensive picture of gene expression in the $\mathrm{C}$. elegans nervous system. Genome Biol 8, R135.

Waggoner, L.E., Zhou, G.T., Schafer, R.W., and Schafer, W.R. (1998). Control of alternative behavioral states by serotonin in Caenorhabditis elegans. Neuron 21, 203214.

Wagner, W., Bielli, P., Wacha, S., and Ragnini-Wilson, A. (2002). Mlc1p promotes septum closure during cytokinesis via the IQ motifs of the vesicle motor Myo2p.

EMBO J 21, 6397-6408.

Walent, J.H., Porter, B.W., and Martin, T.F. (1992). A novel 145 kd brain cytosolic protein reconstitutes $\mathrm{Ca}(2+)$-regulated secretion in permeable neuroendocrine cells. Cell 70, 765-775. 
Watanabe, M., Nomura, K., Ohyama, A., Ishikawa, R., Komiya, Y., Hosaka, K., Yamauchi, E., Taniguchi, H., Sasakawa, N., Kumakura, K., et al. (2005). Myosin-Va regulates exocytosis through the submicromolar $\mathrm{Ca} 2+$-dependent binding of syntaxin1A. Mol Biol Cell 16, 4519-4530.

Weber, T., Zemelman, B.V., McNew, J.A., Westermann, B., Gmachl, M., Parlati, F., Söllner, T.H., and Rothman, J.E. (1998). SNAREpins: minimal machinery for membrane fusion. Cell 92, 759-772.

Wendler, F., Page, L., Urbé, S., and Tooze, S.A. (2001). Homotypic fusion of immature secretory granules during maturation requires syntaxin 6. Mol Biol Cell 12, 1699-1709.

Wennerberg, K., Rossman, K.L., and Der, C.J. (2005). The Ras superfamily at a glance. Journal of Cell Science 118, 843-846.

Wicks, S. (2000). CHE-3, a Cytosolic Dynein Heavy Chain, Is Required for Sensory Cilia Structure and Function in Caenorhabditis elegans. Developmental Biology 221, 295-307.

Wu, M., Wang, T., Loh, E., Hong, W., and Song, H. (2005). Structural basis for recruitment of RILP by small GTPase Rab7. The EMBO Journal 24, 1491-1501.

Wu, X.S., Rao, K., Zhang, H., Wang, F., Sellers, J.R., Matesic, L.E., Copeland, N.G., Jenkins, N.A., and Hammer, J.A. (2002). Identification of an organelle receptor for myosin-Va. Nat Cell Biol 4, 271-278.

Wurmser, A.E., Sato, T.K., and Emr, S.D. (2000). New component of the vacuolar class C-Vps complex couples nucleotide exchange on the Ypt7 GTPase to SNAREdependent docking and fusion. J Cell Biol 151, 551-562.

Xue, M., Reim, K., Chen, X., Chao, H.T., Deng, H., Rizo, J., Brose, N., and Rosenmund, C. (2007). Distinct domains of complexin I differentially regulate neurotransmitter release. Nat Struct Mol Biol 14, 949-958.

Yamasaki, A., Menon, S., Yu, S., Barrowman, J., Meerloo, T., Oorschot, V., Klumperman, J., Satoh, A., and Ferro-Novick, S. (2009). mTrs130 is a component of a mammalian TRAPPII complex, a Rab1 GEF that binds to COPI-coated vesicles. Mol Biol Cell 20, 4205-4215.

Yang, X., Kaeser-Woo, Y.J., Pang, Z.P., Xu, W., and Südhof, T.C. (2010). Complexin clamps asynchronous release by blocking a secondary $\mathrm{Ca}(2+)$ sensor via its accessory $\alpha$ helix. Neuron $68,907-920$.

Yizhar, O., Matti, U., Melamed, R., Hagalili, Y., Bruns, D., Rettig, J., and Ashery, U. (2004). Tomosyn inhibits priming of large dense-core vesicles in a calcium-dependent manner. Proc Natl Acad Sci USA 101, 2578-2583.

Yoshimura, S.-i., Gerondopoulos, A., Linford, A., Rigden, D.J., and Barr, F.A. (2010). Family-wide characterization of the DENN domain Rab GDP-GTP exchange factors. J Cell Biol 191, 367-381. 
Yu, E., Kanno, E., Choi, S., Sugimori, M., Moreira, J.E., Llinás, R.R., and Fukuda, M. (2008). Role of Rab27 in synaptic transmission at the squid giant synapse. Proc Natl Acad Sci USA 105, 16003-16008.

Yu, I.-M., and Hughson, F.M. (2010). Tethering factors as organizers of intracellular vesicular traffic. Annu Rev Cell Dev Biol 26, 137-156.

Zerial, M., and McBride, H. (2001). Rab proteins as membrane organizers. Nat Rev Mol Cell Biol 2, 107-117.

Zhang, J., Schulze, K.L., Hiesinger, P.R., Suyama, K., Wang, S., Fish, M., Acar, M., Hoskins, R.A., Bellen, H.J., and Scott, M.P. (2007). Thirty-one flavors of Drosophila rab proteins. Genetics 176, 1307-1322.

Zhang, Y., Grant, B., and Hirsh, D. (2001). RME-8, a conserved J-domain protein, is required for endocytosis in Caenorhabditis elegans. Mol Biol Cell 12, 2011-2021.

Zhang, Z., Bhalla, A., Dean, C., Chapman, E.R., and Jackson, M.B. (2009). Synaptotagmin IV: a multifunctional regulator of peptidergic nerve terminals. Nat Neurosci 12, 163-171.

Zhao, B., Wang, H.-B., Lu, Y.-J., Hu, J.-W., Bao, L., and Zhang, X. (2011). Transport of receptors, receptor signaling complexes and ion channels via neuropeptidesecretory vesicles. Cell Research, 1-13.

Zhu, G., Zhai, P., Liu, J., Terzyan, S., Li, G., and Zhang, X.C. (2004). Structural basis of Rab5-Rabaptin5 interaction in endocytosis. Nat Struct Mol Biol 11, 975-983. 


\section{APPENDIX}

\subsection{Constructs and bacteria/yeast strains}

All bacterial/yeast strains and constructs used in this study are listed in table XII.1. A brief description is provided about all the backbone vectors used as well as the cloning strategies used for the generation of new vectors.

Table XII.1. List of all bacterial/yeast strains and constructs used in study

\begin{tabular}{|c|c|c|c|}
\hline $\begin{array}{l}\text { Bacteria and Yeast } \\
\text { Strains }\end{array}$ & \multicolumn{2}{|l|}{ Description } & Source \\
\hline DH5 $\alpha$ & \multicolumn{2}{|c|}{ E.coli strain used for general molecular cloning } & Invitrogen \\
\hline DB3.1 & \multicolumn{2}{|c|}{$\begin{array}{l}\text { E.coli strain used for molecular cloning of vectors } \\
\text { containing the } c c d \mathrm{~B} \text { gene. Contains the gyrA } 462 \\
\text { allele, which confers resistance to } c c d \mathrm{~B} \text {. For gateway } \\
\text { cloning }\end{array}$} & Invitrogen \\
\hline B121-Star (DE3) & \multicolumn{2}{|c|}{$\begin{array}{l}\text { E.coli Strain for protein expression. DE } 3 \text { indicate } \\
\text { that the expression of } \mathrm{T} 7 \text { polymerase is inducible by } \\
\text { IPTG. Contains additional mutation in rne131, an } \\
\text { RNAase. }\end{array}$} & Invitrogen \\
\hline HT115 (DE3) & \multicolumn{2}{|c|}{$\begin{array}{l}\text { E.coli } \text { Sttrain for feeding induced RNAi in C.elegans. } \\
\text { Double stranded RNA induced by IPTG }\end{array}$} & $\begin{array}{l}\text { Kamath et al., } \\
2001\end{array}$ \\
\hline OP50 & \multicolumn{2}{|c|}{ E.coil strain for feeding C.elegans } & Brenner, 1974 \\
\hline AH109 & \multicolumn{2}{|c|}{ S.cerevisae strain used for yeast-2-hybrid } & Clontech \\
\hline Backbone Vectors & $\begin{array}{l}\text { Resistance } \\
\text { Gene }\end{array}$ & Description & Source \\
\hline pBluescript SK (+) & $A m p^{r}$ & Entry vector used for cloning & Stratagene \\
\hline pENTR4 & $\mathrm{Kan}^{\mathrm{r}}$ & Gateway entry vector & Invitrogen \\
\hline pGEMT & $\mathrm{Amp}^{\mathrm{r}}$ & Entry vector for TA cloning & Promega \\
\hline $\begin{array}{l}\text { pPD1 15.62(pmyo- } \\
3: \because g f p)\end{array}$ & $A m p^{r}$ & $\begin{array}{l}\text { Muscle expression vector for C.elegans } \\
\text { Contains } u n c-543 U ́ T R\end{array}$ & $\begin{array}{l}\text { Andrew Fire } \\
\text { vector kit }\end{array}$ \\
\hline $\begin{array}{l}\text { phsp- } \\
16.41: \because \operatorname{tag} R F P\end{array}$ & $A m p^{r}$ & $\begin{array}{l}\text { Heat shock induced expression vector } \\
\text { for } \\
\text { C.elegans. Based on pPD } 115.62\end{array}$ & $\begin{array}{l}\text { Kindly provided by } \\
\text { Katrin Schwarze } \\
\text { and Sabine Koenig }\end{array}$ \\
\hline prab-3::mcherry & $A m p^{r}$ & $\begin{array}{l}\text { Neuronal expression vector } \\
\text { for C.elegans. Based on pPD115.62 }\end{array}$ & $\begin{array}{l}\text { Kindly provided by } \\
\text { Marija Sumakovic }\end{array}$ \\
\hline prab-3::tagRFP & $A m p^{r}$ & $\begin{array}{l}\text { Neuronal expression vector } \\
\text { for C.elegans. Based on pPD } 115.62\end{array}$ & $\begin{array}{l}\text { Kindly provided by } \\
\text { Marija Sumakovic }\end{array}$ \\
\hline prab-3::gfp & $A m p^{r}$ & $\begin{array}{l}\text { Neuronal expression vector } \\
\text { for C.elegans. Based on pPD } 115.62\end{array}$ & $\begin{array}{l}\text { Kindly provided by } \\
\text { Marija Sumakovic }\end{array}$ \\
\hline punc-129::mcherry & $\mathrm{Amp}^{\mathrm{r}}$ & $\begin{array}{l}\text { DA and DB motoneuron expression } \\
\text { vector for C.elegans. } \\
\text { Based on prab-3::mcherry }\end{array}$ & $\begin{array}{l}\text { Kindly provided by } \\
\text { Marija Sumakovic }\end{array}$ \\
\hline L4440 & $A m p^{r}$ & Entry vector for feeding RNAi & $\begin{array}{l}\text { Kindly provided by } \\
\text { Julie Ahringer }\end{array}$ \\
\hline pGST parallel 2 & $A m p^{r}$ & $\begin{array}{l}\text { GST-Protein expression vector based } \\
\text { on pGEX4T1 }\end{array}$ & $\begin{array}{l}\text { Sheffield et al., } \\
1999\end{array}$ \\
\hline pGBKT7 & $\mathrm{Kan}^{\mathrm{r}}$ & $\begin{array}{l}\text { Yeast-2-hybrid vector containing } \\
\text { DNA binding domain }\end{array}$ & Clontech \\
\hline pGADT7 & $A m p^{r}$ & $\begin{array}{l}\text { Yeast-2-hybrid vector containing } \\
\text { DNA activating domain }\end{array}$ & Clontech \\
\hline
\end{tabular}




\begin{tabular}{|c|c|c|c|}
\hline $\begin{array}{l}\text { Genes of interest } \\
\text { in entry vectors }\end{array}$ & $\begin{array}{l}\text { Resistance } \\
\text { Gene }\end{array}$ & Cloning Strategy & Source \\
\hline pBluescript $r a b-1$ & $A m p^{r}$ & -- & $\begin{array}{l}\text { Kindly provided by } \\
\text { Katrin Schwarze }\end{array}$ \\
\hline pBluescript $r a b-2$ & $\mathrm{Amp}^{\mathrm{r}}$ & -- & $\begin{array}{l}\text { Kindly provided by } \\
\text { Katrin Schwarze }\end{array}$ \\
\hline pGEMT $r a b-3$ & $\mathrm{Amp}^{\mathrm{r}}$ & $\begin{array}{l}\text { PCR with oGQ230/oGQ231 } \\
\text { TA cloning }\end{array}$ & Cloned in this study \\
\hline pENTR4 $r a b-5$ & $\mathrm{Kan}^{\mathrm{r}}$ & $\begin{array}{l}\text { PCR with oGQ285/oGQ286 } \\
\text { Digested with BamH1/EcoRI }\end{array}$ & Cloned in this study \\
\hline pENTR4 rab-6.1 & $\operatorname{Kan}^{\mathrm{r}}$ & -- & $\begin{array}{l}\text { Kindly provided by } \\
\text { Katrin Schwarze }\end{array}$ \\
\hline pENTR4 $r a b-6.2$ & $\operatorname{Kan}^{\mathrm{r}}$ & -- & $\begin{array}{l}\text { Kindly Provided by } \\
\text { Katrin Schwarze }\end{array}$ \\
\hline pENTR4 $r a b-7$ & $\operatorname{Kan}^{\mathrm{r}}$ & $\begin{array}{l}\text { PCR with oGQ283/oGQ284 } \\
\text { Digested with BamH1/Xho1 }\end{array}$ & Cloned in this study \\
\hline pENTR4 $r a b-8$ & $\operatorname{Kan}^{\mathrm{r}}$ & $\begin{array}{l}\text { PCR with oGQ259/oGQ260 } \\
\text { Digested with BamH/EcoR1 }\end{array}$ & Cloned in this study \\
\hline pENTR4 rab-10 & $\operatorname{Kan}^{\mathrm{r}}$ & $\begin{array}{l}\text { PCR with oGQ261/oGQ262 } \\
\text { Digested with BamH1/EcoR1 }\end{array}$ & Cloned in this study \\
\hline pGEMT $r a b-11.1$ & $\mathrm{Amp}^{\mathrm{r}}$ & $\begin{array}{l}\text { PCR with oGQ1127/oGQ1128TA } \\
\text { cloning }\end{array}$ & Cloned in this study \\
\hline pENTR4 $r a b-11.1$ & $\mathrm{Kan}^{\mathrm{r}}$ & -- & $\begin{array}{l}\text { Kindly provided by } \\
\text { Katrin Schwarze }\end{array}$ \\
\hline pGEMT $r a b-11.2$ & $\mathrm{Amp}^{\mathrm{r}}$ & $\begin{array}{l}\text { PCR with oGQ1129/oGQ1130 } \\
\text { TA cloning }\end{array}$ & Cloned in this study \\
\hline pBluescript $r a b-14$ & $A m p^{r}$ & -- & $\begin{array}{l}\text { Kindly provided by } \\
\text { Katrin Schwarze }\end{array}$ \\
\hline pENTR4 $r a b-18$ & $\mathrm{Kan}^{\mathrm{r}}$ & $\begin{array}{l}\text { PCR with oGQ268/oGQ269 } \\
\text { Digested with EcoRV/EcoRI }\end{array}$ & Cloned in this study \\
\hline pBluescript $r a b-18$ & $A m p^{r}$ & $\begin{array}{l}\text { Subcloned from pENTR4 rab-18 } \\
\text { Digested with Nde1/Xho1 }\end{array}$ & Cloned in this study \\
\hline pENTR4 rab-19 & $\mathrm{Kan}^{\mathrm{r}}$ & $\begin{array}{l}\text { PCR with oGQ590/oGQ591 } \\
\text { Digested with EcoRV/EcoRI }\end{array}$ & Cloned in this study \\
\hline pENTR4 $r a b-21$ & $\mathrm{Kan}^{\mathrm{r}}$ & $\begin{array}{l}\text { PCR with oGQ263/oGQ264 } \\
\text { Digested with BamH1/EcoR1 }\end{array}$ & Cloned in this study \\
\hline pENTR4 $r a b-27$ & $\mathrm{Kan}^{\mathrm{r}}$ & $\begin{array}{l}\text { PCR with oGQ265/oGQ266 } \\
\text { Digested with BamH1/Xho1 }\end{array}$ & Cloned in this study \\
\hline pENTR4 $r a b-28$ & $\mathrm{Kan}^{\mathrm{r}}$ & $\begin{array}{l}\text { PCR with oGQ277/oGQ278 } \\
\text { Digested with EcoRV/EcoR1 }\end{array}$ & Cloned in this study \\
\hline pBluesscript $r a b-28$ & $A m p^{r}$ & $\begin{array}{l}\text { PCR with oGQ851/oGQ278 } \\
\text { Digested with Not1/EcoR1 }\end{array}$ & Cloned in this study \\
\hline pENTR4 rab-30 & $\mathrm{Kan}^{\mathrm{r}}$ & $\begin{array}{l}\text { PCR with oGQ281/oGQ282 } \\
\text { Digested with EcoRV/Xho1 }\end{array}$ & Cloned in this study \\
\hline pBluescript $r a b-30$ & $A m p^{r}$ & $\begin{array}{l}\text { PCR with oGQ852/oGQ282 } \\
\text { Digested with Not1/Xho1 }\end{array}$ & Cloned in this study \\
\hline pENTR4 rab-33 & $\mathrm{Kan}^{\mathrm{r}}$ & $\begin{array}{l}\text { PCR with oGQ 279/oGQ280 } \\
\text { Digested with Nde1/EcoR1 }\end{array}$ & Cloned in this study \\
\hline pBluescript $r a b-33$ & $A m p^{r}$ & $\begin{array}{l}\text { Subcloned from pENTR4 rab-33 } \\
\text { Digested with Nde1/Xho1 }\end{array}$ & Cloned in this study \\
\hline pENTR4 $r a b-35$ & $\operatorname{Kan}^{r}$ & $\begin{array}{l}\text { PCR with oGQ272/oGQ273 } \\
\text { Digested with BamH1/EcoR1 }\end{array}$ & Cloned in this study \\
\hline pENTR4 $r a b-37.1$ & $\operatorname{Kan}^{\mathrm{r}}$ & $\begin{array}{l}\text { PCR with oGQ274/oGQ275 } \\
\text { Digested with BamH1/EcoR1 }\end{array}$ & Cloned in this study \\
\hline pENTR4 $r a b-37.2$ & $\operatorname{Kan}^{r}$ & $\begin{array}{l}\text { PCR with oGQ276/oGQ275 } \\
\text { Digested with BamH1/EcoR1 }\end{array}$ & Cloned in this study \\
\hline
\end{tabular}




\begin{tabular}{|c|c|c|c|}
\hline pENTR4 rab-39 & $\operatorname{Kan}^{\mathrm{r}}$ & $\begin{array}{l}\text { PCR with oGQ270/oGQ271 } \\
\text { Digested with EcoRV/Xho1 }\end{array}$ & Cloned in this study \\
\hline pGEMT $r a b-y 1$ & $A m p^{r}$ & $\begin{array}{l}\text { PCR with oGQ1343/oGQ1344 } \\
\text { TA cloning }\end{array}$ & Cloned in this study \\
\hline pENTR4 $r a b-y 1$ & $\operatorname{Kan}^{\mathrm{r}}$ & $\begin{array}{l}\text { Subcloned from pGEMT rab-y1 } \\
\text { Digested with Age1/Xho1 }\end{array}$ & Cloned in this study \\
\hline pENTR4 $r a b-y 2$ & $\operatorname{Kan}^{\mathrm{r}}$ & $\begin{array}{l}\text { PCR with oGQ640/oGQ641 } \\
\text { Digested with EcoRV/Xho1 }\end{array}$ & Cloned in this study \\
\hline pENTR4 $r a b-y 3$ & $\operatorname{Kan}^{\mathrm{r}}$ & $\begin{array}{l}\text { PCR with oGQ642/oGQ643 } \\
\text { Digested with EcoRV/EcoR1 }\end{array}$ & Cloned in this study \\
\hline pGEMT $r a b-y 6$ & $A m p^{r}$ & $\begin{array}{l}\text { PCR with oGQ648/oGQ649 } \\
\text { TA cloning }\end{array}$ & Cloned in this study \\
\hline pENTR4 rab-y6 & $\operatorname{Kan}^{\mathrm{r}}$ & $\begin{array}{l}\text { Subcloned from pGEMT rab-y6 } \\
\text { Digested with BamH1/EcoR1 }\end{array}$ & Cloned in this study \\
\hline pGEMT glo-1 & $A m p^{r}$ & $\begin{array}{l}\text { PCR with oGQ1176/oGQ1177 } \\
\text { TA cloning }\end{array}$ & Cloned in this study \\
\hline $\begin{array}{l}\text { Genes of interest } \\
\text { in RNAi vectors }\end{array}$ & $\begin{array}{l}\text { Resistance } \\
\text { Gene }\end{array}$ & Cloning Strategy & Source \\
\hline L4440 $r a b-1$ & $A m p^{r}$ & LR Gateway cloning & $\begin{array}{l}\text { Kindly provided by } \\
\text { Katrin Schwarze }\end{array}$ \\
\hline L4440 $r a b-2$ & $A m p^{r}$ & LR Gateway cloning & $\begin{array}{l}\text { Kindly provided by } \\
\text { Marija Sumakovic }\end{array}$ \\
\hline L4440 rab-3 & $A m p^{r}$ & LR Gateway cloning & $\begin{array}{l}\text { Kindly provided by } \\
\text { Marija Sumakovic }\end{array}$ \\
\hline L4440 $r a b-5$ & $A m p^{r}$ & LR Gateway cloning & Cloned in this study \\
\hline L4440 $r a b-6.1$ & $A m p^{r}$ & LR Gateway cloning & Cloned in this study \\
\hline L4440 $r a b-6.2$ & $A m p^{r}$ & LR Gateway cloning & Cloned in this study \\
\hline L4440 $r a b-7$ & $A m p^{r}$ & LR Gateway cloning & Cloned in this study \\
\hline L4440 $r a b-8$ & $A m p^{r}$ & LR Gateway cloning & Cloned in this study \\
\hline L4440 rab-10 & $A m p^{r}$ & LR Gateway cloning & Cloned in this study \\
\hline L4440 rab-11.1 & $A m p^{r}$ & LR Gateway cloning & Cloned in this study \\
\hline L4440 rab-11.2 & $A m p^{r}$ & $\begin{array}{l}\text { Subcloned from pGEMT rab-11.2 } \\
\text { Digested with Age1/Xho1 }\end{array}$ & Cloned in this study \\
\hline L4440 rab-14 & $A m p^{r}$ & LR Gateway cloning & Cloned in this study \\
\hline L4440 rab-18 & $A m p^{r}$ & LR Gateway cloning & Cloned in this study \\
\hline L4440 $r a b-19$ & $A m p^{r}$ & LR Gateway cloning & Cloned in this study \\
\hline L4440 rab-21 & $A m p^{r}$ & LR Gateway cloning & Cloned in this study \\
\hline L4440 rab-27 & $A m p^{r}$ & LR Gateway cloning & Cloned in this study \\
\hline L4440 $r a b-28$ & $A m p^{r}$ & LR Gateway cloning & Cloned in this study \\
\hline L4440 rab-30 & $A m p^{r}$ & LR Gateway cloning & Cloned in this study \\
\hline L4440 rab-33 & $A m p^{r}$ & LR Gateway cloning & Cloned in this study \\
\hline L4440 $r a b-35$ & $A m p^{r}$ & LR Gateway cloning & Cloned in this study \\
\hline L4440 rab-37.1 & $A m p^{r}$ & LR Gateway cloning & Cloned in this study \\
\hline L4440 rab-37.2 & $A m p^{r}$ & LR Gateway cloning & Cloned in this study \\
\hline L4440 rab-39 & $A m p^{r}$ & LR Gateway cloning & Cloned in this study \\
\hline L4440 rab-y1 & $A m p^{r}$ & $\begin{array}{l}\text { Subcloned from pGEMT rab-y1 } \\
\text { Digested with Age1/Xho1 }\end{array}$ & Cloned in this study \\
\hline L4440 rab-y2 & $A m p^{r}$ & LR Gateway cloning & Cloned in this study \\
\hline L4440 $r a b-y 3$ & $A m p^{r}$ & LR Gateway cloning & Cloned in this study \\
\hline L4440 rab-y6 & $A m p^{r}$ & LR Gateway cloning & Cloned in this study \\
\hline L4440 glo- 1 & $A m p^{r}$ & $\begin{array}{l}\text { Subcloned from pGEMT glo-1 } \\
\text { Digested with Age1/Xho1 }\end{array}$ & Cloned in this study \\
\hline $\mathrm{L} 4440 t b c-4$ & $A m p^{r}$ & $\begin{array}{l}\text { PCR with oGQ1590/oGQ1697 } \\
\text { Digested with Age1/Xho1 }\end{array}$ & Cloned in this study \\
\hline
\end{tabular}




\begin{tabular}{|c|c|c|c|}
\hline L4440 eеa-1 & $A m p^{r}$ & $\begin{array}{l}\text { PCR with oGQ941/oGQ942 } \\
\text { Digested with Age1/Xho1 }\end{array}$ & Cloned in this study \\
\hline L4440 ehbp-1 & $A m p^{r}$ & $\begin{array}{l}\text { PCR with oGQ1583/oGQ1584 } \\
\text { Digested with Age1/Xho1 }\end{array}$ & Cloned in this study \\
\hline L4440 rabn-5 & $A m p^{r}$ & $\begin{array}{l}\text { PCR with oGQ478/oGQ479 } \\
\text { Digested with Age1/EcoR1 }\end{array}$ & Cloned in this study \\
\hline L4440 GFP & $A m p^{r}$ & -- & $\begin{array}{l}\text { Kindly provided by } \\
\text { Marija Sumakovic }\end{array}$ \\
\hline $\begin{array}{l}\text { Genes of interest } \\
\text { in protein } \\
\text { expression vectors }\end{array}$ & $\begin{array}{l}\text { Resistance } \\
\text { Gene }\end{array}$ & Cloning Strategy & Source \\
\hline $\begin{array}{l}\text { pGST parallel } 2 \\
\text { rab-1 }\end{array}$ & $A m p^{r}$ & -- & $\begin{array}{l}\text { Kindly provided by } \\
\text { Katrin Schwarze }\end{array}$ \\
\hline $\begin{array}{l}\text { pGST parallel } 2 \\
r a b-2\end{array}$ & $A m p^{r}$ & -- & $\begin{array}{l}\text { Kindly provided by } \\
\text { Marija Sumakovic }\end{array}$ \\
\hline $\begin{array}{l}\text { pGST parallel } 2 \\
\text { rab-3 }\end{array}$ & $\mathrm{Amp}^{\mathrm{r}}$ & -- & $\begin{array}{l}\text { Kindly provided by } \\
\text { Katrin Schwarze }\end{array}$ \\
\hline $\begin{array}{l}\text { pGST parallel } 2 \\
\text { rab-5 }\end{array}$ & $A m p^{r}$ & -- & $\begin{array}{l}\text { Kindly provided by } \\
\text { Katrin Schwarze }\end{array}$ \\
\hline $\begin{array}{l}\text { pGST parallel } 2 \\
r a b-6.1\end{array}$ & $A m p^{r}$ & -- & $\begin{array}{l}\text { Kindly provided by } \\
\text { Katrin Schwarze }\end{array}$ \\
\hline $\begin{array}{l}\text { pGST parallel } 2 \\
\text { rab-6.2 }\end{array}$ & $A m p^{r}$ & -- & $\begin{array}{l}\text { Kindly provided by } \\
\text { Katrin Schwarze }\end{array}$ \\
\hline $\begin{array}{l}\text { pGST parallel } 2 \\
r a b-8\end{array}$ & $\mathrm{Amp}^{\mathrm{r}}$ & $\begin{array}{l}\text { Subcloned from pENTR4 } r a b-8 \\
\text { Digested with BamH1/EcoR1 }\end{array}$ & Cloned in this study \\
\hline $\begin{array}{l}\text { pGST parallel } 2 \\
r a b-10\end{array}$ & $A m p^{r}$ & $\begin{array}{l}\text { Subcloned from pENTR4 rab-10 } \\
\text { Digested with BamH1/EcoR1 }\end{array}$ & Cloned in this study \\
\hline $\begin{array}{l}\text { pGST parallel } 2 \\
\mathrm{rab}-11.1\end{array}$ & $\mathrm{Amp}^{\mathrm{r}}$ & $\begin{array}{l}\text { Subcloned from pENTR4 rab- } \\
\text { 11.1Digested with Nco1/Xho1 }\end{array}$ & Cloned in this study \\
\hline $\begin{array}{l}\text { pGST parallel } 2 \\
\text { rab-14 }\end{array}$ & $A m p^{r}$ & -- & $\begin{array}{l}\text { Kindly provided by } \\
\text { Katrin Schwarze }\end{array}$ \\
\hline $\begin{array}{l}\text { pGST parallel } 2 \\
\text { rab-18 }\end{array}$ & $A m p^{r}$ & $\begin{array}{l}\text { Subcloned from pBluescript } r a b-18 \\
\text { Digested with Nco1/Xho1 }\end{array}$ & Cloned in this study \\
\hline $\begin{array}{l}\text { pGST parallel } 2 \\
r a b-19\end{array}$ & $A m p^{r}$ & $\begin{array}{l}\text { Subcloned from pENTR4 rab-19 } \\
\text { Digested with Nde1/Xho1 }\end{array}$ & Cloned in this study \\
\hline $\begin{array}{l}\text { pGST parallel } 2 \\
r a b-21\end{array}$ & $A m p^{r}$ & $\begin{array}{l}\text { Subcloned from pENTR4 rab-21 } \\
\text { Digested with BamH1/EcoR1 }\end{array}$ & Cloned in this study \\
\hline $\begin{array}{l}\text { pGST parallel } 2 \\
r a b-27\end{array}$ & $A m p^{r}$ & $\begin{array}{l}\text { Subcloned from pENTR4 rab-27 } \\
\text { Digested with BamH1/Xho1 }\end{array}$ & Cloned in this study \\
\hline $\begin{array}{l}\text { pGST parallel } 2 \\
r a b-28\end{array}$ & $A m p^{r}$ & $\begin{array}{l}\text { Subcloned from pENTR4 rab-28 } \\
\text { Digested with BamH1/EcoR1 }\end{array}$ & Cloned in this study \\
\hline $\begin{array}{l}\text { pGST parallel } 2 \\
r a b-30\end{array}$ & $A m p^{r}$ & $\begin{array}{l}\text { Subcloned from pENTR4 rab-30 } \\
\text { Digested with BamH1/Xho1 }\end{array}$ & Cloned in this study \\
\hline $\begin{array}{l}\text { pGST parallel } 2 \\
r a b-33\end{array}$ & $A m p^{r}$ & $\begin{array}{l}\text { Subcloned from pBluescript } r a b-33 \\
\text { Digested with Nco1/Xho1 }\end{array}$ & Cloned in this study \\
\hline $\begin{array}{l}\text { pGST parallel } 2 \\
r a b-35\end{array}$ & $\mathrm{Amp}^{\mathrm{r}}$ & $\begin{array}{l}\text { Subcloned from pENTR4 rab-35 } \\
\text { Digested with BamH1/EcoR1 }\end{array}$ & Cloned in this study \\
\hline $\begin{array}{l}\text { pGST parallel } 2 \\
r a b-37.1\end{array}$ & $A m p^{r}$ & $\begin{array}{l}\text { Subcloned from pENTR4 rab-37.1 } \\
\text { Digested with BamH1/EcoR1 }\end{array}$ & Cloned in this study \\
\hline $\begin{array}{l}\text { pGST parallel } 2 \\
r a b-37.2\end{array}$ & $A m p^{r}$ & $\begin{array}{l}\text { Subcloned from pENTR4 rab-37.2 } \\
\text { Digested with BamH1/EcoR1 }\end{array}$ & Cloned in this study \\
\hline $\begin{array}{l}\text { pGST parallel } 2 \\
r a b-39\end{array}$ & $A m p^{r}$ & $\begin{array}{l}\text { Subcloned from pENTR4 rab-39 } \\
\text { Digested with BamH1/Xho1 }\end{array}$ & Cloned in this study \\
\hline $\begin{array}{l}\text { pGST parallel } 2 \\
r a b-y 1\end{array}$ & $A m p^{r}$ & $\begin{array}{l}\text { Subcloned from pGEMT } r a b-y 1 \\
\text { Digested with Nco1/EcoR1 }\end{array}$ & Cloned in this study \\
\hline
\end{tabular}




\begin{tabular}{|c|c|c|c|}
\hline $\begin{array}{l}\text { pGST parallel } 2 \\
\text { rab-y2 }\end{array}$ & $A m p^{r}$ & $\begin{array}{l}\text { Subcloned from pENTR4 rab-y2 } \\
\text { Digested with Nco1/Xho1 }\end{array}$ & Cloned in this study \\
\hline $\begin{array}{l}\text { pGST parallel } 2 \\
r a b-y 3\end{array}$ & $A m p^{r}$ & $\begin{array}{l}\text { Subcloned from pENTR4 rab-y3 } \\
\text { Digested wth BamH1/EcoR1 }\end{array}$ & Cloned in this study \\
\hline $\begin{array}{l}\text { pGST parallel } 2 \\
\text { rab-y6 }\end{array}$ & $A m p^{r}$ & $\begin{array}{l}\text { Subcloned from pGEMT rab-y6 } \\
\text { Digested with BamH1/EcoR1 }\end{array}$ & Cloned in this study \\
\hline $\begin{array}{l}\text { pGST parallel } 2 \\
\text { glo-1 }\end{array}$ & $A m p^{r}$ & $\begin{array}{l}\text { Subcloned from pGEMT glo-1 } \\
\text { Digested with Nco1/Xho1 }\end{array}$ & Cloned in this study \\
\hline $\begin{array}{l}\text { pGST parallel } 2 t b c- \\
4\end{array}$ & $A m p^{r}$ & $\begin{array}{l}\text { Subcloned from L4440 tbc-4 } \\
\text { Digested with Age1/Xho1 }\end{array}$ & Cloned in this study \\
\hline $\begin{array}{l}\text { pGST parallel } 2 \\
\text { tbc-4 } 78-383\end{array}$ & $A m p^{r}$ & $\begin{array}{l}\text { PCR with oGQ2092/oGQ2093 } \\
\text { Digested with Age1/Xho1 }\end{array}$ & Cloned in this study \\
\hline $\begin{array}{l}\text { Genes of interest } \\
\text { in Y2H vectors }\end{array}$ & $\begin{array}{l}\text { Resistance } \\
\text { Gene }\end{array}$ & Cloning Strategy & Source \\
\hline $\begin{array}{l}\text { pGBKT7 } r a b-1 \\
(Q 70 L) \\
\text { pGBKT7 } r a b-2 \\
(Q 65 L)\end{array}$ & $\begin{array}{l}\mathrm{Kan}^{\mathrm{r}} \\
\mathrm{Kan}^{\mathrm{r}}\end{array}$ & -- & $\begin{array}{l}\text { Kindly provided by } \\
\text { Katrin Schwarze } \\
\text { Kindly provided by } \\
\text { Marija Sumakovic }\end{array}$ \\
\hline $\begin{array}{l}\text { pGBKT7 } r a b-3 \\
(Q 81 L)\end{array}$ & $\operatorname{Kan}^{\mathrm{r}}$ & $\begin{array}{l}\text { Mutagenesis PCR with } \\
\text { oGQ1133/oGQ1134 } \\
\text { Digested with Nde1/Xho1 }\end{array}$ & Cloned in this study \\
\hline $\begin{array}{l}\text { pGBKT7 rab-5 } \\
(Q 78 L)\end{array}$ & $\operatorname{Kan}^{\mathrm{r}}$ & $\begin{array}{l}\text { Mutagenesis PCR with } \\
\text { oGQ675/oGQ676 } \\
\text { Digested with Nde1/EcoR1 }\end{array}$ & Cloned in this study \\
\hline $\begin{array}{l}\text { pGBKT7 rab-6.1 } \\
(Q 70 L)\end{array}$ & $\mathrm{Kan}^{\mathrm{r}}$ & -- & $\begin{array}{l}\text { Kindly provided by } \\
\text { Katrin Schwarze }\end{array}$ \\
\hline $\begin{array}{l}\text { pGBKT7 rab-6.2 } \\
(Q 69 L)\end{array}$ & $\operatorname{Kan}^{r}$ & -- & $\begin{array}{l}\text { Kindly provided by } \\
\text { Katrin Schwarze }\end{array}$ \\
\hline $\begin{array}{l}\text { pGBKT7 rab-7 } \\
(Q 68 L)\end{array}$ & $\operatorname{Kan}^{\mathrm{r}}$ & $\begin{array}{l}\text { Mutagenesis PCR with } \\
\text { oGQ997/oGQ998 } \\
\text { Digested with Nde1/Xho1 }\end{array}$ & Cloned in this study \\
\hline $\begin{array}{l}\text { pGBKT7 } r a b-8 \\
(Q 67 L)\end{array}$ & $\mathrm{Kan}^{\mathrm{r}}$ & $\begin{array}{l}\text { Mutagenesis PCR with } \\
\text { oGQ558/oGQ559 } \\
\text { Digested with Nde1/EcoR1 }\end{array}$ & Cloned in this study \\
\hline $\begin{array}{l}\text { pGBKT7 rab-10 } \\
(Q 68 L)\end{array}$ & $\operatorname{Kan}^{r}$ & $\begin{array}{l}\text { Mutagenesis PCR with } \\
\text { oGQ1137/oGQ1138Digested with } \\
\text { Nde1/EcoR1 }\end{array}$ & Cloned in this study \\
\hline $\begin{array}{l}\text { pGBKT7 rab-11.1 } \\
(Q 70 L)\end{array}$ & $\operatorname{Kan}^{\mathrm{r}}$ & $\begin{array}{l}\text { Mutagenesis PCR with } \\
\text { oGQ1141/oGQ1142 } \\
\text { Digested with Nco1/EcoR1 }\end{array}$ & Cloned in this study \\
\hline $\begin{array}{l}\text { pGBKT7 rab-14 } \\
(Q 70 L)\end{array}$ & $\operatorname{Kan}^{\mathrm{r}}$ & $\begin{array}{l}\text { Mutagenesis PCR with } \\
\text { oGQ246/oGQ247 } \\
\text { Digested with Nde1/Xho1 }\end{array}$ & Cloned in this study \\
\hline $\begin{array}{l}\text { pGBKT7 rab-18 } \\
(Q 70 L)\end{array}$ & $\operatorname{Kan}^{r}$ & $\begin{array}{l}\text { Mutagenesis PCR with } \\
\text { oGQ582/oGQ583 } \\
\text { Digested with Nde1/EcoR1 }\end{array}$ & Cloned in this study \\
\hline $\begin{array}{l}\text { pGBKT7 rab-19 } \\
(Q 69 L)\end{array}$ & $\mathrm{Kan}^{\mathrm{r}}$ & $\begin{array}{l}\text { Mutagenesis PCR with } \\
\text { oGQ566/oGQ567 } \\
\text { Digested with Nde1/EcoR1 }\end{array}$ & Cloned in this study \\
\hline $\begin{array}{l}\text { pGBKT7 rab-21 } \\
(Q 71 L)\end{array}$ & $\mathrm{Kan}^{\mathrm{r}}$ & $\begin{array}{l}\text { Mutagenesis PCR with } \\
\text { oGQ570/oGQ571 } \\
\text { Digested with Nde1/EcoR1 }\end{array}$ & Cloned in this study \\
\hline $\begin{array}{l}\text { pGBKT7 rab-27 } \\
(Q 75 L)\end{array}$ & $\operatorname{Kan}^{\mathrm{r}}$ & $\begin{array}{l}\text { Mutagenesis PCR with } \\
\text { oGQ1150/oGQ1151 } \\
\text { Digested with NcoI/Xho1 }\end{array}$ & Cloned in this study \\
\hline $\begin{array}{l}\text { pGBKT7 rab-28 } \\
(Q 95 L)\end{array}$ & $\operatorname{Kan}^{\mathrm{r}}$ & $\begin{array}{l}\text { Mutagenesis PCR with } \\
\text { oGQ574/oGQ575 } \\
\text { Digested with Nco1/EcoR1 }\end{array}$ & Cloned in this study \\
\hline
\end{tabular}




\begin{tabular}{|c|c|}
\hline $\begin{array}{l}\text { pGBKT7 rab-30 } \\
(Q 66 L)\end{array}$ & $\operatorname{Kan}^{r}$ \\
\hline $\begin{array}{l}\text { pGBKT7 rab-33 } \\
(Q 159 L)\end{array}$ & $\operatorname{Kan}^{\mathrm{r}}$ \\
\hline $\begin{array}{l}\text { pGBKT7 rab-35 } \\
(Q 69 L)\end{array}$ & $\operatorname{Kan}^{\mathrm{r}}$ \\
\hline $\begin{array}{l}\text { pGBKT7 } r a b-37.1 \\
(Q 37 L)\end{array}$ & $\operatorname{Kan}^{\mathrm{r}}$ \\
\hline $\begin{array}{l}\text { pGBKT7 } r a b-37.2 \\
\text { (Q89L) }\end{array}$ & $\operatorname{Kan}^{\mathrm{r}}$ \\
\hline $\begin{array}{l}\text { pGBKT7 rab-39 } \\
(Q 77 L)\end{array}$ & $\operatorname{Kan}^{\mathrm{r}}$ \\
\hline $\begin{array}{l}\text { pGBKT7 rab-y1 } \\
(Q 520 L)\end{array}$ & $\operatorname{Kan}^{r}$ \\
\hline $\begin{array}{l}\text { pGBKT7 rab-y2 } \\
(Q 262 L)\end{array}$ & $\operatorname{Kan}^{\mathrm{r}}$ \\
\hline $\begin{array}{l}\text { pGBKT7 rab-y3 } \\
(M 65 L)\end{array}$ & $\operatorname{Kan}^{\mathrm{r}}$ \\
\hline $\begin{array}{l}\text { pGBKT7 rab-y6 } \\
(Q 115 L)\end{array}$ & $\operatorname{Kan}^{\mathrm{r}}$ \\
\hline $\begin{array}{l}\text { pGBKT7 glo-1 } \\
(Q 71 L)\end{array}$ & $\operatorname{Kan}^{\mathrm{r}}$ \\
\hline $\begin{array}{l}\text { pGBKT7 rab-1 } \\
(S 25 N)\end{array}$ & $\operatorname{Kan}^{\mathrm{r}}$ \\
\hline $\begin{array}{l}\text { pGBKT7 } r a b-2 \\
(S 20 N)\end{array}$ & $\operatorname{Kan}^{\mathrm{r}}$ \\
\hline $\begin{array}{l}\text { pGBKT7 rab-3 } \\
(T 36 N)\end{array}$ & $\operatorname{Kan}^{\mathrm{r}}$ \\
\hline $\begin{array}{l}\text { pGBKT7 rab-5 } \\
(S 33 N)\end{array}$ & $\operatorname{Kan}^{\mathrm{r}}$ \\
\hline $\begin{array}{l}\text { pGBKT7 rab-6.1 } \\
(T 25 N)\end{array}$ & $\operatorname{Kan}^{\mathrm{r}}$ \\
\hline $\begin{array}{l}\text { pGBKT7 rab-6.2 } \\
(T 24 N)\end{array}$ & $\operatorname{Kan}^{\mathrm{r}}$ \\
\hline $\begin{array}{l}\text { pGBKT7 } r a b-7 \\
(T 23 N)\end{array}$ & $\operatorname{Kan}^{\mathrm{r}}$ \\
\hline $\begin{array}{l}\text { pGBKT7 } r a b-8 \\
(T 22 N)\end{array}$ & $\operatorname{Kan}^{r}$ \\
\hline $\begin{array}{l}\text { pGBKT7 } r a b-10 \\
(T 23 N)\end{array}$ & $\operatorname{Kan}^{\mathrm{r}}$ \\
\hline pGBKT7 rab-11.1 & \\
\hline
\end{tabular}

Mutagenesis PCR with

oGQ562/oGQ563

Digested with Nde1/Xho1

Mutagenesis PCR with

oGQ578/oGQ579

Digested with Nde1/EcoR1

Mutagenesis PCR with

oGQ1154/oGQ1155

Digested with Nde1/EcoR1

Mutagenesis PCR with

oGQ1158/oGQ1159

Digested with Nde1/EcoR1

Mutagenesis PCR with

oGQ1158/oGQ1159

Digested with Nde1/EcoR1

Mutagenesis PCR with

oGQ1162/oGQ1163

Digested with Nco1/Xho1

Mutagenesis PCR with

oGQ1347/oGQ1348

Digested with Nde1/EcoR1

Mutagenesis PCR with

oGQ1166/oGQ1167

Digested with Nco1/Xho1

Mutagenesis PCR with

oGQ1170/oGQ1171

Digested with Nde1/EcoR1

Mutagenesis PCR with

oGQ1174/oGQ1175

Digested with Nde1/EcoR1

Mutagenesis PCR with

oGQ1180/oGQ1181

Digested with Nco1/EcoR1

--

$--$

Mutagenesis PCR with

oGQ1131/oGQ1132

Digested with Nde1/Xho1

Mutagenesis PCR with

oGQ673/oGQ674

Digested with Nde1/EcoR1

$--$

Mutagenesis PCR with oGQ995/oGQ996

Digested with Nde1/Xho1

Mutagenesis PCR with

oGQ556/oGQ557

Digested with Nde1/EcoR1

Mutagenesis PCR with

oGQ1135/oGQ1136

Digested with Nde1/EcoR1

Mutagenesis PCR with

oGQ1139/oGQ1140Digested with

Nco1/EcoR1
Cloned in this study

Cloned in this study

Cloned in this study

Cloned in this study

Cloned in this study

Cloned in this study

Cloned in this study

Cloned in this study

Cloned in this study

Cloned in this study

Cloned in this study

Kindly provided by

Katrin Schwarze

Kindly provided by Marija Sumakovic

Cloned in this study

Cloned in this study

Kindly provided by Katrin Schwarze Kindly provided by Katrin Schwarze Cloned in this study

Cloned in this study

Cloned in this study

Cloned in this study 


\begin{tabular}{|c|c|c|c|}
\hline $\begin{array}{l}\text { pGBKT7 rab-14 } \\
(S 25 N)\end{array}$ & $\operatorname{Kan}^{\mathrm{r}}$ & $\begin{array}{l}\text { Mutagenesis PCR with } \\
\text { oGQ244/oGQ245 } \\
\text { Digested with Nde1/Xho1 }\end{array}$ & Cloned in this study \\
\hline $\begin{array}{l}\text { pGBKT7 } r a b-18 \\
(S 25 N)\end{array}$ & $\mathrm{Kan}^{\mathrm{r}}$ & $\begin{array}{l}\text { Mutagenesis PCR with } \\
\text { oGQ580/oGQ581 } \\
\text { Digested with Nde1/EcoR1 }\end{array}$ & Cloned in this study \\
\hline $\begin{array}{l}\text { pGBKT7 rab-19 } \\
(T 24 N)\end{array}$ & $\operatorname{Kan}^{\mathrm{r}}$ & $\begin{array}{l}\text { Mutagenesis PCR with } \\
\text { oGQ564/oGQ565 } \\
\text { Digested with Nde1/EcoR1 }\end{array}$ & Cloned in this study \\
\hline $\begin{array}{l}\text { pGBKT7 rab-21 } \\
(S 26 N)\end{array}$ & $\operatorname{Kan}^{\mathrm{r}}$ & $\begin{array}{l}\text { Mutagenesis PCR with } \\
\text { oGQ568/oGQ569 } \\
\text { Digested with Nde1/EcoR1 }\end{array}$ & Cloned in this study \\
\hline $\begin{array}{l}\text { pGBKT7 rab-27 } \\
(T 21 N)\end{array}$ & $\operatorname{Kan}^{r}$ & $\begin{array}{l}\text { Mutagenesis PCR with } \\
\text { oGQ1148/oGQ1149 } \\
\text { Digested with NcoI/Xho1 }\end{array}$ & Cloned in this study \\
\hline $\begin{array}{l}\text { pGBKT7 } r a b-28 \\
(T 49 N)\end{array}$ & $\operatorname{Kan}^{\mathrm{r}}$ & $\begin{array}{l}\text { Mutagenesis PCR with } \\
\text { oGQ572/oGQ573 } \\
\text { Digested with Nco1/EcoR1 }\end{array}$ & Cloned in this study \\
\hline $\begin{array}{l}\text { pGBKT7 rab-30 } \\
(T 21 N)\end{array}$ & $\operatorname{Kan}^{\mathrm{r}}$ & $\begin{array}{l}\text { Mutagenesis PCR with } \\
\text { oGQ560/oGQ561 } \\
\text { Digested with Nde1/Xho1 }\end{array}$ & Cloned in this study \\
\hline $\begin{array}{l}\text { pGBKT7 rab-33 } \\
(T 114 N)\end{array}$ & $\operatorname{Kan}^{r}$ & $\begin{array}{l}\text { Mutagenesis PCR with } \\
\text { oGQ576/oGQ577 } \\
\text { Digested with Nde1/EcoR1 }\end{array}$ & Cloned in this study \\
\hline $\begin{array}{l}\text { pGBKT7 rab-35 } \\
(S 24 N)\end{array}$ & $\operatorname{Kan}^{r}$ & $\begin{array}{l}\text { Mutagenesis PCR with } \\
\text { oGQ1152/oGQ1153 } \\
\text { Digested with Nde1/EcoR1 }\end{array}$ & Cloned in this study \\
\hline $\begin{array}{l}\text { pGBKT7 rab-37.1 } \\
(T 11 N)\end{array}$ & $\mathrm{Kan}^{\mathrm{r}}$ & $\begin{array}{l}\text { Mutagenesis PCR with } \\
\text { oGQ1156/oGQ1157 } \\
\text { Digested with Nde1/EcoR1 }\end{array}$ & Cloned in this study \\
\hline $\begin{array}{l}\text { pGBKT7 } r a b-37.2 \\
(T 43 N)\end{array}$ & $\operatorname{Kan}^{r}$ & $\begin{array}{l}\text { Mutagenesis PCR with } \\
\text { oGQ1156/oGQ1157 } \\
\text { Digested with Nde1/EcoR1 }\end{array}$ & Cloned in this study \\
\hline $\begin{array}{l}\text { pGBKT7 rab-39 } \\
(S 31 N)\end{array}$ & $\operatorname{Kan}^{r}$ & $\begin{array}{l}\text { Mutagenesis PCR with } \\
\text { oGQ1160/oGQ1161 } \\
\text { Digested with Nco1/Xho1 }\end{array}$ & Cloned in this study \\
\hline $\begin{array}{l}\text { pGBKT7 rab-y1 } \\
(S 475 N)\end{array}$ & $\mathrm{Kan}^{\mathrm{r}}$ & $\begin{array}{l}\text { Mutagenesis PCR with } \\
\text { oGQ1345/oGQ1346 } \\
\text { Digested with Nde1/EcoR1 }\end{array}$ & Cloned in this study \\
\hline $\begin{array}{l}\text { pGBKT7 } r a b-y 2 \\
(T 216 N)\end{array}$ & $\mathrm{Kan}^{\mathrm{r}}$ & $\begin{array}{l}\text { Mutagenesis PCR with } \\
\text { oGQ1164/oGQ1165 } \\
\text { Digested with Nco1/Xho1 }\end{array}$ & Cloned in this study \\
\hline $\begin{array}{l}\text { pGBKT7 rab-y3 } \\
(S 20 N)\end{array}$ & $\operatorname{Kan}^{\mathrm{r}}$ & $\begin{array}{l}\text { Mutagenesis PCR with } \\
\text { oGQ1168/oGQ1169 } \\
\text { Digested with Nde1/EcoR1 }\end{array}$ & Cloned in this study \\
\hline $\begin{array}{l}\text { pGBKT7 rab-y6 } \\
(T 66 N)\end{array}$ & $\operatorname{Kan}^{r}$ & $\begin{array}{l}\text { Mutagenesis PCR with } \\
\text { oGQ1172/oGQ1173 } \\
\text { Digested with Nde1/EcoR1 }\end{array}$ & Cloned in this study \\
\hline $\begin{array}{l}\text { pGBKT7 glo-1 } \\
(T 25 N)\end{array}$ & $\mathrm{Kan}^{\mathrm{r}}$ & $\begin{array}{l}\text { Mutagenesis PCR with } \\
\text { oGQ1178/oGQ1179 } \\
\text { Digested with Nco1/EcoR1 }\end{array}$ & Cloned in this study \\
\hline pGBKT7 rabn-5 & $\operatorname{Kan}^{r}$ & $\begin{array}{l}\text { Subcloned from L4440 rabn-5 } \\
\text { Digested with Age1/EcoR1 }\end{array}$ & Cloned in this study \\
\hline pGADT7 tbc-4 & $A m p^{r}$ & $\begin{array}{l}\text { Subcloned from L } 4440 t b c-4 \\
\text { Digested with Age } 1 / \text { Xho } 1\end{array}$ & Cloned in this study \\
\hline $\begin{array}{l}\text { pGADT7 tbc-4 } \\
R 155 A\end{array}$ & $A m p^{r}$ & $\begin{array}{l}\text { Mutagenesis PCR with } \\
\text { oGQ1700/oGQ1701 } \\
\text { Digested with Age1/Xho1 }\end{array}$ & Cloned in this study \\
\hline $\begin{array}{l}\text { pGADT7 tbc-4 1- } \\
100\end{array}$ & $A m p^{r}$ & $\begin{array}{l}\text { PCR with oGQ1590/oGQ2198 } \\
\text { Digested with Age1/Xho1 }\end{array}$ & Cloned in this study \\
\hline
\end{tabular}




\begin{tabular}{|c|c|c|c|}
\hline pGADT7 tbc-4 78- & $A m p^{r}$ & PCR with oGQ2092/oGQ1697 & Cloned in this study \\
\hline $\begin{array}{l}825 \\
R 155 A\end{array}$ & & Digested with Age1/Xho1 & \\
\hline $\begin{array}{l}\text { pGADT7 tbc-4 377- } \\
825\end{array}$ & $A m p^{r}$ & $\begin{array}{l}\text { PCR with oGQ1601/oGQ1602 } \\
\text { Digested with Age1/Xho1 }\end{array}$ & Cloned in this study \\
\hline pGADT7 ehbp-1 & $A m p^{r}$ & $\begin{array}{l}\text { PCR with oGQ1583/oGQ1584 } \\
\text { Digested with Age1/Xho1 }\end{array}$ & Cloned in this study \\
\hline $\begin{array}{l}\text { pGADT7 ehbp-1 } \\
662-901\end{array}$ & $A m p^{r}$ & $\begin{array}{l}\text { PCR with oGQ1715/oGQ1584 } \\
\text { Digested with Age1/Xho1 }\end{array}$ & Cloned in this study \\
\hline $\begin{array}{l}\text { Genes of interest } \\
\text { in injection vectors }\end{array}$ & $\begin{array}{l}\text { Resistance } \\
\text { Gene }\end{array}$ & Cloning strategy & Source \\
\hline $\begin{array}{l}\text { pPD115.62 prab- } \\
2: \because g f p\end{array}$ & $\mathrm{Amp}^{\mathrm{r}}$ & -- & $\begin{array}{l}\text { Kindly provided by } \\
\text { Marija Sumakovic }\end{array}$ \\
\hline $\begin{array}{l}\text { pPD1 } 15.62 \text { prab- } \\
3: \because g f p\end{array}$ & $\mathrm{Amp}^{\mathrm{r}}$ & -- & $\begin{array}{l}\text { Kindly provided by } \\
\text { Marija Sumakovic }\end{array}$ \\
\hline $\begin{array}{l}\text { pPD115.62 prab- } \\
5: \because g f p\end{array}$ & $\mathrm{Amp}^{\mathrm{r}}$ & $\begin{array}{l}\text { PCR with oGQ730/oGQ731 } \\
\text { Digested with Hind3/Kpn1 }\end{array}$ & Cloned in this study \\
\hline $\begin{array}{l}\text { pPD115.62 prab- } \\
6.1: \because g f p\end{array}$ & $\mathrm{Amp}^{\mathrm{r}}$ & $\begin{array}{l}\text { PCR with oGQ732/oGQ733 } \\
\text { Digested with Hind3/Age1 }\end{array}$ & Cloned in this study \\
\hline $\begin{array}{l}\text { pPD115.62 prab- } \\
6.2: \because g f p\end{array}$ & $A m p^{r}$ & $\begin{array}{l}\text { PCR with oGQ734/oGQ735 } \\
\text { Digested with Hind3/Age1 }\end{array}$ & Cloned in this study \\
\hline $\begin{array}{l}\text { pPD115.62 prab- } \\
7: \because g f p\end{array}$ & $\mathrm{Amp}^{\mathrm{r}}$ & $\begin{array}{l}\text { PCR with oGQ736/oGQ737 } \\
\text { Digested with Hind3/Age1 }\end{array}$ & Cloned in this study \\
\hline $\begin{array}{l}\text { pPD115.62 prab- } \\
8: \because g f p\end{array}$ & $A m p^{r}$ & $\begin{array}{l}\text { PCR with oGQ484/oGQ485 } \\
\text { Digested with Hind3/Age1 }\end{array}$ & Cloned in this study \\
\hline $\begin{array}{l}\text { pPD115.62 prab- } \\
7: \because g f p\end{array}$ & $A m p^{r}$ & $\begin{array}{l}\text { PCR with oGQ736/oGQ737 } \\
\text { Digested with Hind3/Age1 }\end{array}$ & Cloned in this study \\
\hline $\begin{array}{l}\text { pPD115.62 prab- } \\
8: \because g f p\end{array}$ & $A m p^{r}$ & $\begin{array}{l}\text { PCR with oGQ484/oGQ485 } \\
\text { Digested with Hind3/Age1 }\end{array}$ & Cloned in this study \\
\hline $\begin{array}{l}\text { pPD115.62 prab- } \\
10: \because g f p\end{array}$ & $A m p{ }^{r}$ & $\begin{array}{l}\text { PCR with oGQ738/oGQ739 } \\
\text { Digested with Hind3/Age1 }\end{array}$ & Cloned in this study \\
\hline $\begin{array}{l}\text { pPD115.62 prab- } \\
11.1: \because g f p\end{array}$ & $A m p^{r}$ & $\begin{array}{l}\text { PCR with oGQ740/oGQ741 } \\
\text { Digesed with Pst1/Kpn1 }\end{array}$ & Cloned in this study \\
\hline $\begin{array}{l}\text { pPD115.62 prab- } \\
11.2:: g f p\end{array}$ & $A m p^{r}$ & $\begin{array}{l}\text { PCR with oGQ742/oGQ743 } \\
\text { Digested with Pst1/Age1 }\end{array}$ & \\
\hline $\begin{array}{l}\text { pPD115.62 prab- } \\
14: \because g f p\end{array}$ & $A m p^{r}$ & -- & $\begin{array}{l}\text { Kindly provided by } \\
\text { Stefan Eimer }\end{array}$ \\
\hline $\begin{array}{l}\text { pPD115.62 prab- } \\
18.1:: g f p\end{array}$ & $A m p^{r}$ & $\begin{array}{l}\text { PCR with oGQ490/oGQ491 } \\
\text { Digested wth Hind3/Kpn1 }\end{array}$ & Cloned in this study \\
\hline $\begin{array}{l}\text { pPD115.62 prab- } \\
18.2: \because g f p\end{array}$ & $A m p^{r}$ & $\begin{array}{l}\text { PCR with oGQ492/oGQ493 } \\
\text { Digested wth Hind3/Kpn1 }\end{array}$ & Cloned in this study \\
\hline $\begin{array}{l}\text { pPD115.62 prab- } \\
19:: g f p\end{array}$ & $A m p^{r}$ & $\begin{array}{l}\text { PCR with oGQ488/oGQ489 } \\
\text { Digested with Pst1/Kpn1 }\end{array}$ & Cloned in this study \\
\hline $\begin{array}{l}\text { pPD115.62 prab- } \\
21: \because g f p\end{array}$ & $A m p^{r}$ & $\begin{array}{l}\text { PCR with oGQ494/oGQ495 } \\
\text { Digested with Hind3/Kpn1 }\end{array}$ & Cloned in this study \\
\hline $\begin{array}{l}\text { pPD115.62 prab- } \\
27: \because g f p\end{array}$ & $A m p^{r}$ & $\begin{array}{l}\text { PCR with oGQ744/oGQ745 } \\
\text { Digested with Pst1/Age1 }\end{array}$ & Cloned in this study \\
\hline $\begin{array}{l}\text { pPD115.62 prab- } \\
28: \because g f p\end{array}$ & $A m p^{r}$ & $\begin{array}{l}\text { PCR with oGQ498/oGQ499 } \\
\text { Digested with Pst1/Age1 }\end{array}$ & Cloned in this study \\
\hline $\begin{array}{l}\text { pPD115.62 prab- } \\
30: \because g f p\end{array}$ & $A m p^{r}$ & $\begin{array}{l}\text { PCR with oGQ486/oGQ487 } \\
\text { Digested with Hind3/Kpn1 }\end{array}$ & Cloned in this study \\
\hline $\begin{array}{l}\text { pPD115.62 prab- } \\
33: \because g f p\end{array}$ & $A m p^{r}$ & $\begin{array}{l}\text { PCR with oGQ496/oGQ497 } \\
\text { Digested with Hind3/Kpn1 }\end{array}$ & Cloned in this study \\
\hline $\begin{array}{l}\text { pPD115.62 prab- } \\
35: \because g f p\end{array}$ & $A m p^{r}$ & $\begin{array}{l}\text { PCR with oGQ684/oGQ685 } \\
\text { Digested with Pst1/Kpn1 }\end{array}$ & Cloned in this study \\
\hline
\end{tabular}




\begin{tabular}{|c|c|c|c|}
\hline $\begin{array}{l}\text { pPD115.62 prab- } \\
37.1:: g f p\end{array}$ & $A m p^{r}$ & $\begin{array}{l}\text { PCR with oGQ866/oGQ867 } \\
\text { Digested with Pst1/Age1 }\end{array}$ & Cloned in this study \\
\hline $\begin{array}{l}\text { pPD115.62 prab- } \\
37.2: \because g f p\end{array}$ & $A m p^{r}$ & $\begin{array}{l}\text { PCR with oGQ866/oGQ869 } \\
\text { Digested with Pst1/Age1 }\end{array}$ & Cloned in this study \\
\hline $\begin{array}{l}\text { pPD115.62 prab- } \\
39: \because g f p\end{array}$ & $A m p^{r}$ & $\begin{array}{l}\text { PCR with oGQ677/oGQ678 } \\
\text { Digested with Hind3/Age1 }\end{array}$ & Cloned in this study \\
\hline $\begin{array}{l}\text { pPD115.62 prab- } \\
y 2: \because g f p\end{array}$ & $A m p^{r}$ & $\begin{array}{l}\text { PCR with oGQ621/oGQ622 } \\
\text { Digested with Pst1/Kpn1 }\end{array}$ & Cloned in this study \\
\hline $\begin{array}{l}\text { pPD115.62 prab- } \\
y 3: \because g f p\end{array}$ & $A m p^{r}$ & $\begin{array}{l}\text { PCR with oGQ623/oGQ624 } \\
\text { Digested with Pst1/Kpn1 }\end{array}$ & Cloned in this study \\
\hline pGEMT prab-y4 & $A m p^{r}$ & $\begin{array}{l}\text { PCR with oGQ625/oGQ626 } \\
\text { TA cloning }\end{array}$ & Cloned in this study \\
\hline $\begin{array}{l}\text { pPD115.62 prab- } \\
y 4:: g f p\end{array}$ & $A m p^{r}$ & $\begin{array}{l}\text { subcloned from pPD115.62/pGEMT } \\
\text { prab-y4 } \\
\text { Digested with Kpn1/Spe1 }\end{array}$ & Cloned in this study \\
\hline $\begin{array}{l}\text { pPD115.62 prab- } \\
\text { y5::gfp }\end{array}$ & $A m p^{r}$ & $\begin{array}{l}\text { PCR with oGQ627/oGQ628Digested } \\
\text { with Hind3/Age1 }\end{array}$ & Cloned in this study \\
\hline $\begin{array}{l}\text { pPD115.62 prab- } \\
y 6: \because g f p\end{array}$ & $A m p^{r}$ & $\begin{array}{l}\text { PCR with oGQ679/oGQ680 } \\
\text { Digested with Pst1/Age1 }\end{array}$ & Cloned in this study \\
\hline $\begin{array}{l}\text { pPD1 } 15.62 \text { glo- } \\
1: g f p\end{array}$ & $A m p^{r}$ & $\begin{array}{l}\text { PCR with oGQ943/oGQ944 } \\
\text { Digested with Hind3/Age1 }\end{array}$ & Cloned in this study \\
\hline $\begin{array}{l}\text { prab-3::tagRFP- } \\
\text { rab-1 }\end{array}$ & $A m p^{r}$ & -- & $\begin{array}{l}\text { Kindly provided by } \\
\text { Mandy Hannemann }\end{array}$ \\
\hline $\begin{array}{l}\text { prab-3::mcherry- } \\
\text { rab2 }\end{array}$ & $A m p^{r}$ & -- & $\begin{array}{l}\text { Kindly provided by } \\
\text { Marija Sumakovic }\end{array}$ \\
\hline $\begin{array}{l}\text { prab-3::mcherry- } \\
\text { rab3 }\end{array}$ & $A m p^{r}$ & -- & $\begin{array}{l}\text { Kindly provided by } \\
\text { Marija Sumakovic }\end{array}$ \\
\hline $\begin{array}{l}\text { prab-3::mcherry- } \\
\text { rab-5 }\end{array}$ & $A m p^{r}$ & -- & $\begin{array}{l}\text { Kindly provided by } \\
\text { Marija Sumakovic }\end{array}$ \\
\hline $\begin{array}{l}\text { prab-3::mcherry- } \\
\text { rab-6.1 }\end{array}$ & $A m p^{r}$ & -- & $\begin{array}{l}\text { Kindly provided by } \\
\text { Marija Sumakovic }\end{array}$ \\
\hline $\begin{array}{l}\text { prab-3::mcherry- } \\
\text { rab- } 6.2\end{array}$ & $A m p^{r}$ & -- & $\begin{array}{l}\text { Kindly provided by } \\
\text { Marija Sumakovic }\end{array}$ \\
\hline $\begin{array}{l}\text { prab-3::mcherry- } \\
\text { rab-7 }\end{array}$ & $A m p^{r}$ & -- & $\begin{array}{l}\text { Kindly provided by } \\
\text { Marija Sumakovic }\end{array}$ \\
\hline $\begin{array}{l}\text { prab-3::tagRFP- } \\
\text { rab-8 }\end{array}$ & $A m p^{r}$ & $\begin{array}{l}\text { Subcloned from pENTR4 rab-8 } \\
\text { Digested with Age1/Xho1 }\end{array}$ & Cloned in this study \\
\hline $\begin{array}{l}\text { prab-3::mcherry- } \\
\text { rab-10 }\end{array}$ & $A m p^{r}$ & $\begin{array}{l}\text { Subcloned from pETNR4 rab-10 } \\
\text { Digested with Age1/Xho1 }\end{array}$ & Cloned in this study \\
\hline $\begin{array}{l}\text { prab-3::tagRFP- } \\
\text { rab-11.1 }\end{array}$ & $A m p^{r}$ & $\begin{array}{l}\text { Subcloned from pGEMT rab-11.1 } \\
\text { Digested with Age1/Xho1 }\end{array}$ & Cloned in this study \\
\hline $\begin{array}{l}\text { prab-3::mcherry- } \\
\text { rab-14 }\end{array}$ & $A m p^{r}$ & $\begin{array}{l}\text { Subcloned from pBluescript } r a b-14 \\
\text { Digested with Age1/Xhol }\end{array}$ & Cloned in this study \\
\hline $\begin{array}{l}\text { prab-3::tagRFP- } \\
\text { rab-18 }\end{array}$ & $A m p^{r}$ & $\begin{array}{l}\text { Subcloned from pENTR4 rab-18 } \\
\text { Digested with Age1/Xho1 }\end{array}$ & Cloned in this study \\
\hline $\begin{array}{l}\text { phsp- } \\
16.41:: \operatorname{tag} R F P-r a b- \\
19\end{array}$ & $A m p^{r}$ & $\begin{array}{l}\text { Subcloned from pENTR4 rab-19 } \\
\text { Digested with Age1/EcoR1 }\end{array}$ & Cloned in this study \\
\hline $\begin{array}{l}\text { prab-3::tagRFP- } \\
r a b-19\end{array}$ & $A m p^{r}$ & $\begin{array}{l}\text { Subcloned from phsp-16.41::tagRFP- } \\
\text { rab-19 Pst1/Kpn1 }\end{array}$ & Cloned in this study \\
\hline $\begin{array}{l}\text { prab-3::tagRFP- } \\
r a b-27\end{array}$ & $A m p^{r}$ & $\begin{array}{l}\text { Subcloned from pENTR4 rab-27 } \\
\text { Digested with Age1/Xho1 }\end{array}$ & Cloned in this study \\
\hline $\begin{array}{l}\text { prab-28::mcherry- } \\
\text { rab-28 }\end{array}$ & $A m p^{r}$ & $\begin{array}{l}\text { Subcloned 3-fragment ligation } \\
\text { prab28::gfp, mcherry, pBluescript rab- } \\
\text { 28. Digested with Kpn1/Not1/Xho1 }\end{array}$ & Cloned in this study \\
\hline
\end{tabular}




\begin{tabular}{|c|c|c|c|}
\hline $\begin{array}{l}\text { prab-30::mcherry- } \\
\text { rab-30 }\end{array}$ & $A m p^{r}$ & $\begin{array}{l}\text { Subcloned 3-fragment ligation } \\
\text { prab30::gfp, mcherry, pBluescript } r a b \text { - } \\
\text { 30. Digested with Kpn1/Not1/Xho1 }\end{array}$ & Cloned in this study \\
\hline $\begin{array}{l}\text { prab-3::tagRFP- } \\
\text { rab-33 }\end{array}$ & $A m p^{r}$ & $\begin{array}{l}\text { Subcloned from pENTR4 rab-33 } \\
\text { Digested with Age1/Xho1 }\end{array}$ & Cloned in this study \\
\hline $\begin{array}{l}\text { prab-3::tagRFP- } \\
\text { rab-35 }\end{array}$ & $A m p^{r}$ & $\begin{array}{l}\text { Subcloned from pENTR4 rab-35 } \\
\text { Digested with Age1/Xho1 }\end{array}$ & Cloned in this study \\
\hline $\begin{array}{l}\text { prab-3::tagRFP- } \\
\text { rab-37.1 }\end{array}$ & $A m p^{r}$ & $\begin{array}{l}\text { Subcloned from pENTR4 rab-37.1 } \\
\text { Digested with Age1/Xho1 }\end{array}$ & Cloned in this study \\
\hline $\begin{array}{l}\text { prab-3::tagRFP- } \\
r a b-37.2\end{array}$ & $A m p^{r}$ & $\begin{array}{l}\text { Subcloned from pENTR4 rab-37.2 } \\
\text { Digested with Age1/Xho1 }\end{array}$ & Cloned in this study \\
\hline $\begin{array}{l}\text { prab-3::tagRFP- } \\
r a b-39\end{array}$ & $A m p^{r}$ & $\begin{array}{l}\text { Subcloned from pENTR4 rab-39 } \\
\text { Digested with Age1/Xho1 }\end{array}$ & Cloned in this study \\
\hline $\begin{array}{l}\text { prab-3::tagRFP- } \\
\text { rab-y1 }\end{array}$ & $A m p^{r}$ & $\begin{array}{l}\text { Subcloned from pENTR4 rab-yl } \\
\text { Digested with Age1/Xho1 }\end{array}$ & Cloned in this study \\
\hline $\begin{array}{l}\text { prab-3::tagRFP- } \\
r a b-y 2\end{array}$ & $A m p^{r}$ & $\begin{array}{l}\text { Subcloned from pENTR4 } r a b \text { - } \\
\text { y2Digested with Age1/Xho1 }\end{array}$ & Cloned in this study \\
\hline $\begin{array}{l}\text { prab-3::tagRFP- } \\
\text { rab-y3 }\end{array}$ & $A m p^{r}$ & $\begin{array}{l}\text { Subcloned from pENTR4 rab-y3 } \\
\text { Digested with Age1/Xho1 }\end{array}$ & Cloned in this study \\
\hline $\begin{array}{l}\text { prab-3::tagRFP- } \\
\text { glo-1 }\end{array}$ & $A m p^{r}$ & $\begin{array}{l}\text { Subcloned from pGEMT glo-1 } \\
\text { Digested wit Age1/Xhol }\end{array}$ & Cloned in this study \\
\hline prab-3::cb5-gfp & $A m p^{r}$ & -- & $\begin{array}{l}\text { Kindly provided by } \\
\text { Marija Sumakovic }\end{array}$ \\
\hline prab-3::gfp-єcop & $A m p^{r}$ & -- & $\begin{array}{l}\text { Kindly provided by } \\
\text { Marija Sumakovic }\end{array}$ \\
\hline prab-3::mannsII-yfp & $A m p^{r}$ & -- & $\begin{array}{l}\text { Kindly provided by } \\
\text { Marija Sumakovic }\end{array}$ \\
\hline prab-3::gfp-2xfyve & $A m p^{r}$ & -- & $\begin{array}{l}\text { Kindly provided by } \\
\text { Marija Sumakovic }\end{array}$ \\
\hline $\begin{array}{l}\text { prab-3::tagRFP- } \\
\text { syx-6 }\end{array}$ & $A m p^{r}$ & -- & $\begin{array}{l}\text { Kindly provided by } \\
\text { Marija Sumakovic }\end{array}$ \\
\hline prab-3::apt-9-yfp & $A m p^{r}$ & -- & $\begin{array}{l}\text { Kindly provided by } \\
\text { Mandy Hannemann }\end{array}$ \\
\hline $\begin{array}{l}\text { prab-3::mcherry- } \\
\text { rab-5 (Q78L) }\end{array}$ & $A m p^{r}$ & -- & $\begin{array}{l}\text { Kindly provided by } \\
\text { Marija Sumakovic }\end{array}$ \\
\hline $\begin{array}{l}\text { prab-3::tagRFP- } \\
\text { tbc-4 }\end{array}$ & $A m p^{r}$ & $\begin{array}{l}\text { Subcloned from L4440 tbc-4 } \\
\text { Digested with Age1/Xho1 }\end{array}$ & Cloned in this study \\
\hline prab-3::gfp-tbc-4 & $A m p^{r}$ & $\begin{array}{l}\text { Subcloned from L4440 tbc-4 } \\
\text { Digested with Age } 1 / \text { Xho1 }\end{array}$ & Cloned in this study \\
\hline $\begin{array}{l}\text { prab-3::tagRFP- } \\
\text { tbc-4 (R155A) }\end{array}$ & $A m p^{r}$ & $\begin{array}{l}\text { Subcloned from pGADT7 } t b c-4 \\
(R 155 A)\end{array}$ & Cloned in this study \\
\hline $\begin{array}{l}\text { prab-3::ehbp-1- } \\
\operatorname{tagRFP}\end{array}$ & $A m p^{r}$ & $\begin{array}{l}\text { Digested with Age } 1 / \text { Xho } 1 \\
\text { Subcloned from L } 4440 \text { ehbp-1 } \\
\text { Digeested with Age1/Not1 }\end{array}$ & Cloned in this study \\
\hline prab-3::gfp-rab-10 & $A m p^{r}$ & $\begin{array}{l}\text { Subcloned from prab-3::mcherry-rab- } \\
\text { 10. Digested with Age } 1 / \text { Xho } 1\end{array}$ & Cloned in this study \\
\hline $\begin{array}{l}\text { prunc- } \\
129:: \text { mcherry-rab- } \\
10\end{array}$ & $A m p^{r}$ & $\begin{array}{l}\text { Subcloned from prab-3::mcherry-rab- } \\
10 \\
\text { Digested with Spe } 1 / \mathrm{Kpn} 1\end{array}$ & Cloned in this study \\
\hline prab-3::ctns-1-yfp & $A m p^{r}$ & -- & $\begin{array}{l}\text { Kindly provided by } \\
\text { Sabine Koenig }\end{array}$ \\
\hline $\begin{array}{l}\text { prab-3::mcherry- } \\
\text { rabn-5 }\end{array}$ & $A m p^{r}$ & $\begin{array}{l}\text { Subcloned from L4440 rabn-5 } \\
\text { Digested with Age1/EcoR1 }\end{array}$ & Cloned in this study \\
\hline
\end{tabular}




\subsection{C.elegans strains}

All mutant and transgenic strains used in this study are listed in table XII.2.

Table XII.2. List of all strains used in this study

\begin{tabular}{|c|c|}
\hline Strain name & Genotype \\
\hline Bristol & N2 (wild type) \\
\hline MT1093 & $r a b-2(n 501)$ \\
\hline GQ573 & $r a b-2(n u 415)$ \\
\hline NM791 & $r a b-3(j s 49)$ \\
\hline GQ640 & $r a b-6.1(\operatorname{tm} 2124)$ \\
\hline VC2117 & $r a b-6.2(o k 2254)$ \\
\hline VC308 & $r a b-7(o k 511)$ \\
\hline GQ574 & $r a b-8(\operatorname{tm} 2991)$ \\
\hline VC1026 & $r a b-10(o k 1494)$ \\
\hline GQ575 & $r a b-11.2(\operatorname{tm} 2081)$ \\
\hline GQ576 & $r a b-14(\operatorname{tm} 2095)$ \\
\hline RB1638 & $r a b-18(o k 2020)$ \\
\hline RB1537 & $r a b-19(o k 1845)$ \\
\hline VC1372 & $r a b-21(o k 1879)$ \\
\hline GQ577 & $r a b-27(\operatorname{tm} 2306)$ \\
\hline $\mathrm{RB} 2484$ & $r a b-28(o k 3424)$ \\
\hline RB1376 & $r a b-33(o k 1561)$ \\
\hline GQ578 & $r a b-35(\operatorname{tm} 2058)$ \\
\hline GQ579 & $r a b-37(\operatorname{tm} 2089)$ \\
\hline GQ580 & $r a b-39(\operatorname{tm} 2466)$ \\
\hline VC830 & $r a b-y 1(o k 1356)$ \\
\hline RB1553 & $r a b-y 3(o k 1871)$ \\
\hline JJ1271 & glo-1(zu391) \\
\hline DA509 & unc-31(e928) \\
\hline GQ581 & $t b c-2(q x 20)$ \\
\hline GQ582 & $t b c-2(t m 2241)$ \\
\hline VC2256 & $t b c-4(o k 2928)$ \\
\hline GQ583 & $t b c-4(t m 3255)$ \\
\hline RB1370 & rabn-5(ok1555) \\
\hline GR1373 & eri-1(mg366) \\
\hline WM49 & rde-4(ne301) \\
\hline SP1713 & $d y f-11(m n 392)$ \\
\hline GQ584 & nuIs 183 [punc129::nlp-21-venus] \\
\hline KP3292 & nuIs 152[punc-129::gfp-snb-1] \\
\hline KP3931 & nuIs 168[punc-129::venus-rab-3], \\
\hline GQ585 & nuIs195[punc-129::ins-22-venus] \\
\hline GQ586 & ceIs72[punc-129::ida-1-gfp] \\
\hline GQ587 & zxIs5[unc-17::chop-2(H134R)-yfp;lin-15(+)] \\
\hline GQ588 & zxIs6[unc-17::chop-2(H134R)-yfp;lin-15(+)] \\
\hline DH1336 & bIs34[prme-8::rme-8-gfp] \\
\hline GQ153 & gz110[prab-3::mcherry-rab-5Q78L]; nuIs183 [punc-129::nlp-21-venus] \\
\hline GQ026 & unc-108(n501); nuIs183 [punc-129::nlp-21-venus] \\
\hline GQ445 & rab-3(js49); nuIs183 [punc-129::nlp-21-venus] \\
\hline GQ446 & rab-6.2(ok2254); nuIs183 [punc-129::nlp-21-venus] \\
\hline GQ447 & rab-8(tm2991); nuIs183 [punc-129::nlp-21-venus] \\
\hline GQ448 & rab-10(ok1494); nuIs183 [punc-129::nlp-21-venus] \\
\hline GQ449 & rab-11.2(tm2081); nuIs183 [punc-129::nlp-21-venus] \\
\hline GQ450 & rab-14(tm2095); nuIs183 [punc-129::nlp-21-venus] \\
\hline GQ451 & rab-18(ok2020); nuIs183 [punc-129::nlp-21-venus] \\
\hline GQ452 & rab-19(ok1845); nuIs183 [punc-129::nlp-21-venus] \\
\hline GQ453 & rab-21(ok1879); nuIs183 [punc-129::nlp-21-venus] \\
\hline GQ454 & rab-27(tm2306); nuIs183 [punc-129::nlp-21-venus] \\
\hline
\end{tabular}




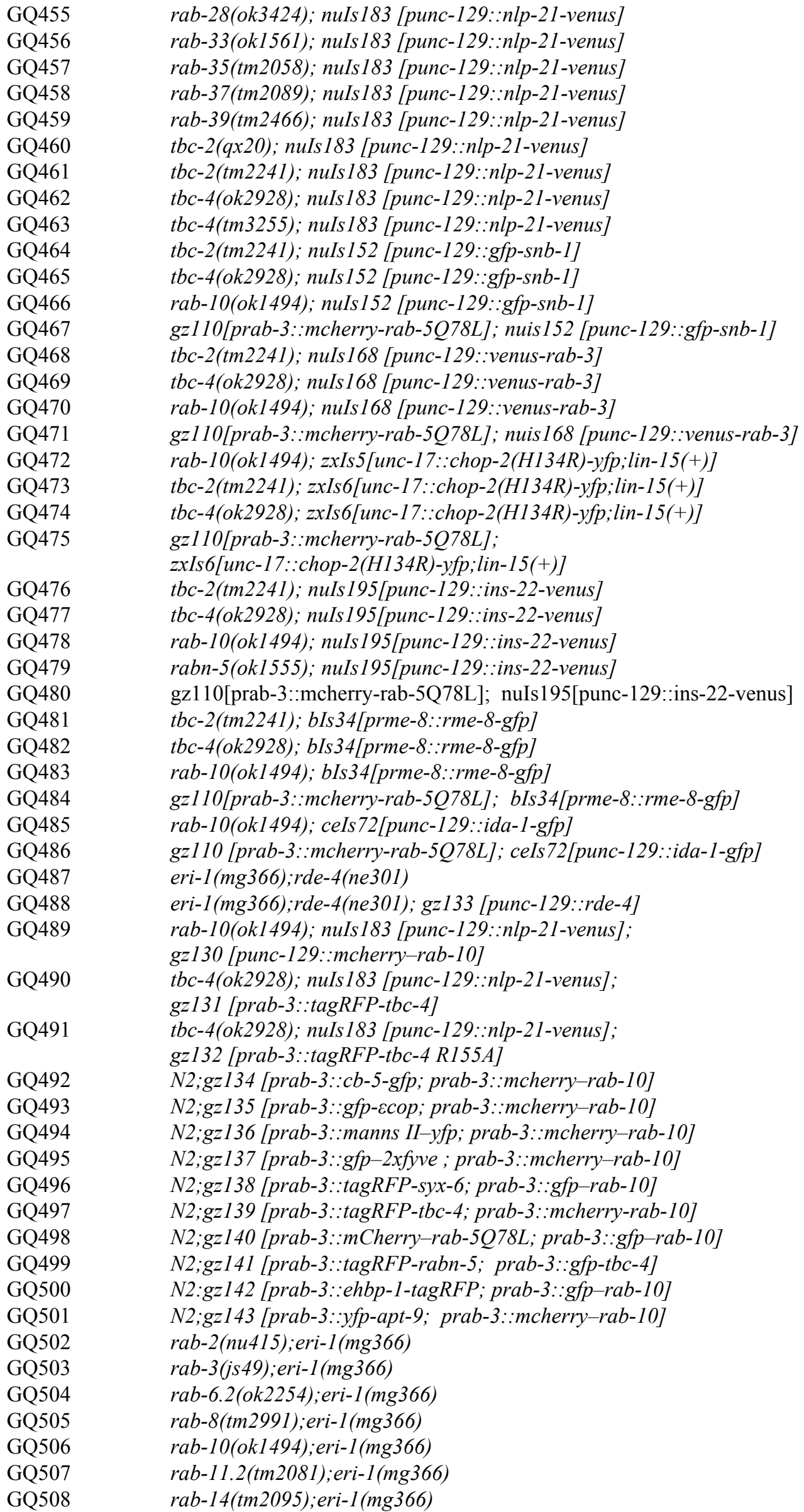




\begin{tabular}{|c|c|}
\hline GQ509 & rab-18(ok2020);eri-1(mg366) \\
\hline GQ510 & rab-19(ok1845);eri-1(mg366) \\
\hline GQ511 & rab-21(ok1879);eri-1(mg366) \\
\hline GQ512 & rab-27(tm2306);eri-1(mg366) \\
\hline GQ513 & rab-28(ok3424);eri-1(mg366) \\
\hline GQ514 & rab-33(ok1561);eri-1(mg366) \\
\hline GQ515 & rab-35(tm2058);eri-1(mg366) \\
\hline GQ516 & rab-37(tm2089);eri-1(mg366) \\
\hline GQ517 & rab-39(tm2466);eri-1(mg366) \\
\hline GQ518 & rab-y1(ok1356);eri-1(mg366) \\
\hline GQ519 & rab-y3(ok1871);eri-1(mg366) \\
\hline GQ520 & glo-1(zu391);eri-1(mg366) \\
\hline GQ521 & $r a b-3(j s 49) ; r a b-27(t m 2306) ; e r i-1(m g 366)$ \\
\hline GQ522 & $N 2 ; g z 144[p r a b-8:: g f p]$ \\
\hline GQ523 & $N 2 ; g z 145[p r a b-28: \because g f p]$ \\
\hline GQ524 & $N 2 ; g z 146[p r a b-30: \because g f p]$ \\
\hline GQ525 & $N 2 ; g z 147[p r a b-19: \because g f p]$ \\
\hline GQ526 & $N 2 ; g z 148[p r a b-18.1: \because g f p]$ \\
\hline GQ527 & $N 2 ; g z 149[p r a b-18.2: \because g f p]$ \\
\hline GQ528 & $N 2 ; g z 150[p r a b-21: \because g f p]$ \\
\hline GQ529 & $N 2 ; g z 151[p r a b-33: \because g f p]$ \\
\hline GQ530 & $N 2 ; g z 152[p r a b-y 2: \because g f p]$ \\
\hline GQ531 & N2;gz153[prab-y3::gfp] \\
\hline GQ532 & N2;gz154[prab-y5::gfp] \\
\hline GQ533 & N2;gz155[prab-39::gfp] \\
\hline GQ534 & N2;gz156[prab-y6::gfp] \\
\hline GQ535 & $N 2 ; g z 157[p r a b-y 4: \because g f p]$ \\
\hline GQ536 & $N 2 ; g z 158[p r a b-11.1: \because g f p]$ \\
\hline GQ537 & $N 2 ; g z 159[p r a b-5:: g f p]$ \\
\hline GQ538 & $N 2 ; g z 160[p r a b-6.1: \because g f p]$ \\
\hline GQ539 & $N 2 ; g z 161[p r a b-6.2: \because g f p]$ \\
\hline GQ540 & $N 2 ; g z 162[p r a b-7: \because g f p]$ \\
\hline GQ541 & N2;gz163[prab-10::gfp] \\
\hline GQ542 & $N 2 ; g z 164[p r a b-11.2: \because g f p]$ \\
\hline GQ543 & $N 2 ; g z 165[p r a b-27: \because g f p]$ \\
\hline GQ544 & $N 2 ; g z 166[p r a b-35: \because g f p]$ \\
\hline GQ545 & N2;gz167[prab-37.1::gfp] \\
\hline GQ546 & N2;gz168[pglo-1::gfp] \\
\hline GQ547 & $N 2 ; g z 169[p r a b-37.2: \because g f p]$ \\
\hline GQ548 & $N 2 ; g z 170[p r a b-3:: g f p]$ \\
\hline GQ549 & N2;gz171[prab-3::mcherry-rab-1; prab-3::mannsII-yfp] \\
\hline GQ550 & N2;gz172[prab-3::tagRFP-rab-8; prab-3::mannsII-yfp] \\
\hline GQ551 & N2;gz173[prab-3::tagRFP-rab-11.1; prab-3::mannsII-yfp] \\
\hline GQ552 & N2;gz174[prab-3::tagRFP-rab-33 prab-3::mannsII-yfp] \\
\hline GQ553 & N2;gz175[prab-3::tagRFP-rab-35; prab-3::mannsII-yfp] \\
\hline GQ554 & N2;gz176[prab-3::tagRFP-glo-1; prab-3::mannsII-yfp] \\
\hline GQ555 & N2;gz177[prab-3::tagRFP-rab-y3; prab-3::mannsII-yfp] \\
\hline GQ556 & N2;gz178[prab-3::tagRFP-rab-37.1; prab-3::mannsII-yfp] \\
\hline GQ557 & N2;gz179[prab-3::tagRFP-rab-37.2; prab-3::mannsII-yfp] \\
\hline GQ558 & N2;gz180[prab-3::mcherry-rab-3; prab-3::mannsII-yfp] \\
\hline GQ559 & N2;gz181[prab-3::mcherry-rab-5; prab-3::mannsII-yfp] \\
\hline GQ560 & N2;gz182[prab-3::mcherry-rab-7; prab-3::mannsII-yfp] \\
\hline GQ561 & N2;gz183[prab-3::tagRFP-rab-27; prab-3::mannsII-yfp] \\
\hline GQ562 & N2;gz184[prab-3::tagRFP-rab-39; prab-3::mannsII-yfp] \\
\hline GQ563 & N2;gz185[prab-3::tagRFP-rab-y1; prab-3::mannsII-yfp] \\
\hline GQ564 & N2;gz186[prab-3::tagRFP-rab-y2; prab-3::mannsII-yfp] \\
\hline GQ565 & N2;gz187[prab-3::mcherry-rab-6.1; prab-3::mannsII-yfp] \\
\hline GQ566 & N2;gz188[prab-3::mcherry-rab-6.2; prab-3::mannsII-yfp] \\
\hline
\end{tabular}




\begin{tabular}{|c|c|}
\hline GQ567 & N2;gz189[prab-3::tagRFP-rab-18; prab-3::mannsII-yfp] \\
\hline GQ568 & N2;gz190[prab-3::tagRFP-rab-19; prab-3::mannsII-yfp] \\
\hline GQ569 & N2;gz191[prab-28::mcherry-rab-28; prab-28::gfp-rab-6.1] \\
\hline GQ570 & N2;gz192[prab-30::mcherry-rab-30; prab-30::gfp-rab-6.1] \\
\hline GQ571 & N2;gz193[prab-28::mcherry-rab-28; prab-3::ctns-1-yfp] \\
\hline GQ572 & N2;gz194[prab-14::mcherry-rab-14; prab-3::gfp-rab-6.1] \\
\hline GQ589 & N2;gz144[prab-8::gfp] II \\
\hline GQ590 & $\mathrm{N} 2 ; \mathrm{gz} 145[\mathrm{prab}-28:: \mathrm{gfp}] \mathrm{II}$ \\
\hline GQ591 & N2;gz146[prab-30::gfp] II \\
\hline GQ592 & N2;gz147[prab-19::gfp] II \\
\hline GQ593 & N2;gz148[prab-18.1::gfp] II \\
\hline GQ594 & $\mathrm{N} 2 ; \mathrm{gz} 149[\mathrm{prab}-18.2:: \mathrm{gfp}] \mathrm{II}$ \\
\hline GQ595 & $\mathrm{N} 2 ; \mathrm{gz} 150[\mathrm{prab}-21:: \mathrm{gfp}] \mathrm{II}$ \\
\hline GQ596 & $\mathrm{N} 2 ; \mathrm{gz} 151[\mathrm{prab}-33:: \mathrm{gfp}] \mathrm{II}$ \\
\hline GQ597 & N2;gz152[prab-y2::gfp] II \\
\hline GQ598 & $\mathrm{N} 2 ; \mathrm{gz} 153[$ prab-y3::gfp] II \\
\hline GQ599 & N2;gz154[prab-y5::gfp] II \\
\hline GQ600 & N2;gz155[prab-39::gfp] II \\
\hline GQ601 & N2;gz156[prab-y6::gfp] II \\
\hline GQ602 & N2;gz157[prab-y4::gfp] II \\
\hline GQ603 & N2;gz158[prab-11.1::gfp] II \\
\hline GQ604 & N2;gz159[prab-5::gfp] II \\
\hline GQ605 & $\mathrm{N} 2 ; \mathrm{gz} 160[$ prab-6.1::gfp] II \\
\hline GQ606 & $\mathrm{N} 2 ; \mathrm{gz} 161[$ prab-6.2::gfp] II \\
\hline GQ607 & N2;gz162[prab-7::gfp] II \\
\hline GQ608 & N2;gz163[prab-10::gfp] II \\
\hline GQ609 & $\mathrm{N} 2 ; \mathrm{gz} 164[$ prab-11.2::gfp] II \\
\hline GQ610 & N2;gz165[prab-27::gfp] II \\
\hline GQ611 & N2;gz166[prab-35::gfp] II \\
\hline GQ612 & N2;gz167[prab-37.1::gfp] II \\
\hline GQ613 & N2;gz168[pglo-1::gfp] II \\
\hline GQ614 & N2;gz169[prab-37.2::gfp] II \\
\hline GQ615 & N2;gz170[prab-3::gfp] II \\
\hline GQ616 & N2;gz171[prab-3::mcherry-rab-1; prab-3::mannsII-yfp] II \\
\hline GQ617 & N2;gz172[prab-3::tagRFP-rab-8; prab-3::mannsII-yfp] II \\
\hline GQ618 & N2;gz173[prab-3::tagRFP-rab-11.1; prab-3::mannsII-yfp] II \\
\hline GQ619 & N2;gz174[prab-3::tagRFP-rab-33 prab-3::mannsII-yfp] II \\
\hline GQ620 & N2;gz175[prab-3::tagRFP-rab-35; prab-3::mannsII-yfp] II \\
\hline GQ621 & N2;gz176[prab-3::tagRFP-glo-1; prab-3::mannsII-yfp] II \\
\hline GQ622 & N2;gz177[prab-3::tagRFP-rab-y3; prab-3::mannsII-yfp] II \\
\hline GQ623 & N2;gz178[prab-3::tagRFP-rab-37.1; prab-3::mannsII-yfp] II \\
\hline GQ624 & N2;gz179[prab-3::tagRFP-rab-37.2; prab-3::mannsII-yfp] II \\
\hline GQ625 & N2;gz180[prab-3::mcherry-rab-3; prab-3::mannsII-yfp] II \\
\hline GQ626 & N2;gz181[prab-3::mcherry-rab-5; prab-3::mannsII-yfp] II \\
\hline GQ627 & N2;gz182[prab-3::mcherry-rab-7; prab-3::mannsII-yfp] II \\
\hline GQ628 & N2;gz183[prab-3::tagRFP-rab-27; prab-3::mannsII-yfp] II \\
\hline GQ629 & N2;gz184[prab-3::tagRFP-rab-39; prab-3::mannsII-yfp] II \\
\hline GQ630 & N2;gz185[prab-3::tagRFP-rab-y1; prab-3::mannsII-yfp] II \\
\hline GQ631 & N2;gz186[prab-3::tagRFP-rab-y2; prab-3::mannsII-yfp] II \\
\hline GQ632 & N2;gz187[prab-3::mcherry-rab-6.1; prab-3::mannsII-yfp] II \\
\hline GQ633 & N2;gz188[prab-3::mcherry-rab-6.2; prab-3::mannsII-yfp] II \\
\hline GQ634 & N2;gz189[prab-3::tagRFP-rab-18; prab-3::mannsII-yfp] II \\
\hline GQ635 & N2;gz190[prab-3::tagRFP-rab-19; prab-3::mannsII-yfp] II \\
\hline GQ636 & N2;gz191[prab-28::mcherry-rab-28; prab-28::gfp-rab-6.1] II \\
\hline GQ637 & N2;gz192[prab-30::mcherry-rab-30; prab-30::gfp-rab-6.1] II \\
\hline GQ638 & N2;gz193[prab-28::mcherry-rab-28; prab-3::ctns-1-yfp] II \\
\hline GQ639 & N2;gz194[prab-14::mcherry-rab-14; prab-3::gfp-rab-6.1] II \\
\hline
\end{tabular}




\subsection{Injection mixes for extrachromosomal arrays}

All injection mixes used to generate transgenic lines bearing extrachromosomal arrays are listed in table XII.3

Table XII.3. List of all injection mixes used to generate transgenic lines

\begin{tabular}{|c|c|c|}
\hline $\begin{array}{l}\text { Array } \\
\text { Name }\end{array}$ & Plasmids of interest & Markers \\
\hline gz130 & $5 \mathrm{ng} / \mu \mathrm{l}$ punc-129::mcherry-rab-10 & 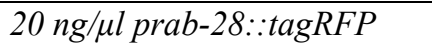 \\
\hline gz131 & $20 \mathrm{ng} / \mu \mathrm{l}$ prab-3::tagRFP-tbc-4 & $20 n g / \mu l p t t x-3: \because g f p$ \\
\hline gz132 & $20 \mathrm{ng} / \mu \mathrm{l}$ prab-3::tagRFP-tbc-4 R155A & $20 \mathrm{ng} / \mu \mathrm{l}$ prab-28::tagRFP \\
\hline gz133 & $20 \mathrm{ng} / \mathrm{\mu l}$ punc-129::rde-4 & 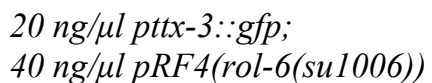 \\
\hline gz134 & $\begin{array}{l}10 \mathrm{ng} / \mu \mathrm{l} \text { prab-3::cb-5-gfp; } \\
3 \mathrm{ng} / \mu \mathrm{l} \text { prab-3::mcherry-rab-10}\end{array}$ & $40 \mathrm{ng} / \mu \mathrm{l}$ pRF4(rol-6(su1006)) \\
\hline gz135 & 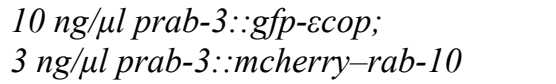 & $40 \mathrm{ng} / \mu \mathrm{l} \mathrm{pRF4(rol-6(su1006))}$ \\
\hline gz136 & $\begin{array}{l}10 \mathrm{ng} / \mu \mathrm{l} \text { prab-3::mannsII-yfp; } \\
3 \mathrm{ng} / \mu \mathrm{l} \text { prab-3::mcherry-rab-10}\end{array}$ & $40 \mathrm{ng} / \mu \mathrm{l} \mathrm{pRF4(rol-6(su1006))}$ \\
\hline gz137 & $\begin{array}{l}10 \mathrm{ng} / \mu \mathrm{l} \text { prab-3::gfp-2xfyve domain; } \\
3 \mathrm{ng} / \mu \mathrm{l} \text { prab-3::mcherry-rab-10}\end{array}$ & $40 \mathrm{ng} / \mu \mathrm{l} \mathrm{pRF4(rol-6(su1006))}$ \\
\hline gz138 & $\begin{array}{l}3 \mathrm{ng} / \mu \mathrm{l} \text { prab-3::tagRFP-syx-6; } \\
3 \mathrm{ng} / \mu \mathrm{l} \text { prab-3::gfp-rab-10}\end{array}$ & $30 \mathrm{ng} / \mu \mathrm{l}$ pRF4(rol-6(su1006)) \\
\hline gz139 & $\begin{array}{l}20 \mathrm{ng} / \mu \mathrm{l} \text { prab-3::tagRFP-tbc-4; } \\
3 \mathrm{ng} / \mu \mathrm{l} \text { prab-3::mcherry-rab-10}\end{array}$ & $30 \mathrm{ng} / \mu \mathrm{l} \mathrm{pRF4(rol-6(su1006))}$ \\
\hline gz140 & 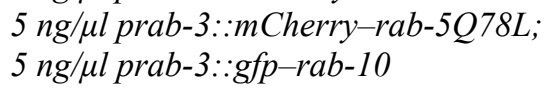 & $30 \mathrm{ng} / \mu \mathrm{l}$ pRF4(rol-6(su1006)) \\
\hline gz141 & 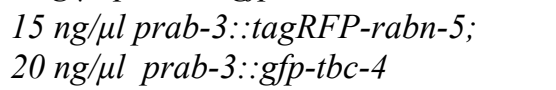 & $30 \mathrm{ng} / \mu \mathrm{l}$ pRF4(rol-6(su1006)) \\
\hline gz142 & 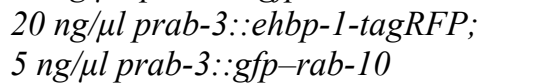 & $20 n g / \mu l p t t x-3: \because g f p$ \\
\hline gz143 & $\begin{array}{l}20 \mathrm{ng} / \mu \mathrm{l} \text { prab-3::yfp-apt-9; } \\
5 \mathrm{ng} / \mu \mathrm{l} \text { prab-3::mcherry-rab-10}\end{array}$ & 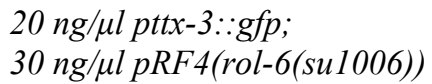 \\
\hline gz144 & $30 \mathrm{ng} / \mu \mathrm{l}$ pPD115.62 prab-8::gfp & $40 \mathrm{ng} / \mu \mathrm{l} \mathrm{pRF4(rol-6(su1006))}$ \\
\hline gz145 & $30 \mathrm{ng} / \mu \mathrm{l}$ pPD115.62 prab-28::gfp & $40 \mathrm{ng} / \mu \mathrm{l} \mathrm{pRF4(rol-6(su1006))}$ \\
\hline gz146 & $30 \mathrm{ng} / \mu \mathrm{l}$ pPD115.62 prab-30.:gfp & $40 \mathrm{ng} / \mu \mathrm{l}$ pRF4(rol-6(su1006)) \\
\hline gz147 & 30 ng/ul pPD115.62 prab-19::gfp & $40 \mathrm{ng} / \mu \mathrm{l}$ pRF4(rol-6(su1006)) \\
\hline gz148 & $30 \mathrm{ng} / \mu \mathrm{l}$ pPD115.62 prab-18.1::gfp & $40 \mathrm{ng} / \mu \mathrm{l} \mathrm{pRF4(rol-6(su1006))}$ \\
\hline gz149 & $30 \mathrm{ng} / \mu \mathrm{l}$ pPD115.62 prab-18.2::gfp & $40 \mathrm{ng} / \mu \mathrm{l}$ pRF4(rol-6(su1006)) \\
\hline gz150 & $30 \mathrm{ng} / \mu \mathrm{l}$ pPD115.62 prab-21::gfp & $40 \mathrm{ng} / \mu \mathrm{l}$ pRF4(rol-6(su1006)) \\
\hline gz151 & $30 \mathrm{ng} / \mu \mathrm{l}$ pPD115.62 prab-33::gfp & $40 \mathrm{ng} / \mu \mathrm{l} \mathrm{pRF4(rol-6(su1006))}$ \\
\hline gz152 & $30 \mathrm{ng} / \mu \mathrm{l}$ pPD115.62 prab-y2::gfp & $45 \mathrm{ng} / \mu \mathrm{l}$ pRF4(rol-6(su1006)) \\
\hline gz153 & $30 \mathrm{ng} / \mu \mathrm{l}$ pPD115.62 prab-y3::gfp & $35 \mathrm{ng} / \mu \mathrm{l}$ pRF4(rol-6(su1006)) \\
\hline gz154 & $30 \mathrm{ng} / \mu \mathrm{l}$ pPD115.62 prab-y5::gfp & $45 \mathrm{ng} / \mu \mathrm{l} \mathrm{pRF4(rol-6(su1006))}$ \\
\hline gz155 & $30 \mathrm{ng} / \mu \mathrm{l}$ pPD115.62 prab-39::gfp & $35 \mathrm{ng} / \mu \mathrm{l}$ pRF4(rol-6(su1006)) \\
\hline gz156 & $30 \mathrm{ng} / \mu \mathrm{l}$ pPD115.62 prab-y6::gfp & $35 \mathrm{ng} / \mu \mathrm{l}$ pRF4(rol-6(su1006)) \\
\hline gz157 & $30 \mathrm{ng} / \mu \mathrm{l}$ pPD115.62 prab-y4:.gfp & $35 \mathrm{ng} / \mu \mathrm{l} \mathrm{pRF4(rol-6(su1006))}$ \\
\hline gz158 & $30 \mathrm{ng} / \mu \mathrm{l}$ pPD115.62 prab-11.1::gfp & $30 \mathrm{ng} / \mu \mathrm{l} \mathrm{pRF4(rol-6(su1006))}$ \\
\hline gz159 & $30 \mathrm{ng} / \mu \mathrm{l}$ pPD115.62 prab-5::gfp & $40 \mathrm{ng} / \mu \mathrm{l} \mathrm{pRF4(rol-6(su1006))}$ \\
\hline gz160 & $30 \mathrm{ng} / \mu \mathrm{l}$ pPD115.62 prab-6.1::gfp & $40 \mathrm{ng} / \mu \mathrm{l} \mathrm{pRF4(rol-6(su1006))}$ \\
\hline gz161 & $30 \mathrm{ng} / \mu \mathrm{l}$ pPD115.62 prab-6.2::gfp & $40 \mathrm{ng} / \mu \mathrm{l}$ pRF4(rol-6(su1006)) \\
\hline gz162 & $30 \mathrm{ng} / \mu \mathrm{l}$ pPD115.62 prab-7::gfp & $40 \mathrm{ng} / \mu \mathrm{l} \mathrm{pRF4(rol-6(su1006))}$ \\
\hline gz163 & $30 \mathrm{ng} / \mu \mathrm{l}$ pPD115.62 prab-10::gfp & $40 \mathrm{ng} / \mu \mathrm{l}$ pRF4(rol-6(su1006)) \\
\hline gz164 & $30 \mathrm{ng} / \mu \mathrm{l}$ pPD115.62 prab-11.2::gfp & $40 \mathrm{ng} / \mu \mathrm{l}$ pRF4(rol-6(su1006)) \\
\hline gz165 & 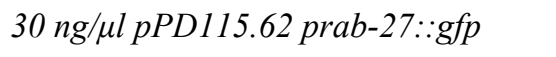 & $40 \mathrm{ng} / \mu \mathrm{l}$ pRF4(rol-6(su1006)) \\
\hline
\end{tabular}




\begin{tabular}{|c|c|}
\hline gz166 & $30 \mathrm{ng} / \mu \mathrm{lpPD1} 15.62 \mathrm{prab}-35: \because g f p$ \\
\hline gz167 & $30 \mathrm{ng} / \mu \mathrm{l}$ pPD115.62 prab-37.1::gfp \\
\hline gz168 & $30 \mathrm{ng} / \mu \mathrm{l}$ pPD115.62 pglo-1::gfp \\
\hline gz169 & $30 \mathrm{ng} / \mu \mathrm{l}$ pPD115.62 prab-37.2::gfp \\
\hline gz170 & $30 \mathrm{ng} / \mu \mathrm{l}$ prab-3::gfp \\
\hline gz171 & $\begin{array}{l}5 \mathrm{ng} / \mu \mathrm{l} \text { prab-3::mcherry-rab-1 } \\
10 \mathrm{ng} / \mu \mathrm{l} \text { prab-3::mannsII-yfp }\end{array}$ \\
\hline gz172 & $\begin{array}{l}5 \mathrm{ng} / \mu \mathrm{l} \text { prab-3::tagRFP-rab-8 } \\
10 \mathrm{ng} / \mu \mathrm{l} \text { prab-3::mannsII-yfp }\end{array}$ \\
\hline gz173 & $\begin{array}{l}5 \mathrm{ng} / \mu \mathrm{l} \text { prab-3::tagRFP-rab-11.1 } \\
10 \mathrm{ng} / \mu \mathrm{l} \text { prab-3::mannsII-yfp }\end{array}$ \\
\hline gz174 & $\begin{array}{l}5 \mathrm{ng} / \mu \mathrm{l} \text { prab-3::tagRFP-rab-33 } \\
10 \mathrm{ng} / \mathrm{\mu l} \text { prab-3::mannsII-yfp }\end{array}$ \\
\hline gz175 & $\begin{array}{l}5 \mathrm{ng} / \mu \mathrm{l} \text { prab-3::tagRFP-rab-35 } \\
10 \mathrm{ng} / \mu \mathrm{l} \text { prab-3::mannsII-yfp }\end{array}$ \\
\hline gz176 & $\begin{array}{l}5 \mathrm{ng} / \mu \mathrm{l} \text { prab-3::tagRFP-glo-1 } \\
10 \mathrm{ng} / \text { ul prab-3::mannsII-yfp }\end{array}$ \\
\hline gz177 & $\begin{array}{l}5 \mathrm{ng} / \mu \mathrm{l} \text { prab-3::tagRFP-rab-y3 } \\
10 \mathrm{ng} / \mu \mathrm{l} \text { prab-3::mannsII-yfp }\end{array}$ \\
\hline gz178 & $\begin{array}{l}5 \mathrm{ng} / \mu \mathrm{l} \text { prab-3::tagRFP-rab-37.1 } \\
10 \mathrm{ng} / \mu \mathrm{l} \text { prab-3::mannsII-yfp }\end{array}$ \\
\hline gz179 & $\begin{array}{l}5 \mathrm{ng} / \mu \mathrm{l} \text { prab-3::tagRFP-rab-37.2 } \\
10 \mathrm{ng} / \mu \mathrm{l} \text { prab-3::mannsII-yfp }\end{array}$ \\
\hline gz180 & $\begin{array}{l}5 \mathrm{ng} / \mu \mathrm{l} \text { prab-3::mcherry-rab-3 } \\
10 \mathrm{ng} / \mu \mathrm{l} \text { prab-3::mannsII-yfp }\end{array}$ \\
\hline gz181 & $\begin{array}{l}5 \mathrm{ng} / \mu \mathrm{l} \text { prab-3::mcherry-rab-5 } \\
10 \mathrm{ng} / \mu \mathrm{l} \text { prab-3::mannsII-yfp }\end{array}$ \\
\hline gz182 & $\begin{array}{l}5 \mathrm{ng} / \mu \mathrm{l} \text { prab-3::mcherry-rab-7 } \\
10 \mathrm{ng} / \mu \mathrm{l} \text { prab-3::mannsII-yfp }\end{array}$ \\
\hline gz183 & $\begin{array}{l}5 \mathrm{ng} / \mu \mathrm{l} \text { prab-3::tagRFP-rab-27 } \\
10 \mathrm{ng} / \mu \mathrm{l} \text { prab-3::mannsII-yfp }\end{array}$ \\
\hline gz184 & $\begin{array}{l}5 \mathrm{ng} / \mu \mathrm{l} \text { prab-3::tagRFP-rab-39 } \\
10 \mathrm{ng} / \mu \mathrm{l} \text { prab-3::mannsII-yfp }\end{array}$ \\
\hline gz185 & $\begin{array}{l}5 \mathrm{ng} / \mu \mathrm{l} \text { prab-3::tagRFP-rab-yl } \\
10 \mathrm{ng} / \mu \mathrm{l} \text { prab-3::mannsII-yfp }\end{array}$ \\
\hline gz186 & $\begin{array}{l}5 \mathrm{ng} / \mu \mathrm{l} \text { prab-3::tagRFP-rab-y2 } \\
10 \mathrm{ng} / \mu \mathrm{l} \text { prab-3::mannsII-yfp }\end{array}$ \\
\hline gz187 & $\begin{array}{l}5 \mathrm{ng} / \mu \mathrm{l} \text { prab-3::mcherry-rab-6.1 } \\
10 \mathrm{ng} / \mu \mathrm{l} \text { prab-3::mannsII-yfp }\end{array}$ \\
\hline gz188 & $\begin{array}{l}5 \mathrm{ng} / \mu \mathrm{l} \text { prab-3::mcherry-rab-6.2 } \\
10 \mathrm{ng} / \mu \mathrm{l} \text { prab-3::mannsII-yfp }\end{array}$ \\
\hline gz189 & $\begin{array}{l}5 \mathrm{ng} / \mu \mathrm{l} \text { prab-3::tagRFP-rab-18 } \\
10 \mathrm{ng} / \mu \mathrm{l} \text { prab-3::mannsII-yfp }\end{array}$ \\
\hline gz190 & $\begin{array}{l}5 \mathrm{ng} / \mu \mathrm{l} \text { prab-3::tagRFP-rab-19 } \\
10 \mathrm{ng} / \mu \mathrm{l} \text { prab-3::mannsII-yfp }\end{array}$ \\
\hline gz191 & $\begin{array}{l}5 \mathrm{ng} / \mu \mathrm{l} \text { prab-28::mcherry-rab-28 } \\
5 \mathrm{ng} / \mu \mathrm{l} \text { prab-28::gfp-rab-6.1 }\end{array}$ \\
\hline gz192 & $\begin{array}{l}5 \mathrm{ng} / \mu \mathrm{l} \text { prab-30::mcherry-rab-30 } \\
5 \mathrm{ng} / \mu \mathrm{l} \text { prab-30::gfp-rab-6.1 }\end{array}$ \\
\hline gz193 & $\begin{array}{l}5 \mathrm{ng} / \mu \mathrm{l} \text { prab-28::mcherry-rab-28 } \\
5 \mathrm{ng} / \mu \mathrm{l} \text { prab-3::ctns-1-yfp }\end{array}$ \\
\hline gz194 & $\begin{array}{l}5 \mathrm{ng} / \mu \mathrm{l} \text { prab-14::mcherry-rab-14 } \\
5 \mathrm{ng} / \mu \mathrm{l} \text { prab-3::gfp-rab-6.1 }\end{array}$ \\
\hline
\end{tabular}

40 ng/ $\mu$ l pRF4(rol-6(su1006))

$40 \mathrm{ng} / \mu \mathrm{l} \mathrm{pRF4(rol-6(su1006))}$

$40 \mathrm{ng} / \mu \mathrm{l} \mathrm{pRF4(rol-6(su1006))}$

$35 \mathrm{ng} / \mu \mathrm{l}$ pRF4(rol-6(su1006))

$35 \mathrm{ng} / \mu \mathrm{l} \mathrm{pRF4(rol-6(su1006))}$

20 ng/ul pttx-3::gfp;

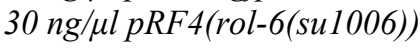

$20 \mathrm{ng} / \mu \mathrm{l}$ pttx-3::gfp;

$30 \mathrm{ng} / \mu \mathrm{l} \mathrm{pRF4(rol-6(su1006))}$

$20 \mathrm{ng} / \mu \mathrm{l}$ pttx-3::gfp;

$30 \mathrm{ng} / \mu \mathrm{l} \mathrm{pRF4(rol-6(su1006))}$

$20 \mathrm{ng} / \mu \mathrm{l}$ pttx-3::gfp;

$30 \mathrm{ng} / \mu \mathrm{l} \mathrm{pRF4(rol-6(su1006))}$

$20 \mathrm{ng} / \mu \mathrm{l}$ pttx-3::gfp;

$30 \mathrm{ng} / \mu \mathrm{l} \mathrm{pRF4(rol-6(su1006))}$

$20 \mathrm{ng} / \mu \mathrm{l}$ pttx-3::gfp;

$30 \mathrm{ng} / \mu \mathrm{l} \mathrm{pRF4(rol-6(su1006))}$

$20 \mathrm{ng} / \mu \mathrm{l}$ pttx-3::gfp;

$30 \mathrm{ng} / \mu \mathrm{l} \mathrm{pRF4(rol-6(su1006))}$

$20 \mathrm{ng} / \mu \mathrm{l}$ pttx-3::gfp;

$30 \mathrm{ng} / \mu \mathrm{l} \mathrm{pRF4(rol-6(su1006))}$

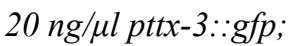

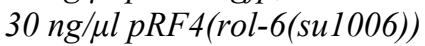

$20 \mathrm{ng} / \mu \mathrm{l}$ pttx-3::gfp;

$30 \mathrm{ng} / \mu \mathrm{l} \mathrm{pRF4(rol-6(su1006))}$

20 ng/ul pttx-3::gfp;

$30 \mathrm{ng} / \mu \mathrm{l} \mathrm{pRF4(rol-6(su1006))}$

$20 \mathrm{ng} / \mu \mathrm{l}$ pttx-3::gfp;

$30 \mathrm{ng} / \mu \mathrm{l} \mathrm{pRF4(rol-6(su1006))}$

$20 \mathrm{ng} / \mu \mathrm{l}$ pttx-3::gfp;

$30 \mathrm{ng} / \mu \mathrm{l} \mathrm{pRF4(rol-6(su1006))}$

$20 \mathrm{ng} / \mu \mathrm{l}$ pttx-3::gfp;

$30 \mathrm{ng} / \mu \mathrm{l} \mathrm{pRF4(rol-6(su1006))}$

$20 \mathrm{ng} / \mu \mathrm{l}$ pttx-3::gfp;

$30 \mathrm{ng} / \mu \mathrm{l} \mathrm{pRF4(rol-6(su1006))}$

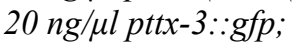

$30 \mathrm{ng} / \mu \mathrm{l} \mathrm{pRF4(rol-6(su1006))}$

$20 \mathrm{ng} / \mu \mathrm{l}$ pttx-3::gfp;

$30 \mathrm{ng} / \mu \mathrm{l} \mathrm{pRF4(rol-6(su1006))}$

$20 \mathrm{ng} / \mu \mathrm{l}$ pttx-3::gfp;

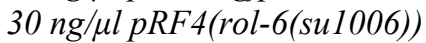

20 ng/ul pttx-3::gfp;

$30 \mathrm{ng} / \mu \mathrm{l} \mathrm{pRF4(rol-6(su1006))}$

20 ng/ul pttx-3::gfp;

$30 \mathrm{ng} / \mu \mathrm{l} \mathrm{pRF4(rol-6(su1006))}$

$30 \mathrm{ng} / \mu \mathrm{l} \mathrm{pRF4(rol-6(su1006))}$

$30 \mathrm{ng} / \mu \mathrm{l}$ pRF4(rol-6(su1006))

$45 \mathrm{ng} / \mu \mathrm{l}$ pRF4(rol-6(su1006))

40 ng/ $\mu l$ pRF4(rol-6(su1006)) 


\subsection{Deletion alleles}

All deletion alleles were genotyped using worm PCR. Table XII.4 lists all the deletion alleles used in this study along with the respective primers used for genotyping. Expected band sizes for wild type (WT) are shown.

Table XII.4. List of all deletion alleles with respective worm PCR primers used in this study

\begin{tabular}{|c|c|c|c|c|c|}
\hline $\begin{array}{l}\text { Deletion strains } \\
\text { used in study }\end{array}$ & $\begin{array}{l}\text { Size of } \\
\text { deletion } \\
\text { (bp) }\end{array}$ & $\begin{array}{l}\text { Outside deletion } \\
\text { primers }\end{array}$ & $\begin{array}{l}\text { PCR } \\
\text { band } \\
\text { size } \\
\text { in WT } \\
\text { (bp) }\end{array}$ & $\begin{array}{l}\text { Inside deletion } \\
\text { primers }\end{array}$ & $\begin{array}{l}\text { PCR } \\
\text { band } \\
\text { size } \\
\text { in WT } \\
\text { (bp) }\end{array}$ \\
\hline$r a b-2$ (nu415) & 201 & oGQ1278/oGQ1280 & 1755 & oGQ1278/oGQ1283 & 1179 \\
\hline$r a b-6.2(o k 2254)$ & 847 & oGQ977/oGQ978 & 1736 & oGQ979/oGQ980 & 673 \\
\hline$r a b-8(\operatorname{tm} 2991)$ & 314 & oGQ1288/oGQ1290 & 2198 & oGQ1288/oGQ1293 & 1638 \\
\hline$r a b-10(o k 1494)$ & 663 & oGQ1185/oGQ1188 & 1527 & oGQ1186/oGQ1188 & 911 \\
\hline $\begin{array}{l}r a b- \\
11.2(\operatorname{tm} 2081)\end{array}$ & 647 & oGQ1300/oGQ1302 & 1790 & oGQ1300/oGQ1305 & 1140 \\
\hline$r a b-14(\operatorname{tm} 2095)$ & 702 & oGQ362/oGQ363 & 1345 & oGQ374/oGQ363 & 589 \\
\hline$r a b-18(o k 2020)$ & 1316 & oGQ1194/oGQ1195 & 2874 & oGQ1193/oGQ1198 & 1329 \\
\hline$r a b-19(o k 1845)$ & 1257 & oGQ1295/oGQ591 & 1931 & oGQ1298/oGQ1297 & 1286 \\
\hline$r a b-21(o k 1879)$ & 1096 & oGQ1307/oGQ264 & 1686 & oGQ1310/oGQ264 & 1125 \\
\hline$r a b-27(t m 2306)$ & 252 & oGQ265/oGQ1314 & 1946 & oGQ265/oGQ1317 & 1292 \\
\hline$r a b-28(o k 3424)$ & 393 & oGQ815/oGQ819 & 2739 & oGQ815/oGQ1078 & 1935 \\
\hline$r a b-33(o k 1561)$ & 742 & oGQ1319/oGQ1321 & 2246 & oGQ1319/oGQ1399 & 1937 \\
\hline$r a b-35(\operatorname{tm} 2058)$ & 480 & oGQ1322/oGQ1324 & 2103 & oGQ1322/oGQ1327 & 1262 \\
\hline$r a b-37(t m 2089)$ & 1063 & oGQ1329/oGQ1331 & 2458 & oGQ1328/oGQ1333 & 1244 \\
\hline$r a b-39(t m 2466)$ & 189 & oGQ1334/oGQ1336 & 1343 & oGQ1334/oGQ1339 & 608 \\
\hline rab-yl(ok1356) & 1349 & oGQ1352/oGQ1354 & 2095 & oGQ1353/oGQ1357 & 1045 \\
\hline$r a b-y 3(o k 1871)$ & 1668 & oGQ1267/oGQ1268 & 2639 & oGQ1267/oGQ1271 & 1467 \\
\hline eri-1 (mg366) & $\begin{array}{c}23 \\
\text { insertion }\end{array}$ & oGQ1358/oGQ1359 & 155 & & \\
\hline$t b c-2(\operatorname{tm} 2241)$ & 230 & oGQ1556/oGQ1689 & 677 & oGQ1688/oGQ1689 & 498 \\
\hline$t b c-4(o k 2928)$ & 821 & oGQ1492/oGQ1493 & 1621 & oGQ1492/oGQ1494 & 787 \\
\hline$t b c-4(\operatorname{tm} 3255)$ & 249 & oGQ1492/oGQ1493 & 1621 & oGQ1492/oGQ1815 & 1033 \\
\hline rabn-5(ok1555) & 841 & oGQ2020/oGQ2022 & 2255 & oGQ1475/oGQ1476 & 1914 \\
\hline
\end{tabular}

\subsection{DNA sequences of primers:}

Table XII.5 lists the DNA sequences of all primers that were used in this study. All primers are numbered with an oGQ number, which is an internal reference number specific to the Eimer lab.

Table XII.5 List of DNA sequences of all primers used

\begin{tabular}{ll}
\hline Primer number & Primer sequence \\
\hline oGQ230 & GGGCATATGGCCATGGCGGCTGGCGGACAACCT \\
oGQ231 & GGGGGATCCCTCGAGTTAGCAATTGCATTGCTGTTG \\
oGQ244 & GTCGGAAAAAACTGTCTTCTTCATCAGTTCACA \\
oGQ245 & AAGAAGACAGTTTTTTCCGACACCCATATCACC \\
oGQ246 & GTCAGCGGACTGAAGATCAAGCTTCAAATCTGG \\
oGQ247 & CTTGATCTTCAGTCCGCTGACCTCAATAATCCT \\
oGQ259 & GGATCCCATATGGCAAAAACTTACGACTAC \\
oGQ260 & GGGGAATTCTTAAAGCAAATTGCAGCTCCA \\
oGQ261 & GGATCCCATATGGCTCGCCGACCGTATGAC
\end{tabular}




\begin{tabular}{|c|c|}
\hline oGQ262 & CCCGAATTCCTAGCAGCATCCTCCACTGCT \\
\hline oGQ263 & GGATCCCATATGCTCGAAACCAACGTGGAT \\
\hline oGQ264 & CCCGAATTCTCAGCGACAGCACTTTTTACT \\
\hline oGQ264 & CCCGAATTCTCAGCGACAGCACTTTTTACT \\
\hline oGQ265 & GGATCCGCCATGGGTGACTACGACTATCTC \\
\hline oGQ266 & GGGCTCGAGTCAGCAATTTGCACATATGGA \\
\hline oGQ268 & GGATCCCATATGTCCGACGACAGTTCAAGC \\
\hline oGQ269 & CCCGAATTCCTAGCATCCACACATTCCGCC \\
\hline oGQ270 & GGATCCGCCATGGAAACAAACTTCATTGGTG \\
\hline oGQ271 & CCCCTCGAGTCAACATCCGCAAGCTCCTGC \\
\hline oGQ272 & GGATCCCATATGGCGGGAACTCGGGATTAC \\
\hline oGQ273 & GGGGAATTCTTATCCACATTTGCACTTCTT \\
\hline oGQ274 & GGATCCCATATGCTACTTGGTGACAGCTGC \\
\hline oGQ275 & GGGGAATTCTCAATTAAACGTGCAACATCT \\
\hline oGQ276 & GGATCCCATATGCCATATCAAACAAACGAA \\
\hline oGQ277 & GGATCCGCCATGGCAACAATGGGAGAAGACGAG \\
\hline oGQ278 & GGGGAATTCTTAAGTAATGGAGCATACAGA \\
\hline oGQ279 & AGATCTCATATGTCGGAGCATCATGTGAAC \\
\hline oGQ280 & CCCGAATTCTCAGCAGCAGAATCCCTCTTC \\
\hline oGQ281 & GGATCCCATATGGAGGATTACAAGTATCTA \\
\hline oGQ282 & GGGCTCGAGCTAAGATTGTCGAGTACAACA \\
\hline oGQ283 & GGATCCCATATGTCGGGAACCAGAAAGAAG \\
\hline oGQ284 & CCCCTCGAGTTAACAATTGCATCCCGAATT \\
\hline oGQ285 & GGATCCCATATGGCCGCCCGAAACGCAGGA \\
\hline oGQ286 & CCCGAATTCTTATTTACAGCATGAACCCTTTTG \\
\hline oGQ362 & GGGTAGATGGTCGAATAGTGA \\
\hline oGQ363 & TGTAGGTAGAACGGCGAGTGA \\
\hline oGQ374 & CATTTGTCTCTCTCTCCCCGT \\
\hline oGQ478 & CCATGGTACCGGTACATATGACAGAACCGTCGGCTAGC \\
\hline oGQ479 & GGGAATTCGTTACTTATCCTGCGTTGCAAAG \\
\hline oGQ484 & CCCCAAGCTTCGGCTAGTTTATCCACTGAGA \\
\hline oGQ485 & CCCTACCGGTACCGTTTTTGCCATTTTATTGGAGTGAGT \\
\hline oGQ486 & GCGGAAGCTTGGGTTCATTCACTTACCATCC \\
\hline oGQ487 & CCCTACCGGTACCGTCTTGTAATCCTCCATCCAATGA \\
\hline oGQ488 & CCCCTGCAGATCAAGGCTGAAGGCTGCGAG \\
\hline oGQ489 & CCCTACCGGTACCGTCAAATAGTCAAATCCATCATCGTT \\
\hline oGQ490 & CCCAAGCTTTTGGAGGCGCAAACTTCTTGGAG \\
\hline oGQ491 & CCCTACCGGTACCGTCACGAATCGTAGCATTAAGCT \\
\hline oGQ492 & CCCAAGCTTTTGGAGGCGCAAACTTCTTGGAG \\
\hline oGQ493 & CCCTACCGGTACCCTTGAACTGTCGTCGGACATC \\
\hline oGQ494 & CCCAAGCTTAACATTCAACTTACGCTTTCC \\
\hline oGQ495 & CCCTACCGGTACCGTCACGTTGGTTTCGAGCATTTA \\
\hline oGQ496 & CCCAAGCTTCCACCGGAAAGCACTAAATTG \\
\hline oGQ497 & CCCTACCGGTACCGTGTTCAGATGCTCCGACAT \\
\hline oGQ498 & CCCCTGCAGCCCTTGTCATCGACTTCACAC \\
\hline oGQ499 & CCCTACCGGTACCGCTTCTCCCATTGTTGTCATTT \\
\hline oGQ556 & GTCGGCAAGAACTGCGTACTGTTCCGGTTCTCA \\
\hline oGQ557 & CAGTACGCAGTTCTTGCCGACTCCACTGTCTCC \\
\hline oGQ558 & ACAGCCGGGCTGGAGAGATTCAGAACTATCACA \\
\hline oGQ559 & GAATCTCTCCAGCCCGGCTGTGTCCCAAATTTG \\
\hline oGQ560 & GTTGGGAAAAACTGTCTAGTCAGAAAGTTCACA \\
\hline oGQ561 & GACTAGACAGTTTTTCCCAACGCCCGCATTTCC \\
\hline oGQ562 & ACGGCAGGTCTGGAGAGGTTTCGATCGATTACG \\
\hline
\end{tabular}




\begin{tabular}{|c|c|}
\hline oGQ563 & AAACCTCTCCAGACCTGCCGTATCCCAAATTTG \\
\hline oGQ564 & GTCGGAAAGAACTGTGTAGTTCAACGCTTCAGA \\
\hline oGQ565 & AACTACACAGTTCTTTCCGACTCCCATATCGCC \\
\hline oGQ566 & ACTGGAGGCCTGGAACGATTCCGAACGATTACT \\
\hline oGQ567 & GAATCGTTCCAGGCCTCCAGTATCCCAGATTTG \\
\hline oGQ568 & GTTGGTAAAAACTCACTTGTGCTTCGATTCGTT \\
\hline oGQ569 & CACAAGTGAGTTTTTACCAACACATCCTTCGCC \\
\hline oGQ570 & ACCGCTGGACTGGAGAAATACCACGCACTCGGT \\
\hline oGQ571 & GTATTTCTCCAGTCCAGCGGTATCCCAGATGTG \\
\hline oGQ572 & AGTGGAAAGAACTCAATTTGCCAGAGATTTGCA \\
\hline oGQ573 & GCAAATTGAGTTCTTTCCACTTGCTCCATCTCC \\
\hline oGQ574 & ATTGGAGGACTGAGTATTGCTGGTGAAATGATC \\
\hline oGQ575 & AGCAATACTCAGTCCTCCAATATCCCATACCTG \\
\hline oGQ576 & GTCGGAAAAAACTGTCTTTCATTCCGTTTTTGT \\
\hline oGQ577 & TGAAAGACAGTTTTTTCCGACGGCCGCGTTGCC \\
\hline oGQ578 & ACTGCTGGACTGGAAAGATATCGACAATCGATT \\
\hline oGQ579 & ATATCTTTCCAGTCCAGCAGTATCCCATAGCTG \\
\hline oGQ580 & GTAGGGAAGAACAGCTTAATGCTACGATTCGTG \\
\hline oGQ581 & CATTAAGCTGTTCTTCCCTACGCCACTTTCGCC \\
\hline oGQ582 & ACCGCCGGCCTGGAGAGGTTCCGTACCCTCACG \\
\hline oGQ583 & GAACCTCTCCAGGCCGGCGGTGTCCCAAATTGC \\
\hline oGQ590 & AGATCTCATATGGACAACGATGATGGATTT \\
\hline oGQ591 & CCCGAATTCTCAAGTGTACTGACAACATCG \\
\hline oGQ591 & CCCGAATTCTCAAGTGTACTGACAACATCG \\
\hline oGQ621 & CCCCTGCAGTCGGGATGATCTTCTATCTGG \\
\hline oGQ622 & CCCTACCGGTACCGTCGTCGCCGACTCTACTTCCATCTA \\
\hline oGQ623 & CCCCTGCAGGGGGAGTCATTTATCTCAAGTAAG \\
\hline oGQ624 & CCCTACCGGTACCTGATGATTGTAGCTCATTGAAGA \\
\hline oGQ625 & CCCACCTGCATGCAGCTTCGAAGATCTGTTGTGACTG \\
\hline oGQ626 & CCCTACCGGTACCGTGAACACATGATCTGATGACATTT \\
\hline oGQ627 & CCCAAGCTTTCGAAGATCTGTTGTGACTG \\
\hline oGQ628 & CCCTACCGGTACCGTGTGATCAGGGTACATTTCGGA \\
\hline oGQ640 & GGATCCTCCATGGAAGTAGAGTCGGCGACG \\
\hline oGQ641 & CTCGAGTCAAATACAGCACCAGCTCCT \\
\hline oGQ642 & GGATCCCATATGAGCTACAATCATCTTTTC \\
\hline oGQ643 & GGGAATTCAGACAAAGGCGGGTTCCGG \\
\hline oGQ648 & GGATCCCATATGCAAGTGCTCCGTCAACTA \\
\hline oGQ649 & GAATTCCTAGAGCATTGAACAACACTT \\
\hline oGQ673 & GTAGGCAAAAACTCTCTCGTATTGCGATTCGTC \\
\hline oGQ674 & TACGAGAGAGTTTTTGCCTACAGCACTTTCTCC \\
\hline oGQ675 & ACTGCAGGACTGGAAAGATATCATTCATTGGCA \\
\hline oGQ676 & ATATCTTTCCAGTCCTGCAGTATCCCAGATTTC \\
\hline oGQ677 & CCCAAGCTTGTTGATTTCCATTTGAATATGCGGCTAAAG \\
\hline oGQ678 & CCCTACCGGTACCCGTCCATAGTCGTCACCAATGAAGTTTGTTTC \\
\hline oGQ679 & CCCCTGCAGAAATTACTGAATGAACAACATCTTCGTCATATG \\
\hline oGQ680 & CCCTACCGGTACCCGTAGTTGACGGAGCACTTGCATTATCTTGT \\
\hline oGQ684 & CCCACCTGCATGCAGCTGTTTACAGGCTTTAGCTCCTCTTCTTCGAC \\
\hline oGQ685 & CCCTACCGGTACCGTCAATTTGAACAAATGATCGTAATCCCGAGTTCC \\
\hline oGQ730 & CCCAAGCTTGATATCTTTGAAATGTGTAAGATGGATTCG \\
\hline oGQ731 & CCCTACCGGTACCGTGTTTGGACCTCCAGGCCGGG \\
\hline oGQ732 & CCCAAGCTTGATATCTATTACGGGTGTCTAGGAGTAGC \\
\hline oGQ733 & CCCTACCGGTACCTCGTTATTTGTGAAATCAGCCATCTGTA \\
\hline oGQ734 & ATCAAGCTTGTAATCGCATATCAGGCCTC \\
\hline
\end{tabular}




\begin{tabular}{|c|c|}
\hline oGQ735 & CCCTACCGGTACCGGATTACCAAAGTCCGACATT \\
\hline oGQ736 & ATCCCGCCAGCGACGGAATGAGG \\
\hline oGQ737 & CCCTACCGGTACCGCCTTCTTTCTGGTTCCCGACATTT \\
\hline oGQ738 & CCCAAGCTTGATATCCCTTCTTTCAGCCTCAGCTTCAGC \\
\hline oGQ739 & CCCTACCGGTACCGCATACGGTCGGCGAGCCATTT \\
\hline oGQ740 & CCCCTGCAGGATATCATTGTGAAATATCGACTTTCTTGC \\
\hline oGQ741 & CCCTACCGGTACCGAGTCACGAGAGCCCATCTTTACTGC \\
\hline oGQ742 & CCCCTGCAGGATATCATATGTATATATGTTGCGAACTCAC \\
\hline oGQ743 & CCCTACCGGTACCGAGTAGTAGTATTCGTTGCCCATTT \\
\hline oGQ744 & CCCCTGCAGGATATCTCCAGAAAGCAACCCTGGAGAAG \\
\hline oGQ745 & CCCTACCGGTACCTGATAGTCGTAGTCACCCATCTTCCT \\
\hline oGQ815 & GTCTCCTGCTCCGAGAGCTAA \\
\hline oGQ819 & TGTAAATAAGACAATTTCGCCCGG \\
\hline oGQ851 & $\begin{array}{l}\text { GCTAGCGGCGGCCGCAGCGCTGGACCGGTAGGTATGACAACAATGGGAG } \\
\text { AAGACGAG }\end{array}$ \\
\hline oGQ852 & $\begin{array}{l}\text { GCTAGCGGCGGCCGCAGCGCTGGACCGGTAGGTATGGAGGATTACAAGT } \\
\text { ATCTATTC }\end{array}$ \\
\hline oGQ866 & CCCCTGCAGAGGGACACCCATTACATTCTGAC \\
\hline oGQ867 & CCCTACCGGTACCCTGTCACCAAGTAGCATAACCTTT \\
\hline oGQ869 & CCCTACCGGTACCCTTTGATATGGCATTGTTGTGAGC \\
\hline oGQ941 & CCCGGATCCGCGGTACCGGTACATATGCTTCGAAGGTTGAAAGAA \\
\hline oGQ942 & CCCCCCCTCGAGTCATTTCTGGGAATCAGTGAAGCACGT \\
\hline oGQ943 & CCCAAGCTTGTGACAATTCCATAGTTTCGGCAC \\
\hline oGQ944 & CCCTACCGGTACCGCGTTATTTGTGAGTGCTGCCATTT \\
\hline oGQ977 & CAAAATTCCGAAACGTTAAGTTGACCTCTATTCAAAAAACAA \\
\hline oGQ978 & AGCGCAAGGTAAGTTTAGCTTGAACTAATTCTGTTTAGAATG \\
\hline oGQ979 & CTTGATTTAATTTTAGAAAAATTACAGAAAAATGTCGGACTT \\
\hline oGQ980 & GTTGGAGTCTGCAAAAAAGTTGGGGTTAAATGAAGCTTTTTT \\
\hline oGQ995 & GTTGGAAAAAACTCTTTGATGAATCAATATGTA \\
\hline oGQ996 & CATCAAAGAGTTTTTTCCAACGCCCGAATCTCC \\
\hline oGQ997 & ACAGCCGGCCTGGAACGTTTCCAATCGCTCGGA \\
\hline oGQ998 & GAAACGTTCCAGGCCGGCTGTATCCCAGATCTG \\
\hline oGQ1078 & CTTCAGCAACGGCTTGTCTAA \\
\hline oGQ1127 & GGGGTACCGGTATCCATGGGCTCTCGTGACGATGAA \\
\hline oGQ1128 & CCCCGAATTCTCGAGTTATGGGATGCAACACTGCTT \\
\hline oGQ1129 & GGGGTACCGGTATCCATGGGCAACGAATACTACTAC \\
\hline oGQ1130 & CCCGAATTCTCGAGTTATGGAAAGCAACACTGGTT \\
\hline oGQ1131 & GTTGGAAAAAACTCATTCCTCTTCCGTTACTGT \\
\hline oGQ1132 & GAGGAATGAGTTTTTTCCAACTGATGAATTTCC \\
\hline oGQ1133 & ACCGCCGGACTGGAGAGGTACCGTACCATCACC \\
\hline oGQ1134 & GTACCTCTCCAGTCCGGCGGTATCCCAGATTTG \\
\hline oGQ1135 & GTTGGAAAAAACTGCATTCTGTACAGATTTTCG \\
\hline oGQ1136 & CAGAATGCAGTTTTTTCCAACTCCTGAGTCGCC \\
\hline oGQ1137 & ACAGCTGGACTGGAACGATTCCACACAATCACA \\
\hline oGQ1138 & GAATCGTTCCAGTCCAGCTGTGTCCCAGATTTG \\
\hline oGQ1139 & GTCGGAAAGAACAATCTCCTGTCTCGTTTC \\
\hline oGQ1140 & CAGGAGATTGTTCTTTCCGACGCCTGAGTCTCC \\
\hline oGQ1141 & ACTGCTGGACTGGAACGTTACCGTGCCATCACA \\
\hline oGQ1142 & GTAACGTTCCAGTCCAGCAGTATCCCAAATTTG \\
\hline oGQ1148 & GTCGGAAAAAACTCGTTTTTGCATCGTTACACG \\
\hline oGQ1149 & CAAAAACGAGTTTTTTCCGACTCCCGAATCGCC \\
\hline oGQ1150 & ACTGCCGGACTGGAGAGATTCCGTTCTCTAACA \\
\hline oGQ1151 & GAATCTCTCCAGTCCGGCAGTGTCCCATAACTG \\
\hline oGQ1152 & GTCGGAAAGAACAGCTTGTTGCTCCGTTTTGCA \\
\hline
\end{tabular}




\begin{tabular}{|c|c|}
\hline oGQ1153 & CAACAAGCTGTTCTTTCCGACACCCGAATCTCC \\
\hline oGQ1154 & ACTGCCGGGCTGGAGAGATTCCGTACAATTACA \\
\hline oGQ1155 & GAATCTCTCCAGCCCGGCAGTATCCCAAATCTG \\
\hline oGQ1156 & ACTGGAAAAAACTGCCTTCTGATTCGATACAAA \\
\hline oGQ1157 & CAGAAGGCAGTTTTTTCCAGTGCAGCTGTCACC \\
\hline oGQ1158 & ACCGCGGGACTGGAACGTTTTCGATCAGTGACC \\
\hline oGQ1159 & AAAACGTTCCAGTCCCGCGGTGTCCCAAATCTG \\
\hline oGQ1160 & GTTGGCAAAAACAGTCTTTTACGGTACTTCACA \\
\hline oGQ1161 & TAAAAGACTGTTTTTGCCAACTGTTGAATCCCC \\
\hline oGQ1162 & ACAGCAGGACTGGAAAAATTCAGATCAATTACA \\
\hline oGQ1163 & GAATTTTTCCAGTCCTGCTGTATCCCATAGTTG \\
\hline oGQ1164 & GTTGGAAAAAACTGTTTTCTACATAGATTTTGT \\
\hline oGQ1165 & TAGAAAACAGTTTTTTCCAACAGCCGAATCACC \\
\hline oGQ1166 & ACAGCAGGGCTGGAAAGATTCCGCTCAATCACA \\
\hline oGQ1167 & GAATCTTTCCAGCCCTGCTGTATCCCATAACTG \\
\hline oGQ1168 & TGCGGGAAAAACTGCATATTGCTGCGATTCGCT \\
\hline oGQ1169 & CAATATGCAGTTTTTCCCGCAATTGTGATCTCC \\
\hline oGQ1170 & ACCGCTGGACTGGAACGGTATAGGACTATATAC \\
\hline oGQ1171 & ATACCGTTCCAGTCCAGCGGTATCCCATAGTTC \\
\hline oGQ1172 & GTTGGAAAGAACTCAATTATCTATCGTCATCGA \\
\hline oGQ1173 & GATAATTGAGTTCTTTCCAACACCAGAATCTCC \\
\hline oGQ1174 & ACTGCTGGTCTGGAGAGGTTCCGATGCATGGTT \\
\hline oGQ1175 & GAACCTCTCCAGACCAGCAGTATCCCACACTTG \\
\hline oGQ1176 & GGGGTACCGGTATCCATGGCAGCACTCACAAATAAC \\
\hline oGQ1177 & CCCCGAATTCTCGAGTTAGCAACATTTCGAGTCGTA \\
\hline oGQ1178 & GTCGGTAAAAACTCTATTATTCGTCGATTTGTT \\
\hline oGQ1179 & AATAATAGAGTTTTTACCGACACCTGGATCACC \\
\hline oGQ1180 & ATTTCAGGCCTGGACCGATATGGGGTCATGACC \\
\hline oGQ1181 & ATATCGGTCCAGGCCTGAAATATCCCAGATTTG \\
\hline oGQ1185 & CTTCACACATCTCATGCCATC \\
\hline oGQ1186 & TCGGATGATGCCTTTAACACC \\
\hline oGQ1188 & CGTCAGTGGAATCAGGCATCT \\
\hline oGQ1193 & TGTTAATTATCTGCAGTTTGGAG \\
\hline oGQ1194 & AGCGTCTCGTGCAACGACATCTG \\
\hline oGQ1195 & CGAAATTTGAGTGAATTTGCCGGAAA \\
\hline oGQ1198 & CGAGAATTTCCTAGCATCCA \\
\hline oGQ1267 & GTTGGCAACAAGGCAGATATGG \\
\hline oGQ1268 & CCAGCCGTTATTCGTTCTCTC \\
\hline oGQ1271 & CCAACTACACACCAGCATCG \\
\hline oGQ1278 & TCGTAACTTCCTTCACTACGCTC \\
\hline oGQ1280 & CCATCCTCGACGAAACGCTC \\
\hline oGQ1283 & CTTGATTCCGTTTGCCTCATTG \\
\hline oGQ1288 & CTGATCGCATCCGGCTAGTT \\
\hline oGQ1290 & CGCTGGTATATGAATCTGAC \\
\hline oGQ1293 & CGATCCACCGACGTTCACAC \\
\hline oGQ1295 & GGGCACACTACTTTCAACACTC \\
\hline oGQ1297 & GTGTACTGACAACATCGTTGC \\
\hline oGQ1298 & GCAGCTCCGAATGTTGTGAAG \\
\hline oGQ1300 & GCTACACTTTCTGGTCGATGAG \\
\hline oGQ1302 & GATGGAGGAAATGCCAATGCG \\
\hline oGQ1305 & GTTGTCGAGGGCAGATGTTTC \\
\hline oGQ1307 & GTGACGAGAATTTCGAGCTG \\
\hline oGQ1310 & GATCGTTTATTGCAGGACGAG \\
\hline
\end{tabular}




\begin{tabular}{|c|c|}
\hline oGQ1314 & GATATGAGGACAGGGTGTGTGTTG \\
\hline oGQ1317 & CCTGAAATTACGTGGTGGCC \\
\hline oGQ1319 & TCCATCTTTCTGGAAATGCC \\
\hline oGQ1321 & TTGCAGTGACATGTTTCGGT \\
\hline oGQ1322 & CCAGTTGCCAGAATTGATTTCG \\
\hline oGQ1324 & CTGAAGTTTGACACGTTGTCC \\
\hline oGQ1327 & GATGCCTAACCAGAGAAGGTG \\
\hline oGQ1328 & CGATTCGCTTTCCTCAGCTCC \\
\hline oGQ1329 & CTCAACGTCTGTCCTCATTGTTC \\
\hline oGQ1331 & GTGGAAACTTGGGCTTTAGC \\
\hline oGQ1333 & GGAGCAGGGTATTTGGAGAA \\
\hline oGQ1334 & TAATCTTGCGAAGACAGCGGAG \\
\hline oGQ1336 & GGTGACACTGGATGAAGGAG \\
\hline oGQ1339 & CTGTCCTGCTGTATCCCATAG \\
\hline oGQ1343 & CCCATGGTACCGGTACATATGTCGAAACCCGAGGTGGAG \\
\hline oGQ1344 & CCCGAATTCTTAAGAACCACGACACTTTGAGAA \\
\hline oGQ1345 & GTTGGAAAAAACAGTTTTGTAATGCGGGTGATC \\
\hline oGQ1346 & TACAAAACTGTTTTTTCCAACAGCGGCGTCTCC \\
\hline oGQ1347 & ACCGCGGGACTGGAACGTTTCCGATCACTTTGT \\
\hline oGQ1348 & GAAACGTTCCAGTCCCGCGGTGTCCCATAGCTG \\
\hline oGQ1352 & CAGCGATCCGAGTATTTGACG \\
\hline oGQ1353 & GTCATCTGGTGTTCCTGCATTG \\
\hline oGQ1354 & CCACTGTGTTTAACGTGACAGC \\
\hline oGQ1357 & CATCTGACTTGTGCAAGCTCAC \\
\hline oGQ1358 & ACTGATGGGTAAGGAATCGAAGACG \\
\hline oGQ1359 & GATAAAACTTCGGAACATATGGGGC \\
\hline oGQ1399 & CAATACTTCGCTCCATACGGTG \\
\hline oGQ1475 & ATGACAGAACCGTCGGCTAGCGAA \\
\hline oGQ1476 & TCAATAAAACAAATTTTAATGCAC \\
\hline oGQ1492 & AGCTACTGCGTGGTATAATACTCTTCAGTA \\
\hline oGQ1493 & ACTTTGGCGAGAAGCTAGAGTCAAATG \\
\hline oGQ1494 & CGGTTGCCAATCAAGTTCAACTGAATGC \\
\hline oGQ1556 & AACGAACACAATTCCTAACTGAA \\
\hline oGQ1583 & GGATCCAGGGTACCGGTAATGGCGGGTATTCTTCGTCGACTT \\
\hline oGQ1584 & CCCCTCGAGTCAGCGGCCGCCACGGCGCCACCATTGAATAAG \\
\hline oGQ1590 & TTCCCGGGTTACCGGTTTCCATGGCAGCCACTGCAGCGCTACGT \\
\hline oGQ1601 & TTCCCGGGTTACCGGTTCATATGAGAACCCGCGCCCAAATTGGA \\
\hline oGQ1602 & GGGGCGGCCGCCCTCGAGCTAGTGGCTATCCGACAGTTGTACACC \\
\hline oGQ1688 & TCTACAGATGCCAAAGGTAAGTGAACA \\
\hline oGQ1689 & ACGAACTCGTCGAGCATTCATGCTATT \\
\hline oGQ1697 & GGGCTCGAGCTAGCGGCCGCCGTGGCTATCCGACAGTTGTACACC \\
\hline oGQ1700 & GACATTCCCGCTACCTACCCAGAGCTCGACTTT \\
\hline oGQ1701 & TGGGTAGGTAGCGGGAATGTCGCGTTGAATAAC \\
\hline oGQ1715 & ACCGGTAACAAATGGAAATCGGAACGAT \\
\hline oGQ1815 & CACGAAGACGCATACATTGAC \\
\hline oGQ2020 & TTTGGGTCCTGGAAAGAAAA \\
\hline oGQ2022 & TTCTAGCACACGGATGATGC \\
\hline oGQ2092 & CCCGGGTTACCGGTTTCCGAGGAAGACTTGTGGTCCGTG \\
\hline oGQ2093 & GGGCTCGAGTTAGCGATTTTCGGTACGAAGTCG \\
\hline oGQ2198 & CTCGAGCTAGGGACGCTTCTTGACTTCAAT \\
\hline
\end{tabular}




\subsection{Media}

\section{Luria-Bertani (LB) Medium}

$\begin{array}{ll}5 \mathrm{~g} / 1 & \text { Peptone (Roth) } \\ 2.5 \mathrm{~g} / 1 & \text { Yeast extract (Roth) } \\ 5 \mathrm{~g} / 1 & \text { NaCl (Roth) } \\ 7.5 \mathrm{~g} / 1 & \text { Agar (Roth), for plates } \\ 100 \mu \mathrm{g} / \mathrm{ml} & \text { Ampicillin (Roth) } \\ 15 \mu \mathrm{g} / \mathrm{ml} & \text { Kanamycin (Roth) }\end{array}$

\section{Nematode Growth Medium (NGM)}

\begin{tabular}{|c|c|}
\hline $16 \mathrm{~g} / 1$ & Agar \\
\hline $3 \mathrm{~g} / 1$ & Peptone \\
\hline $3 \mathrm{~g} / 1$ & $\mathrm{NaCl}$ \\
\hline $25 \mathrm{ml} / 1$ & $1 \mathrm{M} \mathrm{KPO}_{4}$ Solution (Roth) \\
\hline $1 \mathrm{ml} / 1$ & $1 \mathrm{M} \mathrm{MgSO}_{4}$ Solution (Roth) \\
\hline $1 \mathrm{ml} / 1$ & $1 \mathrm{M} \mathrm{CaCl}_{2}$ Solution (Roth) \\
\hline $1 \mathrm{ml} / 1$ & 10 mg/ml Nystatin (Sigma) \\
\hline $1 \mathrm{ml} / 1$ & $5 \mathrm{mg} / \mathrm{ml}$ Cholesterol (Roth) \\
\hline
\end{tabular}

\section{Complex (YAPD) yeast media}

$\begin{array}{ll}10 \mathrm{~g} / 1 & \text { Yeast extract } \\ 20 \mathrm{~g} / 1 & \text { Peptone } \\ 20 \mathrm{~g} / 1 & \text { Glucose (Roth) } \\ 1 \mathrm{~g} / 1 & \text { Adenine Sulfate (Roth) } \\ 20 \mathrm{~g} / 1 & \text { Select agar (Invitrogen) }\end{array}$

\section{Yeast minimal growth medium}
$6.8 \mathrm{~g} / 1$
$1.0 \mathrm{~g} / 1$
$20 \mathrm{~g} / 1$
Yeast nitrogen base (Invitrogen)
16 amino acid mix (-leu. -trp, -his, -ura)
Select agar, for plates
pH 6-7

After autoclaving:

$10 \mathrm{ml} / 1$

amino acid solution (+his $480 \mathrm{mg} / 200 \mathrm{ml}$ )

(+ura $480 \mathrm{mg} / 200 \mathrm{ml}$ )

$40 \mathrm{ml} / 1$

$50 \%$ Glucose 


\subsection{Buffers and solutions}

Worm lysis/PCR buffer (1x)
$100 \mathrm{mM}$
Tris $\mathrm{pH} 8.3$
$500 \mathrm{mM}$
$\mathrm{KCl}$ (Roth)
$20 \mathrm{mM}$
$\mathrm{MgCl}_{2}$ (Roth)
$20 \mathrm{mg} / \mathrm{ml}$
Proteinase K added fresh prior to lysis (Roth)

\section{Worm lysis/PCR buffer (10x)}

$\begin{array}{ll}1 \mathrm{M} & \text { Tris pH } 8.3 \\ 5 \mathrm{M} & \mathrm{KCl} \\ 200 \mathrm{mM} & \mathrm{MgCl}_{2}\end{array}$

TE buffer
$10 \mathrm{mM}$
$1 \mathrm{mM}$
Tris-HCl pH 8.0
EDTA (Roth)

TBE buffer (10x)
$108 \mathrm{~g} / 1$
Tris $\mathrm{NaOH}$
$55 \mathrm{~g} / 1$
Boric acid (Roth)
$9.3 \mathrm{~g} / 1$
EDTA (pH 8.0)

\section{M9 buffer}

$\begin{array}{ll}3 \mathrm{~g} / 1 & \mathrm{KH}_{2} \mathrm{PO}_{4} \text { (Roth) } \\ 6 \mathrm{~g} / 1 & \mathrm{Na}_{2} \mathrm{HPO}_{4} \text { (Roth) } \\ 5 \mathrm{~g} / 1 & \mathrm{NaCl} \text { (Roth) } \\ 1 \mathrm{ml} & 1 \mathrm{M} \mathrm{MgSO} \text { (Roth) }\end{array}$

GST cleavage buffer

$\begin{array}{ll}50 \mathrm{mM} & \text { Tris- } \mathrm{HCl} \mathrm{pH} 7.0-7.3 \text { (Roth) } \\ 150 \mathrm{mM} & \text { NaCl } \\ 1 \mathrm{mM} & \text { EDTA } \\ 2 \mathrm{mM} & \text { DTT (Roth) } \\ 100 \mu \mathrm{M} & \text { Pefabloc protease inhibitor cocktail (Roche) }\end{array}$

GST elution buffer

$\begin{array}{ll}50 \mathrm{mM} & \text { Tris- } \mathrm{HCl} \mathrm{pH} 8.0 \\ 150 \mathrm{mM} & \mathrm{NaCl} \\ 20 \mathrm{mM} & \text { reduced glutathione (Roth) }\end{array}$


PBS buffer (1x)

$\begin{array}{ll}8 \mathrm{~g} / 1 & \mathrm{NaCl} \\ 0.2 \mathrm{~g} / 1 & \mathrm{KCl} \\ 1.44 \mathrm{~g} / 1 & \mathrm{Na}_{2} \mathrm{HPO}_{4} \\ 0.24 \mathrm{~g} / 1 & \mathrm{KH}_{2} \mathrm{PO}_{4}\end{array}$

Yeast transformation 1-step buffer

$\begin{array}{ll}0.2 \mathrm{M} & \text { Lithium Acetate }(0.4 \mathrm{~g})(\text { Roth }) \\ 40 \% & \text { PEG } 4000(12-15 \mathrm{~g})(\text { Sigma }) \\ 100 \mathrm{mM} & \text { DTT }(0.46 \mathrm{~g})\end{array}$

Fill up to $30 \mathrm{ml}$ with $\mathrm{ddH}_{2} \mathrm{O}$ and filtrate

10\% APS solution

$\begin{array}{ll}1 \mathrm{~g} & \text { APS (Roth) } \\ 10 \mathrm{ml} & \mathrm{ddH}_{2} \mathrm{O}\end{array}$

SDS running buffer (10x)

$\begin{array}{ll}30.3 \mathrm{~g} / 1 & \text { Tris } \\ 144 \mathrm{~g} / 1 & \text { Glycine (Roth) } \\ 10 \mathrm{~g} / 1 & \text { SDS (Roth) } \\ & \text { pH } 8.3\end{array}$

2x SDS sample buffer

$\begin{array}{ll}121.1 \mathrm{~g} / 1 & \text { Tris pH } 6.8 \\ 20 \% & \text { Glycerol (v/v) } \\ 100 \mathrm{~g} / 1 & \text { SDS } \\ 0.002 \% & \text { Bromophenol blue (w/v) } \\ 200 \mathrm{ml} & \text { 2-Mercaptoethanol (Sigma Aldrich) }\end{array}$

5\% Stacking gel $-5 \mathrm{ml}$

$\begin{array}{ll}0.83 \mathrm{ml} & 30 \% \text { Acrylamide/Bis (Roth) } \\ 3.4 \mathrm{ml} & \mathrm{ddH}_{2} \mathrm{O} \\ 0.63 \mathrm{ml} & 1.0 \mathrm{M} \text { Tris, } \mathrm{pH} 6.8 \\ 50 \mu \mathrm{l} & 10 \% \text { SDS } \\ 50 \mu \mathrm{l} & 10 \% \text { APS (Roth) } \\ 5 \mu \mathrm{l} & \text { TEMED (Roth) }\end{array}$


$10 \%$ Resolving Gel - 15ml

$\begin{array}{ll}5 \mathrm{ml} & 30 \% \text { Acrylamide/Bis } \\ 5.9 \mathrm{ml} & \mathrm{ddH}_{2} \mathrm{O} \\ 3.8 \mathrm{ml} & 1.5 \mathrm{M} \text { Tris, } \mathrm{pH} 8.8 \\ 150 \mu \mathrm{l} & 10 \% \text { SDS } \\ 150 \mu \mathrm{l} & 10 \% \text { APS } \\ 6 \mu \mathrm{l} & \text { TEMED }\end{array}$

\section{Coomassie Blue Stain}
$0.25 \%$
$50 \%$
Coomassie Brilliant Blue R250 (BioRad)
$10 \%$
Methanol (Roth)
Acetic acid (Roth)

\section{Coomassie destainer}
$5 \%$
Methanol (Roth)
$7.5 \%$
Acetic acid (Roth)

\subsection{Gene structures of $t b c-4$ and $r a b n-5$ deletion alleles}

Since the $t b c-4$ and rabn-5 alleles used in this study were not published before, they are depicted below. Gene structures for all the rab mutants used in this study are depicted in Chapter 1: Results (IV).

\section{tbc-4}

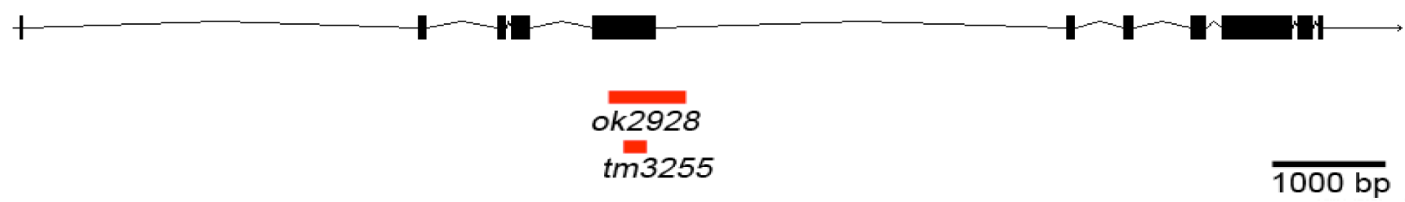

rabn-5

ok1555

Figure XII.1. Gene structures of $t b c-4$ and rabn-5. Two alleles of $t b c-4: t b c-4$ (ok2928), $t b c$ 4(tm3255) and one allele of rabn-5: rabn-5 (ok1555) were used in this study. The red line depicts the region that is deleted for the corresponding mutant alleles. 


\subsection{Multiple sequence alignment of TBC-4}

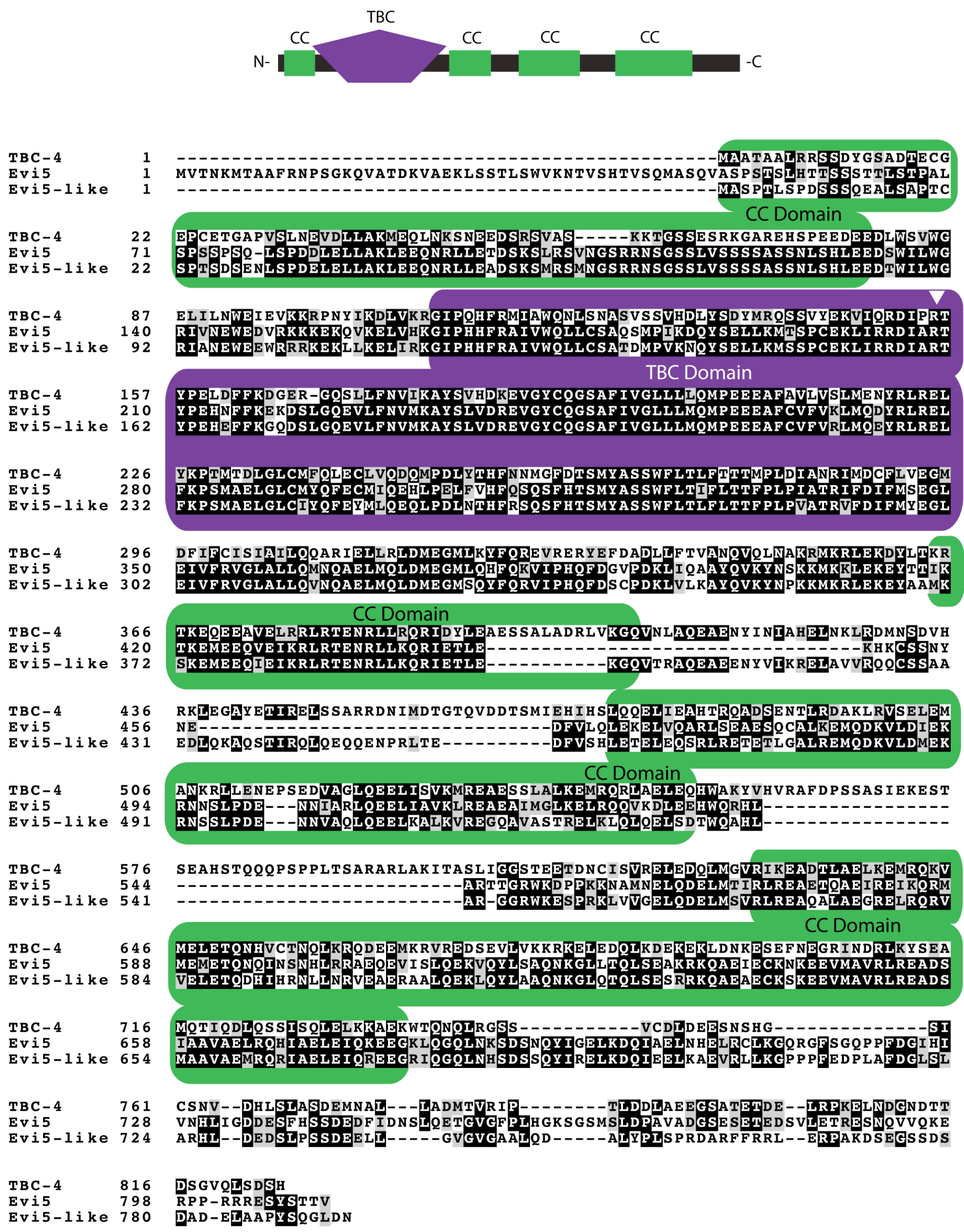

Figure XII.2. Multiple sequence alignment of TBC-4. The protein sequence of TBC-4 is shown aligned together with the closest human orthologues: Evi5 and Evi5-like. TBC-4 contains one TBC domain (shown in purple) and four coiled coil (CC) domains (shown in green). A white triangle depicts the catalytic arginine finger R155. Source: Multiple sequence alignment was done using the MUSCLE tool and the output was generated using BOXSHADE. 


\section{Curriculum Vitae}

NiKHIL SASIDHARAN

European Neuroscience Institute

Grisebachstrasse 5

37077 Göttingen, Germany

++4917627073539

nsasidh@gwdg.de

Nationality: Indian

Date of Birth: $\quad$ 14/10/1985

Place of Birth: Attingal, India

\section{EDUCATION}

10/2007 to date

09/2006-09/2007

08/2003-06/2006

$08 / 1999-05 / 2003$
$01 / 2007-03 / 2007$

$02 / 2006-06 / 2006$

\section{European Neuroscience Institute, Göttingen, Germany}

Pursuing PhD degree in Neurosciences, expected to finish in April 2011

Max Planck Research School, Göttingen, Germany

Completed preparatory year of coursework in Neurosciences prior to entering $\mathrm{PhD}$

Earned final grade A in M.Sc Examination

Jacobs University Bremen, Bremen, Germany

Earned a B.Sc. degree in Biochemistry and Cell Biology

Graduated in the top 5\% of class

Kodaikanal International School, Kodaikanal, India

Successfully completed high school education

Earned International Baccalaureate diploma in Mathematics, Physics and Chemistry

\section{PRACTICAL EXPERIENCE}

$10 / 2007$ to date

European Neuroscience Institute, Göttingen, Germany

$\mathrm{PhD}$ project in the laboratory of Dr. Stefan Eimer

Studying the role of Rab GTPases in neuronal membrane trafficking in C.elegans

Methods: Spinning-disk microscopy, transgenesis, assaying worm behaviors, molecular cloning, protein expression and purification, in vitro GTPase assays, high-throughput yeast-2-hybrid screening, high-throughput functional RNAi screening.

$03 / 2007-05 / 2007$

Max Planck Institute for Experimental Medicine, Göttingen, Germany

Laboratory Rotation in the neurobiology laboratory of Prof. Dr. Klaus Nave

Studied the role of Neuregulin1 in CNS myelination of young mice

Methods: Cre-Lox recombinant techniques, immunohistochemistry

Institute for Physiology, University of Goettingen, Göttingen, Germany

Laboratory Rotation in the neurophysiology laboratory of Dr. Weiqi Zhang

The effect of erythropoietin (EPO) on Pre-Boetzinger complex in mouse brain-stem Methods: Whole-cell patch clamp of brain-stem slices

Jacobs University Bremen, Bremen, Germany

B.Sc thesis project in the biochemistry laboratory of Prof. Dr. Albert Jeltsch

Studied the molecular enzymology of DNA Methyltransferase I (DNMT 1) in vivo using novel imaging assay 
$10 / 2005-02 / 2006$

$03 / 2007-04 / 2007$

$06 / 2006-07 / 2006$

$09 / 2001-05 / 2003$

$07 / 2009-08 / 2009$
Methods: Molecular cloning, Realtime PCR, Western Blotting, Fluorescence microscopy Jacobs University Bremen, Bremen, Germany

Teaching Assistant for the "Advanced Biochemistry and Cell Biology I Lab Course" Supervision of experiments, assistance in tutorials and grading of quizzes

Methods: SDS-PAGE, Agarose Gel Electrophoresis, Western Blotting, PCR

\section{EXTRACURRICULAR ACTIVITIES}

Roche Continents, Salzburg, Austria

Youth, Arts and Science program

Participated in workshops for team-building and creativity in arts and science

Max Planck Institute for Experimental Medicine, Göttingen, Germany

Organizing member of the Neurizons 2009, conference in the Neurosciences

Involved in general organization, website and booklet (http://www.neurizons.uni-goettingen.de/)

Max Planck Institute for Experimental Medicine, Göttingen, Germany

Indian Culture Night

Organizer of cultural events about India

Berlitz AG, Berlin, Germany

Kids and Teens - English Camp

English teacher for kids age group 8-12

Kodaikanal International School, Kodaikanal, India

Social aid project

Participated in providing aid and assisted in building infrastructures for target village

Malayandipuram, India

\section{SKILLS \& INTERESTS}

Languages

English: Fluent in written and spoken

German: Working knowledge

French: Advanced in written and spoken
PC Knowledge

Programming

Merits/Awards
Microsoft Office, Adobe illustrator and photoshop, ImageJ

HTML, and basic knowledge of pascal

GGNB excellence fellowship for PhD studies, Göttingen, Germany

Max Planck excellence stipend for PhD studies, Göttingen, Germany

Merit-based scholarship for undergraduate studies at the Jacobs University Bremen,

Germany

Hobbies

Learning to play guitar, hiking, squash and reading books of biology and history 


\section{LIST OF PUBLICATIONS}

Luo L., Hannemann M., Koenig S., Hegermann J., Ailion M., Cho M.-K., Sasidharan N., Zweckstetter M., Rensing S.A. and Eimer S. The C. elegans GARP complex contains the conserved Vps51 subunit and is required to maintain lysosomal morphology. In revision at $\mathrm{MBoC}$.

Butkevich E., Hegermann J., Sasidharan N., Lecaudey V., Eimer S.* and Klopfenstein D.R.*. The Kinesin-1 is required for dendritic cargo transport and microtubule integrity in Caenorhabditis elegans neurons. * Corresponding authors. In revision at J. Neurosci. 